\title{
6th European Federation for Primatology Meeting
}

\author{
XXII Italian Association of Primatology Congress \\ Rome, Italy, August 25-28, 2015 \\ Editors: Elisabetta Visalberghi, Monica Carosi, Gloria Sabbatini, Rome, Italy
}

\section{Ecology and Habitat Use of the Endangered Primate Macaca maura (H.R. Schinz, 1825), Endemic to the Karst Forests of Sulawesi Island, Indonesia}

Alessandro Albani ${ }^{a}$, Ngakan Putu Oka ${ }^{b}$, Lavinia Germania ${ }^{a}$ Monica Carosia

aUniversità degli Studi Roma Tre, Rome, Italy; bUniversitas Hasanuddin, Makassar, Indonesia

E-Mail: ale.albani@gmail.com

The Sulawesi endemic species Macaca maura has been listed in the IUCN Red List as Endangered (A2cd) since 1996, mainly due to habitat disturbance and fragmentation. Nowadays, residual populations have increasingly been relegated to the karst areas of the island's Southern District. The main goal of this project is to point out preferences and strategies adopted by the studied group $\left(\mathrm{N}_{\text {individuals }}=31\right)$ in the use of this rare habitat, in order to contribute to the understanding of functional aspects of the spatial niche of the species. Hutcheson's diversity t-test on 34 vegetation plots yielded significant differences between the 'ground forest' and the 'karst tower forest' $(t=-6.31 ; p<0.05)$. The $t$ test showed significant differences also in terms of tree abundance $(\mathrm{t}=4.76 ; \mathrm{p}<0.05)$, average tree $\mathrm{DBH}(\mathrm{t}=-2.96 ; \mathrm{p}<0.05)$ and average canopy closure $(\mathrm{t}=$ $-4.61 ; \mathrm{p}<0.05)$, suggesting that the two forests could perhaps be considered as distinct microhabitats. Preliminary analyses on habitat use revealed preferential use of 'ground forest' by all group members during selected budget activities (locomotion; feeding and foraging; social behaviour; resting). On the other hand, no differences were found in selective use of the two habitats between sex classes ( 8 adult females, 4 adult males) or age classes ( 6 young adults, 6 old adults). As a multidisciplinary study design, the group's feeding ecology and health status will also be analysed for possible ecological correlates to habitat-use. In fact, a comparison between food selection and nutritional composition of preferred foods is currently being performed, in addition to enteric parasite analyses on stool samples (collected in accordance with Directive 2010/63/EU). Project results will help to understand whether the macaques' dispersed presence in the area of karst formations, which is hardly accessible by humans, is an ideal condition for the survival of the species.

\footnotetext{
KARGER 125 今 $\odot 2015$ S. Karger AG, Basel 


\section{Quantifying Fission-Fusion Dynamics: A Case Study of Olive Baboons}

Nienke Alberts ${ }^{a}$, Stuart Semple ${ }^{b}$, Julia Lehmann ${ }^{b}$

aUniversity College London, and buniversity of Roehampton, London, UK

E-Mail: nienke.alberts@ucl.ac.uk

Primate social groups have traditionally been described according to their social cohesion, with species classified as having either a cohesive or a fluid social system. In recent years this modal classification has come under scrutiny. It has been suggested that primate groups should instead be characterised by their relative degree of fission-fusion dynamics, which refers to the variation in spatial cohesion and individual group membership over time. Whilst the concept of fission-fusion dynamics has become generally accepted, its central framework has not been implemented. Rather than quantifying the degree of fission-fusion dynamics, this measure has been used anecdotally, with study groups being described as having either a low or a high degree of fission-fusion dynamics. Quantification of fission-fusion dynamics is crucial for making comparisons across populations and species. Here, we propose and explore two complementary methods for quantifying fission-fusion dynamics, using olive baboons (Papio anubis) as a case study. We collected data on party size, party composition, spatial cohesion, and inter-individual distances in two troops (Gamgam \& Kwano) of wild olive baboons in Gashaka-Gumti National Park, Nigeria, over a 12-month period. First, fission-fusion dynamics where quantified by the coefficient of variation for the measurements in party size, the rate of party composition change, and inter-individual distances of focal animals. Second, we fitted single and mixed distributions to the inter-individual distances as a measure of the degree of fragmentation. We found that the two methods gave us similar results, and the troops differed in their degree of fission-fusion dynamics. The Gamgam troop rarely fragmented and parties were often large. The Kwano troop, by contrast, frequently split into small parties that were relatively stable over time. These results indicate that fission-fusion dynamics can differ between troops within a single population.

\section{Play as an Indicator of Animal Welfare in a Captive Colony of Cebus apella}

Olimpia Algora Ortega ${ }^{a}$, Elisa Demuru $^{a}$, Elisabetta Palagi ${ }^{a, b}$

aNatural History Museum, University of Pisa, Calci, and b Institute of Cognitive Sciences and Technologies, Unit of Cognitive Primatology and Primate Center (CNR), Rome, Italy E-Mail: olimpiaalgora@gmail.com

Play behaviour is a potential indicator of animal welfare. The self-rewarding nature of play and its tendency to spread within the group make this behaviour an excellent mean to estimate the current (and potentially future) wellbeing of a single individual, as well as of its entire social group. Visual communication has a fundamental role in triggering and regulating a playful session by modulating the patterns through inhibitory and reinforcement mechanisms. This research evaluates the individual and collective welfare of a captive colony of 20 Cebus apella through the description of playful patterns and facial expressions. Facial expressions were then subdivided into two categories: playful facial expressions (i.e. those occurring exclusively during play) and all the others (i.e. those occurring also in other contexts). As occurs in numerous mammalian species, immature subjects showed a higher frequency of play behaviour than adults. Among adults, males played more than females. Although playful facial expressions were displayed at similar frequencies in all age/sex classes, the other facial expressions were exhibited more frequently by females and especially when the playmate could visually perceive the signal. Within the studied colony, only three subjects were never observed playing. These animals were socially isolated and characterized by anomalous behaviours. Taken together, all these findings strongly suggest that play behaviour should be considered as a highly reliable and accurate indicator of captive animal welfare. 


\section{The Effect of Aging on Exploration and Social Interest in Barbary Macaques, Macaca sylvanus}

Laura Almeling, Kurt Hammerschmidt, Julia Fischer

Cognitive Ethology Laboratory, German Primate Centre, Göttingen, Germany

E-Mail: LAlmeling@dpz.eu

Social bonds have been shown to promote well-being and life expectancy. With increasing age, however, overall social activity declines. For humans, various explanatory models have been put forward to explain this decline. One key question is whether older individuals generally loose interest, including in the physical domain (disengagement theory). In contrast, interest might not change (activity theory). Alternatively, ageing individuals may become more selective with age (socio-emotional selectivity theory), showing a decreased interest in exploring new things and superficial contacts, but focussing on important social partners. We tested this question in Barbary macaques ( $\mathrm{Ma}$ caca sylvanus) ranging in the park 'La Forêt des Singes' in Rocamadour, France. In order to determine their interest in the physical environment, we presented subjects of different ages with three types of novel objects, one containing a food reward. In the social domain, we used female subjects' behavioural response to playbacks of recruitment screams as a measure of social interest. We played subjects of different ages recruitment screams of two categories of social partners: 'best-friend' (i.e. the female partner with whom most affiliative interactions had been exchanged), 'non-friend' (i.e. a female group member with which no affiliative interactions had been exchanged). Age had a negative effect on exploration of all novel objects. Females attended more strongly to recruitment calls of friends than to those of non-friends, but there was no effect of age. Results are discussed in the framework of human social aging theories. The study complied with the European Directive 2010/63/EU.

\section{Sugarcane as Staple Fallback Food for Blond Capuchin Monkeys, Sapajus flavius}

Poliana Gabriele Alves de Souza Lins, Renata Gonçalves Ferreira

Psychobiology Graduation Program, Federal University of Rio Grande do Norte, Natal, Brazil E-Mail: polianagasl@gmail.com

The socio-ecological consequences of non-preferred food consumption by primates depend on the type of fallback foods (FBF) used. Reliance on staple FBF drives food processing adaptations (digestive and cognitive) and allows more stable group formation, while foraging on filler FBF drives harvesting adaptations. Capuchin monkeys are known for their dietary flexibility and manipulative destructive foraging habits, which suggest reliance on staple FBF, such as consumption of nuts, cracked using stones, in dry-caatinga areas. In this work we analysed whether sugarcane constitutes preferred, staple or filler fallback food for a group of about 80 blond capuchin monkeys (Sapajus flavius) that inhabit a 240 ha fragment of Atlantic forest, surrounded by sugarcane plantation. This research complied with the International Primatological Society (IPS) Guidelines for the Use of Nonhuman Primates in Research, since we only observed the animals and did not used any invasive method in the research. We compared the consumption of different food items with their monthly availability in the area. We found that fruit feeding time correlated with fruit productivity, indicating preference for fruits. Available all year round and uniformly distributed, sugarcane was a regular item in the diet. However, it never comprised $0 \%$ of the diet, and reached over $70 \%$ of the food consumed during the months of low fruit availability, characterizing its use as a staple FBF by this group. In accordance with model predictions, the group remained stable and immatures were observed all year round. We suggest that the juvenile stage is a critical survival period for these capuchins, as the monkeys need to develop the ability to process sugarcane for consumption. Moreover, these data show the importance of a sugarcane landscape for the survival of this critically endangered capuchin species in fragmented habitats in northeast Brazil.

6th European Federation for Primatology Meeting
Folia Primatol 2015;86:235-386 DOI: $10.1159 / 000435825$ 


\title{
Feeding Competition in a Semi-Free-Ranging Group of Blond Capuchin Monkeys, Sapajus flavius
}

\author{
Poliana Gabriele Alves de Souza Lins, Renata Gonçalves Ferreira \\ Psychobiology Graduation Program, Federal University of Rio Grande do Norte, Natal, Brazil \\ E-Mail: polianagasl@gmail.com
}

Contest competition will increase when individuals make use of clumped or monopolizable resources, while scramble competition increases when individuals make use of non-monopolizable or dispersed food items. We observed the intensity of contest and scramble competition in a group of about 80 blond capuchin monkeys (Sapajus flavius) that inhabit a 240 ha fragment of Atlantic forest, surrounded by sugarcane plantation. Direct competition was measured by the number of actual conflicts observed. Indirect competition was measured by the area formed by all group members. This was done using the Minimum Convex Polygon (individual positions recorded during group scans) and by the number of neighbours at a $5 \mathrm{~m}$ distance from focal animals. Direct competition occurred at a rate of 0.38 events per hour, three to six times the rate described for free-ranging capuchins (Sapajus spp.). However, although fruits are preferential food items, direct competition rate did not correlate to fruit productivity in the area. The mean MCP area was $450 \pm 476 \mathrm{~m}^{2}$, or $25 \pm 27 \mathrm{~m}^{2}$ if we divide MCP by number of individuals included in each scan. The mean number of neighbours up to $5 \mathrm{~m}$ was $1.17 \pm 1.28$. The MCP/ind positively correlated with rainfall, and the number of neighbours comprising females carrying infants was greater in periods of increased fruit productivity. Indirect competition was not influenced by fruit or insect availability. The results indicate that, although blonde capuchins in this fragment make use of sugarcane as a staple fallback food, animals are facing a high intensity of direct competition which lasts throughout the year, suggesting that the area does not offer sufficient food for such a large group in either period of the year. In addition, our data indicate that females carrying infants are suffering increased indirect competition when fruit productivity is low. This research complied with the International Primatological Society Guidelines for the Use of Nonhuman Primates in Research.

\section{When the Social Context Changes: Social Inhibition and Fission-Fusion Dynamics}

Federica Amici ${ }^{a, b}$, Josep Calla, $c$, Sarah Brosnan ${ }^{d}$, Julia Watzek ${ }^{d}$, Filippo Aurelie, $f$

aDepartment of Comparative and Developmental Psychology, Max Planck Institute for Evolutionary Anthropology, Leipzig, Germany; ' Institute of Psychology, University of Bern, Bern, Switzerland; 'School of Psychology and Neuroscience, University of St Andrews,

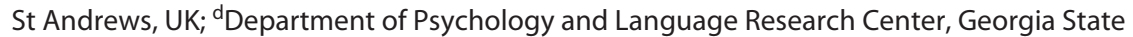
University, Atlanta, Ga., USA; 'Instituto de Neuroetologia, Universidad Veracruzana, Xalapa, Veracruz, Mexico; 'Research Centre in Evolutionary Anthropology and Palaeoecology, Liverpool John Moores University, Liverpool, UK

E-Mail: amici@eva.mpg.de

High levels of fission-fusion dynamics (FFD) may constitute a form of social complexity and have been linked to the enhancement of specific cognitive skills. Species with high levels of FFD, for example, experience frequent changes in subgroup size and composition. If the appropriate response to a situation depends on the social context, and the social context frequently varies, a high inhibitory tendency might be selectively advantageous to suppress prepotent but inappropriate responses and better assess the situation before acting. In this study, we tested social inhibition (i.e. the ability to suppress a prepotent response depending on the social context) in 6 primate species differing in their levels of FFD: chimpanzees, bonobos, orangutans and spider mon- 
keys, which are characterized by relatively high levels of FFD, and gorillas and capuchin monkeys, which are characterized by lower levels of FFD. Subjects could choose between a larger and a smaller food reward. Smaller food rewards were always accessible to the subject, but accessibility to the larger food rewards depended on the social context, i.e. on the location of a partner. When the partners' position allowed them to receive the larger food reward, subjects had to inhibit its selection to avoid obtaining no food at all during the trial. Our results suggest a link between the levels of FFD and social inhibition tendencies across species, especially during the first trials, although experience with the set-up allowed all species to generally increase performance in the task over trials. Our study contributes to the understanding of the importance of using a socioecologically valid framework to compare specific cognitive skills across species. Our research complied with the European Directive 2010/63/EU Guidelines for the Use of Nonhuman Primates in Research.

\title{
Contextual Variation of the Vocalizations of Propithecus diadema in the Wild
}

\author{
Alessio Anania ${ }^{a}$, Francesco Costa ${ }^{a}$, Giovanna Bonadonna ${ }^{a}$, Olivier Friard ${ }^{a}$, \\ Jonah H. Ratsimbazafy ${ }^{b}$, Marco Gamba $^{a}$, Cristina Giacoma ${ }^{a}$ \\ ${ }^{a}$ Department of Life Science and Systems Biology, University of Turin, Torino, Italy; ${ }^{b}$ GERP, \\ Group d'étude et de recherche sur les primates de Madagascar, Antananarivo, Madagascar \\ E-Mail: alessio.anania87@gmail.com
}

Caller identity, sex, age, context, emotion and environmental factors can all contribute to the acoustic variation of primates calls. We studied the acoustic variation of different vocal types and whether within-vocal type variation occurred in different contexts. We investigated three habituated groups of diademed sifaka (Propithecus diadema) in the wild, in the Maromizaha rainforest, eastern Madagascar, between April and August 2014. The vocal communication of the diademed sifaka is scarcely known, and its vocal repertoire has never been studied with the support of quantitative analysis. In the present work, we focused on alarm calls and group coordination calls, used during group movement and group cohesion. We found that the loud Zzuss call, which is often followed by a tongue click, is used during vigilance, group coordination, intergroup encounter, aerial and terrestrial disturbance. The acoustic structure of the Zzuss call changed according to the context of emission (Mantel test, $\mathrm{p}<0.001$ ). We also found a contextual variation for long-range Hoo calls when they were uttered for cohesion or lost contact (Mantel test, $\mathrm{p}<0.001$ ). The most common close-range call, the Hum, was frequently recorded during vigilance, but also during group movement and occasionally when the lemurs were feeding or allogrooming. The acoustic structure of the Hum did not significantly vary among different contexts (Mantel test, $\mathrm{p}=0.783$ ). We recorded the MMM call during aerial disturbance, vigilance and occasionally during cohesion, and we found a contextual variation (Mantel test, $p=0.007$ ). These results suggest a contextual variation for at least three vocal types (Zzuss, Hoo and MMM) whereas the most common close-range call, the Hum, is contextually invariant. This study complied with the International Primatological Society Guidelines for the Use of Nonhuman Primates in Research and a Research Permit was issued by the Malagasy Ministry of Environment and Forest. 


\title{
Weight versus Time Estimates: Methods to Estimate the Energy and Protein Intake in Black Howler Monkeys, Alouatta pigra
}

\author{
John F. Aristizabala, ${ }^{a}$, Jessica M. Rothman ${ }^{c}$, Luis M. García-Fería ${ }^{b}$, Juan Carlos Serio-Silva ${ }^{b}$, \\ Nicoletta Righini ${ }^{d, e}$

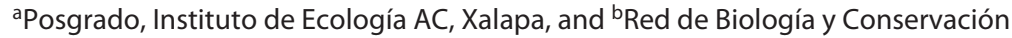 \\ de Vertebrados, Instituto de Ecología AC, Xalapa, Veracruz, México; ${ }^{\circ}$ Department of \\ Anthropology, Hunter College of the City University of New York, New York, N.Y., USA; \\ ${ }^{d}$ Red de Manejo Biorracional de Plagas y Vectores, Instituto de Ecología AC, \\ Xalapa, Veracruz, México; 'Department of Anthropology, University of Illinois at \\ Urbana-Champaign, Urbana, III., USA \\ E-Mail: john.aristizabal@posgrado.inecol.edu.mx
}

Two methods are commonly used to measure nutrient and energy intake in wild primates: time estimates (i.e., the time spent feeding on a specific plant part), and weight-based estimates (i.e., the ingested amount of a specific plant part). From previous research, it is known that howler monkeys (Alouatta spp.) eat fruit when it is available, but consume leaves almost daily. However, the majority of these studies are based on feeding time, which can hide more useful aspects for the understanding of the species' feeding behaviour and nutritional ecology. Here we present data on the diet of two groups of black howler monkeys (Alouatta pigra) living in a fragmented habitat in Balancán, Mexico to determine the reliability of these two intake estimates. We conducted behavioural observations ( $\mathrm{n}=658 \mathrm{~h}$ and $\mathrm{n}=46$ full day focal follows) from August 2012 to March 2013. For each feeding bout we determined feeding time ( $\mathrm{FT}=17 \%$ of total observation time), and amount of food ingested by counting the number of food items ingested by the focal animal. Time-based estimates revealed that howler monkeys had a predominantly folivorous diet, confirming previous reports. However, considering weight-based estimates, the diet was fruit-and-leaf based. According to the amount of food ingested, daily available protein intake averaged $65.58 \mathrm{~kJ} / \mathrm{mbm}(\mathrm{SD}=30.78)$ and daily intake of non-protein energy averaged $295.88 \mathrm{~kJ} /$ $\mathrm{mbm}(\mathrm{SD}=163.65)$. According to the time spent feeding, available protein intake averaged 68.3 $\mathrm{kJ} / \mathrm{mbm}(\mathrm{SD}=54.15)$ and daily intake of non-protein energy averaged $186.76 \mathrm{~kJ} / \mathrm{mbm}(\mathrm{SD}=$ 112.53). Linear mixed models showed significant differences in the method used to estimate nonprotein energy and total energy intake, but not protein intake. Thus, feeding time could be considered a good predictor of protein intake. In general, however, our results suggest that feeding time may be a good indicator of foraging effort, but it does not reflect the amount of food or plant preferences. While feeding time captures broad aspects of primate diets, it ignores factors such as processing and handling-time that influence food ingestion and nutrient intake.

\section{A Historical Perspective on Fission-Fusion Dynamics \\ Filippo Aurelia, $b$, Colleen M. Schaffner ${ }^{a}$ \\ aUniversidad Veracruzana, Xalapa, Veracruz, Mexico; 'biverpool John Moores University, Liverpool, UK \\ E-Mail: f.aureli@ljmu.ac.uk; faureli@uv.mx}

Kummer (1971) captured the flexibility in the social systems of hamadryas baboons, geladas and chimpanzees with the term 'fission-fusion societies'. For the first 30 years after its introduction, 'fission-fusion society' was rarely used in publications. In 2004, we organized the symposium 'Fission-Fusion Societies' at the 20th Congress of the International Primatological Society and a post-congress workshop to emphasize the renewed interest in the topic. During the postcongress workshop a critical development occurred when participants agreed that subgrouping through fissions and fusions is a more widespread phenomenon than Kummer's initial proposal. 
In a 2008 Current Anthropology article, workshop participants proposed that any social system can be characterized by its degree of 'fission-fusion dynamics'. This article boosted research on the topic with yearly publications during 2008-2014 tripling compared to the 2004-2007 period. Post-2008 papers using 'fission-fusion societies' were published in numbers similar to those in 2004-2007, whereas publications using 'fission-fusion dynamics' increased substantially in the last three years. The change in citation numbers from before to after 2008 is even a more dramatic. Some of these publications referred to fission-fusion patterns only in passing, whereas others contributed considerably to the expansion of research on the topic. Recent research expands on the frameworks proposed in the 2008 Current Anthropology article to explore the challenges for social interactions and the enhancement of certain communicative and cognitive abilities depending on the degrees of fission-fusion dynamics. Thus, the current situation of research on fission-fusion patterns is quite different from the pre-2008 situation. Based on the continued expansion of this research area, we expect an increasing number of exciting empirical studies as well as phylogenetically controlled comparative studies in the future.

Conservation of Mexican Primates Based on Community Participation Lorena Monserrat Ayala-Camacho ${ }^{a}$, Francisca Vidal-García ${ }^{a}$, Celina Oliva-Uribe ${ }^{b}$, Juan Carlos Serio-Silva ${ }^{a}$

aTecnológico de Estudios Superiores de Huixquilucan, La Magdalena Chichicaspa, bed Biología y Conservación de Vertebrados, Instituto de Ecología A.C., Xalapa, Veracruz, México

E-Mail: biol.monserrat.ayala@gmail.com

The three species of Mexican primates (Alouatta pigra, A. palliata and Ateles geoffroyi) face threats related to the presence of humans because in many regions they are sharing the geographic space with humans. Deforestation, habitat decrease and hunting are the main threats affecting these primate populations. Our objective was to evaluate the perception of local people about primates and about different strategies for primate conservation. We designed a questionnaire and an informative workshop. The questionnaire was applied after and before the informative workshop. Questions were based on knowledge about biology, behaviour and local perceptions, as well as benefits and interest in conservation of primates. The workshop was about two hours long; we discussed with local people the importance of primates and the benefits of different conservation strategies, such as live fences, reforestation and other activities used to promote conservation. We interviewed 499 people in 34 communities in protected and unprotected areas in Tabasco, Chiapas and Veracruz states. We developed a workshop in each community. We identified changes in perceptions and the main interest of people participating in conservation activities. We compared answers after and before the workshop. After the workshop, $99 \%$ of interviewed people changed their opinion and believed it is important to conserve primates and their habitat; $29 \%$ understood that they are dispersal agents; $47 \%$ accepted using live fences, and 53\% accepted doing other activities to protect them; $82 \%$ of people believed that the best place to seed native trees as live fences are the crop fields, because this will let monkeys move between fragments. By knowing the opinion and interests of people sharing the space with non-human primates, as well as their interest in participating in conservation action, we can plan successful actions for primate conservation by involving local people. The ethical issues for carrying out this study were approved and supervised for Comisión Nacional de Áreas Naturales Protegidas (CONANP), and the Instituto de Ecología A.C., Xalapa, Veracruz, México. 


\title{
Evaluating Risk Preferences in Captive Ring-Tailed Lemurs (Lemur catta): A Research Study
}

\author{
Francesca Bandolia , Elisabetta Palagib, c, Paolo Cavicchio ${ }^{a}$, Elsa Addessic ${ }^{c}$ \\ ${ }^{\mathrm{a} G i a r d i n o ~ Z o o l o g i c o ~ d i ~ P i s t o i a, ~ P i s t o i a, ~}{ }^{\mathrm{b}} \mathrm{Centro}$ Interdipartimentale, Museo di Storia \\ Naturale, Università di Pisa, Calci, Pisa, and 'Istituto di Scienze e Tecnologie della \\ Cognizione, Unità di Primatologia Cognitiva e Centro Primati, Rome, Italy \\ E-Mail: francesca.bandoli@zoodipistoia.it
}

The risk-sensitive foraging theory hypothesizes that animals on a positive energy budget should be risk-averse, whereas animals on a negative energy budget should be risk-prone. Species living in a stable environment with consistent food sources are thus predicted to be risk-averse, whereas when resources are not sufficient to satisfy energetic requirements risk-aversion should decrease to enhance chance of survival. Numerous studies have evaluated risk tolerance in monkeys and apes, but only a few have been conducted on prosimians. The present project investigates risk preferences in ring-tailed lemurs (Lemur catta), a prosimian species adapted to stressful and energetically scarce habitats. Our project aims to analyse lemurs' risk preferences in different conditions and to evaluate if they are able to flexibly modify their attitudes toward risk as the probability to obtain the larger reward changes. Preliminary trials were carried out to determine lemurs' quantity discrimination skills, before evaluating their risk preferences in experimental trials. The study is performed in accordance with the European Directive 2010/63/EU.

\section{The Role of Positive Emotions in Cognitive and Communicative Development in Chimpanzees}

\section{Kim A. Bard}

Department of Psychology, University of Portsmouth, Portsmouth, UK

E-Mail: kim.bard@port.ac.uk

Current evolutionary theories of primate social cognition tend to highlight cognition, and mostly ignore emotion. Important social cognitive skills, like joint attention, intentional communication, and social referencing, have developmental and, especially, emotional histories that strongly influence the level of skills acquired. For humans and great apes, histories begin with dyadic engagements in the first 3 months of life. We find significant variation among chimpanzee groups in the number of smiles and amount of mutual gaze with a social partner. Both of these dyadic social outcomes are linked with positive emotional engagements. From 3-8 months, chimpanzees and humans develop emotional reactions to objects and to being tested. Those chimpanzees given more positive engagements respond with higher levels of object manipulation, greater happiness, and less fearfulness during testing, compared to those chimpanzees given more institutional interactional experiences. Chimpanzees show extreme fear of objects when isolated from emotional engagements (Menzel et al., 1970). From 5 to 12 months of life, chimpanzees exhibit joint attention and cooperation, demonstrating triadic coordination between social partners and objects/events. Although these have been thought to be cognitively-based, we found significant and high contributions of positive emotion to both joint attention and cooperation. In the 9-12 month period, for instance, positive emotion accounted for $24 \%$ of the variation in joint attention success, and $33 \%$ of the variation in cooperation. By 1 year of age, chimpanzees given more positive emotional experiences develop a species-typical gestural repertoire, and fewer individuals develop disorganized attachment. Experiencing and expressing positive emotion during development are important proximal factors in social cognition, highlighted in the Lived Experiences Model of Primate Social Cognition. Research was approved by IACUC, Emory University. 


\section{Manipulative Abilities for the Same Tool Use Task in Different Species of Primates}

Ameline Bardo ${ }^{a}$, Antony Borel ${ }^{b}$, Jean Pascal Guéryc, Mathilde Lemaire ${ }^{a}$,Emeline Lempereur ${ }^{a}$, Emmanuelle Pouydebat ${ }^{a}$

aUMR 7179 CNRS-MNHN Paris, and bUMR 7194 CNRS-MNHN Paris, Paris,

'La Vallée des Singes, Romagne, France.

E-Mail: abardo@mnhn.fr

Primates have highly developed abilities for grasping and manipulation that differ between species. In this context, the human hand is considered unique, based on its functional characteristics. However, the real dynamic manual abilities of primates remain poorly known. The purpose of this study is to compare the manipulative strategies in different species of primates (humans, chimpanzees, gorillas, orangutans, capuchins), during the same tool use task. This task requires the use of a tool to recover a static food item in a wooden maze while facing many obstacles; the wire netting between the subject and the maze, and the obstacles inside the maze. All species were in the same experimental conditions allowing us to compare across species. We here focus on the functional strategies used during this task by the different species and quantify the grip types and the in-hand movements involved to reposition the tool in the hand. We found common strategies despite the differences in hand morphology but also strategies specific to some species. We discuss the results in the context of the evolution of manipulative behaviours and highlight the importance of novel methods to understand better the manual specificities of each species. This study complies with the ethical guidelines by the CNRS and French governmental animal care committees and with the IPS Guidelines for the Use of Nonhuman Primates in Research.

\section{Habitat Degradation Affects Gut Microbiota in the Endangered Udzungwa Red Colobus Monkey (Procolobus gordonorum)}

Claudia Barelli ${ }^{a-c}$, Heidi C. Hauffe ${ }^{b}$, Davide Albanese $^{d}$, Massimo Pindo $^{e}$, Claudio Donatid $^{\text {, }}$ Duccio Cavalieri ${ }^{d}$, Francesco Rovero ${ }^{a}$, Kieran Tuohy ${ }^{f}$, Carlotta de Filippo ${ }^{f, g}$

${ }^{a}$ Tropical Biodiversity Section, MUSE - Museo delle Scienze, Trento, and ${ }^{b}$ Department of Biodiversity and Molecular Ecology, Research and Innovation Centre, Fondazione Edmund Mach, San Michele all'Adige, Trento, Italy; 'Reproductive Biology Unit, German Primate Centre, Leibniz Institute for Primate Research, Göttingen, Germany; Departments of ${ }^{\mathrm{d} C o m p u t a t i o n a l ~ B i o l o g y, ~}{ }^{\mathrm{e} G e n o m i c s}$ and Biology of Fruit Crops and ${ }^{\mathrm{f} F o o d}$ Quality and Nutrition, Research and Innovation Centre, Fondazione Edmund Mach, San Michele all'Adige, Trento, and 'Department of Neurosciences, Psychology, Drug Research and Child Health, University of Florence, Florence, Italy

E-Mail: claudia.barelli@muse.it

Gut microbiota are crucial in animal evolution as they affect a number of processes of the host, from digestive efficiency, metabolism, pathogen resistance and immune function. Diversification of gut microbiota composition may derive from either genetic, phylogenetic or from environmental factors. Diets and patterns of nutrient intake can thus change considerably across natural versus human-modified areas. The Udzungwa red colobus monkey (Procolobus gordonorum) is an endangered and endemic primate species living in the Udzungwa Mountains, in the Eastern Arc Mountains in Tanzania, a global biodiversity hotspot. The colobus are primarily arboreal and highly sensitive to hunting and habitat destruction; thus, they provide a useful model for investigating the effect of habitat degradation on gut microbiota diversity. Using barcoded 454 amplicon pyrosequencing 
of the $16 \mathrm{~S}$ bacterial ribosomal RNA gene of 31 faecal samples, we investigated the phylogenetic bacterial diversity of the gut microbiota in seven social groups of free ranging red colobus inhabiting two geographically distinct forests located in the Udzungwas. To understand its variability across habitats with different levels of human disturbances, we analysed 31 faecal samples from either fragmented $(n=16)$ or unfragmented $(n=15)$ forests. Analysis of taxonomy of geographically closely related yet distinct populations of red colobus shows a greater diversification of gut microbiota between groups than within groups and even higher across habitats, with significant differences between groups living in disturbed versus undisturbed forests. We found an increase in biodiversity in the gut microbiota of individuals living in the largest and more undisturbed forest range, correlating with the higher diversity of available food resources. Our results suggest an effect of human disturbance and degradation on gut microbes indicating how strict environmental protection has to be to preserve the natural diversity on a global scale. This research complied with the International Primatological Society (IPS) Guidelines for the Use of Nonhuman Primates in Research.

\title{
Food, Sex and Violence: A Search for the Drivers of Social Organization in the Genus Cacajao
}

\author{
Adrian Barnett ${ }^{a, b}$, Sarah Boyle $e^{c}$ \\ aCentre for Research in Evolutionary and Environmental Anthropology, Department of Life \\ Sciences, University of Roehampton, London, UK; biodiversity Research Unit, National \\ Institute for Amazonian Research, Manaus, Amazonas, Brazil; 'Department of Biology, \\ Rhodes College, Memphis, Tenn., USA \\ E-Mail: adrian.barnett1.biology@gmail.com
}

Uacaris (genus Cacajao) are Neotropical primates with a diet dominated by unripe fruit and seeds, and a fission-fusion social system. They are restricted to the western Amazon basin, where their range coincides with gradients in soil fertility, duration and extent of rainy season, plant species diversity, fruit production, food tree dispersion and availability of dry-season fall-back foods. All of these link to resource availability. Of the eight recognised taxa in the genus Cacajao, four have been studied intensively and these show notable variation in such social factors as levels of aggression, male-male bonding and proximity when foraging. To test the null hypothesis that these social factors are not associated with environmental (resource availability) factors, nor linked to other aspects of social organisation, we conducted a principle component analysis and tested for correlation between the independent variables with a Pearson Correlation Matrix. In addition to social and resource-linked factors listed above, we included the following variables: duration of reproductive availability in females, mating system type, grooming frequency, and use of fall-back foods such as insects and leaves. We found correlations between such environmental factors as phenological patterns, reproductive periodicity and aggression frequency, and between male-male sociology, foraging proximity and regional productivity. However, the exact composition and directionality of the cause-and-effect links is not yet clear, partially due to small sample size and samples being taken from different years. Long-term, coordinated data collection is clearly required. 


\section{Concealed Insectivory: Seed-Dwellers and Stem-Borers in the Diet of a Specialist Seed Predator, the Golden-Backed Uacari, Cacajao ouakary}

Adrian Barnett ${ }^{a, b}$, Peter Shaw ${ }^{c}$, Ann MacLarnon $^{a}$, Wilson Spironello ${ }^{b}$, Caroline Ross ${ }^{a}$

${ }^{a}$ Centre for Research in Evolutionary and Environmental Anthropology, Department of Life Sciences, University of Roehampton, London, UK; ${ }^{b}$ Biodiversity Research Unit, National Institute for Amazonian Research, Manaus, Amazonas, Brazil; 'Centre for Research in Ecology, Department of Life Sciences, University of Roehampton, London, UK E-Mail: adrian.barnett1.biology@gmail.com

Fruits and seeds are often infested with insects and nematodes. Potentially, these offer a substantial source of animal protein, as well as vitamins and micronutrients, for frugivorous vertebrates. But their importance is little-studied. We report on the foraging behaviour of the specialist unripe fruit and seed predator, the golden-backed uacari, Cacajao ouakary, in relation to the invertebrate content of the fruits it ate. The uacari ate fruits of 101 species of plant, of which 26 showed invertebrate infestation. All infesting invertebrates were insects. Comparison of the frequency of infested fruits in diet remnants and on the trees showed that uacaris were selecting infested fruits in 16 species and avoiding them in three species. The selection of infested fruits was not due to uacaris selecting for mechanical advantage offered by a pericarp weakened by insect burrows. As larvae are commonly $60-80 \%$ protein, and up to $5.5 \mathrm{~g}$ of larvae were retrieved per fruit, selection is attributed to protein gain. This effect may be important for understanding primate foraging decisions, and we suggest that diet sampling methodologies should be adjusted to include this foraging mode.

\section{From Ear to Lifestyle: Correlation Between Ear Morphology and Primate Ecology}

Margot Bernardi, Sébastien Couette

UMR uB/CNRS Biogéosciences, University of Burgundy, Dijon, France

E-Mail: margot_bernardi@etu.u-bourgogne.fr

Primate morphological variation has been studied for many centuries. Morphology is largely used to understand differences between species and to identify adaptations to specific environments. Well-preserved parts, such as teeth or cranial and post-cranial elements, may provide significant functional and phylogenetic information. Basicranial parts, especially the ear, are often used to propose phylogenetic hypotheses. Nowadays, developments in high-resolution imaging allow the internal structure of the ear to be explored. Since the ear is the centre of auditory capacity and balance, ear studies provide new insights into hearing and locomotion. Previous works have already used $\mu \mathrm{CT}$ data to study the relationships between ear morphology and function. However, intraspecific variation is almost never quantified. In this study, we measured 13 morphological variables of the middle and inner ear. These parameters were quantitatively analysed to compare intra- and inter-specific variation in extant strepsirrhines. Our morpho-functional results were then compared to ecological and behavioural data from the literature. Some significant correlations can be found between ear morphology and life history traits. Thus, ear morphology is a precious tool to assess ecological and behavioural characteristics. Based on our results, we propose a reconstruction of the palaeo-environment and palaeo-ecology of a fossil species from the Eocene. 


\title{
Habitat Use and Spatial Niche Partitioning of Five Nocturnal Primates in Angola
}

\author{
Elena Bersacola, Magdalena S. Svensson, Simon K. Bearder
}

Nocturnal Primate Research Group, Oxford Brookes University, Oxford, UK

E-Mail: hellenbers@gmail.com

Eighteen species of galagos and three species of pottos are currently recognised and in several regions the distributions of these strepsirrhines overlap. Niche partitioning is a key factor in determining community structure as it allows the coexistence of two or more species that rely on limited trophic resources. For this study, we aimed to investigate patterns of coexistence and habitat use of nocturnal primates in Angola. We conducted surveys at four study sites, representing main habitat types in Angola: Kumbira Forest Reserve (moist, secondary forest), Bimbe (semi-arid savannah woodland), Northern Scarp (moist, primary and secondary forest) and Calandula (miombo woodland/gallery forest mosaic). At each animal encounter, we recorded species, number of individuals, height of animals above ground and GPS location. We assessed vegetation characteristics using the point-centered quarter method. At each point we also collected data on habitat disturbance level, undergrowth density and canopy cover. We observed four galago species and one potto, with community structure differing across sites. Dwarf galagos (Galagoides spp.) were significantly more abundant in moist forests and, in Calandula, Demidoff s galago (G. demidovii) appeared to be restricted to gallery forest. Mohol galago ( $\mathrm{Ga}$ lago moholi) occurred only in miombo woodland/gallery forest mosaic, and the potto (Perodicticus potto) was only present in moist forests. Abundance of Demidoff's galago correlated with density of undergrowth vegetation. In sites where abundance of species was high all the strepsirrhines had a strong vertical separation in the use of vegetation, and in the other sites the niche partitioning was more related to habitat type. We hope this study will contribute as a baseline for future conservation work and research on these cryptic primates. Our research complied with the International Primatological Society (IPS) Guidelines for the Use of Nonhuman Primates in Research.

\section{Feeding Behaviour in Chimpanzees at Caiquene-Cadique, Guinea-Bissau: From Crops to Snails}

\author{
Joana Bessa ${ }^{a}$, Cláudia Sousa ${ }^{t, a}, b$, Kimberley J. Hockings ${ }^{a, c}$

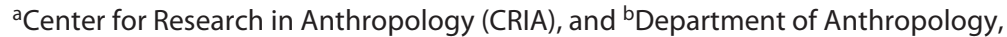 \\ Faculdade de Ciências Sociais e Humanas, Universidade Nova de Lisboa, Lisbon, Portugal; \\ 'Anthropology Centre for Conservation, Environment and Development, Oxford Brookes \\ University, Oxford, UK \\ ${ }^{\dagger}$ Passed away September 29, 2014 \\ E-Mail: joana.h.bessa@gmail.com
}

With rising conversion of 'natural' habitat to other land use, such as agriculture, nonhuman primates are increasingly exploiting areas influenced by humans. Despite the conservation importance of understanding how primates modify their behaviour in response to human pressures, data are lacking, even for well-studied species. In human-influenced environments, understanding a species' diet is one of the best ways to assess their ability to cope with changing conditions, e.g. exposure to novel food sources. Using 9 months of systematically collected data (faecal samples, feeding traces and direct observations), we examined the feeding ecology of an un-habituated community of western chimpanzees (Pan troglodytes verus) inhabiting a forest-savannahmangrove-agricultural mosaic at Caiquene-Cadique, Cantanhez National Park, Guinea-Bissau. 
There was seasonal variation in the availability of chimpanzee food species in the apes' home range, but a constant high proportion of ripe fruit in their diet. Ten important food species were identified, including Elaeis guineensis fruit and flower. In addition to wild plant foods, agricultural crops were regularly consumed, representing $13.6 \%$ of all species eaten. Honey was also frequently consumed, but there was no confirmation of insects or vertebrates being taken. We found indirect evidence of possible smashing and consumption of giant African snails (Achatina sp.), but the importance of this behaviour could not be determined. Caiquene-Cadique represents one of three sites (including Bili in DRC and Nimba in Guinea) where this behaviour might occur. Human-chimpanzee 'conflicts' over access to space and resources are rare in CaiqueneCadique, but likely to become more commonplace with growing human populations. For biodiversity conservation to work in areas where humans and wildlife co-exist, the needs of both have to be incorporated into locally-appropriate flexible strategies. Research complied with the European Directive 2010/63/EU.

\section{Quantitative Approaches to the Study of Emotions in Non-Human Primates: Lessons from Human Cognitive Neuroscience \\ Emily Bethell ${ }^{a}$, Caralyn Kemp ${ }^{a}$, Amanda Holmes ${ }^{b}$, Ann MacLarnon ${ }^{b}$, Stuart Semple \\ aLiverpool John Moores University, Liverpool, and ${ }^{b}$ University of Roehampton, London, UK \\ E-Mail: E.J.Bethell@ljmu.ac.uk}

In humans it is well documented that differences in trait emotional predisposition and changes in emotion state are characterised by respective differences and changes in cognitive processes. These emotionally mediated shifts in cognition are known as cognitive biases. Cognitive biases are widely associated with psychological wellbeing in humans, and are implicated in the onset and maintenance of human psychological disorders. For example, socially anxious people avoid looking at social stimuli such as emotional faces and interpret ambiguous cues more negatively than do non-anxious people. In this talk I will present data from the first studies to adapt human cognitive bias measures for use with non-human primates. I will discuss the role of affect in mediating attention to social stimuli and in influencing interpretation of ambiguous stimuli. I will then discuss challenges and future directions for this new approach to the study of non-human primate emotions. This research complied with the European Directive 2010/63/EU and the International Primatological Society (IPS) Guidelines for the Use of Nonhuman Primates in Research.

\section{Whose Honk? Using Acoustic Playbacks to Test Species Recognition in Galagos}

Caroline M. Bettridge, Selvino R. de Kort

Conservation, Ecology and Behaviour Research Group, Manchester Metropolitan

University, Manchester, UK

E-Mail: c.bettridge@mmu.ac.uk

Galago species have distinctive, varied vocal repertoires that have been used to establish species boundaries and describe new species, yet there are no studies on how galagos themselves respond to, or discriminate among, these different calls. For example, one species reclassification based partly on vocalisation characteristics was the separation of the southern Galago moholi from the more northerly and widely distributed G. senegalensis. We use acoustic playback meth- 
ods to investigate differences between behavioural responses of $G$. senegalensis sotikae in Tanzania to calls of their own and other G. senegalensis subspecies and of the distinct species, Galago moholi. Social contact calls were played in a controlled, paired experimental design to a population at Kwakuchinja-Mbugwe in northern Tanzania. Galagos responded to playbacks of contact calls by approaching the speaker, vocalizing and scent marking. Individuals responded more strongly and more rapidly to calls of their own versus a different subspecies. Contact calls were given only in response to calls of their own subspecies, whereas scent marking was recorded only in response to playbacks of a different subspecies. There was little to no obvious behavioural response to the calls of G. moholi. There may be a seasonal effect on calling behaviour with observed differences in responses between the two field seasons. These studies are the first evidence of discrimination between con- and heterospecific calls by galagos and may provide a new technique for identifying or classifying species in the future. Research was conducted in collaboration with CAWM, Tanzania and with the kind permission of TAWIRI. The study complied with the International Primatological Society (IPS) Guidelines for the Use of Nonhuman Primates in Research.

\title{
Home Sweet Home (Range): A Multi-Annual Study on Indri indri Spatial Behaviour
}

\author{
G. Bonadonna ${ }^{a}$, V. Torti ${ }^{a}$, M. Gamba ${ }^{a}$, R.M. Randrianarison ${ }^{b}$, C. Giacoma ${ }^{a}$ \\ aDepartment of Life Science and Systems Biology, University of Turin, Torino, Italy; ${ }^{b}$ GERP, \\ Group d'étude et de recherche sur les primates de Madagascar, Antananarivo, Madagascar \\ E-Mail: giovanna.bonadonna@unito.it
}

One of the basic requirements in the study of a species is an understanding of its relationship with the environment, determined by taxon-specific ecological, physiological and social factors. The spatial context of a species is well defined by the quantitative description of its home range (HR), defined as the spatial context in which a group or an animal habitually feeds, moves and rests. Furthermore, knowledge of an animal's home range is important in planning conservation strategies. When a HR is actively defended we can refer to it as a territory. This is a multiannual study of spacing behaviour of Indri indri, a threatened, social monogamous lemur that lives in groups of 2-6 individuals occupying exclusive territories defended from conspecific intruders. We tested whether: a) the groups maintain territorial stability over time, b) they have exclusive HRs, and c) songs have inter-group spacing function. To do that, we estimated the HR size and stability, and the ranging behaviour of 8 groups of Indri indri over a minimum of 3 consecutive years at 3 different sites in Madagascar. We also compared the HR size estimated with different methods and the sampling efforts needed to obtain reliable results. Indris occupy relatively small territories $(11.60 \pm 3.10$ ha MPC $-15.57 \pm 2.84$ ha Kernel). Inter-group overlaps at the territorial borders varied from $1.74 \%$ to $5.33 \%$ of their HRs, and were stable over time. Intergroup encounters were rare and occurred at territory boundaries. Disputes lasted from a few minutes to hours and were always accompanied by vocal exchanges characterised by alternating songs from the two groups, emission of aggressive vocalizations, marking and, sometimes, by physical fights. The use of loud acoustic signals diminished the risk of direct physical encounters and allowed effective mate guarding, as is the case in other monogamous mating systems. This study complied with the European Directive 2010/63/EU. Research permit No. 066/14/MEF/SG/ DGF/DCB.SAP/SCB issued by Madagascar MEF. 


\title{
Gene Flow and Genetic Diversity of Tanzania's Greater Mahale Ecosystem Chimpanzees
}

\author{
Noémie Bonnin ${ }^{a}, b$, Alex K. Piel ${ }^{b}$, Miguel. A. Ramirez', Yingying Lic, D. Elizabeth Loy', \\ Patricia Crystal', Beatrice Hahn', Leslie A. Knapp ${ }^{d}$, Fiona A. Stewart ${ }^{b}$

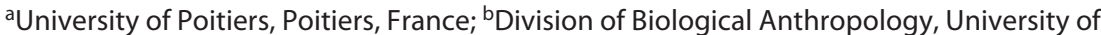 \\ Cambridge, Cambridge, UK; ${ }^{\mathrm{C}}$ Departments of Medicine and Microbiology, University of \\ Pennsylvania, Philadelphia, Pa., and dDepartment of Anthropology, University of Utah, \\ Salt Lake City, Utah, USA \\ E-Mail: noemiie.bonnin@gmail.com
}

Over $90 \%$ of Tanzania's chimpanzees are estimated to live within the $19,400 \mathrm{~km}^{2}$ Greater Mahale Ecosystem (GME) and most ( 75\%) are on unprotected land. Human population expansion and concurrent regional deforestation threaten these chimpanzees and the habitat in which they live. The loss, degradation and fragmentation of suitable habitat can impede animal movements, reducing potential for dispersal and influencing genetic population structure and viability. We therefore investigated chimpanzee population structure and gene flow within the GME. Between July 2011 and September 2012 we collected 756 chimpanzee faecal samples from 11 target areas and one long-term field site (Issa, Ugalla). Following successful extraction of DNA from faeces, we determined individual genotypes by multiplex polymerase chain reactions (PCR) to amplify eight to twelve highly polymorphic autosomal microsatellite loci. We amplified and sequenced a $498 \mathrm{bp}$ fragment of the mitochondrial D-loop (hypervariable region one). We grouped sequences into haplotypes and subjected them to phylogenetic analysis in comparison to available published Tanzanian chimpanzee D-loop sequences. We identified 320 individuals and 13 haplotypes. We used Arlequin 3.1 to calculate nucleotide diversities, pairwise FST values, and to investigate population differentiation in relation to geographic distance within the GME and Tanzania. We discuss population structure and gene flow within the GME in reference to natural barriers, such as rivers, and to human disturbance, such as human settlements. Understanding current, as well as historical, patterns of gene flow within and between populations has important implications for chimpanzee conservation and for prioritising areas to preserve population connectivity in an increasingly anthropogenically disturbed landscape.

\section{Human Influence on the Activity Pattern of the Common Marmoset (Callithrix jacchus) in the Botanic Garden, João Pessoa, Paraíba, Brazil}

Noémie Bonnin ${ }^{a}$, Jean-Baptiste Decotte ${ }^{b}$, Carla Soraia Soares de Castro ${ }^{c}$

anniveristy of Poitiers, Poitiers, and bPractical School of High Study, Paris, France;

'Federal University of Paraíba, João Pessoa, Brazil

E-Mail: noemiie.bonnin@gmail.com

The expansion of humans is threatening biodiversity. Aspects like habitat loss and fragmentation are major threats to terrestrial biodiversity. Although fragmentation is a world-wide phenomenon, tropical rain forests, in particular, have suffered from it. In Brazil, the Atlantic forest in the northeast is vanishing and the fauna living there has to adapt. Social animals such as primates are good indicators of the disturbance caused by humans. This paper describes the impact of humans on the activities of common marmosets (Callithrix jacchus) living close to human pressure in the Botanic Garden in João Pessoa city, Paraíba, Brazil. One marmoset group was observed from April to July 2013 (three days a week). We tested the hypothesis that being offered food by humans impacts the aggressiveness of the group's members as well as their spatial distribution. The Instantaneous Scan Sampling Method was used to record the marmosets' behaviours, and their spatial distribution was recorded using a GPS. During the observation time, the mar-

6th European Federation for Primatology Meeting
Folia Primatol 2015;86:235-386 DOI: $10.1159 / 000435825$ 
mosets spent the greatest proportion of their time eating (48\%), while the rest of the time was divided into travelling (22\%) and social behaviour (21\%). Their diet was composed mostly of gum (50\%), fruits (30\%) and animal prey (18\%), but they were also recorded eating human foods (2\%), such as biscuits, crisps and bread. We discuss the results in comparison with available published literature on the activity budgets of wild groups of $C$. jacchus. In addition to the destruction of the habitat, human activities may be responsible for a true change in the behaviour and life of wildlife.

\author{
What Characterizes a Successful Initiator? A Case Study in Two \\ Macaque Species \\ Céline Bret ${ }^{a-d}$, Jean-Louis Deneubourg ${ }^{c}$, Odile Petit ${ }^{a-c}$ \\ aDépartement Ecologie, Physiologie et Ethologie, Centre National de la Recherche \\ Scientifique, and ' Institut Pluridisciplinaire Hubert Curien, Université de Strasbourg, \\ Strasbourg, France; 'Unit of Social Ecology, Free University of Brussels, Brussels, Belgium; \\ ${ }^{\mathrm{d}}$ German Primate Centre, Junior Research Group Primate Sexual Selection, Göttingen, \\ Germany \\ E-Mail: CBret@dpz.eu
}

In many species, the mechanism underlying decision-making during collective movements is based on some specific group members that are more successful in leading their group through the environment. The characterization of the success of an individual in being followed by other group members is crucial to understanding why these individuals are so important for group decisions. Several variables have been used in the literature; however, they discriminate an initiator's success differently depending on the species under consideration. In this study, working on semi-free ranging groups of two closely related primate species, Macaca tonkeana and M. mulatta, we compared the two most used variables, the number of followed initiations per individual and the ratio per individual between the number of followers and the number of potential followers at the time of initiation. We found that in Tonkean macaques, some individuals performed more followed initiations than others, but when initiating, leaders were frequently followed by the entire group. In contrast, rhesus macaques exhibited less-marked differences in the propensity to initiate followed movements, but some individuals were more successful in term of number of followers than others. In order to preserve these differences in leaders' success, we propose a success index combining the two variables, which could be used in both species and thereby allow inter-specific comparison. Our research was non-invasive and complied with the European Directive 2010/63/EU and the International Primatological Society Guidelines for the Use of Nonhuman Primates in Research.

\title{
The Movement Is Played by the Quatuor: Keystone Individuals Lead Group Movements in White-Faced Capuchins (Cebus capucinus)
}

Léa Briard ${ }^{a-c}$, Odile Petit ${ }^{a-c}$, Jean-Louis Deneubourg ${ }^{b, c}$

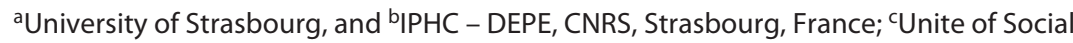

Ecology, Free University of Brussels, Brussels, Belgium

E-Mail: lea.briard@iphc.cnrs.fr

A keystone individual is defined as an individual that has a disproportionally large effect on the other group members and/or on the overall group dynamics (Sih \& Watters 2005). What characterizes a keystone individual depends on the study species and context. In the context of 
collective decision-making, these keystone individuals are sometimes referred as 'leaders'. Leadership in white-faced capuchins has been studied for several years and has been classified as distributed. However, although all group members can initiate collective movements, success may not be equally shared. In this context, some individuals may be more successful than others, which may suggest the existence of keystone individuals able to drive group movement. We studied a semi-free ranging group of 10 white-faced capuchins over 2 years (1999-2000) and recorded more than 300 events of collective movements. Social status (e.g. centrality, hierarchical rank), age and kinship were used as predictors of individual success. By studying the individual profiles of success when initiating, we found that 4 individuals were responsible for most of the collective movements involving the entire group and could therefore be deemed as super-initiators, i.e. keystone individuals. However, none of them seemed to share a common characteristic suggesting that keystone individuals could possess different characteristics or that we need to look for other common parameters such as personality. Our research complied with the International Primatological Society Guidelines for the Use of Nonhuman Primates in Research.

\title{
Monkey Business: Inter-Group Differences in the Object/Food Bartering Practice in Balinese Macaques (Macaca fascicularis) at the Uluwatu Temple, Indonesia
}

\author{
Fany Brotcorne ${ }^{a, b}$, Jean-Baptiste Leca ${ }^{b}$, Noëlle Gunst ${ }^{b}$, I. Nengah Wandia ${ }^{c}$, Agustin Fuentes ${ }^{d}$, \\ Marie-Claude Huynen ${ }^{a}$
}

aBehavioural Biology Unit, University of Liège, Liège, Belgium; 'bepartment of Psychology, University of Lethbridge, Lethbridge, Alta., Canada; 'Primate Research Centre, Universitas Udayana, Bali, Indonesia; ${ }^{\mathrm{d} D e p a r t m e n t}$ of Anthropology, University of Notre Dame, Notre Dame, Ind., USA

E-Mail: fbrotcorne@gmail.com

While there is increasing evidence for the social transmission of behavioural innovations and intergroup behavioural variation in a wide range of nonhuman primate taxa, some behavioural domains (e.g., tool use) are far more represented than others (e.g., arbitrary social conventions) in the literature. Our study explores the 'object/food bartering' activity in the free-ranging long-tailed macaques, Macaca fascicularis, living around the Uluwatu temple, southern Bali (Indonesia). This practice occurs in two steps: after robbing temple visitors of non-edible objects, the monkeys use these objects as tokens, by returning them in exchange for specific food rewards. This spontaneous population-level activity is customary and enduring at Uluwatu, whereas it is very rare or absent at other macaque-tourism sites across the island. During a fourmonth study in 2010 at Uluwatu, we used the all-occurrence sampling technique to record 186 successful events of object-robbing, where 95 (51\%) were followed by object/food bartering attempts. In line with the 'needing-to-learn' hypothesis, we found that older individuals were significantly more efficient at robbing valuable objects and more successful at exchanging them for food than younger individuals. We also found substantial differences in the frequency and form of the bartering practice ( $\mathrm{n}=95$ events) among the four social groups constituting the Uluwatu population, with two groups ('Temple': $60 \%$ of the bartering events, 'Tear': $34 \%$ ) being responsible for more frequent bartering events than the two other groups ('Scarface': 4\%, 'Nez': 2\%). We investigated the role of group-specific environmental and anthropic influences (food provisioning and degree of human presence) in such intergroup differences. Taken together, these preliminary results suggest that the bartering practice could be a local behavioural tradition in Balinese macaques. 


\title{
Gastrointestinal Parasite Communities in Mountain Gorillas (Gorilla beringei beringei) as a Conservation Tool for Tourism and Community Development in the Virunga Mountains
}

\author{
J. Brunner ${ }^{a}$, J. Rothman ${ }^{b}$, M. McLennan ${ }^{a}$, M. Cranfield', T. Gillespie ${ }^{d}$

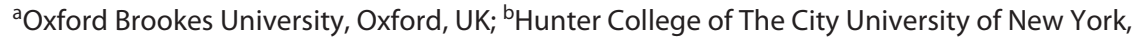 \\ New York, N.Y., USA; 'Gorilla Doctors, Musanze, Rwanda; 'DEmory Global Health Institute, \\ Atlanta, Ga., USA \\ E-Mail: jaysen.brunner-2014@brookes.ac.uk
}

The mountain gorilla, Gorilla beringei beringei, is endemic to just two small populations in Uganda, Rwanda and the Democratic Republic of Congo. Key threats include habitat loss, political instability, poaching and, above all, disease. Mountain gorillas are highly susceptible to anthropozoonotic disease transmission due to their proximity to local communities/tourists and high relatedness to humans. Many parasite studies have been conducted in the Bwindi population, but recent data are lacking for the Virunga population. Faecal samples from wild and captive gorillas in the Virunga Mountains in Uganda, Democratic Republic of Congo and Rwanda were collected over a three-month period and examined for intestinal parasite presence via flotation and sedimentation. We analysed the potential role(s) that habituation, home range and captive management can play in host-parasite ecology. Parasites were identified to the genus/ species level where possible, and parasite community richness was compared among gorilla groups. Results were disseminated to Gorilla Doctors, a group of veterinarians working in mountain gorilla and Grauer's gorilla (Gorilla beringei graueri) habitats. Combined with data on respiratory disease and bacterial infection, these parasitological analyses will effectively inform Gorilla Doctors of the diseases infecting this endangered gorilla population. Ultimately this will aid in treating infected groups, and evaluating current tourist practices. The methods used comply with the International Primatological Society Guidelines for the Use of Nonhuman Primates in Research.

\section{Where Do Bossou Chimpanzees Spend Their Time? Ranging Patterns and Activity Budget Allocation in Relation to Habitat Types, Resource Availability and Anthropogenic Influences}

\author{
Nicola Bryson-Morrison ${ }^{a}$, Tatyana Humle ${ }^{a}$, Tetsuro Matsuzawa ${ }^{b}$ \\ aUniversity of Kent, Canterbury, UK; ' bPrimate Research Institute, Kyoto University, Inuyama, \\ Japan \\ E-Mail:nb258@kent.ac.uk
}

Studies that examine movement patterns and use of heterogeneous environments are vital for understanding the resource and habitat requirements of primate populations. Determining the use of different habitat types at fine spatial scales, along with the ecological, anthropogenic and behavioural factors that drive this use, can help identify habitat preferences for prioritisation in conservation management decisions. The aim of our study was to quantify resource and habitat selection and ranging patterns of a chimpanzee community in the heterogeneous anthropogenic landscape of Bossou, Guinea, West Africa. Specifically, we examined activity budget allocation in all habitat types and the influence of resource availability and anthropogenic factors on habitat use. Our study was conducted from April 2012 - March 2013. We collected 15 min behavioural scan data as well as GPS ranging locations every $10 \mathrm{~m}$. We conducted phenology and 
food species availability surveys for all habitat types and mapped all anthropogenic features. We found that the chimpanzees used habitat types within their home range differently, spending more or less time engaged in specific behaviours than expected for particular habitats. Resource availability and anthropogenic factors, such as nearest distance to cultivated fields, influenced chimpanzee ranging and habitat use. Chimpanzees did not use each available habitat type equally and each differed in terms of resource availability and anthropogenic influences. Our results highlight the importance of defining fine scale ranging patterns and home range use of a heterogeneous environment to promote our understanding of the ecological and anthropogenic factors that influence the suitability of anthropogenic landscapes for the long term survival of chimpanzees. Our research complied with the European Directive 2010/63/EU and with protocols approved by the Guinean Ministry of Education and Scientific Research authorities.

\title{
At What Cost Do Capuchin Monkeys Willingly Share Food with a Conspecific?
}

\author{
Benoit Bucher, Hika Kuroshima, Kazuo Fujita
}

Department of Psychology, Graduate School of Letters, Kyoto University, Kyoto, Japan

E-Mail: bucherbenoit@live.fr

Although prosocial behaviours are commonplace in human societies, their cognitive mechanisms and evolutionary roots are yet to be understood. Studies of pro-sociality in great apes, involving food sharing tasks, suggest that altruistic food sharing may be a uniquely human characteristic (Tian et al., 2013; Bullinger et al., 2014). However, tufted capuchin monkeys (Cebus apella), a New World species which diverged from apes about 40 million years ago, have been shown to be sensitive to others' welfare (Takimoto et al., 2010), remain untested on this question of altruism. To investigate capuchins' food sharing capacities, 12 pairs composed of a benefactor and a partner were tested in two adjacent compartments. We observed whether the benefactor monkeys (those in possession of food) would benefit their partner by voluntarily delivering them food. In the first experiment, the cost of sharing was high; benefactors had to allow their partner to eat a portion of their own food (altruistic sharing). In the second experiment, the cost was reduced as the benefactors no longer had to share their own food but an inaccessible one (active giving). The results suggested that capuchins were unwilling to share food altruistically. However, when the cost of sharing decreased, they sometimes chose to act pro-socially toward selected individuals. The results will be discussed in line with previous ape studies reporting that partners' behaviours may also play an important role for pro-sociality to appear, by driving the benefactor's response (Yamamoto et al., 2009). The experimental procedures received approval from the Animal Experimentation Committee of the Graduate School of Letters, Kyoto University.

\section{When Is Behaviour Intentional? The Case of Proactive Prosociality \\ Judith M. Burkart, Conrad Roelli, Ruben Richiger}

Anthropological Institute and Museum, University of Zurich, Zurich, Switzerland

E-Mail: Judith.Burkart@aim.uzh.ch

The question of whether a behaviour represents a goal-directed act or an involuntary reaction has mainly been addressed in the context of communication. However, it is relevant for a much larger range of behaviours, in particular those that may represent evolutionary precursors to presumably derived human traits. In the first part, we will focus on prosocial behaviour in 
common marmosets, and explore to what extent it satisfies criteria of intentionality typically used in communication research. We show that marmoset prosocial behaviour (i) shows some degree of flexibility, with individuals using multiple means to reach their goal, (ii) cannot be explained by alternative mechanisms such as conditioning processes, (iii) continues until the putative goal is reached, and (iv) that prosocial behaviours that don't lead to the expected outcome elicit distinct reactions in the actor. Even though this evidence is consistent with an intentional description, potential killjoy explanations remain possible because this approach can never provide positive evidence for the absence of intentional behaviour. We therefore propose an alternative approach that is able to distinguish whether individuals understand the behaviour of others in terms of goal-directedness or not, and use it to demonstrate that marmosets are indeed able to do so. Such a combined approach may be most reliable in detecting intentional behaviour. The experiments complied with the European Directive 2010/63/EU and were approved by the Kantonales Veterinäramt Zürich, license No. 183/2013 and No. 150/2010.

\title{
Ecological Factors Affecting Fission Decision in Wild Spider Monkeys (Ateles geoffroyi)
}

\author{
Laura Busia ${ }^{a}$, Colleen M. Schaffner ${ }^{a}$, Jessica M. Rothman ${ }^{b}$, Filippo Aurelia \\ anstituto de Neuroetologia, Universidad Veracruzana, Xalapa, Veracruz, Mexico; \\ bepartment of Anthropology, Hunter College of the City University of New York, \\ New York, N.Y., USA \\ E-Mail: laura.busia@gmail.com
}

Two key factors affecting grouping patterns in primates are resource availability and predation risk. For example, individuals can live in larger groups when higher resource availability reduces competition among them. Similarly, group members are less vulnerable to predation in larger groups. In species characterized by a high degree of fission-fusion dynamics, individuals are found in subgroups that change size and composition several times a day through fission and fusion events. The study of a single group of these species allows comparisons between subgroups of different size without the need to study multiple groups. The aim of our study was to examine how resource availability and predation risk affect grouping patterns in spider monkeys (Ateles geoffroyi), a species characterized by a high degree of fission-fusion dynamics. In this species food competition is reduced by adjusting subgroup size to food availability. Although spider monkey predation pressure is relatively low compared with other primate species, the individuals' perception of predation risk may result in them being in larger subgroups. From January 2013 to September 2014 we collected data on 23 adult and subadult individuals of a spider monkey community living in the Natural Protected Area of Otoch Ma'ax Yetel Kooh, Yucatan, Mexico. We recorded data on fission events, food characteristics and individuals' perception of predation risk. A fission event was recorded when an individual moved at least $30 \mathrm{~m}$ away from a subgroup member for more than $30 \mathrm{~min}$. Concerning food characteristics, we estimated the fruit abundance of trees on which subgroups foraged and we determined the food quality through macronutrient analyses of the most consumed fruit species. Alarm calls were used as indicators of individuals' perception of predation risk. Using these measures of food characteristics and perception of predation risk, we tested predictions about individual fission decisions and changes in subgroup size. 


\title{
Gimme More: Exudates Do Not Characterise a Fallback Food in the Diet of the Javan Slow Loris (Nycticebus javanicus)
}

\author{
Francis Cabana, K.A.I. Nekaris
}

Nocturnal Primate Research Group, Oxford Brookes University, Oxford, UK

E-Mail: 13123873@brookes.ac.uk

Gum has been identified as a fallback food for pygmy and greater slow lorises even though their entire physiology and morphology is adapted to harness this food source. In order to test this hypothesis empirically, wild feeding data must be collected not only by instantaneous observations but also by quantitative analysis. We observed wild Javan slow lorises (Nycticebus javanicus) as a model species, at our field site on Mt. Papandayan, West Java, Indonesia, from June 2014 to June 2015. Feeding behaviour was recorded every 15 min to create an activity budget, however, all occurrences of feeding observations and intake amounts were also documented. Gum was recorded in seconds, nectar by number of flowers eaten, fruits by fractions and part of fruit eaten, and insects by frequency. To calculate actual food intake, gum intake trials were later conducted at a rescue centre in West Java, Indonesia and volume and weight of nectar per flower were determined through microcapillary absorption. Phenology of five random plots was monitored monthly to assess food availability and samples of each food item were collected and analysed for nutritional content. Food preference was calculated. Using instantaneous sampling, gum eating was observed $49.62 \%$ of the time, insects $10.55 \%$, nectar $29.34 \%$, fruit $2.37 \%$ and other plant matter $8.12 \%$ (young bamboo leaves, flowers and sap). However, average nightly weighted food intake on a fresh weight basis revealed different figures, with insects making up the highest intake at $33.02 \%$, followed by fruit $30.88 \%$, gum $26.41 \%$, flowers $3.95 \%$, young bamboo leaves $3.17 \%$ and nectar $2.57 \%$. Nutritional geometry was used to determine the importance of gum and bamboo leaves as a source of nutrients within the lorises' diet. There was no significant difference in the ingestion of gum in the dry or wet season even if gum was more available during the wet season. With results thus far, gum does not appear to be a fallback food, but instead a dietary staple which balances the nutrient profile of the wild $N$. javanicus diet. The research complied with the International Primatological Society (IPS) Guidelines for the use of Nonhuman Primates in Research and has been approved by Oxford Brookes University Ethical Committee.

\section{Probability of Behaviours Potentially Indicative of Stress in Behavioural Sequences of Captive Capuchin Monkeys}

Carolina Pereira Cadório, André Campos Pereira, Mariana Mascarenhas Winandy, Vanessa Coelho de Lima, Nicole Galvão-Coelho, Renata Gonçalves Ferreira

Universidade Federal do Rio Grande do Norte, Natal, Rio Grande do Norte, Brazil

E-Mail: carolina_cadorio@yahoo.com.br

Pacing, self-grooming, bouncing, crouching, head twirl, ingestion of faeces and sexual display are behaviours commonly used as potential indicators of stress (BPIS) in captive animals. However, the use of BPIS is equivocal since percentages of BPIS do not always correlate to physiological indicators of health and stress. Alternatively, it has been suggested that analysis of sequences and transition of behaviour offer a more fine grained approach. During $147 \mathrm{~h}$ we studied 25 capuchin monkeys: 6 Sapajus libidinosus maintained in $24 \mathrm{~m}^{3}$ non-enriched enclosures (CETAS, Natal - RN), 6 S. flavius kept in enriched enclosures of $120 \mathrm{~m}^{3}$ (Zoo of Salvador - BA) and 13 S. xanthosternos held in an enriched enclosure of $120 \mathrm{~m}^{3}$ and on an island of $100 \mathrm{~m}^{2}$ (Salvador Zoo). Every $30 \mathrm{sec}$, during $10 \mathrm{~min}$ of focal animal sampling, we recorded the behaviours according to an ethogram of 9 macro-behavioural categories and 7 BPIS. After employing transitions matrix analysis of probability $\left(\mathrm{P}_{(\mathrm{i}, \mathrm{j})}=\mathrm{N}_{(\mathrm{i}, \mathrm{j})} / \mathrm{N}_{\mathrm{i}}\right)$ and multivariate GLM analyses we found that: $(\mathrm{a})$ 
while BPIS composed a mean of $8.9 \%$ of individuals activity budgets, it comprised only $1.7 \%$ of behavioural transitions; (b) there were no species differences in probabilities of transitions including BPIS; (c) females presented more transitions to head-twirl than males; (d) individuals on the island exhibited less transition to sexual display and ingestion of faeces than the individuals in enclosures. These results indicate that BPIS disrupts optimal behaviour patterns, particularly vigilance and exploration of the environment. It also revealed differences between sexes and animals kept in different enclosures, suggesting that this analysis is a useful tool to complement animal welfare state evaluations. This research was approved by the Brazilian agency for wildlife protection (ICMBio permit numbers: SISBIO 38855-2, 42073-1) and complied with the International Primatological Society Guidelines for the Use of Nonhuman Primates in Research.

\title{
Are Tonkean Macaques Able to Take the Visual Perspective of Their Conspecifics?
}

\author{
Charlotte Canteloup ${ }^{a, b}$, Emilie Piraux $^{a}$, Hélène Meunier ${ }^{a, b}$ \\ ${ }^{a}$ Centre de Primatologie de I'Université de Strasbourg, Niederhausbergen, and \\ bLaboratoire de Neurosciences Cognitives et Adaptatives, UMR 7364, Strasbourg, France \\ E-Mail: charlotte.canteloup@gmail.com
}

Human beings had been considered to be the only species capable of Theory of Mind until about ten years ago, when studies first reported that chimpanzees were able to attribute mental states, such as seeing, knowing, intending and inferring, to others. One component of Theory of Mind is the ability to perceive what others can and cannot see, i.e. visual perspective-taking. It is generally attributed to only great apes and humans. However, this ability has been little investigated in monkeys and is still debated. In the present study, we investigated whether Tonkean macaques, Macaca tonkeana, know what conspecifics do and do not see. To do this, we used a food competition paradigm originally developed in chimpanzees by Hare and colleagues. We tested the individuals in pairs, after recording the dominance relationship of each dyad. Eighteen dyads were tested in four different experimental conditions. In one condition, the subordinate subject had the choice between two pieces of food, one that was visible only to it and the other that was also visible to the dominant conspecific. In this paradigm, if the subordinate understands that the dominant cannot see both pieces of food because one is hidden from its view, it is predicted to pick the piece of food that is visible only to itself. In the three other conditions, we varied the temporal and visual access to food for dominant and subordinate to control that (i) the subordinate's choice is not a result of those made by the dominant and (ii) macaques do not have a preference for hidden food - independently of the experimental context. Our results, currently being analysed, will be presented and discussed in the light of the evolution of cognition. This research complied with the European Directive 2010/63/EU and was approved by the French Ethical Committee CREMEAS (Number of agreement for conducting experiments on primates: $\mathrm{AL} / 46 / 53 / 02 / 13)$. 


\title{
Does the Presentation Format Affect Learning Speed in Visual Discrimination Tasks? A Study on Tufted Capuchin Monkeys (Sapajus spp.)
}

Paola Carduccia, ${ }^{a}$, Cinzia Trapanese ${ }^{a}$, Daniel Hanus ${ }^{c}$, Valentina Truppa ${ }^{a}$

a Unit of Cognitive Primatology and Primate Centre, Institute of Cognitive Sciences and Technologies, National Research Council (CNR), and bepartment of Biology, University of Rome Tor Vergata, Rome, Italy; ' Department of Developmental and Comparative Psychology, Max Planck Institute for Evolutionary Anthropology, Leipzig, Germany E-Mail: carducci.paol@gmail.com

Diurnal primate species are frequently studied in the context of the visual cognition domain since they mostly rely on sight to gather information from the environment. Experimental paradigms used to study visual behaviour in humans and non-human species are based on discrimination tasks involving different types of stimuli and procedures for stimuli presentation, thus stressing the necessity to compare data obtained with different methodological approaches. In this study, we assessed if the type of presentation format affected capuchin monkeys' ability to solve a size discrimination problem. Capuchins' ability to generalise knowledge across different presentation formats was also evaluated. Eight adult tufted capuchin monkeys (Sapajus spp.) were trained to select the larger of two stimuli of the same shape and different size by using pairs of items. Experiment 1 involved two experimental conditions: Food condition where the capuchin monkeys had to discriminate between two food items; and Image condition where they had to discriminate between two images. Experiment 2 involved only one experimental condition $(\mathrm{Ob}-$ ject condition) in which capuchin monkeys had to discriminate between two objects. Results showed that monkeys reached the learning criterion faster with food stimuli compared to both images and objects. They also consistently required fewer trials to reach the criterion with objects than with images. Finally, capuchins did not immediately transfer the solution of the problem acquired in one task condition to the other conditions. Overall, these findings indicate that the same problem presented with different - although functionally equivalent - methods led to consistent variations in learning behaviour. This suggests that one should be very cautious when comparing cognitive performances between different presentation formats or experimental paradigms and it encourages further research on the mechanisms underlying such methodologydependent differences. Our research complied with the European Directive 2010/63/EU for the Use of Nonhuman Primates in Research. The research protocol was approved by the Italian Health Ministry (Central Direction for Veterinary Service, approvals No. 11/2011-C and No. 132/2014-C).

\section{New Insights into Hair Cortisol Analysis for Long-Term Stress Monitoring in Chimpanzees (Pan troglodytes)}

\author{
Esther H.D. Carlitz ${ }^{a, b}$, Clemens Kirschbaum ${ }^{b}$, Robert Miller ${ }^{b}$, Joshua Rukunduc , Titus Mukunguc, \\ Carel P. van Schaik ${ }^{a}$ \\ ${ }^{a}$ Anthropological Institute and Museum, University of Zurich, Zurich, Switzerland; \\ bepartment of Biopsychology, Technische Universität Dresden, Dresden, Germany; \\ 'Chimpanzee Sanctuary and Wildlife Conservation Trust, Entebbe, Uganda \\ E-Mail: ecarlitz@janegoodall.ch
}

Hair cortisol concentrations (HCC) are increasingly recognized as useful objective measures of long-term stress in mammals, but there is still a large gap in knowledge about the practi$\mathrm{cal}$ significance and the underlying mechanisms of confounding factors. Two problems stand out:

6th European Federation for Primatology Meeting
Folia Primatol 2015;86:235-386 DOI: $10.1159 / 000435825$ 
the effect of body region, and cortisol decrease along the hair shaft (waning effect). In addition, the reliability of the HCC measure for inter-individual comparisons of stress levels is not yet known. Significant differences between certain body regions (chest $>$ shoulder blade $>$ forearm $>$ back) were found using hair from semi-wild and zoo-living chimpanzees. However, strong correlations between regions $(r \geq 0.6, \mathrm{p}<0.001)$ and results from a factor analysis suggest that the HCC values of all body regions mainly reflect one process and provide a similar biological signal. Comparisons with thermal images further suggest that intra-individual differences in skin blood flow may be the underlying mechanism of the body region effect. Concerning the waning effect, HCC along the hair shaft showed a strong, systematic decline towards distal segments in semiwild-living chimpanzees but was numerically negligible in zoo-living chimpanzees. Correlations between HCC and the amount of rain that hair was exposed to, suggest that other factors than rain are more likely to cause the strong waning effect in semi-wild-living chimpanzees. Finally, we found that HCC levels were biologically meaningful at individual levels: our results revealed a strong correlation with large effect size between HCC and animal keeper-ranked stress levels for 38 chimpanzees $\left(p r_{s}=0.6, \mathrm{p}<0.001\right)$. In conclusion, HCC levels in chimpanzees are biologically meaningful if body region and waning effect are considered.

\section{The Semantics of Titi Monkey Alarm Calls}

Cristiane Cäsar ${ }^{a, b}$, Richard W. Byrne ${ }^{a}$, Klaus Zuberbühler $^{a, c}$

aSchool of Psychology and Neuroscience, University of St Andrews, St Andrews, UK:

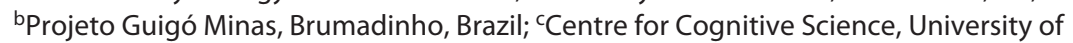
Neuchâtel, Neuchâtel, Switzerland

E-Mail: criscasar@gmail.com

Primates communicate about objects and events in their environment in ways that allow recipients to draw inferences about the nature of the event experienced by the signaller. Here, we present the results of a naturalistic and experimental study with several habituated groups of a Neotropical primate species, the black-fronted titi monkey (Callicebus nigrifrons), living in the Caraça Reserve, Minas Gerais, Brazil. The aim was to determine the exact mechanisms of alarm calling behaviour, with an emphasis on production and comprehension. Results showed that, when detecting predator species, C. nigrifrons produces sequences that initially contain two types of brief, high-pitched calls with distinct frequency contours. Playback experiments showed that some of these sequences are meaningful to conspecific receivers, by indicating the general predator class and location of the threat. The presence of different call types within the terrestrial threats suggested that additional information may be encoded by acoustic and compositional differences. Analyses of call order and number of calls per sequence suggested that callers may be able to convey information on both predator type and location. The black-fronted titi monkey's vocal system thus provides a further example of zoo-syntax, in which acoustically fixed units of a vocal repertoire are combined into higher order sequences that are meaningful to recipients. This research complied with the International Primatological Society (IPS) Guidelines for the Use of Nonhuman Primates in Research. 


\title{
Enriching Ring-Tailed Lemurs: Evaluating the Effect of Three Types of Enrichments on Animal Behaviour
}

\author{
Marta Caselli ${ }^{a}$, Patrizia Messeri ${ }^{a}$, Francesco Dessì-Fulgheri ${ }^{a}$, Francesca Bandoli ${ }^{b}$ \\ aDipartimento di Biologia Evoluzionistica Università degli Studi di Firenze, Florence, and \\ ${ }^{\mathrm{b}}$ Giardino Zoologico di Pistoia, Pistoia, Italy \\ E-Mail: marta.caselli@stud.unifi.it
}

Enrichment is a management tool used to provide different stimuli and promote animal welfare encouraging species-specific behaviour. This project aimed to evaluate the effect of three types of enrichments on a captive group of six ring-tailed lemurs (Lemur catta) hosted at Giardino Zoologico di Pistoia through behavioural analysis. Data were collected over a three-month period using a within-subjects experimental design consisting of five conditions: baseline (BL); food-based enrichment (E1); physical enrichment (E2); auditory enrichment (E3); no enrichment provided (NE). Each condition was randomly repeated seven times. Six 30-min observation sessions were undertaken daily and data were collected via two sampling techniques: (i) 2-min focal animal sampling per individual per observation period; (ii) instantaneous scan sampling of the whole group with 5-min intervals. A total of $107 \mathrm{~h}$ of observation were conducted. Data were analysed using Wilcoxon matched-pairs signed-rank test with significance level set at $\mathrm{p}<0.05$ and Spearman rank correlation coefficient. Results showed that enrichments significantly decreased the frequency of resting behaviours and raised activity levels, increasing the frequency of vigilance, locomotion, olfactory and scent-marking behaviours. Their effectiveness did not decline as repetition number increased suggesting that providing enrichments on a random schedule is useful to avoid habituation. These findings highlight the key role played by enrichments in stimulating positive behavioural changes in a captive environment. The study was performed in accordance with the European Directive 2010/63/EU.

\section{Behavioural and Physiological Effects of Housing Type on Laboratory Housed Female Rhesus Macaques (Macaca mulatta)}

Lauren Cassidy $^{a}$, Stuart Semple ${ }^{a}$, Darcy Hannibal ${ }^{b}$, Brenda McCowan ${ }^{b, c}$

aCentre for Research in Evolutionary and Environmental Anthropology, University of Roehampton, London, UK; ${ }^{b}$ California National Primate Research Center, University of California, and 'Department of Population Health Reproduction, School of Veterinary Medicine, University of California, Davis, Calif., USA

E-Mail: cassidyl@roehampton.ac.uk

Due to the despotic nature of rhesus macaques (Macaca mulatta) and their frequent use in biomedical research, it is important to understand the impact that standard housing practices can have on individuals of this species in order to improve practices and maximize laboratory primate well-being. This study takes advantage of existing pair housing conditions that occur at the California National Primate Research Center (CNPRC): (1) intermittent pair housing, which involves the daily separation of pair-mates often due to suspected feeding aggression, and (2) continuous pair housing, where pair-mates are rarely separated. Study subjects were 24 adult female rhesus macaques, 12 initially intermittently housed and 12 initially continuously housed. These pairs were studied in their initial pairing condition for two weeks, after which their pairing condition was experimentally changed (e.g. intermittent to continuous) and they were studied for an additional three weeks. Behavioural observations recorded affiliative behaviour, status signals, aggression, social compatibility behaviour and behaviours indicative of anxiety or stress. Additionally, each animal was non-invasively physiologically sampled for afternoon urine and these sam- 
ples analysed for free cortisol (a biomarker of stress). Behavioural and physiological measures were compared between intermittent and continuous contact pairing conditions. Change in behaviour over time after the initial pairing condition is modified was also explored. I will present the results of this study and explore their implications in this poster. This study complies with the European Directive 2010/63/EU and the International Primatological Society Guidelines for the Use of Nonhuman Primates in Research. Approvals for this study have been obtained from the CNPRC Research Advisory Board, University of California Davis Institutional Animal Care and Use Committee and the University of Roehampton Ethics Committee.

\title{
Preliminary Data on the Population Density and Conservation Status of Two Endangered Primate Species Inhabiting a Protected Area on the West Coast of Ecuador
}

\author{
Laura Cervera ${ }^{a, b}$, Diego Tirira ${ }^{c, d}$, Giuseppe Donati ${ }^{b}$ \\ aDepartamento Central de Investigación, Universidad laica Eloy Alfaro de Manabí, \\ Manta, Ecuador; ${ }^{b}$ Oxford Brookes University, Oxford, UK; ' ${ }^{c}$ Museo de Zoología, Pontificia \\ Universidad Católica del Ecuador, and ' Fundación Mamíferos y Conservación, Conocoto, \\ Quito, Ecuador \\ E-Mail: laura.cervera24@gmail.com
}

Howler (Alouatta spp.) and capuchin monkeys (Cebus spp.) are among the most studied Neotropical primates and both taxa are well known for their ability to survive in highly disturbed habitats. However, there is a lack of information on the Ecuadorian populations of these two genera which occur there in some of the most threatened South American ecosystems, including the coastal region located in the Tumbes-Chocó-Magdalena hotspot. This study aimed to evaluate the effects of anthropogenic disturbance and habitat characteristics on the population density of the Vulnerable mantled howler monkey (Alouatta palliata aequatorialis) and the Critically Endangered white-fronted capuchin monkey (Cebus albifrons aequatorialis) in the Marine and Coastal Wildlife Refuge of Pacoche in western Ecuador. Although protected, illegal activities inside the area include slash-and-burn agriculture, natural resource extraction and small-scale hunting. We distributed eighteen transects of $1 \mathrm{~km}$ each in the protected area in order to determine population density in the reserve and assess the impact of anthropogenic disturbance. Each transect was walked 5 times, totalling over $90 \mathrm{~km}$ of surveying effort, yet we did not detect capuchins in the area while walking the transects. White-fronted capuchin monkeys were observed on three occasions, confirming their presence in the area. We estimated mantled howler monkey density to be $20.44 \mathrm{ind} / \mathrm{km}^{2}$, and the average group size was $9.28 \pm$ SD 4.30 individuals. Results suggest that probability of detection varied with group size and A. palliata aequatorialis density followed a half normal distribution correlated with distance to the road. Canopy cover was also associated with probability of detection, which highlights the importance of habitat connectivity. The very low detection of white-fronted capuchins in the area indicates the need for immediate conservation actions for the primates of the Ecuadorian coastal region. 


\title{
Gibbon Sleeping Sites: Selection, Function, Variation and Impacts on Activity Budgets
}

\author{
Susan M. Cheyne ${ }^{a, b}$, Fan Peng-Feic
}

aOrang-utan Tropical Peatland Project, Palangka Raya, Indonesia; b' Wildlife Conservation Research Unit, Department of Zoology, Oxford University, Oxford, UK; 'Dali University, Dali, Yunnan, People's Republic of China

E-Mail: scheyne@outrop.com

We carried out a comprehensive review of knowledge about gibbon sleeping sites and sleeping behaviour involving 15 studies and 12 species. We assessed the trade-offs between a variety of predictors and variables influencing gibbon sleeping site choice. Physical aspects assessed were tree comfort, stability, accessibility (for predation risk and competitor avoidance) and parasite avoidance. Ranging variables included location of sleeping tree in relation to next day travel routes, daily path length (DPL) and seasonality. The location of sleeping tree within the home range was also assessed as this could influence the extent of territorial defence. Ecological variables assessed were variation with altitude, habitat type, intensity of anthropogenic disturbance and habitat fragmentation. Preliminary results indicate very little variation in terms of times entering and leaving sleeping trees, location of these trees and choices of stability and predator avoidance irrespective of the wide variation in study site altitude and habitat type. Further information and the implications of this apparent standardisation of sleeping behaviour across species will be discussed. We note that identifying sleeping sites for gibbons is very useful for assisting in their monitoring and, therefore, conservation management. For species for which we have no sleeping site information, e.g. Hainan gibbon (Nomascus hainanensis), identifying the location of such sites should be a priority.

\section{Contest Hooting Behaviour as a Window into Social Relationships in Wild Bonobos}

\author{
Zanna Clay ${ }^{a}$, Gottfried Hohmann ${ }^{b}$, Klaus Zuberbuhler ${ }^{a, c}$ \\ anstitute of Biology, University of Neuchâtel, Neuchâtel, Switzerland; ${ }^{\mathrm{b}}$ Department of \\ Primatology, Max Planck Institute for Evolutionary Anthropology, Leipzig, Germany; \\ 'School of Psychology and Neuroscience, University of St Andrews, St Andrews, UK \\ E-Mail: zannaclay@gmail.com
}

Vocalisations represent an important tool for animals to navigate their social and physical landscapes. They also offer a useful window to explore social relationships and underlying social awareness. In bonobos (Pan paniscus), males produce a conspicuous display vocalisation, known as the 'contest hoot', which they typically direct towards a specific target. Our previous research in captivity suggested that males use this behaviour to test social relationships with others, with the main goal being to provoke a social reaction in their target (Genty et al., 2014). Here, we examined contest hooting among wild bonobos in order to investigate underlying patterns of intraand inter-sexual dominance relationships and its role as a tool for social exploration by males. We observed male $(n=7)$ contest hooting behaviour in the Lui Kotale bonobo community, DR Congo. Males directed contest hoots at specific targets, and the dominance relationship of the hooter and the target reliably predicted the target's social response. While all males targeted both sexes, each male had a preferred social target that was most likely to be the male of adjacent dominance rank. Contest hooting also frequently provoked audience barking and third-party interventions by high-ranked audience members. There was also a significant effect of audience, with contest hoots more likely to occur after fusions, where group size increased. Overall, results suggest that contest hoots are a ritualised vocal behaviour that males use to flexibly test and explore their social dominance relationships within a public arena. Evidence of third-party interventions 
suggests that beyond dyadic relationships, contest hoots also enable males to explore broader alliance relationships within their group. Research complied with the International Primatological Society Guidelines for the Use of Nonhuman Primates in Research and adhered to the requirements and guidelines of the ICCN and to legal requirements of the DR Congo.

\section{The Mediating Role and the Emphatic Impact of Females in Conflict Management: The Investigation of Consolation Dynamics in Pre-School Children}

Giada Cordoni ${ }^{a}$, Elisabetta Palagi ${ }^{a, b}$

aNatural History Museum, University of Pisa, Calci, Pisa, and bInstitute of Cognitive Sciences and Technologies, Unit of Cognitive Primatology and Primate Centre, CNR, Rome, Italy

E-Mail: cordoni@vademecos.eu

The occurrence of 'consolatory' behaviours, directed towards distressed individuals or victims of aggression, is a widespread phenomenon throughout the animal kingdom, having been documented in primates, carnivores, proboscideans, rodents and corvids. The capacity to understand and share the emotional states of others, labelled as empathy, is the basis of 'consolatory' behaviours. In humans, empathy is involved in numerous social arenas, such as, conflict management, parental care, love affairs and compassion. Recently, neurobiological research revealed significant qualitative and quantitative gender differences in affective and cognitive forms of empathy and in the influence of emotions in the decision making process. Indeed, females appear to be more empathic and more competent in understanding others' internal states. Moreover, females show a higher propensity to compromise and mediate compared to males. We carried out an ethological study on 137 pre-school children, ranging from 3 to 5 years old, hosted at the kindergarten 'Florinda' (Viareggio, Italy). We assessed the occurrence of consolation after an aggressive event in all age-classes under study (3 years old - mean group TCT $46.99 \% \pm 12.36 \mathrm{SE}, 4$ years old - mean group TCT $52.64 \% \pm 13.60 \mathrm{SE}$, 5 years old - mean group TCT $62.75 \% \pm 16.64 \mathrm{SE}$ ). The consolatory behaviour is used to evaluate if a difference in empathic and mediation abilities exist between males and females. Moreover, since some studies suggested that as children get older their empathic propensity acquire more cognitive elements, we investigated if gender difference in this propensity is also linked to age by delineating possible ontogenetic trajectories of this capacity in human primates.

\section{Rural Men and Women's Perceptions of Livelihood Risks in Relation to the Cantanhez Protected Forest National Park, Guinea-Bissau (West Africa)}

Susana G. Costa ${ }^{a, b}$, Catarina Casanova ${ }^{b}$, Phyllis C. Lee ${ }^{a}$

aSchool of Natural Sciences, Division of Psychology, University of Stirling, Stirling, UK;

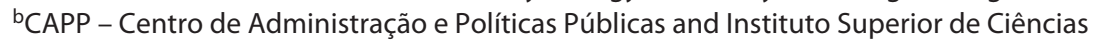

Sociais e Políticas da Universidade de Lisboa, Lisboa, Portugal

E-Mail: susanagkosta@gmail.com

We compare locals' perceptions of the major cognitively-based livelihood risks which were associated with living in the Cantanhez Forest National Park (CFNP), south Guinea-Bissau. This study was one part of a Western chimpanzee (Pan troglodytes verus) conservation project. There are 9 other primate species in the CFNP: Guinea baboon (Papio papio), red Western colobus (Procolobus badius temminckii), putty-nosed monkey (Cercopithecus nictitans), lesser spot-nosed monkey (Cercopithecus petaurista), green monkey (Chlorocebus sabaeus), black and white Western colobus (Colobus polykomus), Campbell's monkey (Cercopithecus campbelli), Sooty mangabey 
(Cercocebus atys), and the bushbaby (Galago senegalensis). We focus on the stated perceptions of the benefits that CFNP could bring and contrast these with the imposed livelihood constraints revealed by people during survey questionnaires $(n=257)$, in-depth interviews $(n=47)$ and focus groups ( $n=5$ ). Three specific issues were considered: (i) People living in CFNP are dependent on subsistence farming activities in an impoverished and changing ecosystem which exacerbates feelings of uncertainty in relation to their future; (ii) Some farming activities are now constrained, due to policy restrictions imposed by the CFNP's authorities; (iii) The establishment of the CFNP has not been associated with any form of compensation for lost livelihood activities. Data suggested that the local inhabitants were dependent on subsistence agriculture occurring within the CFNP, and were generally negative in their statements of potential benefits resulting from the park. Some men took a longer term perspective, emphasizing the potential revenue generation which might result from future ecotourism, while women could see no short- or long-term advantages. The top livelihood risks was identified as famine for both men and women, which was perceived as resulting from the new constraints imposed on livelihoods by the establishment of the CFNP.

\section{Cryptic Species and Species Concept in Palaeontology Sébastien Couette \\ Ecole Pratique des Hautes Etudes \& UMR uB/CNRS 6282 'Biogéosciences', Dijon, France E-Mail: sebastien.couette@u-bourgogne.fr}

Cryptic species and species complexes are great challenges for biologists. New molecular methods lead to the discovery of species complexes, the identification of new clades and, even, the multiplication of species. Morphologists and, especially, paleo-primatologists have a different approach to the species concept, based mainly on the dissimilarities observed in the specimens. This approach can be, in certain circumstances, very conservative, especially when morphological variations between species are low. Thus, cryptic species have been completely disregarded by paleo-primatologists. Adapis parisiensis, a primate species from the Eocene of Europe, is one of the most studied fossil species. Historically, many fossils from the Eocene were attributed to this species. Some reviews of the fossil material and new discoveries increased the diversity of the genus. However, the variation described for several morphological traits is intriguing and still badly understood. In this study we investigated the morphological variation of this genus with the idea of cryptic species. We also analysed the different inferences of ecology and behaviour within the species in order to interpret them in term of species concepts. Based on the morphology of cranial, dental and postcranial remains, and of their interpretation, we suspect the presence of different species in what has been considered for years as a single species.

\section{Suffixation in Non-Human Primates: Meaningful Sound Combinations in Free-Ranging Guenons}

Camille Coye $^{a, b}$, Klaus Zuberbühler $^{b, c}$, Alban Lemasson ${ }^{a, d}$

aLaboratoire EthoS, Université de Rennes 1, U.M.R 6552-C.N.R.S, Station Biologique de Paimpont, Paimpont, France; bSchool of Psychology and Neuroscience, University of St Andrews, St Andrews, UK; Institut de Biologie, Université de Neuchâtel, Neuchâtel, Switzerland; ' Institut Universitaire de France, Paris, France E-Mail: camille.coye@univ-rennes1.fr

Compared to humans, non-human primates have very little control over their vocal production. Nonetheless, some species produce various call combinations, which may partially offset this lack of flexibility. In previous work, we have described context-dependent call combinations in 
male alarm calls and female social calls of two sympatric species of African forest guenons, Campbell's monkeys (Cercopithecus campbelli) and Diana monkeys (C. diana). In particular, male Campbell's monkeys give one alarm call type ('Krak') to leopards and a suffixed version of the same call ('Krak-oo') to unspecific danger. Similarly, female Diana monkeys give four social calls emitted alone or merged into six combinations; in combinations, the initial call conveys information about social context, while the final call conveys information about caller identity. We performed playback experiments using natural and artificially combined calls for both male Campbell's and female Diana monkey calls, using wild Diana monkeys as subjects in both experiments. Responses indicated that monkeys attended to the way calls were combined regardless of contextual origin. We conclude that non-human primates are able to produce meaningful call combinations in both predatory and social contexts, which generates interesting hypotheses about the nature and evolution of sound combination in the evolution of primate communication. This study was conducted in accordance with the current International Primatological Society Guidelines and has been approved by the University of St Andrews (School of Psychology) ethics committee.

\title{
Spatial Ecology and Behavioural Correlates of Crop-Raiding of Baboons (Papio ursinus) in a Human-Modified Habitat
}

Marianna Cravero ${ }^{a}$, Selena Esposito ${ }^{a}$, Fabien Genin ${ }^{b}$, Judith Masters ${ }^{b}$, Marco Gamba $^{a}$

aUniversità di Torino, Turin, Italy; bUniversity of Fort Hare, Alice, South Africa

E-Mail: mariannacravero@yahoo.it

The transformation of landscapes from predominantly wild to predominantly anthropogenic has led to high levels of competition between humans and wild animals for space and resources. Anthropogenic habitat alteration can have a dramatic effect on the spatial distribution and ranging patterns of primates, and can induce them to incorporate crop-raiding among their foraging strategies. We studied the spatial ecology and the crop-raiding behaviour of two freeliving troops of chacma baboons (Papio ursinus) in Hogsback, South Africa. Both troops have free access to natural and human-modified habitat. We collected data from September to November 2013. We imported GPS data points for both troops in ArcGIS 9.2, in order to quantify their home range size, habitat use and variations in travel speed. Furthermore, we used behavioural observations to identify the key parameters of crop-raiding and to investigate the impact of crop-raiding on the activity budget. The two troops have relatively small home ranges, a high density and a low travel speed. Both troops spent most of their time in the human-modified habitat and did not use their home range uniformly. Duration of the crop-raiding events was higher in the morning and was not influenced by human or dog presence. The number of individuals engaged in crop-raiding was independent of troop size. They spent less time moving and foraged more during the raids when compared to the time spent moving and foraging outside private properties. Spatial and behavioural ecology of the baboons living in a human-modified habitat differs from that of conspecifics whose ranging is limited to natural habitats. This research complied with the International Primatological Society (IPS) Guidelines for the Use of Nonhuman Primates in Research.

\author{
How Intentional Are Chimpanzee Vocalizations? \\ Catherine Crockford ${ }^{a}$, Roman Wittig ${ }^{a}$, Klaus Zuberbühler ${ }^{b}$ \\ aMax Planck Institute for Evolutionary Anthropology, Leipzig, Germany; bUniversity of \\ Neuchâtel, Neuchâtel, Switzerland \\ E-Mail: crockford@eva.mpg.de
}

A unique capacity in human communication is that speech is produced with the intention to influence the thoughts or actions of listeners. A key question in research focusing on the evolution of language is whether other animal vocalisations are also produced with the intention to 
change another's behaviour (1st order intentionality sensu Dennett 1983) or to change another's mind (2nd order intentionality sensu Dennett 1983). There is considerable evidence that chimpanzees produce gestures with the intention to change another's behaviour. However, it is only recently that evidence suggests that this is also the case for chimpanzee vocal production. Here, we collate new and existing evidence of at least 1st order intentional vocal production in chimpanzees. In addition, we discuss how much chimpanzee vocal production may be under voluntary control and produced with the intention to change another's knowledge states. We will also discuss the implications of this work for intentional vocal production in other primates. This research complies with the European Directive 2010/63/EU and the International Primatological Society Guidelines for the Use of Nonhuman Primates in Research.

\title{
Using a Dental Ecology Approach to Assess the Ecology and Behaviour of Wild Otolemur crassicaudatus in South Africa: Results from Dental Health and Faecal Analyses
}

\author{
Frank P. Cuozzo ${ }^{a}$, Michelle L. Sauther ${ }^{b}$, Alycia E.A. Hall ${ }^{a}$, Sydney D. Johnson ${ }^{a}$, Adrian Tordiffe, \\ Krista D. Fish ${ }^{d}$ \\ aDepartment of Anthropology, University of North Dakota, Grand Forks, N.Dak., \\ ${ }^{\mathrm{b}}$ Department of Anthropology, University of Colorado, Boulder, Colo., USA; ${ }^{\mathrm{C}}$ National \\ Zoological Gardens of South Africa, Pretoria, South Africa; ${ }^{\mathrm{d} D e p a r t m e n t ~ o f ~ A n t h r o p o l o g y, ~}$ \\ Colorado College, Colorado Springs, Colo., USA \\ E-Mail: frank.cuozzo@email.und.edu
}

The galagos of continental Africa are among the world's least studied primates. In 2013 we began a comprehensive study of the ecology, biology and behaviour of two sympatric galago species at the Lajuma Research Centre, South Africa. Across two field seasons (2013 and 2014) we safely captured 24 adult thick-tailed bushbabies (Otolemur crassicaudatus). Dental health analyses indicate a high frequency of broken maxillary canines, each associated with decay and apical abscesses that present as open wounds on the snout (92\%). There is no sex difference in the frequency of this pathology (female $93 \%, \mathrm{n}=14$; male $90 \%, \mathrm{n}=10$ ). Previous wild studies suggest that this species uses gums as a winter food source, often requiring an individual to gouge tree bark to access the gums. We hypothesized that the source of the frequent maxillary canine damage in this population is the gouging of bark to access gums. Among the teeth in this species, the maxillary canines are short and stout and likely the only teeth capable of such food acquisition. To test this, in 2014 we collected faecal samples from 11 live traps across 5 days at Lajuma to assess food remains, and thus feeding ecology. Faecal samples were not identified to individual. Among the foods present in these faecal samples were seeds, bark and most surprising, frequent ant exoskeletons (Family Formicidae). The presence of bark in the Lajuma faecal samples indicates gum feeding and suggests that use of the stout maxillary canines to gouge tree limbs in order to access gums leads to the high frequency of canine damage in this population. The high frequency of ant remains in these faecal samples suggests that during the nutritionally challenging winter season, this population of $>1 \mathrm{~kg}$ strepsirrhine primates utilizes social insects as a common food source. These data illustrate how a dental ecology approach can provide an understanding of the ecology and behaviour of a lesser-studied primate species. 


\title{
Determinants of Variation in Orangutans' Cognitive Performance: I. Effects of Background and Housing Conditions
}

\author{
L.A. Damerius ${ }^{a}$, S.I.F. Forss' ${ }^{a}$, Z.K. Kosonen ${ }^{a}$, J.M. Burkart ${ }^{a}$, D.B. Haun ${ }^{b}$, K. Liebal', J. Call $^{\text {, }}$, \\ B.M. Galdikas ${ }^{e}$, C.P. van Schaik ${ }^{a}$ \\ ${ }^{a}$ Anthropological Institute and Museum, University of Zurich, Zurich, Switzerland;

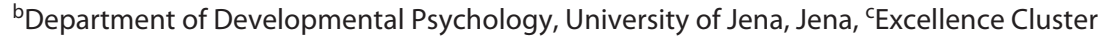 \\ 'Languages of Emotion', Freie Universität Berlin, Berlin, and dMax Planck Institute for

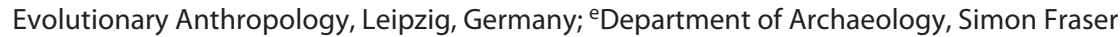 \\ University, Burnaby, B.C., Canada \\ E-Mail: laura.damerius@uzh.ch
}

Orangutans in the wild and captivity are known to be proficient problem solvers, and it is widely assumed that such an ability is adaptive in their challenging environment. The cultural intelligence hypothesis predicts a major effect of social-cultural and housing conditions on the development of their cognitive abilities. In this study we examine whether Bornean and Sumatran orangutans (Pongo pygmaeus and Pongo abelii), housed at nine different European zoos and four different sanctuaries across Central Kalimantan and Sumatra in Indonesia, vary in their problemsolving abilities. We compared the cognitive performance of up to 102 individuals on three different standardized tasks: detour reaching, the tube-trap task and the visible honey-trap task, in order to test for inhibitory control, learning abilities and tool use, and thus general problemsolving strategies. Preliminary results show great variability in cognitive performance across individuals and settings, as well as significant differences between zoo-living and sanctuary-housed Bornean and Sumatran orangutans. These effects of background and rearing conditions, summarized in various derived measures, are consistent with the cultural intelligence hypothesis. All experiments fully comply with the ethical guidelines of each study facility and the European Directive 2010/63/EU and are approved by the Ethics Committee of the University of Zurich in Switzerland and the Indonesian Ministry of Research and Technology.

\section{Art Therapy in Nonhuman Primates? Graphic Production as a Mechanism for Sensory Enrichment and Enhancement of Welfare in Chimpanzees (Pan troglodytes)}

Daniela D'Amore ${ }^{a}$, Yulán Úbeda $a^{a, b}$, Sandra Ballesta ${ }^{a}$, Miquel Llorente $^{a, c}$

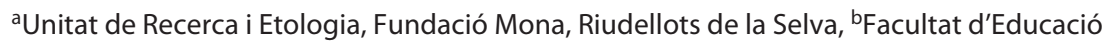
i Psicologia, Universitat de Girona, Girona, and 'Institut Català de Paleoecologia Humana i Evolució Social (IPHES), Tarragona, Spain

E-Mail: yulanubeda@hotmail.com

The aim of this research is to verify the effect of graphic production on the behaviour of two groups of sanctuary-housed chimpanzees. For this purpose, a painting session was given to the subjects of the study as a sensorial and cognitive enrichment during a predetermined period divided into 10 sessions for each of the eight subjects. Three subjects, who were in the process of re-socialization, were considered as an experimental group, and the other five belonged to the control group. Each session contained: pre-test phase, treatment phase (painting session) and post-test phase. All behaviours were video recorded for each session, and data were coded using a complete ethogram of 116 behaviours, assembled into 20 behavioural categories. From these data we also calculated two welfare indices (Llorente et al., 2014): Behavioural Competence Index (BCI), and Behavioural Diversity Index (BDI). Some significant behavioural changes were found during the treatment phase, some of them were: the increment of manipulative behaviours, the decrease in inactive behaviours and, also, the improvement of both welfare indices (BCI \& BDI). 
The increase of positive behavioural categories during the treatment phase indicates an improvement of the chimpanzees' well-being and welfare. There were also some differences between groups: the experimental group, due to it being in the process of re-socialization, showed more self-directed behaviours and lower levels of Behavioural Competence Index (BCI). It can be concluded that the art therapy treatment, as conducted during this research, had a positive effect on the welfare of the individuals in this study. This research complied with the European Directive 2010/63/EU and the International Primatological Society (IPS) Guidelines for the Use of Nonhuman Primates in Research.

\section{Aesthetics in Primate Paints}

Daniela D'Amore ${ }^{a, d}$, Yulán Úbeda ${ }^{a, e}$, Sandra Ballesta ${ }^{a}$, Antonella Minellid ${ }^{d}$ Miquel Llorente ${ }^{a-c}$

aUnitat de Recerca i Etologia, Fundació Mona, Riudellots de la Selva, ' IPHES, Institut Català de Paleoecologia Humana i Evolució Social, and 'Àrea de Prehistòria, Universitat Rovira i Virgili (URV), Tarragona, Spain; d Dipartimento di Scienze Umanistiche, Sociali e della Formazione, Università degli Studi del Molise, Campobasso, Italy; ${ }^{\mathrm{e} F a c u l t a t}$ d'Educació i Psicologia, Universitat de Girona, Girona, Spain

E-Mail: mllorente@fundacionmona.org

The aim of this research is verify whether there is an aesthetic sense in non-human primates that can be expressed using graphic productions, which characteristics are, more or less, similar to those of human productions. To do this, we used paintings made by eight chimpanzees during art therapy sessions at Fundació Mona Sanctuary (Girona, Spain). A selection of 30 paintings was evaluated by 60 volunteers. We designed an Aesthetics Primate Paint Questionnaire (APPQ), composed of 32 trait-descriptive items (with a rating scale from 1 to 7 ) regarding features such as colours, figures, direction of the brush strokes and the use of space in each painting. In the instructions for filling out the questionnaire, we recommended avoiding consultations with other analysts. We used a Principal Component Analysis to identify the factor structure of the questionnaire. We calculated the ICC (Intraclass Correlation Coefficient) to assess the reliability of the evaluators. From a first analysis, the results indicated a high level of reliability among raters and, secondly, a clear factor structure composed of eight factors. The factors identified were related to 'colours', 'use of space', 'symmetry', or 'aesthetic', among others. So, we highlight that these results are the first quantitative evidence of aesthetic sense in chimpanzees, but we also emphasize that this is a preliminary work, that concerns only a small sample of chimpanzees housed in a sanctuary. We consider that in future this type of study should be replicated and extended to other primates housed in recovery centres with large sample sizes. In this way, the model could be very important in order to increase the ecological validity of further studies to obtain information on the aesthetic sense of primates and the implications this entails for the management and welfare of these animals rehabilitated in sanctuaries. This research complied with the European Directive 2010/63/EU and the IPS Guidelines for the Use of Nonhuman Primates in Research. 


\title{
Overview of the Jane Goodall Institute's Scientific Research and Approach to Conservation
}

\author{
Daniela de Donno ${ }^{a}$, Debby Cox ${ }^{b}$, Liliana Pacheco ${ }^{c}$, Lilian Pintea ${ }^{b}$, Shawn Sweeney ${ }^{b}$ \\ ${ }^{\mathrm{a}}$ The Jane Goodall Institute Italia, Rome, Italy; ${ }^{\mathrm{b}}$ The Jane Goodall Institute USA, \\ Vienna, Va., USA; 'The Jane Goodall Institute Spain/Senegal, Fouta Djallon, \\ Kédougou-Dindéfélo, Senegal \\ E-Mail: daniela.dedonno@janegoodall-italia.org
}

The Jane Goodall Institute (JGI) continues Dr Jane Goodall's pioneering research with wild chimpanzees, advances innovative conservation to protect forest ecosystems across the chimpanzee range and raises awareness about how everyday actions impact our planet. The Gombe Stream Research Centre (GSRC) is the longest ongoing field research site for great apes in the world. This year marks 55 years of continuous study on three communities of chimpanzees in a single population. With the long-term data collected at Gombe we have a more comprehensive assessment of chimpanzee ecology, and we can understand better the interactions among their communities, as well as between humans and chimpanzees. GSRC continues to provide a platform for scientists to advance research and understanding of chimpanzee behaviour which ultimately drives more effective conservation. Extending across the chimpanzee's range, including Tanzania, Uganda, D.R. Congo, Republic of Congo, Gabon and Senegal, JGI's efforts inform a variety of scientific disciplines, including ageing, human evolution, ethology, anthropology, conservation biology, disease transmission, population biology, forest and landscape ecology. Over the next 30 years, the JGI will endeavour to protect $85 \%$ of the remaining chimpanzees and their habitats. The JGI has recognized that protecting species and their habitats cannot be separated from the task of improving the human condition. Using a holistic approach, informed strategically by the use of Open Standards to plan and implement conservation actions has enabled JGI projects to make a positive impact on both the human and chimpanzee populations in these countries. This holistic approach includes caring for confiscated chimpanzees, honouring Dr Goodall's core value that all individuals matter. For example, JGI's Tchimpounga Chimpanzee Rehabilitation Centre in the Republic of Congo cares for more than 150 chimpanzees and has served as an important site for cognitive, behavioural, genetic and parasitological studies.

\section{Navigation Patterns in Relation to the Use of Feeding and Resting Sites in Black Howler Monkeys (Alouatta pigra): A Preliminary Study}

Miguel de Guinea Luengo ${ }^{a}$, Sarie Van Belle ${ }^{b}$, Julia Lehmann $a$, Alejandro Estrada ${ }^{c}$

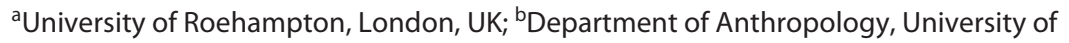
Texas, Austin, Tex., USA; ' Instituto de Biología, Universidad Nacional Autónoma de Mexico, Mexico, D.F., Mexico

E-Mail: mdeguinea@gmail.com

Animals have developed a variety of mechanisms to navigate within their home range in the most efficient manner, to reach feeding sites or avoid predators and competitors. Two systems have been proposed to explain navigation procedures: (1) a route-based map, which allows the forager to navigate through a series of travel routes using landmarks between feeding and resting sites, and (2) a coordinate-based map, by which the forager encodes and recalls the location of important sites as $\mathrm{x}$ and $\mathrm{y}$ coordinates, thereby providing a more flexible use of distances and directions. We test whether black howler monkeys (Alouatta pigra) use route-based or coordinatebased maps for navigation in Palenque National Park, Mexico. We observed two groups for six weeks each and recorded (i) directness of movements between feeding sites, (ii) the angle of approaching the site and (iii) the reuse of paths within their range. Our preliminary results suggest 
that directness decreased when paths were larger (up to $60 \%$ in paths above $110 \mathrm{~m}$ ). In addition, both groups approached the same feeding sites from different angles but tended to reuse the same routes. Taken altogether, the results suggest that black howler monkeys characterize their space mainly as route-based maps and that they navigate along a network of well-established interconnected paths. This research complied with the European Directive 2010/63/EU and the International Primatological Society (IPS) Guidelines for the Use of Nonhuman Primates in Research. It also complies with guidelines for animal studies of the government of Mexico (SEMARNAT) and the National Autonomous University of Mexico.

\title{
Growth Rates in Tonkean Macaques \\ Arianna De Marco ${ }^{a-c}$, Andrea Sanna $^{a}$, Melissa Messinese ${ }^{a}$, Bernard Thierry $^{d}$, Roberto Cozzolino $^{a}$ \\ ${ }^{a}$ Fondazione Ethoikos, Radicondoli, bParco Faunistico di Piano dell'Abatino, Poggio San Lorenzo, and Istituto di Scienze e Tecnologie della Cognizione, Consiglio Nazionale delle Ricerche, Rome, Italy; ${ }^{d}$ Institut Pluridisciplinaire Hubert Curien, Université de Strasbourg, Centre National de la Recherche Scientifique, Strasbourg, France \\ E-Mail: arianna.demarco@parcoabatino.org
}

The measurement of variations in body mass is necessary to understand the evolution of life history patterns and it provides information on the timing of sexual maturity and the development of sexual dimorphism. In this study, we collected longitudinal data on body mass from infancy to adulthood in a captive population of Tonkean macaques (Macaca tonkeana). We investigated the timing and magnitude of breaking points in the growth paths of 29 males and $20 \mathrm{fe}-$ males, and checked whether these breaking points could correspond to specific reproductive and morphological developmental events. We found roughly equivalent body mass in male and female Tonkean macaques until around the age of four, when males go through an adolescent growth spurt and females continue to grow at a constant rate. Males not only grow faster than females, but they also continue to grow for nearly one and half years after females have attained their full body mass. Growth rate differences account for approximately two-thirds of the body mass sexual dimorphism, and only the remaining third results from continued male growth beyond the age where full body mass is reached in females. We also discovered remarkable correspondences between the timing of testicular enlargement and the adolescent growth spurt in males, and between dental development and slowdown breaking points in both sexes. This research complied with the European Directive 2010/63/EU.

\section{Food Quantity, but Not Food Quality, Affects Temporal Preferences of Capuchin Monkeys (Sapajus spp.) in a Delay Choice Task}

\author{
Francesca De Petrillo ${ }^{a, b}$, Emanuele Gori ${ }^{a}$, Antonia Micucci ${ }^{a}$, Giorgia Ponsia, c, Fabio Paglieria, ${ }^{2}$, \\ Elsa Addessia \\ aUnità di Primatologia Cognitiva, Istituto di Scienze e Tecnologie della Cognizione, CNR,

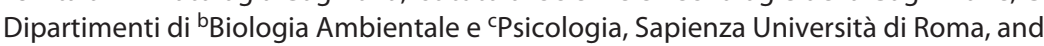 \\ ${ }^{\mathrm{d} G o a l-O r i e n t e d ~ A g e n t s ~ L a b, ~ I s t i t u t o ~ d i ~ S c i e n z e ~ e ~ T e c n o l o g i e ~ d e l l a ~ C o g n i z i o n e, ~ C N R, ~}$ \\ Rome, Italy \\ E-Mail: francesca.depetrillo@uniroma1.it
}

When faced with choices between smaller, sooner options and larger, later options, humans show a magnitude effect, according to which larger rewards are discounted over time at a lower rate than smaller rewards. Most of the studies carried out on non-human animals have led to

6th European Federation for Primatology Meeting
Folia Primatol 2015;86:235-386 DOI: $10.1159 / 000435825$ 
negative results. Here, we tested 10 capuchins in a Delay Choice Task in which we manipulated either the amount or the quality of food rewards. Specifically, we aimed to evaluate whether capuchins showed a magnitude effect when they chose between different quantities of the same food and how food quality affected their temporal preferences. Whereas food quality did not affect capuchins' temporal preferences, we found the first evidence of an effect of the reward quantity on temporal preferences in non-human primates. In contrast with the results obtained in humans, capuchins' choices of the larger later option decreased as the amount of the smaller sooner option increased, showing a 'reverse' magnitude effect, as already observed in rats. This suggests that capuchins based their temporal preferences on the quantity of the smaller sooner option, rather than on that of the larger later option, probably because in the wild waiting may be costly. In fact, during the delay the larger option could be stolen from a competitor, a predator may force the individual to abandon it, and there is a cost associated to all the lost opportunities to invest an option that is not yet available. Moreover, wild individuals virtually never have to choose between a smaller immediate option and a larger later option at the same time, but they more often encounter them consecutively. Hence, since the first information they have is about the amount (or quality) of the smaller option, paying attention and deciding on the basis of its features may be an adaptive strategy rather than an economically irrational response. This study complied with the Directive 2010/63/EU and was approved by the Italian Health Ministry.

\title{
Visual Memory for Compound Stimuli in Tufted Capuchin Monkeys (Sapajus spp.)
}

\author{
Diego Antonio De Simone ${ }^{a, b}$, Carlo De Lilloc, Valentina Truppa ${ }^{a}$ \\ anstitute of Cognitive Sciences and Technologies, National Research Council (CNR),

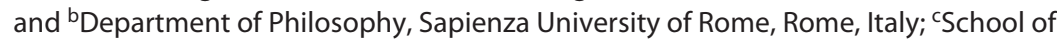 \\ Psychology, University of Leicester, Leicester, UK \\ E-Mail: diegoantonio.desimone@gmail.com
}

Non-human primate species are less proficient than humans at processing global properties of visual compound stimuli. It has been suggested that humans preferentially process stimuli globally because this enables a more economical encoding of the stimuli. In this study, we assessed the role of short-term memory in global/local processing by presenting tufted capuchin monkeys (Sapajus spp.) with Navon-type hierarchical figures in both simultaneous and delayed matchingto-sample tasks. The capuchins' ability to discriminate compound stimuli was evaluated as a function of increasing delay intervals $(0.5,1.0,2.0$ and $3.0 \mathrm{~s})$ between the disappearance of the sample and the presentation of the comparison stimuli. The results showed that recognition of local features was above chance level with delays of up to $3.0 \mathrm{~s}$. By contrast, accuracy for global configurations had a more rapid decay and was above chance level only in simultaneous, 0.0 and $0.5 \mathrm{~s}$ delay conditions but not with delays of $1.0 \mathrm{~s}$ or longer. These findings indicate that capuchins' propensity to process the local properties of visual stimuli extends to short-term memory and cannot be reversed by increasing delays intervals. Significant effects of stimulus size and order of presentation of global/local trials were also found. This research complied with the European Directive 2010/63/EU for the Use of Nonhuman Primates in Research. The research protocol was approved by the Italian Health Ministry (Central Direction for Veterinary Service, approval No. 11/2011-C). 


\title{
Birth in Bonobos (Pan paniscus): Female Cohesiveness and Emotional Sharing
}

\author{
Elisa Demuru, ${ }^{a, b}$, Pier Francesco Ferraric ${ }^{c}$, Elisabetta Palagi ${ }^{b, d}$ \\ aDipartimento di Bioscienze, Università di Parma, Parma, bMuseo di Storia Naturale, \\ Università di Pisa, Pisa, 'Dipartimento di Neuroscienze, Università di Parma, Parma, and \\ dUnità di Primatologia Cognitiva, Istituto di Scienze e Tecnologie della Cognizione, \\ Consiglio Nazionale delle Ricerche, Roma, Italy \\ E-Mail: elidemu@yahoo.it
}

Sociality around birth has been proposed as a unique feature of our species and traces its origin to the very beginning of human evolution. In traditional societies, the social and practical support to the parturient is almost exclusively provided by women, with absolute cultural taboos preventing the participation of men. Humans surely differ from their closest living relatives in the imperative need for assistance during delivery that is linked to some distinctive traits of birth in our species. Yet, considering the social, cognitive and emotional complexity of many primate species, it is plausible that they may show a certain degree of sociality around birth. It is nowadays impossible to draw a reliable picture of the social dynamics around birth in monkeys and apes since the only available reports on the behaviour of group members during deliveries are scattered and anecdotal. Here we describe three daytime births in bonobos (Pan paniscus). The observation conditions of the captive environment allowed us to film and quantitatively describe the behaviours of the whole group. Our data show that birth in bonobos is a peculiar social event in which females are particularly concerned. Indeed, females stay closer to the parturient than males do and the frequency of affiliative interactions is higher within female-female dyads, whereas the frequency of agonistic interactions is higher within female-male dyads. Moreover, the more dominant and elderly females also provided a sort of 'assistance' to the parturient, by performing the same gesture performed by her to grab the infant during its birth, although only the mother grabbed the infant at the delivery. These results show that the bonds linking bonobo females are also reinforced during rare events, such as births. We suggest that the collective support of females in bonobos might be one of the building blocks of more complex and culturally shaped forms of sociality which are expressed during the delivery event in humans.

\section{Playing Hide and Seek with Primates: A Comparative Study of Theory of Mind}

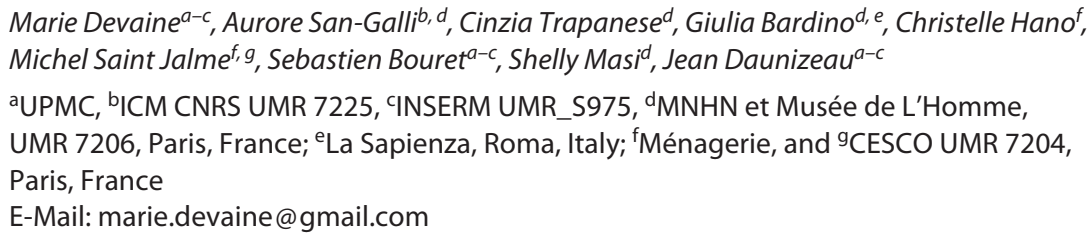

The ability to anticipate another individual's behaviour is likely to be critical for survival. Humans deal with this problem by deciphering others' hidden intentions, which has been coined Theory of Mind (ToM). In the context of mutual social interactions, this induces the update of recursive beliefs: I think that you think that I think... However, there is no consensus on whether nonhuman primates are endowed with such sophisticated ToM. In this work, we ask whether primate species differ in terms of ToM sophistication. The main novelty of our approach is to evaluate primates' ToM sophistication from the sequence of their choices in a game calibrated using information theory. We tested seven species including great apes (gorillas, chimpanzees and orangutans), monkeys (two species of macaques and sooty mangabeys) and prosimians (Le-

6th European Federation for Primatology Meeting
Folia Primatol 2015;86:235-386 DOI: $10.1159 / 000435825$ 
mur catta) housed in different zoos and one lab. Our experimental paradigm is essentially a competitive game against a human opponent. At each trial, the individual has to guess where the human opponent has hidden the food (either in his right or left hand). Critically, the food location is chosen online by algorithms, whose artificial ToM sophistication is experimentally controlled, yielding three different conditions: a control (a simple random biased sequence), a Bayesian learning algorithm (using no ToM) and a meta-Bayesian ToM agent (performing one step of inference of the type 'I think that you think'). Performance results show that most non-human primates win in the control condition but lose against the ToM agent, in contrast to our previous results in humans (who won). Surprisingly, great apes do not perform better than the other nonhuman species. However, computational analyses of choice sequences reveal that while the behaviour of monkeys and prosimians seems driven by simple learning strategies, great apes' behaviour is closer to the one of ToM agents, which would assume a cooperative interaction with the experimenter.

\section{Emotional Propagation in Humans: The Cases of Fear and Joy}

\section{Guillaume Dezecache}

Institut Jean Nicod, Institut d'Etudes Cognitives, Ecole Normale Supérieure, Paris, France E-Mail: guillaume.dezecache@gmail.com

Crowd psychologists of the 19th and 20th centuries have left us with the idea that emotions are so contagious in humans that they can cause large groups of individuals to rapidly and spontaneously converge on an emotional level. Good illustrations of this claim include situations of crowd panic where large movements of escape are thought to emerge through local interactions, and without any centralized coordination. In this talk, I will discuss the propagation of two allegedly contagious emotions (fear and joy) at two different levels of analysis: at a proximate level of analysis (the how-question), I discuss the potential mechanisms underlying the transmission of these emotions, and the extent to which emotional transmission can be considered analogous to a contagion process. To this end, I will present data suggesting that fear and joy can be transitively contagious, i.e., be transmitted from an individual A (expressing fear and joy), to a participant $\mathrm{C}$, who is observing another participant (B) watching the emotional content contained in A. Secondly, at the evolutionary/ultimate level of analysis (the why-question), I ask why humans seem to be so inclined to share their emotional experience of fear and joy with others. The production of spontaneous displays of emotions when confronted with emotional stimuli may, I will hypothesize, have evolved for the purpose of communicating adaptive-value information to others. I will present the preliminary results of an experiment which sought to investigate whether spontaneous facial reactions are sensitive to others' informational demands. Finally, I will discuss the potential mechanisms of emotional transmission in chimpanzees and to what extent they might differ from those found in emotional transmission among humans. The research I will present complied with the European Directive 2010/63/EU and ethics approval was obtained from the local research ethics committees (CPP Ile de France III). 


\title{
Regeneration Patterns of Large-Seeded Plants Support the Key Role of Collared Brown Lemurs (Eulemur collaris) for the Floristic Diversity of the Malagasy Littoral Forest
}

\author{
Giuseppe Donati ${ }^{a}$, Luciano Atzeni ${ }^{b}$, Alejandra Duarte ${ }^{c}$, Jonny Rabenantoandro ${ }^{d}$, \\ Faly Randriatafika ${ }^{d}$, An Bollen $^{e}$ \\ aNocturnal Primate Research Group, Department of Social Sciences, Oxford Brookes \\ University, Oxford, and bepartment of Geography, University College of London,

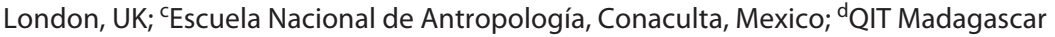

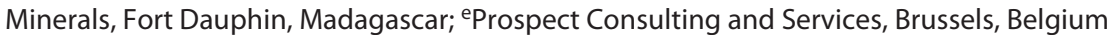 \\ E-Mail: gdonati@brookes.ac.uk
}

Malagasy littoral forests contain $13 \%$ of the island's floristic diversity and approximately $4 \%$ of its endemic species. In the south-east of the island, this habitat has been exposed to heavy fragmentation due to shifting cultivation and logging. This area faces an additional risk due to the mining of titanium deposits. Considering these threats, it is crucial to understand the key drivers of forest restoration and habitat corridors. As the largest seed disperser in this ecosystem, the collared brown lemur (Eulemur collaris) is known to disperse seeds of up to $36.5 \mathrm{~mm}$ in length. We thus hypothesize that the disappearance of this lemur may cause cascade effects for large-seeded plant regeneration. We investigated this hypothesis using three forest fragments of a similar size: one with high densities of E. collaris, one with low densities of E. collaris, and one in which the species disappeared long ago. Regenerating saplings, ranked by classes of height, were sampled in a total of 300 small plots of $2 \times 2 \mathrm{~m}$ each. At each point, mature trees were also recorded via the point-quarter method to control for the presence of parent trees. Differences between fragments in the frequencies of the overall regeneration, lemur dispersed species, and Eulemur dispersed species were explored through a Generalized Linear Model with negative binomial distribution. Lemur and Eulemur dispersed plant species were identified on the basis of observational data and seed size. The results showed that both overall regeneration and the regeneration of the lemur dispersed species did not differ between fragments. In contrast, the two fragments in which E. collaris persists showed higher regeneration of tree species exclusively dispersed by this lemur, as compared to the fragment in which the species has been eradicated. This work demonstrates the key role of medium/large frugivorous lemurs for the maintenance of forest diversity in Madagascar.

\section{Does Mass Distribution Reflect the Manner in Which Primates Move? An Integrative Study Using the Baboon as a Model}

François Druelle ${ }^{a, b}$, Peter Aerts ${ }^{a}$, Gilles Berillon ${ }^{b}$

aLaboratory for Functional Morphology, University of Antwerp, Antwerp, Belgium; bUPR 2147, CNRS Laboratory, Paris, France

E-Mail: francois.druelle@student.uantwerpen.be

This study assesses the functional relationships between morphology and locomotor habits in primates. We focus on infancy which shows dramatic morphological and locomotor changes. This enables testing of the assumption proposed by previous studies, i.e. the changes in locomotion, in terms of time-budget and movement pattern, are correlated with body mass distribution through ontogeny. Indeed, it is commonly seen that the least-energy consuming adaptation in mammals corresponds to a body shape adapted for the preferred locomotor mode. However, regarding the diversity of the movement capacities of non-human primates, the understanding of their morphological specializations is a challenge. Their evolution in a complex environment 
adapted their body to multiple mechanical requirements. Therefore, studying both the biomechanical and behavioural implications of changing body shape during ontogeny may help to understand better how the primates' morphology enables them to move the way they do. We collected accurate quantitative data on locomotor behaviour in conjunction with morphometrics from a sample of 7 infant olive baboons (Papio anubis). These baboons are raised at the Primatology Station of the CNRS (France) where they live inside the same social group. Individual locomotor behaviour was quantified using the focal sampling method. The body mass distribution was estimated according to a geometric model based on external measurements. Multivariate analyses on both segmental masses and locomotor modes (proportion) enabled us to assess their relationships. The results reveal that individuals with more proximal mass in the limbs use more quadrupedal locomotion; individuals with more distally concentrated mass use more locomotion that necessitates grasping abilities. This research is part of an ongoing long term study that aims to test the general hypothesis that body shape might reflect locomotor adaptations. This research compiled with the European Directive 2010/63/EU.

\title{
Investigating Infection Risk and Sociality: Centrality Interacts with Seasonality to Predict Lice Load in Free-Ranging Female Japanese Macaques, Macaca fuscata
}

\author{
Julie Duboscq ${ }^{a, b}$, Valeria Romano ${ }^{a, b}$, Cédric Sueur $^{a-c}$, Andrew MacIntosh $^{d, e}$ \\ ${ }^{a}$ Centre National de la Recherche Scientifique, Département Ecologie, Physiologie

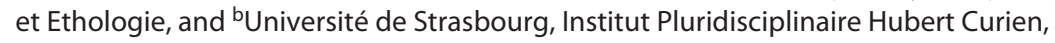 \\ Strasbourg, France; ' Unit of Social Ecology, CP231, Université libre de Bruxelles, Campus \\ Plaine, Brussels, Belgium; ${ }^{d}$ Kyoto University Primate Research Institute, Inuyama, and \\ eKyoto University Wildlife Research Centre, Kyoto, Japan \\ E-Mail: julie.duboscq@iphc.cnrs.fr
}

Lice are socially-transmitted ectoparasites and potential vectors of disease. The infection risk of individuals depends partly upon their degree of contact with others. While grooming may facilitate transmission of ectoparasites via body contact, it may constrain the spread of such organisms through parasite removal. Japanese macaques conspicuously pick out louse eggs during grooming, a behaviour we used to estimate individual lice load non-invasively. To investigate parasite/disease risk in relation to degree of sociality, we tested the relationship between lice load and contact/grooming centrality. We made two opposing predictions: (i) central individuals harbour more lice because of their numerous contacts within the network; (ii) central individuals harbour fewer lice because of their frequency of being groomed. We studied 20 females of one group in Koshima, Japan. To account for variation in centrality and lice load, we controlled for each female's dominance rank, her reproductive state, and for season. Results show that centrality interacts with seasonality to predict a female's lice load: less central females harboured more lice than more central females in the mating (winter) and birth (summer) seasons but the relationship reversed during other seasons. We discuss these results with regards to the biology of parasite and host. We reflect on the relation between infection risk from socially-transmitted parasites/diseases and degree of sociality. Our estimation of lice load constitutes an ideal substitute to invasive methods for natural populations. This research complied with the International Primatological Society (IPS) Guidelines for the Use of Nonhuman Primates in Research. 


\title{
Intrachromosomal Telomeric Sequences Distribution in Ceboidea Can Reveal Insights into the Chromosomal Evolution in New World Primates
}

Francesca Dumas, Luca Sineo

Dipartimento di Scienze e Tecnologie Biologiche, Chimiche e Farmaceutiche, Università

degli Studi Palermo, Palermo, Italy

E-Mail: francesca.dumas@unipa.it

The high intra- and intergeneric genome variability, due to intra- and interchromosomal rearrangements that occurred during Platyrrhini radiation, can possibly be linked to the Interstitial Telomeric Sequences (ITSs). ITSs found between the end of the chromosomes and centromeres, traditionally considered just 'junk DNA', seem, instead, to have a role in chromosomal evolution. For this purpose, the intrachromosomal location of telomeric sequences has been analysed by fluorescent in situ hybridization with the telomeric (TTAGGG)n sequences (synthetic PNA probe) on the following New World primates: Callithrix argentata, Callithrix jacchus, Cebuella pygmaea, Saguinus oedipus, Saimiri sciureus and Aotus nigriceps. All telomeres were mapped with the PNA probe in all species; furthermore our results and data from the literature showed that ITS, centromeric and interstitial, are present in Aotus. We underline that ITSs may play a rule in the karyotypic evolution of the Aotus genus and/or provide phylogenetic information.

\section{Molecular Cytogenetics as a Tool for Primate Taxonomic Identification: A Case Study in Captivity}

\author{
Francesca Dumas ${ }^{a}$, Maricò Maone ${ }^{a}$, Luca Sineo ${ }^{a}$, Takifumi Ishida ${ }^{b}$
}

a'Dipartimento di Scienze e Tecnologie Biologiche, Chimiche e Farmaceutiche (STEBICEF), Università degli Studi di Palermo, Palermo, Italia; ${ }^{b}$ Graduate School of Science, University of Tokyo, Tokyo, Japan

E-Mail: francesca.dumas@unipa.it

Primates in zoos and parks are often kept in groups of animals of different origin, without any information regarding the biological and genetic aspects that characterise them. This often results in incorrect identification of the species and consequently, incorrect management. This paper presents a case of taxonomic identification by molecular cytogenetic analysis of New World primates (Platyrrhini), generically classified as Aotus trivirgatus, from a primate research centre in Tokyo. The classification of Aotus is controversial: indeed it was initially recognized as a single species Aotus trivirgatus; then, based on the colour of the hair on the neck, on the different susceptibility to malaria and on cytogenetic data, up to eleven species have been identified, some of which are morphologically indistinguishable but have different karyotypes and several polymorphisms. The hybridization in sympatric condition complicates the picture. In breeding and conservation programmes, the $\mathrm{G}$ banding reconstruction of karyotypes is the approach of choice in the identification of most of the species of the genus Aotus. We therefore applied cytogenetic methods, such as Cbanding, G-banding and NOR characterization and also Fluorescence in situ Hybridization (FISH) with some human chromosome probes used as markers, on Aotus; metaphases were prepared, following standard cytogenetic protocols. The results allowed us to determine the species and subspecies of the samples analysed as Aotus lemurinus griseimembra $(2 n=54 / 53)$. In addition, the present work allowed us to demonstrate the validity of the cytogenetic approaches as useful tools in taxonomic identification. The chromosomal analysis, together with the morpho-functional studies, represents an alternative diagnostic methods to the genome analysis, applicable both in the maintenance of animal colonies in captivity and in the identification of species in the wild.

6th European Federation for Primatology Meeting
Folia Primatol 2015;86:235-386 DOI: $10.1159 / 000435825$ 


\title{
Evaluating the Impact of Primate Education Workshops on Children's Learning by a Pre- and Post-Test in Tabasco, Mexico
}

\author{
Karen Ahlem Esper-Reyes ${ }^{a}$, Francisca Vidal-García ${ }^{b}$ \\ aUniversidad Autónoma del Estado de Morelos, Cuernavaca, and ${ }^{b}$ Red Biología y \\ Conservación de Vertebrados, Instituto de Ecología A.C., Xalapa, Veracruz, México \\ E-Mail: ahlemesper8@gmail.com
}

The three species of Mexican primates (Alouatta pigra, A. palliata and Ateles geoffroyi) are the most northern primates in the American continent. They are considered to be endangered due to habitat loss, fragmentation and human activities. Tabasco is the region of Mexico in which the three species are present. These species have been studied for their role in forest regeneration, because they are primary seed dispersers of a great variety of plants. It is necessary to establish actions for their conservation. Environmental education can be a tool for studying and understanding relationships and interactions between primates and non-human primates. It is a process by which individuals and local communities become aware of their environment; it is a teaching mechanism that helps people to acquire ethical values, skills and willingness to act on the resolution of current and future environmental problems. The aim of this project was to evaluate the impact of environmental education workshops on children by using pre- and postlearning tests. We designed a knowledge test and a workshop with information about Mexican primates. We carried out the workshop in six groups of 30 children, between 10 and 12 years old, for a total of 180 students. The knowledge test was applied before and after the workshop. Each test was scored, and scores were compared by using a t-student test. Results showed that children improved their knowledge in terms of biology, distribution, and the importance of the conservation of these endangered species after the environmental education workshop.

\section{Underground Honey Extraction by Chimpanzees, Honey Badgers and Forest Elephants in Loango National Park, Gabon}

Vittoria Estienne, Christophe Boesch

Department of Primatology, Max Planck Institute for Evolutionary Anthropology, Leipzig, Germany

E-Mail: vittoria_estienne@eva.mpg.de

The exploitation of the same food resources by multiple species can result in the adoption of different strategies by different competitors. Recent studies highlighted the relevance of competitive interactions occurring among a wide range of taxa inhabiting one area. We focus on a particular case of indirect competition in Loango National Park (Gabon), where central African chimpanzees (Pan troglodytes troglodytes), forest elephants (Loxodonta cyclotis) and honey badgers (Mellivora capensis) compete for a high-quality, hidden resource: the honey and brood of ground dwelling melipone bees (Meliplebeia lendliana). The underground nests pose a unique challenge for predators, since they are difficult to detect and reach. The three mammalian species that consume this resource in Loango can rely on different morphological or behavioural adaptation to out-compete each other. We use footage recorded by remote video-camera traps over 59 months and ecological data to: document the extractive behaviour of underground nests by the three identified consumers; evaluate which factors affect chimpanzees' choice to dig or not; and investigate which environmental factors affect the success of predators. Chimpanzees visit the bee nests more often than the other two species. The frequencies of success are comparable between chimpanzees and honey badgers, whereas elephants are rarely successful. Chimpanzees and honey badgers show a seasonal activity pattern around the bee nests. Chimpanzees' digging behaviour seems to be inhibited by the previous visit of honey badgers. The main constraint to a suc- 
cessful extraction is the distance between the entrance tube and the location of the underground nest. Our results demonstrate the value of applying remote monitoring methods to investigate the behavioural ecology of target species and to describe previously unreported behaviours. This study complied with the International Primatological Society Guidelines for the Use of Nonhuman Primates in Research.

\title{
Influence of Grasping Ability on Forelimb Long Bone Shape in Prosimians
}

\author{
Anne-Claire Fabre ${ }^{a}$, Louise Peckre ${ }^{b}$, Christine Wall ${ }^{a}$, Anthony Herrel ${ }^{b}$, Emmanuelle Pouydebat ${ }^{b}$ \\ a'Department of Evolutionary Anthropology, Duke University, Durham, N.C., USA; \\ bUMR 7179 C.N.R.S/M.N.H.N, Paris, France \\ E-Mail: fabreac@gmail.com
}

The grasping hand is one of the key morphological hallmarks of human evolution. Our understanding of the human grasping hand is largely built on our interpretation of the specialized primate grasping hand (e.g. the presence of nails instead of claws and skeletal evidence for the presence of manipulative capabilities in early primates using the fine branch milieu). Yet, a holistic approach including the whole forelimb and its behavioural context in grasping and manipulation is currently missing. In this study, we link grasping and manipulation ability related to the shape of the forelimb in a broad phylogenetic context in lemurs. We analysed behavioural data for 16 species of prosimians at the Duke Lemur Center. In addition, we performed surface geometric morphometrics analyses on the forelimb long bone shape (quantified from museum skeletal collections). Next, we linked the behavioural data to anatomical data to evaluate the strength of the relationship between grasping behaviour and forelimb bone shape in primates. The data obtained greatly extend our understanding of the evolution of forelimb morphology in primates in relation to grasping and manipulation behaviour. This quantitative and novel approach is promising and will allow us to infer the grasping behaviour of fossil species in a quantitative way, thus opening up exciting new avenues of research into our understanding of primate evolution focusing initially on lemurs. All animal handling was performed according to protocol IACUC \#A089-14-04 (PI: A.-C. Fabre) complying with the International Primatological Society (IPS) Guidelines for the Use of Nonhuman Primates in Research.

\section{Social Influence on Cognitive Performance in Baboons (Papio papio)}

\section{Joël Fagot}

CNRS, Aix-Marseille University, Marseille, France

E-Mail: Joel.fagot@univ-amu.fr

Physical and social cognition are often studied in different experimental contexts, and little is known on how these two aspects of primate cognition interact. Using automated learning devices for monkeys (ALDM, Fagot \& Paleressompoulle, 2009, Behavioural Research Methods, 41, 396-404), baboons are tested in my laboratory on computerized tasks while they are maintained in their social group. This procedure allows a direct examination of how the social context influences performance. During this talk, I shall highlight several sources of social influence on individual performance. I shall show that the mere presence of others can either enhance or worsen a baboon's performance, depending on the subject's familiarity with the task, I will show that positively and negatively valenced social interactions have different effects on response speed and accuracy, and, finally, will demonstrate the existence of social comparison processes, also affecting the performance of the task. 


\title{
Disentangling Different Contexts of Mother-Offspring Conflict in Orangutans
}

\author{
Sonja Falkner, Julia Kunz, Caroline Schuppli, Anna Marzec, Carel van Schaik, \\ Maria van Noordwijk \\ Anthropological Institute and Museum, University of Zurich, Zurich, Switzerland \\ E-Mail: sonja.falkner@uzh.ch
}

Parent-offspring conflict arises when parents and offspring disagree over the amount of parental investment required. So far, most studies have focused on weaning conflict. However, in some species more sources of conflict are known or predicted. Slow life histories and long interbirth intervals make orangutans a particularly suitable study species to disentangle different sources of conflict. These traits have the advantage that each phase of infancy is more prolonged and thus different kinds of conflict which would coincide in species with faster life histories can be teased apart. In this study we examined mother-offspring conflict in Sumatran (P. abelii) and Bornean orangutans (P. pygmaeus wurmbii). The aim was to describe and distinguish different contexts of conflict in relation to different factors such as offspring age, food availability and site. Simultaneous mother and offspring data were collected during nest-to-nest follows, using 2-min interval focal sampling and detailed ad libitum sampling of potential conflict situations. In total, $558 \mathrm{~h}$ of detailed data at the field site Suaq Balimbing, Sumatra and $933 \mathrm{~h}$ of data at Tuanan, Borneo on 17 immatures ranging from 4 to 113 months of age were collected. We could distinguish three main contexts of behavioural conflict: nutrition, locomotion and proximity. Conflict frequencies were predicted best by offspring age. No effect of food availability or site was apparent, except in gap crossing conflicts, which were slightly more common at Suaq Balimbing. Differences in forest structure between the two sites could explain this result. The lack of rejections during final weaning indicates that milk provisioning gradually declines without active behavioural inhibition on the mother's part. In conclusion, we found that there are indeed different contexts of conflict that peak according to the competence of the offspring, but are also driven by ecological conditions, and that classic weaning conflict is reduced.

\section{Flexible Use of Copulation Calls in Parous and Nulliparous Female Chimpanzees}

\author{
Brittany Fallon ${ }^{a, b}$, Christof Neumann $^{b}$, Richard Byrne $^{a}$, Klaus Zuberbühler $^{a, b}$ \\ aschool of Psychology and Neuroscience, University of St Andrews, St Andrews, UK; \\ 'Institute of Biology, University of Neuchâtel, Neuchâtel, Switzerland \\ E-Mail: blfallon@st-andrews.ac.uk
}

Female chimpanzees prefer to mate with dominant individuals, and one way in which they enact this preference is by producing copulation calls to incite male competition. Male chimpanzees, however, preferentially mate with older, parous females - a strategy that is maintained using long-term sexual coercion. Given males' interest in parous females, it is not clear whether lowranking, nulliparous females use the same advertisement strategy as parous females. We hypothesized that nulliparous females employ copulation calls differently from parous females, in order to increase male interest. Additionally, as parous females are typically targeted for coercion, we hypothesized that parous and nulliparous females would call differently in the presence of dominant males. We analysed 1,295 copulation events from 13 parous and 7 nulliparous females in the Sonso community in Budongo Forest, Uganda to investigate which factors influenced female calling behaviour. Data were collected during a period of unusual overlap in female menstrual cycles, which resulted in up to 10 females in oestrus simultaneously. We hypothesized that females, particularly less attractive nulliparous females, would adjust their calling behaviour when 
competing with other oestrus females. Using a generalized linear mixed model, the calling behaviour of parous and nulliparous females was found to significantly differ according to rank of male partner, copulation length and number of dominant males present. There was also a trend for females to increase the likelihood of calling as competition increased. We suggest that this strategy further incites male competition while detracting attention from other females. Overall, we found that nulliparous and parous females employ different calling strategies in order to maximize their chance for reproductive success. All methods received ethical approval from the University of St Andrews and complied with the International Primatological Society Guidelines for the Use of Nonhuman Primates in Research.

\author{
Sexual Bias in Tool Use by Capuchin Monkeys \\ Tiago Falótico ${ }^{a, b}$, Eduardo B. Ottonia \\ anstitute of Psychology, University of São Paulo, São Paulo, Brazil; bRLAHA, School of \\ Archaeology, University of Oxford, Oxford, UK \\ E-Mail: tfalotico@gmail.com
}

Robust capuchin monkeys (Sapajus sp.) are the only Neotropical primates that use tools in the wild. Further, the Serra da Capivara National Park (SCNP) capuchins are the only known population that uses stone tools for more purposes than to crack encapsulated fruits and seeds. They also regularly use sticks as probes. Most stone tools are used by both sexes, but the frequency of use is almost always higher in males, even if both sexes eat the same resources. An exception is stone throwing behaviour during sexual displays. Males were never seen throwing stones. This female bias is easily explained by that fact that only females solicit and stone throwing is used by females to attract male attention. An opposite sex bias is seen in probe tool use. Although in SCNP both sexes consume the same prey and honey, only males are known to use probe tools to obtain these resources. Since a dietary bias is not a likely explanation for this male bias, we examine two other hypotheses: (1) learning opportunity, females may have fewer opportunities to observe tool use behaviour and (2) manipulation propensities, females may be less inclined to manipulate objects in general, and particularly sticks. Initial ontogeny studies of the SCNP population suggest that the difference in female and male manipulation of sticks is present from the first months of life, thus supporting the manipulation hypothesis. This research complied with the International Primatological Society (IPS) Guidelines for the Use of Nonhuman Primates in Research and had the approval of the Brazilian environmental agency (IBAMA - 14825-1).

\title{
The Function of Chimpanzee 'Waa' Barking in Agonistic Contexts
}

Pawel Fedurek, Klaus Zuberbühler

Institute of Biology, University of Neuchâtel, Neuchâtel, Switzerland

E-Mail: pawel.fedurek@unine.ch

In primates, victims of aggression often produce screams and it is believed that the main function of this behaviour is to recruit support from the audience. Data from chimpanzees seem to support this hypothesis since the production of screams is sensitive to fine details of the composition of the audience. Victims of aggression also often produce another type of call, 'waa' barks, and these calls are usually given immediately after screams. However, little is known about the adaptive function of these vocalisations. The aim of this study was to explore the function of

6th European Federation for Primatology Meeting
Folia Primatol 2015;86:235-386

DOI: $10.1159 / 000435825$ 
victims' waa bark calls and to examine whether it differs from that of screams. Data indicate that the production of screams, but not waa barks, was dependent on the composition of the audience. Victims were more likely to physically retaliate against, and less likely to reconcile with, aggressors after waa baking but not screaming. In addition, victims were more likely to produce waa barks, but not screams, when they had already received support from party members. These results suggest that victims' waa barking has a different function than screaming: while the function of screams is to recruit support from bystanders, waa barks are aggressive signals directed at the aggressor, probably to prevent further attack. We conclude that victims' scream and waa bark calls, although often produced during the same agonistic events, are complex social signals directed at different types of receivers and fulfilling different functions. The study complied with the European Directive 2010/63/EU and the International Primatological Society Guidelines for the Use of Nonhuman Primates in Research.

\title{
A Demographic Analysis of Strepsirrhine Primate Research in Captivity
}

\author{
Gloria Fernández Lázaro ${ }^{a}$, Sarah Zehr ${ }^{b}$, Enrique Alonso García ${ }^{a}$ \\ ${ }^{a}$ Animal Welfare Group AWSHEL-IAS, Franklin Institute, Research Institute for North \\ American Studies, University of Alcala, Alcalá de Henares, Madrid, Spain; ${ }^{\mathrm{b}}$ Duke Lemur \\ Center, Duke University, Durham, N.C., USA \\ E-Mail: gloria.fernandez@institutofranklin.net
}

According to the IUCN Red List, the lemurs of Madagascar are the most threatened group of mammals in the world. And yet, some lemurs and other strepsirrhine species have been proposed as animal models for aging research. However, the impacts of such proposals have not been able to be fully assessed because of a lack of information about the type, frequency, level of invasiveness and location of all captive research in which these non-human primates are used. The present study provides an overview of strepsirrhine primate research, exploring their use in captivity as reflected by the scientific articles published between 2010 and 2013. Taxonomy, country, area of research, research class and type of institution served as dependent variables. The 174 qualifying articles showed that research involving these non-human primates is more extensive in the United States than in Europe. Species in the Galagidae and Cheirogaleidae families were used more often in invasive studies of neuroscience and metabolism, including studies of aging, while the most commonly used species in non-invasive studies of behaviour and cognition were true lemurs (family Lemuridae). France was the country which conducted the highest number of invasive research projects, and the United States based Duke Lemur Center the institution with the most non-invasive studies. While the number of invasive studies using strepsirrhines is quite low in comparison to those using other non-human primate species, a thorough cost-benefit analysis of experimental use in strepsirrhines is a necessary step toward understanding the broader impacts of research use in these endangered species. Our findings highlight that the use of primates in aging research could represent concerns, specifically for the Cheirogaleidae and Galagidae taxa. We hope that the present study encourages other scientists to continue in the line of the ethics review process that research on primates, including strepsirrhines, requires. 


\title{
Play Picture Making Music Emotional Enrichment (PME) as Wellness Improvement in Captive Non-Human Primates
}

\author{
M. Ferrero ${ }^{a}$, G. Quaranta ${ }^{b}$, M. Mauthe von Degerfeld ${ }^{b}$, D. Bergero ${ }^{b}$, F. Racioppi ${ }^{b}$, \\ F. Luzic, V. Redaellid \\ ${ }^{a}$ ASL TO3, Collegno, bDSV, Grugliasco, 'VESPA, Milan, and dDISTAL, Bologna, Italy \\ E-Mail: mia@alpimedia.it
}

The Play Picture Making Music Emotional Enrichment (PME) is an experimental enrichment proposed for great apes in captivity, through free painting activity, music (hearing music and playing drums), play and interspecies relationships as mediators. A simplified method (music, play and interspecies interaction) was also proposed in a Macaca sylvanus colony in Bernezzo, Italy. PME is based on the hypothesis that an individual's psychological and social welfare could be improved by having fun playing, free expressing oneself through creativity (that tends to integrate perception, thoughts and feelings), interacting with colours and art materials (multilevel stimulant). The artistic and relational mediators also stimulate self and group interaction (human or non-human primates). The method provides free access to materials, precludes any form of coercion and any reinforcement, except for a positive and respectful interaction human to non-human. PME has been used in Ngamba Island Chimpanzee Sanctuary - CSWCT (Uganda) on 11 subjects (5 $9,4 \sigma^{*}, 2$ babies), in 84 work sessions (43 in 2012 and 41 in 2013) of variable length (20-70 min), in the same work area. To evaluate emotional variations (EV) objectively, facial images (FI) of Macaca sylvanus were acquired by a thermo-camera before and during PME sessions. Surface temperature changes (emotional variation index) were codified as bi-dim matrix and elaborated by a devoted interactive software as imageJ (http://imagej.nih.gov/ij/). The PME participants generally proved to really appreciate the work, expressing interest in materials, involvement and enterprising spirit. They had different approaches to the materials, personal styles of painting and a reduction of anxious or compulsive behaviours during the work sessions. FI showed the EV especially on temperate climate days without any direct interaction with subject. Long registration times are needed to select meaningful images. The research reported complied with the European Directive 2010/63/EU and the International Primatological Society (IPS) Guidelines for the Use of Nonhuman Primates in Research.

\section{An Ethical, Autonomous, Automated Tool for Studying Cognition in Primates Living in Social Groups}

\author{
Jonas Fizet ${ }^{a, c}$, Adam Rimele ${ }^{a}$, Thierry Pebayle ${ }^{b}$, Jean-Christophe Cassel', Christian Kelche ${ }^{c}$, \\ Hélène Meunier ${ }^{a, c}$ \\ ${ }^{a}$ Centre de Primatologie de I'Université de Strasbourg, Niederhausbergen, ${ }^{b}$ Centre \\ d'Investigation Neurocognitive et Neurophysiologique, UMS 3489, Université de \\ Strasbourg, and 'Laboratoire de Neurosciences Cognitives et Adaptatives, UMR 7364, \\ Université de Strasbourg, Strasbourg, France \\ E-Mail: jfizet@unistra.fr
}

Discoveries are highly dependent on technological advances. In neurosciences, behavioural studies are crucial in that they can non-invasively measure the part of cognitive processes which are visible. Research on the evolution of behavioural phenotypes through specific neuropsychological tasks has proven to be an effective method to study these cognitive processes. Further, non-human primates are able to learn tasks used for humans, which make them a key model to examine different paradigms of normal or altered cognitive function. We propose to use such an approach through a new automated apparatus. Our experimental device is designed to measure cognitive performances accurately in monkeys living in social groups. It involves operant condi- 
tioning only, which excludes both deprivation and physical constraints. The subjects have an ad libitum access to the device thanks to an automated identification system. The procedures established are thus particularly in agreement with new ethical standards of animal experimentation $(2010 / 63 / \mathrm{EU})$. Tests, as in humans, are provided via a touch-screen interface. Another original feature of our approach lies in the implementation of a new learning process of different cognitive tasks. The difficulty of each task changes in real time according to individual performance so that the entire experimental device operates autonomously. Here we present the conclusions of a pilot study conducted on a small group of rhesus monkeys (Macaca mulatta), designed to validate (i) the new experimental device, as well as (ii) the ability of subjects to learn and perform several cognitive tasks in parallel. We show the results obtained in several tasks targeting working memory (SOSS), visual short-term memory (DMS) and attention (5-CSRTT). Observations will be discussed in the light of the interest of such a tool for studying underlying processes of cognitive functions as well as the multiple interactions between these functions in non-human primates.

\title{
Determinants of Variation in Orangutans' Cognitive Performance: II. Influences of Rearing Conditions and Human Exposure
}

\author{
S.I.F. Forss' ${ }^{a}$, L.A. Damerius' ${ }^{a}$, Z.K. Kosonen ${ }^{a}$, J.M. Burkart ${ }^{a}$, J. Call ${ }^{d}$, D.B. Haun ${ }^{b}$, K. Liebal', \\ B.M. Galdikas ${ }^{e}$, C.P. van Schaik ${ }^{a}$ \\ aAnthropological Institute and Museum, University of Zurich, Zurich, Switzerland; \\ bepartment of Developmental Psychology, University of Jena, Jena, 'Excellence Cluster \\ 'Languages of Emotion', Freie Universität Berlin, Berlin, and dMax Planck Institute for \\ Evolutionary Anthropology, Leipzig, Germany; 'Department of Archaeology, Simon Fraser \\ University, Burnaby, B.C., Canada \\ E-Mail: sforss@yahoo.com
}

Like humans, non-human primates show great individual variation in their cognitive abilities. The present study aims to explain the variation in cognitive performance on a set of problemsolving tasks using an unusually large sample of orangutans (Pongo abelii and Pongo pygmaeus) brought up under dissimilar conditions $(\mathrm{n}=90)$. We performed an experimental study in compliance with the BIAZA standards for zoo housed non-human primates in nine European zoos, and one in four sanctuaries located in Indonesia, in compliance with standards set by the ethics committee of University of Zurich. Zoo groups of orangutans consist of both mother- and handreared individuals. Orangutans housed in sanctuaries represent a diverse range of backgrounds, including wild-born or sanctuary-born, orphans and non-orphans, and with variable duration in the wild before captivity. This creates a large sample of individuals who have experienced dissimilar conditions during ontogeny as well as variation in duration of human exposure. According to the Cultural Intelligence Hypothesis, developmental conditions crucially influence the intellectual capacity of an individual in a quantitative way by opportunities for learning. In addition, the content and nature of learning opportunities are predicted to strongly alter an individual's cognitive level. We performed a set of experiments assessing responses to novel/ familiar food and objects and reactions toward a human stranger, as well as willingness to accept food from a stranger. Based upon these experiments, we created human orientation indices (HOI) to serve as a measurement of variation in reactions among differently raised individuals. These HOI combined with background knowledge of the individuals as well as time of human exposure were then related to the variation in test performance. Our goal was to understand to what extent human orientation and ontogenic conditions explain individual variation in the measured cognitive performance. 


\section{Social Assessment for a Refaunation Project: A Study Case of the Potential Introduction of Howler Monkeys (Alouatta palliata)}

Montse Franquesa-Soler, John Aristizabal, Luis García-Feria, Juan Carlos Serio-Silva

Instituto de Ecología, A.C, Xalapa, Veracruz, México

E-Mail: montse.franquesa@posgrado.inecol.edu.mx

In Mexico, the mantled howler monkey (Alouatta palliata mexicana) is critically endangered; hence, it has become necessary to consider strategies such as refaunation to support their permanence in their natural habitat. While there are many biotic and abiotic factors to consider for a successful introduction, one aspect that has received less attention is the human social assessment. Introducing a population of animals has an impact on the human community and, at the same time, is impacted by the community's interests. Therefore, the refaunation plan should take into account the socio-economic circumstances which may affect the community. Likewise, performing a social evaluation may contribute to the effective design of an environmental education programme for ecosystem restoration plans. The aim of this work was to conduct a community assessment before the introduction of howler monkeys. The study took place in the Biological Reserve of La Mancha, Veracruz (Mexico). 48 open interviews were conducted at the Community of La Mancha (CLM). The structure of the interview had four sections: knowledge and uses of natural resources around them, knowledge and perception of monkeys, knowledge of previous presence of monkeys in the area and perception towards the potential introduction of howler monkeys in La Mancha. Categories were created based on the responses and analysed using descriptive statistics. The CLM perception of primates was influenced by mass media; they did not know the endemic fauna or its ecological function. CLM would like to see monkeys in La Mancha but they think that people probably will harm them. CLM are conscious of the hunting activity in the community and also they think that children would injure the animals to 'have fun'. For that reason, CLM concludes that there is lack of conservation programmes. A participatory intervention with stakeholders is needed, in order to evaluate the benefits and costs to CLM and its natural heritage.

\section{Effect of Environmental Enrichment on a Captive Colony of Chimpanzees (Pan troglodytes verus): Social Behaviours and Learning}

D. Frasson ${ }^{a}, b$, C. Spiezio ${ }^{a}$

aResearch Department, Parco Natura Viva - Garda Zoologica Park, Bussolengo, Italy;

bTacugama Chimpanzee Sanctuary, Freetown, Sierra Leone

E-Mail: dalila.frasson@libero.it

Structural enrichments, a water pool and an artificial termite mound, were introduced in a chimpanzees' enclosure to improve social behaviours and to stimulate termite fishing, a speciesspecific behaviour. The study was carried out on a captive colony of six chimpanzees (Pan troglodytes verus) at Tacugama Chimpanzee Sanctuary, Sierra Leone. In order to highlight the behavioural differences before the installation of the enrichment and afterwards, individual and social behaviours of the members of the colony were observed. The focal animal continuous sampling method was used to collect behavioural data during 15-min sessions for a total of 45 sessions per subject (270 sessions in total) in the period November 2014 - February 2015. Results of this study show that all individuals performed species-specific individual and social behaviours. All chimpanzees seemed to benefit from the enrichment by increasing activity, decreasing abnormal behaviours and showing species-typical tool-using behaviour. In particular, an increase in social play, grooming and termite fishing was observed, mainly in the young subjects of the colony. The age-dependent patterns seem to indicate that young individuals are more willing to learn new 
behaviours and take part in social interactions. In conclusion, structural enrichment seems to be a good tool to improve the subjects' behavioural repertoire and to decrease boredom and inactivity in captive chimpanzees.

\title{
The Development of Social and Spatial Independence: Do Bonobos (Pan paniscus) and Chimpanzees (Pan troglodytes) Differ?
}

Marlen Fröhlich ${ }^{a}$, Paul Kuchenbuch ${ }^{a}$, Gottfried Hohmann ${ }^{b}$, Takeshi Furuichic, Roman M. Wittig ${ }^{b}$, Simone Pika ${ }^{a}$

${ }^{\mathrm{a}}$ Max Planck Institute for Ornithology, Seewiesen, and ${ }^{\mathrm{b}}$ Max Planck Institute for Evolutionary Anthropology, Leipzig, Germany; 'Kyoto University, Kyoto, Japan

E-Mail: mfroehlich@orn.mpg.de

To model the behaviour of early hominoids, a bulk of research has been focusing on humans' closest relatives, bonobos (Pan paniscus) and chimpanzees (Pan troglodytes). Interspecific comparisons proposed a distinct bonobo-chimpanzee dichotomy in social behaviour and development, e.g. concerning aspects such as social bonds, sexuality and agonistic interactions. However, recent reports of considerable inter-site variability of chimpanzee behaviour challenge this dichotomy. In addition, systematic quantitative comparisons of chimpanzee and bonobo behaviour in groups living in natural environments are still lacking. The aim of the present study was therefore to shed further light on this intriguing issue by studying the ontogeny of social and spatial independence in two bonobo (LuiKotale and Wamba, DRC) and two chimpanzee communities (Taï South group, Côte d'Ivoire; Kanyawara, Uganda). We had two questions: First, do social interactions emerge at comparable time points in bonobos and chimpanzees? Second, does spatial independence develop similarly in bonobos and chimpanzees? To answer these questions, we investigated interaction rates and play behaviour as well as mother-infant body contact and proximity. The behaviour of twelve chimpanzee and twelve bonobo infants was recorded during $1,800 \mathrm{~h}$ of observation using a focal/time sampling protocol, which resulted in a total of 8,562 sample points. In terms of social development, our results showed that inter-species differences significantly exceeded inter-site differences. While bonobo infants surpassed chimpanzees in frequencies of social play and interaction in late infancy, chimpanzee infants generally engaged more in object play. In contrast, the ontogeny of mother-infant proximity was strongly influenced by community and mother's experience (i.e. parity). Overall, our findings provide substantial evidence that bonobos and chimpanzees differ in ontogenetic patterns related to social, but not to spatial independence. This purely observational study complied with the IPS Guidelines for the Use of Nonhuman Primates in Research.

\section{Lemur Tunes and Researchers' Boon! Call and Song Classification in the Malagasy Primates}

\author{
Marco Gamba, Olivier Friard, Giovanna Bonadonna, Roberta Righini, Bakri Nadhurou, \\ Isidoro Riondato, Valeria Torti, Cristina Giacoma \\ Università di Torino, Department of Life Sciences and Systems Biology, Turin, Italy \\ E-Mail: marco.gamba@unito.it
}

Vocal repertoire size provides information about the communication systems of a species and its complexity. It is considered to be an indicator of cognitive ability, social complexity and fitness. We applied different methods to the classification of lemur calls. We focused our study 
on the integration and comparison of tools from the field of speech processing with traditional acoustic analysis. The first application we present is the study of the complexity of the vocal repertoire of E. coronatus, E. flavifrons, E. fulvus, E. macaco, E. mongoz, E. rubriventer and E. rufus. The acoustic features were extracted using an implementation of Dynamic Time Warping (DTW) for the pairwise dissimilarity calculation, and the output inspected using cluster analysis. Despite a relatively similar pattern of vocalizations, the overall range obtained by the unsupervised analysis ranged from 9 to 14 clusters. We found that variation in vocal types such as screams, territorial calls and alarm calls may mask variation at a lower level. We then investigated whether we could compare these results with those emerging from a traditional acoustic analysis and multivariate statistical models. The models showed that DTW appears to recognize the formant variation over a particular frequency range, and that it is primarily reactive to call duration. The second application of DTW included phrases emitted by Indri indri during their song. Results demonstrated that DTW is also well suited for the study of lemur vocal sequences, although normalizing duration may impact how calls are then grouped in the cluster analyses. Finally, we consider the shortcomings of current methodologies and prospects for future improvements, including the Mel Filter Cepstral Coefficients design for animal calls. This research complied with the International Primatological Society (IPS) Guidelines for the Use of Nonhuman Primates in Research.

\title{
Grey or Sparkling? Lunatic or Earthly? A Way to Unravel the Personality Dimensions in Cercocebus atys lunulatus
}

\author{
Iván García-Nisa ${ }^{a, b}$, Barbara Sansone ${ }^{a, b}$, Anna Escuinc ${ }^{c}$, Miquel Llorente ${ }^{b, d}$, Sònia Sánchez ${ }^{e, f}$

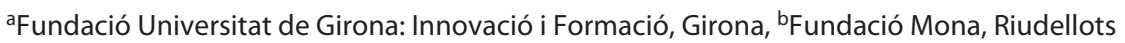 \\ de la Selva, 'Universitat de Barcelona, Barcelona, IInstitut Català de Paleoecologia Humana \\ i Evolució Social (IPHES), Tarragona, and eUniversitat Oberta de Catalunya (UOC), Barcelona, \\ Spain; ${ }^{f}$ Centro de Investigaciones Tropicales de la Universidad Veracruzana, Xalapa, México \\ E-Mail: ivangarcianisa@gmail.com
}

Studies on personality in non-human primates have been traditionally addressed in great apes, our closest relatives. However, a lot of misconceptions and controversies have to be overcome when selecting a method to unveil personality dimensions without falling into anthropomorphism. In addition, when studying personality, the issue of sample size is much debated. Following IPS Ethical Guidelines, our study provides a first approach to the study of personality structure in the white-naped mangabey (Cercocebus atys lunulatus). The five subjects were housed in a group at Barcelona Zoo. Our study combined rating and coding procedures, tested the applicability of conventional measures and made further recommendations for small samples. We based our surveys on two different psychological models (Eysenck Personality Questionnaire 'EPQ' and the Five Factor Model 'FFM') and submitted our data to two reduction methods for a multi-trait/multi-method assessment. We used focal and instantaneous scan sampling to collect behavioural data which served to test the predictive validity of specific and categorical behaviours. We found three potential dimensions for the EPQ-based survey (Extraversion, Dominance, Neuroticism) and five for the FFM (Extraversion, Dominance, Openness, Conscientiousness, Agreeableness). Our results showed that recovering acceptable levels for conventional measures undermines overdetermination in small samples, thus only Extraversion and Dominance dimensions performed appropriately for all tests. All factors were similar to those found in humans and other primates, and statements of construct validity were satisfactorily achieved. We also found more than $70 \%$ of coherent correlations between specific behaviours and personality dimensions suggesting that some behaviours can predict overall personalities and vice versa. However, we suggest that further analyses with a larger sample of subjects would be advisable, and further research is needed to define recommendations for factorial analyses with small samples.

6th European Federation for Primatology Meeting
Folia Primatol 2015;86:235-386 DOI: $10.1159 / 000435825$ 


\section{Challenging Bateman's Dogma: Obligatory Polyandry in Mouse Lemurs}

Fabien Génin, Hajarimanitra Rambeloarivony, Judith Masters

African Primate Initiative for Ecology and Speciation (APIES), Department of Zoology and

Entomology, University of Fort Hare, Alice, South Africa

E-Mail: Fgenin@ufh.ac.za

There is no unified theory of the evolution of mating systems, and the system of serial polyandry observed in small nocturnal mouse lemurs (Microcebus spp.) is particularly difficult to explain in terms of classical sexual selection. Indeed, many hypotheses explaining why females may mate with many males can be rejected (relaxed sexual selection, infanticide and paternal care). We examine the dominant hypothesis of cryptic choice and suggest that the more parsimonious and neutral alternative of induced ovulation - which directly breaks Bateman's dogma as polyandry increases female reproductive success - should be taken into account. In mouse lemurs, males cooperate for encountering elusive females (receptive for only one night) and the fathers of most infants are those that mated with the females about $50 \mathrm{~min}$ after the first copulation. A phylogenetic reconstruction and the presence of a stereotyped courtship indicate that obligatory polyandry in mouse lemurs evolved from a system of dispersed monogamy as the result of progenetic dwarfing, leading to male paedomorphosis (testes regression and loss of territoriality) and female polyestry and multiple ovulation, associated with a shift from a short day breeding system to an unusual long day breeding system. This evolution can be interpreted as a response to environmental hypervariability.

\section{Sexual Dimorphism and Ecological Correlates in Macaca maura (H.R. Schinz, 1825)}

Lavinia Germania, Ngakan Putu Okab, Alessandro Albania ${ }^{a}$ Monica Carosia

aRoma Tre University, Rome, Italy; ${ }^{b}$ Universitas Hasanuddin, Makassar, Indonesia

E-Mail: lav.germani@stud.uniroma3.it

A variety of causal models beyond sexual selection have been suggested in order to explain degree of body mass dimorphism, among which are body size, niche divergence, energetic requirements (e.g., reproduction) and energetic constraints associated with diet. Whereas in monomorphic species costs of male and female body maintenance are equal, in species with positive body mass dimorphism ( $\mathrm{pBMD}$ ), male costs can outweigh those of females. However, this may not hold true if female reproductive costs, in combination with different degrees of $\mathrm{BMD}$, are considered. In fact, when pBMD is slight, female overall costs usually exceed male costs of body maintenance; when pBMD is moderate, female costs may still outweigh, or else counterbalance, male costs. However when pBMD is strong, male costs generally outweigh overall female costs. In macaque species pBMD is reported as being 'moderate to strong' and $\mathrm{Ma}$ caca maura is no exception. Energetic investments and requirements in BMD species may be investigated by studying sex differences in daily activity budgets and diet composition. Behavioural data were collected on a M. maura (IUCN red list; EN) free ranging social group $(\mathrm{n}=31)$ living in Karaenta (Bantimurung Bulusaraung National Park, South Sulawesi) from September to December 2014 (450 group scans). Sexes differed significantly in daily activities ( $p=0.0005)$ with females spending more time than males foraging and feeding $(\mathrm{p}=0.02)$ and less time resting $(p=0.00007)$. While sex did not show an overall effect on diet composition (plants/arthropods/fungi/other), females differed in the higher proportion of arthropods eaten compared to males $(\mathrm{p}=0.0001)$. Preliminary results suggest potential effects of sex on activity budgets and diet composition. 


\title{
Integrated Chimpanzee (Pan troglodytes) and Ecosystem Health in the Greater Gombe Ecosystem, Tanzania
}

\author{
Thomas R. Gillespiea , Dominic A. Travis ${ }^{b}$, Elizabeth V. Lonsdorf \\ ${ }^{a}$ Emory University and Rollins School of Public Health, Atlanta, Ga., b University of \\ Minnesota, Minneapolis, Minn., and ${ }^{\mathrm{C} F r a n k l i n}$ and Marshall College, Lancaster, Pa., USA \\ E-Mail: thomas.gillespie@emory.edu
}

There is widespread concern that infectious diseases pose one of the greatest risks to the survival of apes in the wild. With over fifty years of demographic and behavioural data, thirteen years of SIV prevalence data, ten years of syndromic health (i.e. signs of gastro-intestinal and respiratory illness) and faecal macro- and micro-parasitic data; the chimpanzees of Gombe Stream National Park, Tanzania, are arguably the best characterized wild ape population in relation to health and disease-related threats. A disease risk analysis determined that infectious diseases play an important role in the observed and ongoing decline of the Gombe chimpanzee population, sparking the initiation of a prospective health monitoring system. After more than 12 years of non-invasive screening of chimpanzees for SIVcpz, it is now clear that there are differences in survival and fecundity between SIVcpz+ and SIVcpz- negative cohorts. In addition, mixedmethods approaches have allowed the Gombe Ecohealth team to examine the natural history of this disease and risk factors including opportunistic zoonotic and natural infections that contribute to these differences. This talk will review the successes and challenges of this effort and provide insights as to how findings from Gombe may be applied to other wild primate research and conservation efforts.

\section{The Effects of Early Social Isolation on the Behaviour of a Sub-Adult White-Handed Gibbon Male (Hylobates lar)}

\section{Andrea Giorgi, Giulia Montebovi, Augusto Vitale, Enrico Alleva}

Section of Behavioural Neuroscience, Department of Cell Biology and Neuroscience, Istituto Superiore di Sanità, Rome, Italy

E-Mail: andrea.92.giorgi@gmail.com

In the primatological literature it is reported that early social isolation can result in future permanent behavioural incompetence. The aim of this study was to analyse the effects of early social isolation on the social behaviour repertoire of one white-handed gibbon (Hylobates lar). The species is well known for its sophisticated vocal repertoire and the use of species-specific vocalizations during social encounters. The subject of the present study was a 6 year old male, removed from its normal social environment when it was 2 months old. In order to measure the social competence of the experimental subject, we presented a series of recordings of conspecific vocalizations, obtained from dedicated website, to the gibbon and then introduced a 23 year old conspecific female, initially in her own cage beside his and then into his cage. If social deprivation had had an effect on the male, we would have expected a lack of interest in the vocal playbacks and an aversive reaction towards the conspecific female (i.e., aggressiveness). Our subject did not show any interest in the playbacks coming from two adult males and an adult female, while the reaction towards the introduction of the conspecific female was more positive. After two days of presentation, the two individuals started to spend time in proximity and initiated grooming through the wire-mesh of their respective cages. After four days vocal duets were recorded. The data will be discussed in relation to the possibility of restoring a competent behavioural repertoire in an individual confiscated under C.I.T.E.S., whose personnel closely followed the development of the present study. 


\section{Functional Morphology of Metapodials and Phalanges of Plesiadapis tricuspidens from the Paleogene of France}

Marc Godinot ${ }^{a}$, Séverine Toussaint ${ }^{b, c}$, Dionisios Youlatos ${ }^{d}$

aEcole Pratique des Hautes Etudes, and bUMR 7207 CR2P - C.N.R.S., M.N.H.N., U.P.M.C.-

Paris 6, Département Histoire de la Terre, Muséum National d'Histoire Naturelle, and

CUFR Sciences du Vivant, Université Paris Diderot - Paris 7, Sorbonne Paris Cité, Paris, France; ${ }^{\mathrm{d} A r i s t o t l e}$ University of Thessaloniki, School of Biology, Department of Zoology, Thessaloniki, Greece

E-Mail: godinot@mnhn.fr

Among euarchontan mammals (Euprimates, Scandentia, Dermoptera), Plesiadapiformes constitute an important Paleocene - Eocene group. They play a pivotal role in the debate concerning primate origins. The reconstruction of the locomotor and postural diversity of plesiadapiforms is thus essential for establishing robust phylogenetic relationships within euarchontans. To this end, autopodial elements, being in direct contact with the substrate, are good indicators of positional behaviours and substrate preferences. In this study, we investigated the functional morphology of fossil metacarpals, metatarsals, proximal and intermediate phalanges of Plesiadapis tricuspidens from late Paleocene localities of the Paris Basin, France. These fossil elements were compared to those of extant mammals, and especially squirrels, which exhibit a remarkable positional diversity. We find that $P$. tricuspidens exhibits a mosaic of characters mainly comparable to those of medium-sized arboreal squirrels, confirming previous reports of its arboreality. The functional interpretation of its metapodials provide evidence of a middle foot positioned in parallel to relatively wide arboreal supports as during quadrupedal walking and bounding activities. The metapodio-phalangeal joints reflect movements similar to those observed during grasp climbing on small arboreal supports. The morphology of proximal and intermediate phalanges provides evidence for firm embedding of the terminal phalanges into tree bark, as during claw climbing on vertical supports. It is thus very likely that this species commonly utilized quadrupedal activities and claw climbing and clinging on large horizontal and vertical substrates respectively, as well as some grasping on smaller substrates. In conclusion, as Plesiadapis appears to be among the least specialized plesiadapiforms, it is very likely that arboreal quadrupedal walk, clamber and claw climb and cling may represent the ancestral archontan positional behaviour.

\section{Mammals with Less Seasonal Diets Can Afford Bigger Brains}

Sereina M. Graber, Carel P. van Schaik, Karin Isler

Anthropological Institute and Museum, University of Zurich, Zurich, Switzerland E-Mail: sereina.graber@uzh.ch

The evolution of brain size variation across species is of great current interest. Although there are obvious benefits to having a large brain in terms of enhanced cognitive abilities, brains are energetically extremely costly and thus very sensitive to periods of starvation. Species living in seasonal habitats experience frequent periods of food scarcity, which may affect brain size in two different ways. From the cost perspective, unavoidable starvation may constitute an energetic constraint which prevents species from having large brains ('expensive brain framework'). The benefit perspective, on the other hand, predicts a positive link between brain size and seasonally variable food availability, claiming behavioural flexibility to be the driving force in the evolution of intelligence ('cognitive buffer hypothesis'). Earlier comparative studies in primates have shown evidence that small-brained species experience more seasonal fluctuations in energy intake compared to their large-brained relatives, but on the other hand, large-brained species are better in cognitively buffering their environment. These findings suggest that the two hypotheses, 
the expensive brain framework and the cognitive buffer hypothesis, simultaneously apply to primates. The aim of the current study is to extend these hypotheses to a broader comparative sample of non-primate mammalian species. Data on seasonal diet composition and brain size in 60 species show evidence for a strong evolutionary constraint where mammals with a higher seasonal fluctuation in diet have smaller brains. We further explore the degree of cognitive buffer by taking into account both the environmental and experienced seasonality, and linking it to brain size. With this study we are able to show that ecology plays a major role in the evolution of intelligence, and, in a broader sense, we emphasize the importance of taking into account the cost and benefit perspective when explaining brain size variation across species.

\title{
The Honey-Trap Experiment: Integrating 7 Years of Research from the Sonso Chimpanzee Community of Budongo Forest, Uganda with Theoretical Frameworks of Culture and Tool Use
}

Thibaud Gruber

University of Neuchâtel, Neuchâtel, Switzerland

E-Mail: thibaud.gruber@gmail.com

The question of animal culture has been predominant in the social learning literature, particularly during the last two decades, with chimpanzee data from both wild and captive groups making a substantial contribution to the debate. One way to address this question is to implement field experiments which combine the controlled settings of the laboratory with an ecologically valid context: the natural habitat of wild primates. Today, the debate is no longer on whether chimpanzees have culture or not. Rather, empirical researchers and theorists now attempt to decipher how much chimpanzee cultures compare to the human phenomenon, and the evolutionary relatedness between the two phenomena. Several hypotheses have been developed over the last decades, including the Zone of Latent Solutions (Tennie et al., 2009), or the Necessity and Opportunity Hypotheses (reviewed in Koops et al., 2014), to explain the appearance of tool use. Here, I will use the results of our own research on tool use in the Sonso community of Budongo Forest, Uganda (Pan troglodytes schweinfurthii) to assess these hypotheses. Critically, the Sonso chimpanzees, a community with a leaf-based culture, have proven surprisingly reluctant to learn stick use, a behaviour long classified as universal in chimpanzees. More recent results on the development of a novel tool use behaviour, moss-sponging, suggest that chimpanzees expand their cultural repertoire in the vicinity of what they know already rather than with brand new behaviours. I will also connect this research with other hominines, including bonobos and early human ancestors, to evaluate what features may have changed in our cognitive evolution to make our cultures so much more developed than those of our closest relatives. This research complies with the European Directive 2010/63/EU and the International Primatological Society (IPS) Guidelines for the Use of Nonhuman Primates in Research.

\section{Food Sharing Patterns in Common Marmosets: Who Gets the Food?}

\author{
Eloísa M. Guerreiro Martins, Christa Finkenwirth, Judith M. Burkart
}

Anthropological Institute and Museum, University of Zurich, Zurich, Switzerland

E-Mail: eloisamanuel.martins@uzh.ch

Humans across cultures extensively share food. Among hunter gatherers, mothers and dependent children consume a larger quantity of energy than they are capable of producing. Thus family members adjust their sharing behaviour by providing extra energy to those in need. 
Among primates, extensive food sharing is also present in callitrichid monkeys, who, like humans, engage in high levels of allomaternal care. The aim of this study was to characterize food sharing patterns in the common marmoset (Callithrix jacchus), a callitrichid monkey, to assess similarities and differences to the human pattern. We tested adult members from 6 family groups during 21 months. Testing periods ranged from birth until adulthood of immatures. Every adult was tested twice a week with 3 food sharing trials, resulting in a total of 5,681 trials. The food item in all trials was a cricket. We recorded the behaviour of the food owner, and all food related behaviours by other group members. We analysed (i) who shared with whom, (ii) how they shared (proactive, reactive, tolerated taking), and (iii) whether adults shared more if they had witnessed an immature having difficulty in obtaining a food item. Our results show that immatures received the first food items from adults around 5 weeks of age. They received more than $50 \%$ of all food items between week 8 and 19 after birth. The majority of all shared food items (91\%) were given to immatures. Food sharing between adults was mostly directed at breeding females. The latter shared fewer food items with offspring than other adult group members, which is presumably due to the high energetic costs of simultaneous lactation and gestation. Lastly, if food items were hard to obtain for immatures, adults shared more food, and for a longer period of time. In sum, common marmosets seem to adjust their food sharing behaviour to the need of the receiver by taking age, social status and skill into account.

\section{Visual Illusions in Great Apes, Monkeys and Humans}

Daniel Hanus ${ }^{a}$, Valentina Truppa ${ }^{b}$, Josep Calla

${ }^{a}$ Max Planck Institute for Evolutionary Anthropology, Leipzig, Germany; ${ }^{b}$ Istituto di Scienze e Tecnologie della Cognizione, CNR, Rome, Italy

E-Mail: hanus@eva.mpg.de

It has been argued that humans' susceptibility to visual illusions does not simply reflect cognitive flaws but rather specific functional adaptations of our perceptual system. In order to investigate comparative and developmental aspects of such perceptual phenomena we presented a battery of five different visual illusions to chimpanzee, monkey (capuchins) and human subjects (3-5 year old children and adults). Previous illusion research on non-human subjects mainly focused on a matching-to-sample-paradigm in computerized settings. In contrast, we used a new and more naturalistic approach, where two identical food items were presented on separate trays with the crucial difference being only the appearance of the surrounding area. Subjects always received the content of the tray they selected and were therefore never differentially reinforced. Our results validated the new method as the expected illusionary effects where clearly induced in human adults - at least in four of the five illusions. Whereas the illusionary effect was generally less pronounced in younger children compared to adults, chimpanzees seemed basically immune to it. Capuchin monkeys on the other hand revealed mixed results depending on the particular illusion tested. Even though our results did not generate a uniform and straightforward picture, the data provide empirical descriptions of perceptual phenomena that might help to understand both human and non-human cognition better. 


\section{Treading the Energetic Tightrope: Long-Term Energetics of Bornean Orangutans in Non-Masting Swamp Forests}

Mark E. Harrison ${ }^{a, b}$, Helen C. Morrogh-Bernard ${ }^{b}$, Simon J. Husson ${ }^{b}$, Twentinolosa Firtsman ${ }^{b, c}$, Santiano $^{b, c}$, Wartika R. Farida ${ }^{d}$, Maria A. van Noordwijke, Erin R. Vogelf

a University of Leicester, Leicester, UK; ${ }^{\mathrm{b}}$ The Orangutan Tropical Peatland Project, and

${ }^{c} C e n t r e$ for the International Cooperation in Sustainable Management of Tropical

Peatlands, University of Palangka Raya, Palangka Raya, and ${ }^{\mathrm{d} R e s e a r c h ~ C e n t r e ~ f o r ~ B i o l o g y, ~}$ Indonesian Institute of Sciences, Cibinong-Bogor, Indonesia; eUniversity of Zurich, Zurich, Switzerland; ' Rutgers University, New Brunswick, N.J., USA

E-Mail: mharrison@outrop.com

Food availability, plus diet quality and intake, are considered to be key factors influencing individual fitness and population density in primates. Evaluating the impacts of these on primate populations requires comparisons of populations over space and/or time, using comparable field data collection methods and over a time frame relevant to the species' life history. Obtaining such data sets can be very difficult for long-lived primate species with slow life histories. We collected data on food abundance using tree plots ( $\mathrm{n}=$ mean 1,344 stems), food quality through nutritional analysis of foods eaten ( $\mathrm{n}=183$ items), plus dietary energy intake and behaviour profiles using standardised focal-animal follow methods from 2003-2014 (n >9,000 h) for the wild orangutan (Pongo pygmaeus wurmbii) population in the non-masting Sabangau peat-swamp forest, southern Borneo. This period exceeds the orangutan inter-birth interval of 6-9 years and approaches the age of first production in females ( $~ 15$ years), and is thus relevant in the context of orangutan life history. Analyses reveal variations and inter-relations among these variables over this long time period, providing insight into the influence of these bottom-up processes on individual energetic status and behaviour. Comparisons with a similar dataset collected from 2003-2010 from the wild orangutan population in the nearby, and ecologically similar yet more productive, Tuanan peat-swamp forest yield further insight into the potential influences of these bottom-up processes on orangutan populations, with lower population densities and longer inter-birth intervals reported in the less productive Sabangau site. These analyses enhance our understanding of the influence of environmental food quality and abundance on primate diet quality and intake, and how this in turn may have wider implications at both the individual and population level.

\section{Committed to Conservation: A Biocultural Approach to Chimpanzee Conservation in Uganda}

Jessica Hartel ${ }^{a}$, Brody Sandel $^{a}$, Elizabeth Ross ${ }^{b}$, Jens-Christian Svenning ${ }^{a}$, Casper Andersen $^{a}$, Richard Wrangham ${ }^{c}$, Peter C. Kjærgaard ${ }^{a}$

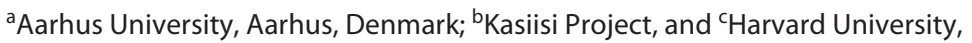

Cambridge, Mass., USA

E-Mail: hartel@cas.au.dk

Challenges of biocultural heritage management are becoming increasingly complex as the growing human population puts heavier demands on land and food supplies. This is particularly clear at Kibale National Park (KNP) in Uganda where an estimated 240,000 people press against the park boundary. The park is rich in biodiversity and is home to one of the largest populations of eastern chimpanzees (Pan troglodytes schweinfurthii). The growth of the human population and competition over land in this area has put increased pressure on KNP to provide resources, forcing some species to seek food and shelter in peripheral villages and crop fields. One of the longest continuous field research projects, the Kibale Chimpanzee Project (KCP), has not only contributed much to our knowledge of chimpanzee ecology, physiology and social behaviour, but

6th European Federation for Primatology Meeting
Folia Primatol 2015;86:235-386 DOI: $10.1159 / 000435825$ 
has also forged strong relationships with the local community by implementing many successful long-term conservation initiatives, including the Kibale Snare Removal Program (KSRP) and the Kasiisi Project (KP) both in 1997. A new research initiative at the Centre for Biocultural History at Aarhus University uses KCP as a test case to analyse how long-term research projects can help promote social, cultural and biological sustainability in Africa. The project combines qualitative interviews, sustainability theory, macroecological GIS analysis and quantitative human-disturbance data to determine how long-term research sites can promote conservation and cultivate sustainable development locally. Satellite data show forest regrowth in the research area from 2000 to 2010, while other areas indicated forest decline. Since KSRP's inception more than 6,500 snares have been removed in KNP, drastically reducing snare presence in the research area and the frequency of chimpanzee snare injuries. Following KP community-based conservation education activities, students showed significant positive attitudinal shifts towards chimpanzees and the forest. Research complied with IPS Guidelines.

\section{Keepers of the Forest: Long-Term Great Ape Research Sites in Uganda Provide Additional Habitat Protection}

Jessica Hartel, Brody Sandel, Jens-Christian Svenning, Peter Kjærgaard

Aarhus University, Aarhus, Denmark

E-Mail: hartel@cas.au.dk

Deforestation is one of the leading threats to great ape populations across equatorial Africa and SE Asia. Orangutans are losing forest to palm oil plantations at unprecedented rates and timber extraction for charcoal, as well as clear cutting for agriculture and livestock, is pushing the iconic Virunga gorillas to extinction. Exponential population growth in Uganda is threatening strongholds of chimpanzee habitat as farmers encroach on protected park and reserve boundaries. Only small fragments of forest remain outside protected areas. Uganda's chimpanzees are eking out an existence in all possible remnants of forest. While chimpanzees can survive in a variety of habitats, primary forests yield the largest, most densely concentrated populations. Conservation of primary forest is therefore critical to wild great ape survival. Long-term research sites are uniquely positioned to invest in sustained preservation of species and habitat, further extending the safeguarding benefits that protected areas and conservation initiatives afford. The Kibale Chimpanzee Project (KCP) was established in 1987 and has been a stable source of research, conservation and educational outreach in Kibale National Park, Uganda. Using MODIS data, tree cover has shown a $4.1 \%$ increase from 2000 to 2010 in the KCP research area and a $3.0 \%$ decline in non-research zones within and outside the national park. Regrowth in the research area relative to further deforestation in the rest of the park demonstrates how long-term field sites can add another consistent layer of habitat preservation. Given that canopy cover has suffered a similar decline in non-research zones inside and outside the park, national park status alone is not a sufficient defence against illegal timber extraction activity. Analyses will be expanded to incorporate other chimpanzee habitat in Uganda under varying levels of protection and research. Research complied with the International Primatological Society Guidelines. 


\title{
Archaeological Investigation of Capuchin Monkey (Sapajus libidinosus) Cashew Processing Sites at Serra da Capivara National Park, Brazil
}

\author{
Michael Haslam ${ }^{a}$, Tiago Falótico ${ }^{a, b}$, Lydia V. Luncz $^{a}$, Eduardo Ottoni $^{b}$
}

aResearch Laboratory for Archaeology and the History of Art, University of Oxford, Oxford, UK; bInstitute of Psychology, University of São Paulo, São Paulo, Brazil

E-Mail: michael.haslam@rlaha.ox.ac.uk

Primate tool use involving resilient materials offers an opportunity to track behaviour back through time, provided tools can be recovered and recognised in archaeological contexts. Here we describe the results of recent survey, mapping and excavation of stone tools used by wild capuchin monkeys (Sapajus libidinosus) in Serra da Capivara National Park, Brazil. We focus on capuchin processing of indigenous cashew nuts, which results in the formation of stone tool concentrations - cashew processing sites - around cashew trees. These tools are recognisable through use-damage, as well as distinct residues coating the tools, which we have characterised using gas chromatography-mass spectrometry. Surface sites consist of hammer and anvil stones preferentially selected and transported from the local natural environment, in addition to use of cashew tree branches and roots as anvil surfaces. Through archaeological excavation we have located buried capuchin cashew processing sites, with hammers and anvils identified through use-wear and their patterning within the site. Our results demonstrate that New World non-human primate behaviour can leave clear and interpretable archaeological signatures.

\section{The Costs of Competition for Male Partners in Wild Female Assamese Macaques}

Christine B. Haunhorst ${ }^{a}$, Michael Heistermann $^{b}$, Oliver Schülke ${ }^{a}$, Julia Ostner ${ }^{a}$

aUniversity of Göttingen, and berman Primate Centre, Göttingen, Germany

E-Mail: c.haunhorst@gmail.com

Female-female competition has been thought to be restricted to resources other than male partners for a long time, except in so called 'sex role-reversed' systems where males provide most or all parental care for the offspring. Recent evidence suggests that females compete for access to males if they provide resources or services enhancing female fitness. Females, therefore, should compete over males that are able to support them and their offspring in agonistic conflicts, or give them some advantage in intra-group feeding competition. We studied two social groups of wild Assamese macaques (Macaca assamensis) in Phu Khieo Wildlife Sanctuary in north-eastern Thailand over three socially stable periods between 2007 and 2013. We found that strong relationships between males and females were predicted by the similarity of their dominance rank in the male and female rank order, which suggests that males or females or both sexes compete for access to the opposite sex. We will investigate in what form females are competing for the same male and evaluate the costs of this competition by comparing faecal glucocorticoid levels of all adult females to assess (1) whether competing females exhibit higher glucocorticoid levels than females with an 'exclusive partner' and (2) whether the dominance rank of competing females influences glucocorticoid levels. Our research complied with the European Directive 2010/63/EU and the International Primatological Society (IPS) Guidelines for the Use of Nonhuman Primates in Research. 


\title{
Considering Alternate Explanations for Paranthropus Mandibular Morphology Using a Primate Comparative Approach
}

\author{
Rebecca Haywood \\ University of Sheffield, Sheffield, UK
}

E-Mail: pra08rkh@sheffield.ac.uk

The highly derived craniofacial morphology of Paranthropus has long been regarded as a specialist dietary adaptation to aide with the consumption of hard foods, and the striking differences in morphology between Paranthropus and Australopithecus interpreted as a reflection of significant differences in the masticatory requirements of diet. However, evidence from stable carbon isotopes and mircrowear analyses challenge this interpretation, and indicate a high percentage of dietary overlap between the two genera. Consequently, other evolutionary scenarios need to be considered to understand potential mechanisms involved in the evolution of Paranthropus mandibular morphology, as well as developing the understanding of the variation observed within Paranthropus and between Paranthropus and Australopithecus. In this study, a series of metric and non-metric variables of the dentition and mandible will be used to describe and compare morphological variation among different Catarrhine and Platyrrhine primate species. This will allow the use of extant primate analogues to evaluate the potential effect of diet and fallback foods, habitat variation and sexual dimorphism as potential underlying processes suggested to affect mandibular morphology. Data from 478 extant primate skeletons were collected from 3 museums. The results of univariate and multivariate analysis suggest that variations in diet and the degree of sexual dimorphism are important factors that affect the mandibular morphology of these non-human primates. These factors will be further analysed in the context of Paranthropus and Australopithecus morphology. Data for this study was gathered from nonhuman primate skeletal collections in accordance with guidelines at each institution. Consequently directives (including the European Directive 2010/63/EU and the IPS Guidelines for the Use of Nonhuman Primates in Research) regarding caring for living non-human primates are not applicable.

\section{Getting Fat or Getting Help? How Female Mammals Cope with Energetic Constraints on Reproduction and Brain Size}

Sandra A. Heldstab, Carel P. van Schaik, Karin Isler

Anthropological Institute and Museum, University of Zurich-Irchel, Zurich, Switzerland E-Mail: sandra.heldstab@uzh.ch

Species living in seasonal habitats experience frequent periods of food scarcity which impose energetic constraints that act most severely on reproductive females. We propose two complementary strategies on how animals cope with periods of food scarcity: species buffer lean periods either by storing fat or by cognitive flexibility in finding alternative resources. As brains are energetically very expensive, but body fat is costly too due to locomotor constraints, we predict a trade-off, expressed in a negative correlation between the ability to store fat and brain size. We further expect that this trade-off between fat storage and brain size is more pronounced in females than in males, since females are more affected by energy constraints due to offspring production. Here, we report a broad phylogenetic comparative study using seasonal variation of body mass as a proxy of the ability to store fat in a sample of 83 mammalian species, including 30 primates. Controlling for phylogeny and body mass, we found a negative correlation between relative brain size and seasonal body mass variation over all lineages. As expected, we also found that this tradeoff was particularly strong in females. However, females may reduce the need to store fat when the costs of reproduction are distributed over several individuals. Thus, an additional influx of 
energy into the mother-offspring unit, as provided e.g. by help of the male or non-breeding group members, is negatively correlated with the amount of body fat in females. These results show that both storing fat and allomaternal energy subsidies independently stabilise the energetic costs for female reproduction in seasonal habitats. In summary, our results support the notion that seasonality plays a role in brain size evolution. Further, they contribute to the understanding of how humans could evolve unusually large brains and at the same time have a larger amount of adipose deposits than expected for a primate of our body size.

\title{
The Self-Organisation of Dominance of Females Relative to Males in Primates
}

Charlotte K. Hemelrijk

University of Groningen, Groningen, The Netherlands

E-Mail: c.k.hemelrijk@rug.nl

The processes that underlie the formation of the dominance hierarchy in a group have long been debated. Models of self-organisation suggest that dominance hierarchies develop by the selfreinforcing effects of winning and losing fights (the so-called winner-loser effect). In addition, dominance hierarchies are influenced by pre-existing individual differences, such as body mass. In the present paper, we investigate the relevance of these two factors for the degree of female dominance over males. We investigate this in a correlative study in which we compare female dominance between groups of 22 species throughout the primate order. In our study, female dominance may range from 0 (no female dominance) to 1 (complete female dominance). As regards effects of body mass, we expected a negative correlation between female dominance over males and species-specific sexual dimorphism in body mass. However, to our surprise we found none (we used the method of independent contrasts). Instead, we confirmed in empirical data two self-organisation hypotheses derived from the winner-loser effect in a computational model: First, that female dominance over males is greater at high than at low intensity of aggression. Second that female dominance increases with the percentage of males in the group. We also show in the model that female dominance increases when males are attracted to them. Since the winner-loser effect has been shown to work in many taxa, including humans, these results may have broad implications.

\section{Origins of Human Cognition and Temperament: A Systematic Comparison of Non-Human Great Apes and Human Children}

\author{
Esther Herrmann
}

Max Planck Institute for Evolutionary Anthropology, Leipzig, Germany

E-Mail: eherrman@eva.mpg.de

Humans differ from their closest living relatives, the great apes, in aspects of cognition and temperament. To understand these differences fully it is important to ask: Do humans possess more complex cognitive skills in general? Or, have humans evolved some specialized cognitive adaptations? And, does human temperament differ from that of other apes in ways which may facilitate the expression of more complex forms of human cognition? To first identify precisely which skills and traits are derived in humans and which are shared with other apes, chimpanzees, orangutans and 2.5-year-old human children were compared on a wide range of physical and social cognitive problems. Our main finding is that human children show similar skills in solving physical cognitive problems to the chimpanzees but already show special social cognitive skills in comparison to other apes. In a second study, we compared children at 3 and 6 years of age with 
chimpanzees on a battery of temperament tasks. Three-year-old children and chimpanzees were very similar in their inhibitory and attentional control skills. Six-year-old children were more skilful than either three-year-olds or chimpanzees at controlling their impulses. These systematic comparisons provide an insight into the origins of uniquely human cognition and temperament and support the idea that humans share many cognitive skills with apes, especially for dealing with the physical world, but that, in addition, they have evolved some specialized social cognition skills. The results further suggest that the origins of human self-regulation lie within our general primate heritage, but that species-unique skills of self-regulation emerge around school age. Research like this stresses our close phylogenetic tie to other great apes and reveals the complexity of great ape cognition. Conservation of the great apes is thus crucial for understanding the peculiarities of our own species and for piecing together our evolutionary history.

\title{
Chimpanzee Tool Use in Northern DR Congo Is Not Tied to Abundance of Insect Prey
}

\author{
T.C. Hicks ${ }^{a, b}$, C. Boesch ${ }^{b}$, H. Kuehl ${ }^{b}$, P. Roessingh ${ }^{c}$, S.B.J. Menken ${ }^{c}$ \\ ${ }^{a}$ Artes Liberales, University of Warsaw, Warsaw, Poland; ${ }^{b}$ Department of Primatology, Max \\ Planck Institute for Evolutionary Anthropology, Leipzig, Germany; Institute for Biodiversity \\ and Ecosystem Dynamics, University of Amsterdam, Amsterdam, The Netherlands \\ E-Mail: clevehicks@ hotmail.com
}

We present the results of a 10 year survey of chimpanzee material culture across a 50,000 $\mathrm{km}^{2}$ region in Northern DR Congo, with a focus on insectivory. We document four kinds of insect foraging tools, as well as an unusual percussive technique used to acquire Cubitermes and Thoracotermes termites, which appears to take the place of Macrotermes fishing, despite the ready availability of Macrotermes mounds in the region. For Dorylus and Pachycondyla ant foraging tools, we investigate whether availability of these insect species at more than a dozen different survey sites influences the likelihood that the chimpanzees will prey on them with tools. Abundance of ant species is not related to the frequency of tool use to prey on them.

\section{Primate Crop Feeding Behaviour and Its Implications for Crop Protection and Conservation: A Stagnant or Burgeoning Field?}

Catherine Hill

Oxford Brookes University, Oxford, UK

E-Mail:cmhill@brookes.ac.uk

Macaques (Macaca spp.), baboons (Papio spp.) and vervet monkeys (Chlorocebus spp.) top the lists of wildlife species that damage crops around African and Asian protected areas. They are thought particularly successful at exploiting crop foraging opportunities because of their social intelligence, dietary and behavioural flexibility, agility and proclivity for semi-terrestrial locomotion. However, many species across a range of primate genera, and irrespective of their dietary and locomotory specializations, can and will incorporate agricultural crops within their diets. There is little doubt that rapid, extensive conversion of natural habitats to agricultural areas is significantly impacting primate populations, but primate crop foraging behaviours cannot be understood solely in terms of animals shifting to cultivated crops to compensate for reduced wild food availability. Consequently, to truly understand why, how and when primates might incorporate crop resources within their dietary repertoire, we need to examine primate crop foraging behaviour within the context of the animals' feeding strategy, i.e., how animals balance the likely 
costs and benefits of adopting such behaviour. In this paper, I briefly summarise what we already know about primate crop foraging behaviour and highlight key areas where systematic data are sparse or absent. I consider the importance of addressing these data deficits, both as a means to further understanding primate capacity to accommodate to anthropogenic change and as a key component to developing effective, humane crop protection methods. Finally, I discuss the importance of recognising and understanding the distinction between developing technical tools to reduce crop losses through primate foraging and mitigating 'human-primate conflict'.

\section{Apes in the Anthropocene: Short-Term Flexibility and Long-Term Survival}

Kimberley Jane Hockings ${ }^{a, b}$

${ }^{a}$ Centre for Research in Anthropology (CRIA-FCSH/UNL), Lisbon, Portugal; ${ }^{b}$ Anthropology

Centre for Conservation, Environment and Development, Oxford Brookes University,

Oxford, UK

E-Mail:hock@fcsh.unl.pt

We are in a new epoch, the Anthropocene, and research into non-human primate behaviour must keep pace with the speed at which our species is driving global change. However, behaviour evinced by primates, including great apes, in human-influenced habitats is sometimes perceived as being less interesting than behavioural patterns exhibited in more 'natural' habitat. In reality, few long-term great ape research sites are unaffected by human influences. Using a case study of chimpanzees inhabiting a forest-farm mosaic at Bossou, Guinea, I provide examples of how chimpanzees evaluate and respond flexibly to challenges posed by humans and their activities. For example, chimpanzees take account of the risks of including agricultural crops in their foraging decision-making and evaluate the risks associated with crossing roads, modifying their behaviour accordingly. I detail how understanding such flexibility can inform research in diverse fields, from cognition to conservation. Despite its importance, our current knowledge of ape flexibility in response to anthropogenic change is scant. Research at those sites especially affected by human activity can provide important templates for predicting the future that likely faces most great ape populations. To ensure that large, diverse ape populations survive the Anthropocene requires finding ways for humans and apes to coexist outside protected areas. Research complied with the European Directive 2010/63/EU and/or the International Primatological Society (IPS) Guidelines for the Use of Nonhuman Primates in Research and adheres to the legal requirements of IBAP and DNRST, Guinea.

\section{The Evolution of Self-Medication in Primates from a Cross Taxa Comparative Perspective}

Michael A. Huffman

Kyoto University Primate Research Institute, Inuyama, Japan

E-Mail: huffman.michael.8n@kyoto-u.ac.jp

Parasites and pathogens cause a variety of diseases that can affect the behaviour and reproductive fitness of an individual and a variety of behavioural responses to parasitism have been reported. Primates have received growing attention for their ability to self-medicate in response to parasite infections. At our current level of understanding, health maintenance and self-medicative behaviours can be classified into four basic levels: (1) optimal avoidance or reduction of the 
possibility for disease transmission (avoidance of faeces contaminated food, water, substrates); (2) the dietary selection of items with a preventative or health maintenance affect (items eaten routinely in small amounts or on a limited basis); (3) ingestion of a substance for the curative treatment or control of a disease; and (4) application of a substance to the body or a living area for the control of disease transmission or a physical condition. Because the need to counteract such pressure is great, anti-parasitic behaviours are expected to occur throughout the animal kingdom. It should be no surprise then that primates are by no means unique in their ability to act in ways that prevent or minimize transmission or control existing parasite infections. Insects utilize the chemical defenses of plants to protect themselves from parasitoid predators and parasites. A number of antiparasitic adaptations found in great apes for example have also been reported among mammals ranging from bats to bears. In this talk, self-medication as an adaptive defense against parasites in primates will be described and compared with similar behaviours in other animal species.

\section{Primates and Bushmeat Hunting Around the High Niger National Park, Guinea, West Africa: Drivers and Patterns of Change}

Tatyana Humle ${ }^{a}$ Alexandre Konate ${ }^{b}$

aDurrell Institute of Conservation and Ecology, School of Anthropology and Conservation, University of Kent, Canterbury, UK; ${ }^{b}$ China Agricultural University, Beijing, Republic of China E-Mail:T.Humle@kent.ac.uk

Bushmeat hunting contributes to the decline of primate species across Africa; however, few studies have explored how such practises change over time in specific localities and how changes in land-use patterns and economic drivers may enhance threats to primates in Muslim dominated areas where, traditionally, primates are spared from such trade. The Haut Niger National Park (HNNP) is one of only two national parks in the Republic of Guinea. The park is one of the last remaining important formations of dry forest-savannah mosaics in West Africa and is a site of high conservation priority for ungulates and the western subspecies of chimpanzee. This study aimed to: (1) estimate the diversity and abundance of animal species sold for consumption across several markets in and around the HNNP, (2) analyse the evolution of the bushmeat trade since the mid-1990s, and (3) identify the players and drivers of the commercial bushmeat trade in the area. Local market assessments were conducted across four village markets and in Faranah, one of the closest urban areas abutting the HNNP. We successfully identified 5,807 wildlife carcasses of 46 species and 22 families on markets surveyed over a 7 month period spanning both the dry and wet seasons. In addition, semi-structured interviews with hunters, farmers and people involved in the bushmeat trade helped identify more recent drivers of the bushmeat trade in the HNNP. Our results indicated an increase in diversity of species targeted and the influence of crop-foraging and local microcredit systems in exacerbating the presence of primate species sold at bushmeat stalls in urban areas. Finally, whilst identifying key recommendations and gaps for future research, this study emphasises the growing risks facing primates as targets for bushmeat where people depend on agriculture and natural resource extraction for subsistence. This study complied with the International Primatological Society (IPS) Guidelines for the Use of Nonhuman Primates in Research. 


\title{
Does More Mean Better? Impact of Two Simultaneous, Different Types of Enrichment (Sensorial and Cognitive) in Chimpanzees (Pan troglodytes)
}

\author{
Noëlla Iglesias $^{a, b}$, Miquel Llorente ${ }^{b, c}$
}

aFundació Universitat de Girona: Innovació i Formació, Girona, bUnitat de Recerca i Etologia, Fundació Mona, Riudellots de la Selva, and IPHES, Institut Català de Paleoecologia Humana i Evolució Social, Tarragona, Spain

E-Mail: Miaigle@hotmail.com

One procedure to increase the well-being of captive animals is the use of enrichment. A few categories exist, for example: food-based, sensory, social and cognitive. It has now been scientifically confirmed that music as a sensory enrichment has a positive effect on the well-being of chimpanzees (Pan troglodytes), as has the use of cognitive enrichment. However, what happens when you combine these two enrichment methods? We studied the effects of a sensorial and a cognitive enrichment (used both separately and simultaneously) within one group of eight chimpanzees and one group of five chimpanzees. Instrumental music was used as the sensorial enrichment, and a tube task (tubes with a double exit, with food inside) was used as the cognitive enrichment (individuals had to use an instrument such as a wooden stick to retrieve reward). Results show that the effect of the two enrichments when used at the same time is not equal to the effects of the two enrichments when separated. Furthermore, it seemed that in these study conditions cognitive enrichment had a stronger effect than sensorial enrichment when they are used simultaneously. At a general level, more positive effects have been found during simultaneous use but negative effects, even if they are not residual, seem to add up too. We conclude that before using the two enrichments together, the end goal has to be clearly defined in order to choose the most suitable way to use them. This research complied with the European Directive 2010/63/EU and the International Primatological Society (IPS) Guidelines for the Use of Nonhuman Primates in Research.

\section{Do Chimpanzees Distinguish between Collaborators and Non-Collaborators after Food Acquisition?}

Maria John ${ }^{a}$, Alica P. Melis ${ }^{a}, b$, Michael Tomasello ${ }^{a}$

${ }^{a}$ Department of Developmental and Comparative Psychology, Max Planck Institute for

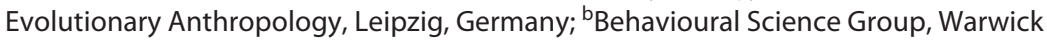
Business School, The University of Warwick, Coventry, UK

E-Mail: maria_john@eva.mpg.de

In 2011, Melis et al. conducted a study investigating the sharing of food after collaborative and individual food acquisition. In that study, two captive chimpanzees were paired and tested on their sharing behaviour after obtaining a monopolizable food object, which the 'captor' (usually the dominant) was able to get either alone or in cooperation with the 'non-captor' (usually the subordinate). It was shown that it was not collaboration that affected the amount of food the non-captor received but instead his proximity to the food at the moment it was acquired by the captor, who was then the limiting factor, denying or granting access to the food. However, chimpanzees are highly social animals and are normally group living. Therefore, we performed a follow-up study to investigate food sharing behaviour in a triad, thus creating a more natural group dynamic. This was done with monopolizable food, which was acquirable by one individual alone (solo condition) or by two individuals in teamwork (collaboration condition). Of special interest were the different proportions of food the collaborator received from the captor compared to the non-collaborator, thus also providing insight into labour evaluation of others in chimpanzees. This study is expected to be a further step in investigating chimpanzees' cognition in regard to 
collaboration and behavioural economy, therefore supporting their image as complex thinking animals. The present study complied with the International Primatological Society (IPS) Guidelines for the Use of Nonhuman Primates in Research.

\title{
Scarcity and Value Attribution in Chimpanzees (Pan troglodytes) and Children
}

Maria John ${ }^{a}$, Federico Rossano ${ }^{a}$, Alica P. Melis ${ }^{a, b}$, Michael Tomasello ${ }^{a}$

aDepartment of Developmental and Comparative Psychology, Max Planck Institute for

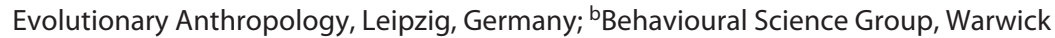
Business School, The University of Warwick, Coventry, UK

E-Mail: maria_john@eva.mpg.de

In order to investigate the phylogenetic and ontogenetic roots of the preference for scarce goods, we performed a study with chimpanzees (Pan troglodytes) as well as 4- and 6-year-old children. We hypothesized that when an object is completely novel to a subject and no other intrinsic information about the article is available, scarce objects will be valued higher than abundant ones and as such should be chosen first. This effect ought to be increased in the presence of competitors. If, on the other hand, a subject is confronted with familiar objects, the amount available will not affect the subject's preferences when the subject is able to choose only one item at a time. Instead, subjects are then expected to make their decision according to predominant preferences. The results of Study 1 suggest that, in chimpanzees, scarcity does not increase the value of a novel object nor does it affect their preferences in relation to familiar objects. The results of Study 2 performed with young human children suggest that they behave like chimpanzees in that scarcity does not affect their preferences independently of familiarity with an object. However, an effect of scarcity in their attribution of higher value to novel objects can be observed in 6-yearold children and is mainly driven by the male participants. These results indicate that the application of the Scarcity Principle is something uniquely human and is likely learned and culturally transmitted. The present study complied with the International Primatological Society (IPS) Guidelines for the Use of Nonhuman Primates in Research.

\author{
My Mother Alison Jolly: Primatologist, Conservationist, Mentor \\ Margaretta Jolly \\ University of Sussex, Falmer, UK \\ E-Mail:m.jolly@sussex.ac.uk
}

Alison Jolly overturned established thinking after becoming the first scientist to do an indepth field study of the behaviour of the ring-tailed lemur, Lemur catta, beginning work in Madagascar in 1962 as a young graduate from Yale. She discovered that this species - and, as it turned out, almost all other lemurs - have female dominance over males, breaking the then orthodoxy that primates were male-dominant. As she later joked, the 'king' of the DreamWorks animation Madagascar ought to have been a queen. My mother saw the ring-tails as pugnacious, swaggering, but also formal to the point of ritual and ever so maternally doting. She herself was not pugnacious at all, though she did indeed dote upon me and her three other children. This presentation will offer a personal perspective on my mother's work, considering the experiences that formed her as a scientist but also what she offered to other colleagues and students as well as her family, as a pioneering woman biologist and conservationist. It will also introduce her final book, Thank You, Madagascar, published posthumously after her death in 2014. Based on diaries writ- 
ten over the decades of her visits to her beloved Madagascar, this book offers a unique, insider's account of a major conservation effort in one of the world's most iconic biodiversity hotspots. But these diaries are also powered by her pleasure in story-telling. As such, they have preserved my mother's voice, and pose a particularly joyful experiment in interpretation for me as a specialist in the scholarly study of feminist life writing.

\title{
Compound Grip in Capuchin Monkeys (Sapajus spp. and Sapajus libidinosus)
}

\author{
C.E. Jones, D. Fragaszy \\ University of Georgia, Athens, Ga., USA \\ E-Mail: cejones@uga.edu
}

Humans routinely handle multiple objects in one hand. Napier (1956) categorized human grips into two varieties: power and precision. Apes, Old World monkeys and capuchin monkeys use both types of grips. MacFarlane and Graziano (2009) defined a compound grip as 'holding an object with many grips simultaneously or holding many separately controlled objects in one hand'. Compound grips have been described in macaques, gorillas and chimpanzees, but not yet in any New World primate. This study examined the ability of seven capuchin monkeys (Sapajus spp.) to execute a compound grip. We predicted, given capuchins' dexterity, including the use of precision grips and analogous neuroanatomy in the motor areas in macaques and capuchins, that these monkeys would use compound grips. Two tasks were designed to elicit compound grips: depositing balls into a small tube, and procurement and release of a combination of small rods and balls on a flat surface. All monkeys held two balls in one hand and dropped them individually into a tube; most could do so with more than two balls. To release several balls, the monkeys put the hand and/or ball in contact with the tube prior to release. During release, monkeys moved a single digit and/or sets of digits laterally and vertically. They picked up rods and balls with one sweeping power grip and deposited them in one releasing motion. Wild capuchin monkeys ( $\mathrm{Sa}$ pajus libidinosus) were filmed more than 100 times holding multiple objects in one hand and occasionally moving the objects within one hand. We conclude that (1) capuchin monkeys hold and move multiple objects in one hand and (2) independent movements of single digits or sets of digits support separate control of objects in one hand. These features meet MacFarlane and Graziano's definition of compound grips. This research complies with the International Primatological Society (IPS) Guidelines for the Use of Nonhuman Primates in Research and UGA IACUC protocol (AUP A2013 10-017-Y2-A1).

\section{Social Network Analysis as a Welfare Indicator: Complete Analysis of Two Groups of Sanctuary Chimpanzees

\author{
Lucía Jorge-Sales $^{a, b}$, Laia Orench $^{a, b}$, Juan Franco-Guzmán ${ }^{a, b}$, Mireia Ruiz-Cabanesa, ${ }^{a}$, \\ Miquel Llorente ${ }^{b, c}$ \\ aFundació Universitat de Girona: Innovació i Formació, Girona, bunitat de Recerca i Etologia, \\ Fundació Mona, Riudellots de la Selva, and CIPHES, Institut Català de Paleoecologia Humana \\ i Evolució Social, Tarragona, Spain \\ E-Mail: luciajorgesales@gmail.com
}

Chimpanzees are highly sociable apes. The different relationships established between chimpanzees during their lives are essential for their development. Taking this into account, social network analysis (SNA) is a suitable theoretical framework to study chimpanzees' relationships. The

6th European Federation for Primatology Meeting
Folia Primatol 2015;86:235-386 DOI: $10.1159 / 000435825$ 
use of SNA has increased in the last decade. Nonetheless, to our knowledge, this is the first time that SNA has been used with sanctuary primates. The sample consisted of 13 chimpanzees housed at Fundació Mona (Spain). They were separated into two distinct groups, one with an invariant structure and the other with a variant composition. In order to define the social structure, we differentiated two networks for each group: grooming and affiliative. These networks were constructed by weighted and asymmetric matrixes. Individual analyses were also included in these networks. UCINET 6.0 was applied for the networks analysis. Welfare was calculated using three indices in order to correlate with the SNA data. Different results were found in the two groups, which did not have the same features. However, results suggest that higher levels of resocialisation are correlated with individual advantageous positions. Regarding our hypothesis, we could conclude that knowing the social position of an individual we could be approximately aware of his welfare. All of this shows that group-housed communities provide opportunities to enhance chimpanzees' welfare. This research complied with the European Directive 2010/63/EU and the International Primatological Society (IPS) Guidelines for the Use of Nonhuman Primates in Research.

\section{Individuality and Anonymity in the Face of Habitat Destruction}

Elfriede Kalcher-Sommersguter ${ }^{a}$, Signe Preuschoft ${ }^{b, c}$

anstitute of Zoology, University of Graz, Graz, and bVIER PFOTEN, Competence Centre Apes, Vienna, Austria; 'Anthropological Institute, University of Zurich, Zurich, Switzerland

E-Mail: elfriede.kalcher@uni-graz.at

A great ape is costly to produce. One offspring takes its mother between 5 (gorilla) and 8 (orang-utan) years of exclusive care before its next sibling is born. Slow life history with extensive and prolonged maternal care, low reproductive rates, slow development with an emphasis on learning, and low spatial densities render great apes particularly susceptible to extinction. The last decades have seen a devastation of the great ape habitats with immense numbers of displaced and dead apes, leaving behind a small fraction of immature offspring rescued into sanctuaries. Yet the numbers of rescued orphans are still large enough to be beyond the capacity of the sanctuaries. There is thus a contrast between the evolved nature of great apes and their massive presence and loss of personal history in sanctuaries due to the extinction crisis. In some sanctuaries, hundreds of apes are held on very limited space, taxing their social capacities and giving rise to unprecedented health risks. Sanctuary staff, normally recruited within the range country, is often motivated by spontaneous compassion but lacks formal caretaker training. No processes are in place to aid these people with burn-out and hopelessness when confronted with the never-ending influx of new orphans, the felling of one area of forest after another and the disappearance, deaths and rumoured demise of their former charges after release. However, where professional individualised care can be afforded, the recovery of even severely mutilated apes is spectacular. 


\title{
Early Traumatic Experience Has Lifelong Effects on Social Integration of Chimpanzees
}

Elfriede Kalcher-Sommersguter ${ }^{a}$, Signe Preuschoft ${ }^{b}$, Cornelia Franz-Schaider $^{a}$, Karl Crailsheim $^{a}$, Jorg J.M. Massen ${ }^{c, d}$

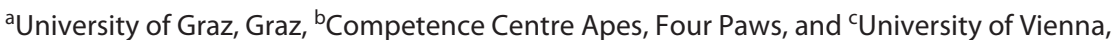
Vienna, Austria; ${ }^{d}$ University of Utrecht, Utrecht, The Netherlands

E-Mail: elfriede.kalcher@uni-graz.at

Enduring adverse early rearing conditions is shown to affect the social behaviour of human and non-human primates. Contrary to humans, however, information regarding the long-term effects of early trauma, such as loss of the mother, is so far sparse for non-human primates. Therefore, we investigated the impact of early trauma in chimpanzees on their later social integration into a group. In particular, we focused on the effects of age at onset as well as duration of trauma/ deprivation on later social integration using social network analysis (SNA), i.e. we calculated individual network measures, and compared these measures of adult early (EDs, $n=10$ ) and late deprived (LDs, $\mathrm{n}=8$ ) ex-laboratory chimpanzees living in three social groups subsequent to resocialization, as well as during their second year of group-life, to those of adult zoo chimpanzees living in two stable groups, consisting of non-deprived (NDs, $\mathrm{n}=16$ ) and wild-caught maternally deprived individuals (MDs, $n=9$ ). We focused on SNA-measures regarding the tolerance of close proximity and social grooming as we expected these behaviours to be severely affected by breaking the mother-infant bond in early infancy. Our study revealed, that the tolerance of conspecifics close-by was most strongly affected by the duration of deprivation as EDs and LDs were much more selective regarding their association partners subsequent to re-socialization than were MDs and NDs. However, EDs and LDs partially recovered during the second year of group-life. In contrast, grooming behaviour seemed more affected by age of onset of trauma, as grooming activity of EDs and MDs, but not of LDs, was significantly lower and much more selective compared to that of NDs. Thus, our data suggest that, as in humans, early traumatic experience and lack of social experience strongly affect social integration of chimpanzees throughout their lifetime; made particularly notable since our MD chimpanzees had sometimes had more than 40 years to recover.

\section{Primate Quadrupedal Walk Evolution: Lessons from the Diagonal Gaits of the Feathertail Marsupial Glider Acrobates pygmaeus (Acrobatidae, Diprotodontia)}

\author{
Nikolaos Evangelos Karantanis ${ }^{a}$, Dionisios Youlatos ${ }^{a}$, Leszek Rychlik ${ }^{b}$
}

aDepartment of Zoology, School of Biology, Aristotle University of Thessaloniki, Thessaloniki, Greece; ${ }^{b}$ Department of Systematic Zoology, Institute of Environmental Biology, Faculty of Biology, Adam Mickiewicz University, Poznań, Poland E-Mail: nekarantanis@gmail.com

Research on primate origins has been revolving around arboreality, and more specifically the adaptations that are linked to safe navigation in the fine-branch niche. To this end, extant non-primate mammals have been used as models to assess the significance of these adaptations. However, the size of these models is usually larger than that estimated for early primates. The current study proposes an alternative, very small-bodied model that can simulate better early primate representatives. The feathertail marsupial glider Acrobates pygmaeus, with a body mass of $12 \mathrm{~g}$, a clawless opposable hallux and terminal branch feeding habits, appears more suited to modelling behavioural adaptations to the small branch milieu. Analysis of video recordings of 18 captive feathertail gliders walking on poles of variable diameter and inclination revealed that they 
preferentially used diagonal gaits more often, especially on smaller substrates and during inclined ascents. Duty factor indices correlated with diagonality in higher velocities, and increased from descents to horizontal to ascents. Moreover, stride length played a minor but significant role in velocity regulation, and velocity changes were mainly coordinated by stride frequency on all substrate categories regardless of direction of movement. The current study shows that tiny arboreal marsupials exhibit some of the behavioural features that characterize primate quadrupedalism. Furthermore, it implies that these features may have been favourable under unstable branch conditions for small arborealists. This highlights the importance of small body size in early primate evolution, which could have provided the selective advantage for navigating within a fine branch niche. The present study complies with Greek and Polish laws for the use of captive animals in research and the European Directive 2010/63/EU.

\section{Enhancing the Profile of Threatened Primates: Impact of 'Top-25' Listing on the Choice of Species for Scientific Study}

Daphne Kerhoas ${ }^{a}$, Alberto Acerbi $^{b}$, Amanda Webber $^{a}$, Christoph Schwitzer ${ }^{a}$

aBristol Zoological Society, and bepartment of Archaeology and Anthropology, University of Bristol, Bristol, UK

E-Mail: daphnekerhoas@gmail.com

A list of the World's 25 Most Endangered Primates has been decided by consensus biennially since 2000 in order to draw attention and action towards a few species that are considered to be amongst the most endangered primates worldwide. We investigated, for the first time, whether the inclusion of a species in the Top-25 list had an influence on the number of peer reviewed articles published on that species in the following years. This is of vital importance because policy-makers and funding agencies rely mostly on scientific reports. Therefore, an increase in published literature about the most endangered primate species would promote action to save them. We performed text-mining of the metadata made available by several major publishers (namely PLoS, BMC, Elsevier, Springer, Nature and Highwire/Pubmed), analysing a total of about 40 million articles. We extracted articles where the name of a species present in any of the seven Top-25 lists published since 2000 appeared at least once ( $n=69$ species, found in 7,469 articles), and where the name of a primate control species (never included in the Top-25 list) appeared at least once ( $\mathrm{n}=85$ species, found in 13,656 articles). We calculated the trend per species during the years preceding the mention and we compared it with the trend during the years following the mention, over a time lag of 2, 3 and 5 years. According to our results, 2 years is a too short period to see any effect of a mention in the Top- 25 list. However, within 3 or 5 years of a species being mentioned in the Top-25 list, there is an increase in the scientific output on these species, compared with the control species never mentioned in the list. In conclusion, the inclusion of a species in the Top-25 has a positive effect on the scientific output. 


\title{
Monitoring Spatio-Temporal Patterns of Resource Availability and Acquisition of Spatial Representations of Food Resources in Humans (Homo sapiens) Tested in a Virtual Reality Foraging Task
}

\author{
Melissa Kirby, Carlo De Lillo \\ University of Leicester, Leicester, UK
}

E-Mail: mk217@le.ac.uk

Field studies have suggested that chimpanzees use fruiting synchrony information, and acquire mental representations of the locations of fruiting trees, when foraging in forest environments. This set of experiments aimed to identify the cognitive abilities deployed by humans in a virtual reality foraging task. The task was designed to capture essential aspects of foraging behaviour observed in chimpanzees which is difficult to experimentally assess effectively with nonhuman primates. Participants, with an age range of 18-34, navigated through arrays of coloured poles representing tree species and checked them for the presence of food items. Experiment 1 $(\mathrm{n}=20)$ and $2(\mathrm{n}=24)$ assessed the ability to monitor one or two fruiting patterns across trials simulating the ephemeral ripening of fruit in the forest canopy. Participants were presented with either predictable or unpredictable patterns of food availability. Experiment $3(n=18)$ assessed whether humans acquire spatial knowledge during foraging tasks where visual cues may overshadow spatial ones. It featured only a single temporal pattern in a training phase, before removing the informative value of colour in a transfer phase. Results showed that in predictable conditions, participants monitored multiple patterns of fruit availability and directed searches at the outset of a foraging bout to locations that were most likely to yield fruit. When visual cues were removed, searches to correct locations at the outset of trials were significantly above chance level and searches in locations that never yielded fruit were significantly below chance level. These findings provide support for the assumptions made by field researchers about the cognitive abilities required during foraging in primate species. Results are discussed in relation to the cognitive demands imposed by foraging for ephemeral resources and the notion that they may have triggered the expansion of brain capacity in primates.

\section{Who Fights and Who Wins? Intergroup Relationships in Verreaux's Sifakas (Propithecus verreauxi) \\ Flávia Koch ${ }^{a}$, Claudia Fichtel ${ }^{a}$, Johannes Signer ${ }^{b}$ \\ aDeutsches Primatenzentrum (DPZ), and bUniversity of Göttingen, Göttingen, Germany E-Mail: biokoch@gmail.com}

How animals achieve collective action for territorial defence between neighbouring groups is a central topic in animal behaviour. Since intergroup conflicts are costly, individuals should participate in encounters when the benefits overcome the costs. Members of a group can have different interests and constraints regarding conflicts, which may explain different patterns of individual participation in intergroup encounters. Different factors can also contribute to the power of a group and therefore its chances to win a dispute, such as group size and location of the conflict. In order to examine factors influencing individual participation and outcome in intergroup encounters, we observed the interactions between 8 groups of Verreaux's sifakas in western Madagascar. Our results are based on direct observations of encounters, and on encounters inferred from remote data from GPS loggers present in each group in the study. Male sifakas participated more often in encounters than females. Males and females were more likely to join conflicts when their own group had more males than the opponent group. In sifakas, groups with more males were more likely to win conflicts, therefore male participation might be influenced 
by achieving a numeric advantage over the opponent. In addition, females participated less often in conflicts when dependent infants were present. Thus, in sifakas, male and female participation in intergroup conflicts is mainly influenced by the potential power of the opponent group. A spatial analysis of the location of intergroup encounters revealed that 'prior residency status' did not influence the outcome of a conflict. However, our results indicate that one of the benefits of winning a conflict is the possibility to stay in the area of the encounter afterwards, independent of the 'prior-residence status' of opponents. Thus, in sifakas, winning groups benefit by a 'postresidence status', which may influence the motivation to fight over a given area.

\section{The Acoustic Niche of the Mozambique Dwarf Galago (Galagoides granti) in Southern Africa}

Nokuthula Kom, Fabien Génin, Judith Masters

African Primate Initiative for Ecology and Speciation (APIES), Department of Zoology and Entomology, University of Fort Hare, Alice, South Africa

E-Mail:200908211@ufh.ac.za

Animals may compete for acoustic space (acoustic niche) in the same way they do for ecological space, but interspecific competition, to be a directional force, requires interspecific recognition. For instance, the rarity of the Mozambique dwarf galago (Galagoides granti) in South Africa may be explained by ecological dominance of the larger thick-tailed greater bushbaby (Otolemur crassicaudatus). Alternatively, ecological partitioning also predicts a difference in body size, but no niche overlap. Two nocturnal and one diurnal surveys were conducted recording calls of the two bushbaby species for $5 \mathrm{~min}$ every $100 \mathrm{~m}$, along pre-existing trails. Despite significant overlap in habitat, diet and frequency overlap in their vocal repertoire, no instance of contest or response to interspecific calls was observed. The rarity of Galagoides granti is more likely to be the result of the rarity of its preferred habitat (a complex mosaic of forest and woodland) than competition with Otolemur crassicaudatus.

\section{Feast or Famine: An Investigation of Scarcity and Co-Feeding Effects in Chimpanzees}

Rebecca Koomen, Esther Herrmann

Department of Developmental and Comparative Psychology, Max Planck Institute for Evolutionary Anthropology, Leipzig, Germany

E-Mail: rebecca_koomen@eva.mpg.de

Competition over limited resources is a ubiquitous challenge to overcome in the lives of humans and other primates alike. Competition can be influenced by various characteristics of the resource itself, such as abundance or scarcity, as well as the social and ecological environment in which such goods are acquired (e.g. Gardner, Ostrom, \& Walker, 1990). However, we know relatively little about the behavioural variation in humans' closest relatives, the chimpanzees, who face competition over limited resources while foraging together, for example, on patches of ripe fruits in the wild. If a patch is scarce, do their feeding patterns differ from when the patch is abundant? Does competition over the communally accessible fruit play a role over-and-above simple social facilitation? Here, we addressed these questions by varying the abundance of a food resource when two chimpanzees were either competing over it, or feeding on a pre-divided resource next to one another. Chimpanzees were presented with an apparatus comprising a matrix of 36 
horizontal, transparent tubes which, in the competitive condition, could be accessed by both chimpanzees. In the non-competitive control condition each chimpanzee could access only half of all tubes, such that the resource was pre-divided into two equal, visible shares. In the abundant condition, all 36 tubes were baited, whereas only 8 tubes were baited in the scarce condition. Results show that the social context plays a significantly more important role in determining chimpanzee feeding rate than abundance of the food resource itself, which had no effect on feeding rate. As predicted, the mere presence of a partner engaged in the same feeding context increased feeding rate from baseline, corroborating previous studies on social facilitation in chimpanzees (Engelmann, Herrmann, \& Tomasello, submitted). Direct competition, however, resulted in an increase of feeding rate significantly larger than partner presence alone. This research project complied with the European Directive 2010/63/EU and the International Primatological Society

(IPS) Guidelines for the Use of Nonhuman Primates in Research.

\title{
Sex Differences in Object Manipulation in Immature Chimpanzees and Bonobos: Preparation for What?
}

\author{
Kathelijne Koops ${ }^{a, b}$, Takeshi Furuichic, Chie Hashimotoc, Carel P. van Schaik ${ }^{a}$ \\ aUniversity of Zurich, Zurich, Switzerland; ' University of Cambridge, Cambridge, UK; \\ ‘Kyoto University, Inuyama, Japan \\ E-Mail: Kathelijne.koops@uzh.ch
}

Sex differences in immatures predict behavioural differences in adulthood in many mammal species. Because most studies have focused on sex differences in social interactions, little is known about possible sex differences in 'preparation' for adult life with regards to tool use skills. We investigated sex differences in object manipulation in immature apes. Chimpanzees use various tools across a range of contexts, whereas bonobos use few tools and none in foraging. In both species, a female bias in adult tool use has been reported. We studied object manipulation in immature chimpanzees at Kalinzu (Uganda) and bonobos at Wamba (DR Congo). Chimpanzees showed higher rates and more diverse manipulation types than bonobos. Against expectation, male chimpanzees showed higher object manipulation rates than females, whereas in bonobos no sex difference was found. However, object manipulation by male chimpanzees was play-dominated, whereas manipulation by female chimpanzees was more diverse (e.g. break, bite). Manipulation by younger immatures ( $<3$ years) in both species was also dominated by play, but in chimpanzees became more diverse in older immatures ( $>3$ years). In chimpanzees, object types manipulated also became more tool-like (i.e. sticks) with age. The male bias in object manipulation in immature chimpanzees, along with the late onset of tool-like object manipulation, indicates that not all object manipulation (i.e. object play) prepares for tool use. Instead, given the similarity with gender differences in human children, it may also function in motor skill practice for male-specific behaviours (e.g. displays). In conclusion, even though immature behaviours almost certainly reflect preparation for adult roles, more detailed future work in multiple contexts is needed to disentangle possible functions. The research complies with the European Directive 2010/63/EU and the International Primatological Society (IPS) Guidelines for Use of Nonhuman Primates in Research. 


\title{
Revisiting Animal Empathy: What Are We Looking for?
}

\author{
Sonja E. Koski
}

Centre of Excellence in Research on Intersubjectivity in Interaction, University of Helsinki, Helsinki, Finland

E-Mail: sonja.koski@helsinki.fi

Empathy, the ability to share and represent others' emotional states, is the fundamental feature of human interaction, as it forms the foundation of our intersubjectivity. Intersubjectivity, defined as mutual understanding of another's subjective experience, is the core process in human interactions. Nevertheless, the evolution of empathy remains elusive due to the difficulties of assessing its presence in non-humans. Here, I discuss approaches towards and evidence for primate empathy, aiming to develop an operational framework for advancing the research into non-human animal empathy. The framework is adopted from human studies, which lay out a developmental scheme for empathy and subsequent prosocial behaviour. Intriguingly, recent evidence suggests the existence of other-oriented concern with limited cognitive representational abilities, earlier than thus far believed, emphasizing the importance of intersubjective motivation and selfregulation of emotions. Comparative evidence in non-human primates is relatively scarce, mainly relying on prosocial behaviour rather than the empathic process as such. Therefore, I focus especially on the emotional, cognitive and self-regulatory processes underlying the empathic response and formulate a framework for addressing the details of empathy in primates. Specifically, I suggest that it is crucial to address the degree and valence of emotional contagion, the regulation of self-oriented distress, and the representational abilities allowing cognitive appraisal of another's emotional state. While challenging, it remains crucial to also address the various motivational, emotional and cognitive processes in prosocial behaviour. The research complies with the European Directive 2010/63/EU and the International Primatological Society (IPS) Guidelines for the Use of Nonhuman Primates in Research.

\section{Does Personality Affect the Performance of Abnormal Behaviour and the Reaction to Intervention in Captive Chimpanzees?}

Godelieve Kranendonk ${ }^{a}, b$, Arjan de Klepper ${ }^{a}$, Hester van Bolhuis ${ }^{a}$, Martin Brüne ${ }^{c}$, Berry Spruijt ${ }^{b}$

${ }^{a} A A P$, Rescue Centre for Exotic Animals, Almere, and ${ }^{b}$ Animal Ecology, Department of Biology, Utrecht University, Utrecht, The Netherlands; ' Research Department of Cognitive Neuropsychiatry and Psychiatric Preventive Medicine, LWL-University Hospital, Bochum University, Bochum, Germany

E-Mail: godelieve.kranendonk@aap.nl

Captive chimpanzees are likely to develop abnormal behaviour. Whether or not they actually do develop abnormal behaviour depends on many factors. One of these factors may be the personality of the individual chimpanzee, with personality being the expression of behavioural patterns consistent over time and context. In humans, behavioural disorders are often linked with certain personality traits. In the current preliminary study, 17 captive chimpanzees were observed, using focal animal sampling and continuous recording. Their personalities were analysed and effects on the performance of abnormal behaviour were assessed. Preliminary analyses confirmed that personality of the chimpanzees consisted of five traits (i.e. Social Index, Affection Index, Proximity, Anxiety, and Activity). Preliminary data indicated that two of these five traits, namely Social Index and Activity, affected the performance of abnormal behaviour. Averagely social chimpanzees and less active chimpanzees were found to perform more abnormal behaviour compared to respectively more social and more active chimpanzees. At a later stage, the 
chimpanzees were submitted to an intervention to reduce abnormal behaviour. Analyses will be presented that will show whether or not chimpanzees with a different personality were affected differently by this intervention.

\section{Ontogeny and Variability of Play Behaviour in Wild Bornean and Sumatran Orangutans}

Julia Kunz ${ }^{a}$, Sonja Falkner ${ }^{a}$, Caroline Schuppli ${ }^{a}$, Anna Marzec ${ }^{a}$, S. Suci Utami Atmoko ${ }^{b}$, Carel van Schaik ${ }^{a}$, Maria van Noordwijk ${ }^{a}$

${ }^{a}$ Anthropological Institute and Museum, University of Zurich, Zurich, Switzerland;

bUniversitas Nasional Jakarta, Jakarta, Indonesia

E-Mail: juliaandrea.kunz@uzh.ch

According to some hypotheses, play serves different purposes at different times during ontogeny and therefore the nature and frequency of play are expected to change during the developmental trajectory. With their extended immature phase and ecological and behavioural variation among populations and species, orangutans offer the opportunity to disentangle specific functions of different types of social and solitary play. In this study, frequency and qualitative characteristics of play were compared across two study populations ( $P$. pygmaeus wurmbii, at Tuanan, Borneo, and P. abelii at Suaq Balimbing, Sumatra), ages and sexes in a variety of contexts. During full-day focal follows, play behaviour of immature orangutans (0.3-13 years) was documented by instantaneous and all-occurrence sampling at the two sites $(>5,000 \mathrm{~h}$ long-term activity data per $2 \mathrm{~min}$, of which $>1,000 \mathrm{~h}$ were of very detailed data). We found different ontogenetic trajectories of solitary locomotor, solitary object and social play. While solitary play frequency and quality did not differ across sites, immatures at Suaq Balimbing spent more time in social play than at Tuanan, and played with more different partners. This difference can be explained by the higher degree of sociability and hence, increased social play opportunities, rather than a difference in the intrinsic motivation to play. Indeed, when in association, immatures at Tuanan tended to play even more than those at Suaq Balimbing. Nonetheless, neither social nor solitary play frequencies were affected by fruit availability at either site, suggesting animals must maintain a minimum level of social play regardless of the costs. Because different play types follow different, fixed developmental trajectories in orangutans and happen irrespective of the ecological and social circumstances and details, play probably is a crucial element during the ontogeny of orangutans.

\section{To Be or Not to Be a Vocal Learner}

\section{Adriano R. Lameira}

Department of Anthropology, Durham University, Durham, UK; Pongo Foundation, Oudewater, The Netherlands

E-Mail: adriano@orangutan.nl

Current hypotheses on language evolution propose vocal learning - the capacity to learn new calls by modification or imitation - as the behavioural and cognitive spark for the emergence of speech and human song. There is, however, little evidence to date for vocal learning in non-human primates, including great apes, a position that has caused controversy and that directly challenges the importance of shared ancestry as stated in Darwin's theory of natural selection. Here I present two cases of vocal learning in ex-entertainment orangutans. The first case describes the observation of new voiced and voiceless call production at a speech-like rhythm ( 5 open-close lip cycles per second), a putative instance of homology with human 
consonants and vowels in terms of articulation, acoustics, rhythm and acquisition. The second case empirically demonstrates that an orangutan is capable of real-time (i.e. instantaneous) and dynamic (i.e. alternating modulation of pitch up- and downwards along the frequency spectrum) vocal fold control in order to vocally match human vocal demonstrations produced randomly; this indicates that great apes possess a latent capacity for the deployment of vocal fold oscillation which is qualitatively similar to that exhibited in human intonation and signing in terms of tempo and motoric plasticity. An important step for an advanced theory of language evolution will involve determining the contexts and motivational drives that compel great apes to attend to and learn new vocal signals from humans. This research complied with the International Primatological Society (IPS) Guidelines for the Use of Nonhuman Primates in Research.

\section{Object Manipulation and Tool Use in Wild Chimpanzees of the Budongo Forest, Uganda}

Noemie Lamon ${ }^{a, b}$, Klaus Zuberbühler ${ }^{a}$

aUniversity of Neuchâtel, Neuchâtel, Switzerland; bBudongo Conservation Field Station, Masindi, Uganda

E-Mail: noemie.lamon@unine.ch

Decades of research show that wild chimpanzees have the greatest frequency, diversity and complexity of tool behaviour of any non-human animals. The tool-using behaviours of wild chimpanzees vary across populations in tool forms, materials used and functions, with the last not only related to foraging, but serving in a variety of behavioural contexts. The Sonso community of the Budongo Forest, Uganda, is an interesting case because individuals manufacture and use only one tool in the feeding context, the leaf-sponge, and the recently observed and studied moss sponge. Remarkably, and without any clear ecological or genetic explanation, no Sonso chimpanzee has ever been observed using a stick, a widely used tool in many other East and West African communities. However, they have developed an important leaf technology, which is mainly used in body care but also to extract water and in social interactions. The main purpose of my research is to understand the factors that have led to the proliferation of the Sonso leaf technology and the striking absence of any stick-based tool use behaviour. A first line of investigation is ontogenetic and consists in documenting when and how, during their development, the materials commonly used for tools by adult chimpanzees obtain and lose their salience as play objects to individuals of various age classes. To this end, I have studied in detail object manipulations in all age and sex groups. A second line of research consists of an exhaustive documentation of the leaf technology of this community, by generating a comprehensive list of their tool use.

\section{Subadult Females as Social 'Brokers' in Olive Baboons (Papio anubis): Effects on Social Cohesion}

\section{Julia Lehmann, Piotr Fedurek}

University of Roehampton, London, UK

E-Mail: j.lehmann@ roehampton.ac.uk

Most studies of primate social networks are based solely on adult individuals; subadults are occasionally included but juveniles very rarely so. In this study, we investigated how the exclusion of juveniles from social network analysis affects the conclusions drawn about the social roles 
and positions of different age-sex classes. Based on three months of behavioural data from one troop of wild, habituated olive baboons in Gashaka-Gumti National Park, Nigeria, we constructed three different social networks (grooming, affiliation and aggression). We then used knockout simulations to assess how juveniles affected the apparent social positions of adult and subadult females. We found that juveniles contributed significantly to the social structure of the group. Excluding them from social networks had a particular effect on our understanding of the social role of sub-adult females. It emerged that sub-adult females act as brokers between juveniles and adults, and as such are important for social network cohesion. Their distinct social position became clear only when juveniles were included into the social networks, and was masked when they were excluded. The effects of incomplete networks on our understanding of primate social roles will be discussed. Our research fully complied with the European Directive 2010/63/EU and the International Primatological Society Guidelines for the Use of Nonhuman Primates in Research.

\title{
Explorative 3D Geometric Morphometrics on Crania and Mandibles of Cercopithecini $(1,024$ Specimens)
}

\author{
Stefania Lo Bianco, Luca Sineo \\ Università degli Studi di Palermo, Palermo, Italy \\ E-Mail: stefania.lobianco01@unipa.it
}

A dataset of crania and mandibles from Old World primates was investigated through the use of 3D Geometric Morphometrics. We focused attention on the tribe Cercopithecini, sampling 1,024 specimens preserved in 9 different museums and relating to a total of 31 species. Another 102 specimens belonging to 10 species of the same family, Cercopithecidae, were added as outgroup. Our sampling was carried out using a 3D-digitizer Microscribe G2X and the data were processed through the softwares Morphologika and MorphoJ. We conducted both PCA and CVA analyses to investigate the power of resolution of the morphometric method and to understand the role of a-priori groups in the discrimination of the samples. Variables, such as sex, age and geographic localization, where present, were added to the analyses. Different sets of landmarks were selected with the aim of highlighting those characters with major variability. Even if every skull showed a peculiar morphology, we were not able to go beyond the genus level for genera with many species within it. We found that the a-priori specification is fundamental to obtain a clear clustering of genera. The 'sex' variable did not affect the reliability of the analyses, probably because factors we did not consider, like body and teeth size, have a strong impact on discrimination. With regard to the 'age' variable, 'infant' samples clustered separately from 'adult' ones, while 'juveniles' lay in the middle. Surprisingly, we found a slight split between West and East African species. Morphometric methodology can be helpful in the separation of species with deep morphometric differences or long evolutionary distances. Nevertheless, as in the tribe Cercopithecini, where most of the species show a recent history and similar lifestyle, it was not possible to discriminate up to the species level with this method. Thus, we consider it more useful to adopt a genus level approach to study the evolutionary history of the tribe. 


\section{The Prospective Use of Occupancy Modelling as a Tool for Monitoring Potto (Perodicticus ibeanus) Populations in Kibale National Forest, Uganda}

Averee Luhrs, S.K. Bearder, K.A.I. Nekaris

Nocturnal Primate Research Group, Oxford Brookes University, Oxford, UK

E-Mail: averee.m.I@gmail.com

Nocturnal strepsirrhines are some of the world's most under-studied primates. The potto (Perodicticus spp.) is no exception. Pottos are thought to have a wide range across Central Africa, but evidence is largely anecdotal and outdated. In this study, we are first compiling a comprehensive list of previous potto sightings across Africa. We will then create a GIS map with a Maxent model of potto range according to our unpublished data and the available literature. This will highlight priorities for future research. Secondly, we will test the use of occupancy modelling as a tool for monitoring the population of the eastern potto (Perodicticus ibeanus), Thomas' dwarf galago (Galagoides thomasii), and the lesser needle-clawed galago (G. matschiei) in Kibale National Forest (KNF), Uganda. We have already confirmed the presence of all three of these lorisiforms in KNF during surveys conducted in 1998 and 2006. We will collect presenceabsence data using fixed-point sampling of at least 30 points throughout the park. The results of this study will shed new light on the distribution of nocturnal primates in Kibale National Park. If occupancy modelling proves to be a useful method for accurately monitoring cryptic primate populations, it may be easily replicated across the habitats of African lorisiforms. Currently most African lorisiform species are considered Data Deficient or Least Concern on the IUCN Red List; a method such as occupancy modelling, which also takes into account detectability in various habitats, will help to improve predictive models to gain a better understanding of these nocturnal primates across their wide distribution range. A simple method for monitoring the nocturnal primate population could be integrated into the long-term primate research that characterizes Kibale National Park. No direct contact with animals will occur, and all research will follow the International Primatological Society (IPS) Guidelines for the Use of Nonhuman Primates in Research.

\section{Seasonal Variation of Food Properties Influences Tool Selection in Wild Capuchin Monkeys (Sapajus libidinosus) at Serra da Capivara National Park, Brazil}

Lydia V. Luncz ${ }^{a}$, Tiago Falótico ${ }^{a, b}$, Eduardo Ottoni $^{b}$, Michael Haslam $^{a}$

aResearch Laboratory for Archaeology and the History of Art, University of Oxford, Oxford, UK; ' 'Institute of Psychology, University of São Paulo, São Paulo, Brazil

E-Mail: lydia.luncz@rlaha.ox.ac.uk

In the Tai forest of the Côte d'Ivoire, some wild western chimpanzee (Pan troglodytes verus) communities adjust their tool selection to the properties of processed nuts. At the beginning of the fruiting season, when nuts are harder to crack, they use more stone tools. At the end of the season, when nuts are dry and easier to crack, they use an elevated number of wooden tools. We therefore hypothesised that other stone-tool using primates may adjust their tool use behaviour to changing food properties during a fruiting season. We tested if wild capuchin monkeys (Sapajus libidinosus) from a group in Serra da Capivara National Park adapt their tool selection according to cashew nut properties. Cashews are indigenous to the area and they contain caustic liquid in their shells which is more easily spread when nuts are fresh. The monkeys use stone tools to open both fresh and dry nuts as part of their natural foraging behaviour. We found that the nuts were less resistant and easier to crack towards the end of the fruiting season, when they are dry. 
The capuchins used significantly larger stones to process the fresh nuts, and males selected larger (and therefore heavier) stone tools than females. For both fresh and dry nuts, the number of hits used to open a nut was influenced only by the hammer size (fewer hits with larger hammers). We demonstrate that, as for some chimpanzees, a wild capuchin group also adjusts their tool use behaviour to changing food properties, suggesting that durable tool types and properties can act as a guide to past behaviour for stone-tool using primates. This research complied with the International Primatological Society (IPS) Guidelines for the Use of Nonhuman Primates in Research and had the previous approval of the Brazilian environmental agency (IBAMA - 37615-2).

\section{Male Quality, Dominance Rank, and Mating Success among Free-Ranging Rhesus Macaques}

Dario Maestripieri, Alexander Georgiev

University of Chicago, Chicago, III., USA

E-Mail: dario@uchicago.edu

In this study we investigated the relationship between physiological indicators of body condition (or 'quality'), dominance rank and mating success among 15 adult males in the free-ranging rhesus macaque (Macaca mulatta) population on Cayo Santiago, Puerto Rico. We assessed male condition with two measures of innate immune function (bacteria killing assay and haemolytic complement assay) and one measure of oxidative status (lipid peroxidation). There were significant differences in condition in relation to rank, with high-ranking males having more robust immune response and lower oxidative damage than lower-ranking males. Our measures of male quality were also correlated with mating success, but these correlations became nonsignificant after rank was controlled for in the analyses. Our results suggest that high rank is associated with better condition even in a primate species in which obtaining alpha status does not depend on a male's direct competitive ability but on the amount of time spent in a group (i.e., seniority).

\section{Environmental Factors Affecting Energetic Status in Wild Barbary Macaques \\ Laëtitia Maréchal $^{a, b}$, Stuart Semple ${ }^{a}$, Bonaventura Majolo ${ }^{b}$, Ann MacLarnon $^{a}$ \\ aUniversity of Roehampton, London, and bUniversity of Lincoln, Lincoln, UK \\ E-Mail: LMarechal@lincoln.ac.uk}

Energetic status is a crucial factor for animal survival and reproduction. The recent development of methods for assessment of urinary C-peptide and faecal glucocorticoid measures in wild primates provides powerful tools for non-invasively assessing energetic condition among these animals. While a number of published studies to date have used these techniques to investigate seasonal and/or ecological variation in energetic balance in wild primates, none has done so in species that experience very marked climatic variation using two different hormonal measures related to energetic condition. In this study, we investigate the energetic condition of wild Barbary macaques (Macaca sylvanus) in the Middle Atlas Mountains, Morocco. This environment is highly seasonal, with temperature ranging from $35^{\circ} \mathrm{C}$ in summer to $-10^{\circ} \mathrm{C}$ in winter. We collected data on two troops in the same geographical area, one provisioned by tourists and the other relying on natural food. This system provides an ideal model to investigate how energetic status may be related to environmental variation and how provisioning may buffer animals 
against such variation. Evidence was found in the present study to support the idea that urinary C-peptide and faecal glucocorticoid levels may be related to seasonal and/or ecological variation. The study complied with the European Directive 2010/63/EU and/or the International Primatological Society (IPS) Guidelines for the Use of Nonhuman Primates in Research and, the research was submitted for ethics consideration under the reference LSC 15/ 124 in the Department of Life Sciences and was approved under the procedures of the University of Roehampton's Ethics Committee.

\title{
Do Baboons Show a Language-Like Cerebral Asymmetry? An in vivo Anatomical MRI Study of the Planum Temporale in 90 Subjects
}

\author{
Damien Marie ${ }^{a, b}$, Muriel Roth ${ }^{c}$, Romain Lacoste ${ }^{b}$, Bruno Nazarian $^{c}$, Alice Bertello $^{a, b, d}$,
} William D. Hopkins ${ }^{e, f}$, Jean-Luc Anton ${ }^{c}$, Adrien Meguerditchian ${ }^{a, b}$

aLaboratoire de Psychologie Cognitive, UMR7290, Université Aix-Marseille/CNRS, Marseille, bStation de Primatologie CNRS, UPS846, Rousset, Institut des Neurosciences de la Timone, UMR7289, Université Aix-Marseille/CNRS, Marseille, and dEcole Nationale Vétérinaire, Toulouse, France; ${ }^{\text {eThe }}$ Yerkes National Primate Research Center, Emory University, and fNeuroscience Institute and the Language Research Center, Georgia State University, Atlanta, Ga., USA

E-Mail: m.damienmarie@gmail.com

It has been acknowledged that human show a functional left hemispheric specialization (HS) for language, most of the linguistic functions being mainly controlled by the left hemisphere. Many studies reported that the planum temporale (PT) is on average larger on the left hemisphere than on the right. Interestingly, this anatomical asymmetry is positively correlated with the functional leftward asymmetry of the language networks. With regard to the phylogenetic proximity of human and non-human primates (NHP), exploring asymmetries of the communication system in NHP could argue for precursors of HS for language in a common ancestor. To our knowledge, PT anatomical asymmetry was found only in great apes and has been shown to correlate in chimpanzees with manual preferences in relation to communicative gestures only, suggesting a left HS for gestural communication, as in human language. The aim of the present study is to investigate the neuroanatomical asymmetries of the PT in olive baboons (Papio anubis) as it may inform us about possible evolutionary continuity of this trait among primates. One hundred anatomical images were acquired in vivo at the Centre IRMf (Institut de Neurosciences de la Timone, Marseille) from anesthetized olive baboons housed in social groups at the Station de Primatologie CNRS. Surface areas of the PT were manually traced on a computer in both hemispheres for each subject (ANALYZE 11.0 software). In this communication, I will present two methods of delineation of the PT using anatomical MRI and descriptive statistics of PT surface area in 90 subjects, showing a significant leftward asymmetry. Such result might argue for the existence of a precursor of HS for language in the common ancestor of baboons, great apes and humans 30-40 MYA. Research was funded by the French 'Agence Nationale de le Recherche' (LangPrimate Project, P.I. Adrien Meguerditchian: ANR-12-PDOC-0014-01). This research complied with the European Directive 2010/63/EU. 


\title{
The Diversity of Locomotor Modes of Adapis (Primates, Eocene), as Reflected by Their Humeri
}

Judit Marigóa ${ }^{a}$ Nicole Verrière ${ }^{a}$, Marc Godinot $^{a, b}$

aUMR 7207 CR2P - C.N.R.S., M.N.H.N., U.P.M.C.-Paris 6, Département Histoire de la Terre, Muséum National d'Histoire Naturelle, and ${ }^{b}$ Ecole Pratique des Hautes Etudes, Paris, France E-Mail: judit.marigo@gmail.com

We studied humeral remains referable to Adapis species from old and new Quercy collections (late Eocene, France), which show a remarkable diversity in their morphological characters. At least five groups can be distinguished. A first group includes two specimens which show a rounded and laterally extended brachioradialis crest (one has a deltopectoral crest more extended distally than the other). A second group is distinct through an increased general robustness associated with a very proximal deltopectoral crest. It includes a specimen from the locality of Escamps, and a smaller one which might represent a closely related species. A third group includes the humerus described by Dagosto (1983) and one from the locality of Rosières. In the same locality, a fourth group represented by two distal extremities is found. These specimens have a lateral trochlear crest and a distinct zona conoidea (shallow groove on ROS2-79, deeper on Ros 640), whereas these traits are poorly developed in all the others. Furthermore, the posterior trochlea also differs markedly, being high in this fourth group, whereas it is laterally shallow in the previous one. These two groups must represent two closely related species differing only in their locomotor adaptation. Another humerus, ACQ 262, is also relatively similar to the latter two groups, however its proximal shaft is much broader, more robust, and it bears the best developed deltoid $\mathrm{V}$ of all specimens. Other differences among the humeri concern the articular head, somewhat flattened in many (restricted shoulder mobility), or more rounded and higher in lateral view; one has a greater tuberosity higher than the articular head. The size of the entepicondylar foramen varies, as well as the medio-distal extent of the medial epicondyle. The locomotor adaptations vary from highly quadrupedal forms to more agile climbers, some of them endowed with powerful grasping. These interpretations will be further discussed.

\section{Manual Processing of Plant Underground Storage Organs by Wild Bearded Capuchin Monkeys (Sapajus libidinosus)}

\author{
Luca Antonio Marino a, b Elisabetta Visalberghia , Patricia Izarc, Dorothy M. Fragaszy ${ }^{d}$, \\ Valentina Truppa ${ }^{a}$ \\ astituto di Scienze e Tecnologie della Cognizione, Consiglio Nazionale delle Ricerche (CNR), \\ and ${ }^{\mathrm{b}}$ Dipartimento di Scienze, Università degli Studi Roma Tre, Rome, Italy; ${ }^{\mathrm{C}}$ Department \\ of Experimental Psychology, University of São Paulo, São Paulo, Brazil; dDepartment of \\ Psychology, University of Georgia, Athens, Ga., USA \\ E-Mail: luca.marino90@libero.it
}

In dry climates some plants accumulate energy in underground storage organs (USOs) that are very rich in carbohydrates and minerals. Some argued that these organs had a crucial role as food resource for early hominins to colonize savannah habitats. USOs integrate the diet of modern humans, as well as non-human primates that have terrestrial habits. We studied a population of wild bearded capuchin monkeys (Sapajus libidinosus) living at Fazenda Boa Vista in Piauí, Brazil, which is an ecotone between cerrado and caatinga biomes. In this dry forest habitat, capuchins have terrestrial habits and forage on the ground to exploit a variety of foods including subterranean parts of plants, such as USOs. Capuchins' proficiency in extractive foraging is supposed to be improved by their high degree of manual dexterity, an ability that up to now has been

6th European Federation for Primatology Meeting
Folia Primatol 2015;86:235-386 DOI: $10.1159 / 000435825$ 
systematically investigated almost exclusively in captive settings. In this study, we aimed to assess manual skills employed by wild capuchins (i) to extract plant underground organs, (ii) to clean the soil from them, (iii) to remove the external part, and (iv) to tear apart the internal edible part before chewing and ingesting them. For three months, two observers opportunistically videotaped with high-resolution cameras 172 episodes of processing performed by 20 individuals of different sex-age classes. Videos were scored using frame-by-frame analysis and noting all actions requiring manual intervention. Overall, our findings indicated that processing did require strong coordination between hands and between hands and mouth, but not independent movement of digits. Grasping actions were mostly performed using power grip actions with food wrapped by the thumb in parallel with digits II-V. These findings indicate high degrees of manual dexterity in actions that require strength and coordination, rather than precision movements. We adhered to the IPS guidelines. Permission to work in Brazil was granted by IBAMA and CNPq.

\author{
Huntingtin Gene and Its CAG Repeats Number in Primates \\ Cristina Martínez-Labarga ${ }^{a}$, Chiara Zuccato ${ }^{b}$, Raffaele lennaco ${ }^{b}$, Giulio Formenti $^{b}$, \\ Cinzia Gellerac , Eva Martinez Nevado ${ }^{d}$, Gabriel Alcantara de la Fuente ${ }^{e}$, Agustin Lopez Goya ${ }^{f}$, \\ Maria Teresa Abellog, Pilar Dicerbo ${ }^{h}$, Valentina Truppa ${ }^{i}$, Klaus Friedrich $^{h}$, Elisabetta Visalberghi ${ }^{\prime}$, \\ Stefano Di Donato ${ }^{c}$, Olga Rickards ${ }^{a}$, Elena Cattaneo ${ }^{b}$ \\ aCenter of Molecular Anthropology for Ancient DNA Studies, Department of Biology, \\ University of Rome Tor Vergata, Rome, ${ }^{\text {b}}$ Department of BioSciences and Centre for \\ Stem Cell Research, Università degli Studi di Milano, and 'Department of Genetics of \\ Neurodegenerative and Metabolic Diseases, Fondazione IRCCS Istituto Neurologico Carlo \\ Besta, Milano, Italy; ${ }^{\mathrm{d} Z o o}$ Acquarium, ${ }^{\mathrm{e} F a u n i a, ~ a n d ~}{ }^{\mathrm{f}}$ Parques Reunidos, Madrid, and ${ }^{9}$ Parc \\ Zoologic de Barcelona, Barcelona, Spain; ' $F$ Fondazione Bioparco di Roma, and I'Istituto di \\ Scienze e Tecnologie della Cognizione, CNR, Rome, Italy \\ E-Mail: cristina.martinez@uniroma2.it
}

The Hdh gene arose with no CAG (cytosine-adenine-guanine) repeats in Dictyostelium discoideum around 800 million years ago, before the protostome-deuterostome divergence. The CAG then appeared in, and is unique to, the deuterostome branch. Two CAGs are found in Hdh in sea urchin (Strongylocentrotus purpuratus), the first species to carry a primitive nervous system, and two CAGs are present in amphioxus (Branchiostoma floridae), the first species to exhibit a rudimental hollow nerve tube and cephalization. Four CAGs are found in Hdh from the more evolved fishes, amphibians and birds. The CAG further expands in mammals and reaches its maximum length in humans. In Homo sapiens the Hdh gene is located in the short arm of chromosome 4 and is characterized by a CAG sequence repeated 9-35 times. Normal subjects have revealed an increase in grey matter with increasing length of the CAG repeat, indicating that CAG size can influence normal brain structure. However, CAG repeats exceeding 35 in number cause Huntington's disease, a neurodegenerative syndrome that occurs earlier due to a greater number of CAG repeats. Our hypothesis is that the progressive increase in CAG length in the Hdh gene observed during evolution may be implicated in the evolutionary changes that have occurred in developing adult nervous systems throughout vertebrate phylogeny, with a possible role for the CAG in newly emerging cognitive functions in the mammalian brain. We have collected genomic DNA and have begun to amplify the CAG tract from primates (Nycticebus pygmaeus, Eulemur fulvus albifrons, Lemur variegatus, Lemur catta, Sanguinus imperator, Cebus apella, Macaca fuscata, Macaca mulatta, Papio hamadryas, Chlorocebus aethiops, Cercocebus atys, Mandrillus sphinx, Colobus, Hylobates lar, Pongo, Gorilla gorilla, Pan troglodytes) in order to examine the inter- and intraspecific variability. Preliminary results will be shown. 


\title{
Food Manipulation and Hand Preference in Wild Western Gorillas
}

\author{
S. Masi ${ }^{a}$, E.J.M. Meulman ${ }^{a}, b$, A. San-Galli ${ }^{a}$, F. Aubaile ${ }^{a}$, S. Kriefa, A. Todd ${ }^{c}$, T. Breuerd $^{d}$, E. Pouydebat ${ }^{b}$
}

aMuséum national d'histoire naturelle, Département Hommes, Natures, Sociétés UMR 7206 Éco-anthropologie et Ethnobiologie, Musée de l'Homme, and ${ }^{\text {b} M u s e ́ u m ~ n a t i o n a l ~ d ' h i s t o i r e ~}$ naturelle, Département d'Ecologie et de Gestion de la Biodiversité UMR 7179 CNRS-MNHN, Mécanismes adaptatifs, des organismes aux communautés, Paris, France; 'Dzanga-Sangha Protected Areas, WWF, Bangui, Central African Republic; 'Wildlife Conservation Society Congo Program, Brazzaville, Republic of Congo

E-Mail: masi@mnhn.fr

In primates, food properties and the complexity of the task of processing affect manipulative techniques (e.g. grips, hand preferences). Even if roughly $90 \%$ of humans are right-handed, non-human primates show hand preference (right/left) depending on specific tasks, questioning the origins of human laterality. Hand preference is used as an indicator of brain lateralization for manual functions. After humans, gorillas possess a rather elongated thumb among great apes and are able to use precision grips and finer manipulation, involved in bimanual coordination. Our aim is to identify the extrinsic (body posture) and intrinsic features of the task (complexity e.g. unimanual task, bimanual extractive task) and object (e.g. size, position) affecting food manipulation strategies for different food types (e.g. stems, fruits) and manual preference in four groups of wild western gorillas at Bai-Hokou, Central African Republic, and Mondika, Republic of Congo $\left(\mathrm{N}_{\mathrm{obs} \text {. hours }}=1,555\right)$. Preliminary results indicate that food features (e.g. size, shape, extraction requirements) affect individual food-manipulation strategies (e.g. feeding techniques, number of fingers involved), as well as the requirement of various functional strategies (e.g. grip types, postural sequences, repositioning behaviour) during unimanual and different bimanual processing (e.g. different extractive foraging). Moreover, food size and position did not affect the hand preference of gorillas, being left-handed on unimanual tasks and right-handed on different bimanual tasks. We provide insights on human right-handedness, shedding light on the origin of lateralization within the Hominidae family. Furthermore, systematic microanalysis of manipulative actions could provide us with a powerful tool to improve our understanding of food-processing complexity across animal species and taxa. This research complied with the European Directive 2010/63/EU and the IPS Guidelines for the Use of Nonhuman Primates in Research.

\section{No Tail, No Transport! The Strategic Role of the Tail in Tool Carrying by Wild Capuchins (Sapajus libidinosus)}

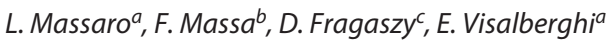

astituto di Scienze e Tecnologie della Cognizione, Consiglio Nazionale delle Ricerche,

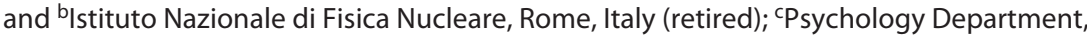
University of Georgia, Athens, Ga., USA

E-Mail: massaro.luciana@gmail.com

Carrying objects has been considered an important selective pressure favouring the evolution of bipedal locomotion in early hominins. Comparable behaviours by extant primates have been little studied, as few primates habitually carry objects bipedally. However, wild bearded capuchins (Sapajus libidinosus) living at Fazenda Boa Vista spontaneously and habitually transport stone tools by walking bipedally, allowing us to examine the characteristics of bipedal locomotion during objects transport by a generalized primate. We investigated kinematical and dynamical aspects of position and velocity of the centre of mass, trunk inclination, forelimb postures and the torques of the forces applied on each anatomical segment in wild bearded capuchin monkeys transporting objects, with particular attention to the tail and its role in balancing the body during transport. Our results indicate that body mass strongly affects the posture of transport and that 
capuchins are able to carry heavy loads bipedally with a bent-hip-bent-knee posture thanks to the 'strategic' use of their extendable tail; in fact, without this anatomical structure, constituting only $5 \%$ of their body mass, they would not be able to transport the loads they habitually carry. Our results suggest that the adoption of nearly or fully upright posture with extended hip and knee joints probably preceded habitual transport of heavy objects by human ancestors, who lacked a tail to offset the torque of an object held in front of the body.

\title{
All Species-Defining Characters Are Not Equal: Selection Regimes and Character Information in Nocturnal Strepsirhines
}

\author{
Judith Masters, Fabien Génin
}

African Primate Initiative for Ecology and Speciation (APIES), Department of Zoology and Entomology, University of Fort Hare, Alice, South Africa

E-Mail: jdthmasters@gmail.com

Formulations of the Phylogenetic Species Concept (PSC) - by far the most popular species concept among primatologists working with molecular datasets - are agnostic with respect to the evolutionary processes driving either the origin or maintenance of species. According to the PSC, any character that diagnoses a period of lineage independence can be used as the basis for recognition of distinct species. Advocates of this approach do not distinguish between geographic and reproductive isolation. Characteristics that form the basis of specific-mate recognition systems, however, are subject to well-characterised regimes of natural selection that differ at different stages of a species' lifetime. During speciation, strong directional selection will drive rapid coadaptation between signalling systems and transmission features of the environment, as well as between signal emitters and receivers. Once the new species is established, these same features of specific communication will incur strong stabilising selection, and become developmentally canalised. In contrast, traits facilitating the environmental adaptation of local populations (e.g. those related to predator avoidance or dietary adaptation) are likely to be subjected to fluctuating directional selection, and are unlikely to be canalised. These different regimes affect the variation and expression of morphological characters. In this presentation we discuss examples of reliable and unreliable diagnostic characters in cryptic, nocturnal strepsirrhine primates in the light of these selection scenarios, and argue in favour of diagnosing species in terms of aspects of the specific-mate recognition system, whenever possible.

\section{The Evolutionary Origins of Human Mind Viewed from the Study of Chimpanzees: The Parallel Efforts of Conservation and Welfare}

\section{Tetsuro Matsuzawa}

Primate Research Institute, Kyoto University, Kyoto, Japan

E-Mail: matsuzawa.tetsuro.8w@ kyoto-u.ac.jp

I compare cognitive development in humans with that of chimpanzees, both in the wild and in the laboratory. This talk aims to highlight the similarity and the difference of the two species and to introduce the efforts of conservation and welfare as well. Young chimpanzees possess exceptional working memory capacities, often superior to those of human adults. In contrast, their ability to learn the meaning of symbols is relatively poor. Human infants are typically raised by more than one adult, not only the mother, but also the father, siblings, grandparents and the other members of the community. The human infant is characterized by the stable supine posture of the neonate that enables face-to-face communication via facial expressions, vocal exchange, 
manual gestures and object manipulation because both hands are free. The stable supine posture makes us human. The socio-cognitive development in humans may be integrally linked to this mother-infant relationship and the species-specific way of rearing the children. In the process of studying chimpanzees, my colleagues and I have promoted conservation in wild chimpanzees by the 'Green Corridor' project of planting trees to connect the isolated habitats of Bossou and Nimba, Guinea. We have also promoted environmental enrichment of captive chimpanzees and succeeded in completely stopping invasive biomedical research on chimpanzees.

\section{Using Spatially Explicit Capture-Recapture Techniques to Survey a Population of Yellow-Cheeked Crested Gibbons in the Seima Protection Forest, Cambodia}

Sharon McCabe, Susan M. Cheyne

Oxford Brookes University, Oxford, UK

E-Mail: sharoncjmccabe@gmail.com

Spatially explicit capture-recapture (SECR) modelling is emerging as an innovative new way to estimate population densities; having been effectively used for estimating mammalian densities from fixed camera traps for many years. By integrating a state model (to describe the distribution of home ranges in the study area) with an observation model (the probability of detecting individuals at a particular detector within the study area), SECR analysis presents the opportunity for a well-rounded density estimate of a population within a fixed area. As this technique has rarely been used on primates, gibbons represent an ideal candidate due to their distinct, structurally complex calls which may be used as capture-recapture data. Surveys of these arboreal, cryptic primates often produce underestimates as individuals are able to flee quickly and unnoticed by researchers performing surveys by line transects or plot sampling. In order to address this issue, SECR analysis was performed on a population of yellow-cheeked crested gibbons (Nomascus gabriellae) in the Seima Protection Forest, southeast Cambodia. During data collection, groups of three listening posts each, set along 14 independently placed transects within the group's home range were used to record calling data. The points of detection for each call were then used to triangulate the position and number of gibbons within each listening area. These data were treated as capture-recapture data and were then run through SECR analysis in R in order to produce a population density estimate. The results of this survey were compared to annually-taken line transect survey data from the same population in order to examine the effectiveness of the technique on gibbons. SECR analysis presents a less-invasive and less labour-intensive survey technique for gibbons and implementation across study sites with multiple species of gibbons would be highly beneficial to conservation efforts worldwide.

\section{Survival in the Fragments: Chimpanzees (Pan troglodytes schweinfurthii) Exploit New, Non-Native Cultivars for Nesting and Feeding in a Human-Dominated Landscape, Western Uganda}

Maureen McCarthy ${ }^{a, b}$, Jack Lester ${ }^{b}$, Craig Stanford ${ }^{a}$

aUniversity of Southern California, Los Angeles, Calif., USA; bMax Planck Institute for

Evolutionary Anthropology, Leipzig, Germany

E-Mail: msmccart@usc.edu

As habitat loss and fragmentation place growing pressure on endangered non-human primates, researchers find increasing evidence of novel responses in their behaviour. In western Uganda, chimpanzees in Hoima and Masindi Districts inhabit a mosaic landscape comprised of 
forest fragments, human settlements and agricultural land. We non-invasively recorded nests and feeding evidence of unhabituated chimpanzees in this region over a 15-month period. We found extensive evidence of nesting in cultivated tree species, including eucalyptus (Eucalyptus grandis), guava (Psidium guajava), cocoa (Theobroma cacao) and pine (Pinus caribea). Additionally, we found frequent instances of nesting at low heights or on the ground, which may result from a lack of suitable nesting trees or from an attempt by chimpanzees to avoid human detection. Finally, as with chimpanzees elsewhere in this region, we found broad evidence of cultivar feeding. However, this included the consumption of novel cultivar species not reported to be consumed elsewhere. Such evidence suggests chimpanzees respond flexibly to mitigate anthropogenic pressures in human-dominated landscapes. The limits of such flexibility, however, remain unknown. Further research is needed to examine systematically the factors influencing the use of such resources and to understand better the extent to which chimpanzees can persist while relying upon them. This work complied with International Primatological Society Guidelines for the Use of Nonhuman Primates in Research.

\section{Chimpanzee Foraging Responses to Fast-Changing Environments with Evidence for Intercommunity Variation}

\section{Matthew McLennan}

Anthropology Centre for Conservation, Environment and Development, Oxford Brookes University, Oxford, UK

E-Mail:mmclennan@brookes.ac.uk

The ability of wild animals to respond flexibly to anthropogenic environmental changes, including agriculture, is critical to their survival in human-impacted habitats. Recent research has revealed that chimpanzees (Pan troglodytes) consume agricultural crops throughout tropical Africa, with crop selection reflecting a species-typical preference for fruit. Detailed data on chimpanzee crop-feeding are available from 2 sites: Bossou (Guinea), with long-term exposure to agriculture, and Bulindi (Uganda), with more recent exposure. Both communities exploit a range of crops, with more accessible crops eaten most frequently at both sites. Crop selection at Bossou is more omnivorous (less fruit-biased) than at Bulindi, where chimpanzees ignore most non-fruit crops, suggesting crop selection by chimpanzees diversifies over time (i.e. becomes less-selective). Chimpanzees at Bulindi inhabit small forest fragments on agricultural land. Between 2007 and 2014 forest fragments were reduced in size by $>80 \%$ as farmers converted forest into farmland. Long-term analysis of the chimpanzees' diet reveals how they are responding to these rapid habitat changes. The chimpanzees incorporate human crops into their seasonal foraging strategy: when wild fruit is scarce they increase consumption of energy-rich crops whilst also eating more non-fruit plant fibre (leaves and pith). However, comparing feeding data in 2007 and 2012-14 (after major forest clearance) shows that the chimpanzees now eat less fruit overall and more lower-quality fibrous piths and leaves, and a greater proportion of fruits eaten are agricultural crops. The data also show their use of individual crops is changing, related to fluctuating patterns of resource availability. Understanding dynamic responses of endangered wildlife to agriculture exposure can help predict their current and future adaptability to fast-changing landscapes and inform conservation strategies. 


\title{
Motor Planning for Grasping in Different Contexts by Tufted Capuchin Monkeys (Sapajus spp.)
}

\author{
Giusy Meglio $^{a, b}$, Valentina Truppa ${ }^{a}$, Gloria Sabbatini ${ }^{a}$
}

aUnità di Primatologia Cognitiva, Istituto di Scienze e Tecnologie della Cognizione del CNR, and 'bapienza Università di Roma, Rome, Italy

E-Mail: giusy.meglio87@gmail.com

Studies on motor planning and action selection in object use reveal that what we choose to do in the present moment differs depending on what we intend to do next. In particular, many studies have shown that human adults adopt initially uncomfortable hand postures to accommodate later task demands (i.e., the end-state comfort effect). Recent studies on action planning in non-human primates belonging to different taxonomic groups provided contrasting results. Here we tested whether capuchin monkeys (Sapajus spp.), natural tool users, would show the end-state comfort effect in two different contexts. In Experiment 1, ten capuchin monkeys had to grasp a horizontal dowel with either the left- or right-end baited. If action selection is influenced by the intended subsequent movement (comfortably bringing the baited end of the handle to the mouth), then capuchins would prefer the grip that places the thumb-side of the hand towards the baited end; i.e. they would prefer a radial grip. In Experiment 2, seven capuchins needed to use a dowel that rested on a bracket that could be horizontally rotated (thus altering how the subject could grasp it) to dislodge an out-of-reach food reward. Also in this case, a radial grip allows the possibility to complete the task in a more comfortable way. In both experiments, capuchin monkeys adopted a radial grip significantly more often than other types of grips to accommodate subsequent task requirements, thus demonstrating the end-state comfort effect. Cognitive and motor skills potentially affecting action planning in primate species are discussed. Our research protocols complied with the European Directive 2010/63/EU and were approved by the Italian Health Ministry (License No. 132/2014-C).

\section{Brain, Gestures and Mouth in Baboons (Papio anubis): What May They Tell Us about the Origins of Some Language Properties?}

\author{
Adrien Meguerditchian ${ }^{a, b}$, Marie Bourjade ${ }^{c}$, Damien Marie ${ }^{a, b}$, Scott Love ${ }^{a}$, Pauline Fresnais ${ }^{a, b}$, \\ Marie Plouvier ${ }^{a, b}$, Konstantina Margiotoudi ${ }^{a}, b, e$, Muriel Roth $^{d}$, Romain Lacoste ${ }^{b}$, \\ Bruno Nazarian ${ }^{d}$, Alice Bertello ${ }^{a, b}$, , Florence Gaunet ${ }^{a}$, Jean-Luc Anton ${ }^{d}$, Olivier Coulon ${ }^{g}$ \\ aLaboratoire de Psychologie Cognitive UMR7290, Brain and Language Research Institute, \\ Université Aix-Marseille/CNRS, Marseille, 'b Station de Primatologie CNRS UPS846, Rousset, \\ 'Octogone, EA4156, Jean Jaurès University, Toulouse, and d Centre IRMf, Institut des \\ Neurosciences de la Timone UMR7289, Aix-Marseille Univ./CNRS, Marseille, France; \\ eGroningen University, Groningen, The Netherlands; ${ }^{\mathrm{f} E c o l e ~ N a t i o n a l e ~ V e ́ t e ́ r i n a i r e, ~ T o u l o u s e, ~}$ \\ and SLaboratoire des Sciences de I'Information et des Systèmes UMR 6168, Aix-Marseille \\ Univ./CNRS, Marseille, France \\ E-Mail: adrien.meguerditchian@univ-amu.fr
}

Within a comparative approach to the origin of language, investigations of the communication gestural system in great apes have been well studied. Such a comparative framework extended to primate species other than great apes is needed in order to help reconstruct the phylogeny of some language properties, such as intentionality, flexibility of learning, hand-mouth motor links and left hemispheric lateralization. In the present paper, I will review our published and ongoing work from the 3-years-old 'LangPrimate' project conducted on the features of communicative gestures in captive olive baboons Papio anubis living in social group at the Station de

6th European Federation for Primatology Meeting
Folia Primatol 2015;86:235-386 DOI: $10.1159 / 000435825$ 
Primatologie CNRS (Rousset, France). This project includes the collection of behavioural data on gesture and on the motor system, as well as the analysis of neuroanatomical MRI data from our recent collection of 100 cerebral images acquired in vivo at the Centre IRMf (INT, Marseille). I will briefly report (1) typical criteria of intentional communication (gaze alternation, adjustment to the recipient's attentional state, signal persistence) in the ritualized food begging gestures directed at humans, (2) emergence of novel intentional gestures in the baboons' repertoire (such as clapping, cage banging, referential food presenting) indicating the flexibility of the gestural system, (3) evidence of strong motor links between the hand and mouth in the manual fine activities such as grooming which elicited differential lips movements, (4) specific patterns of gestural lateralization, as well as language-like hemispheric structural asymmetries for the planum temporale in the baboons brain. These findings will be discussed within a comparative framework in primates about the evolution of communicative systems. Research was funded by the French 'Agence Nationale de le Recherche': ANR-12-PDOC-0014-01 (LangPrimate Project, P.I. Adrien Meguerditchian) and complied with the European Directive 2010/63/EU.

\section{Audience Effect in Wild Vervet Monkeys (Chlorocebus aethiops) \\ Stéphanie Mercier ${ }^{a, c}$, Erica van de Waal ${ }^{b, c}$, Klaus Zuberbühler ${ }^{a, c}$ \\ aDepartment of Comparative Cognition, University of Neuchâtel, Neuchâtel, and \\ ${ }^{\mathrm{b}}$ Anthropological Institute and Museum, University of Zurich, Zurich, Switzerland; Inkawu Vervet Project, Mawana Game Reserve, Swart Mfolozi, South Africa \\ E-Mail: stephanie.mercier@unine.ch}

Although vervet monkeys warn their conspecifics about external events by producing distinct vocalizations, little is known about how their vocal behaviour is affected by the presence of an audience. Fake predator, playback and food experiments are used to investigate this in four contexts: encountering predators, discovering food, recruiting help and greeting another individual. While it is well known that alarm call production is influenced by the composition of the audience, few studies have been realized on how vervet monkeys can modify the acoustic structure of their call according to the presence of other individuals while discovering food. Moreover, significant acoustical differences were found between screams produced by victims or aggressors. As a consequence, variation of screams produced by vervets' opponents might help bystanders to give support only when necessary. Lastly, the call production of vervet monkeys while encountering another individual seems to be influenced by the presence of an audience since they might use these greeting calls to show the acceptance of their social rank. Getting a better understanding of the communication system of the vervet monkeys and comparing it with other primates such as baboons, mangabeys and chimpanzees might shed light on how the complexity of the communication system evolved through the primate lineage, and thus help us understand part of the evolution of human language. 


\title{
What Do Rhesus Macaques Know about Conspecifics' Dominance Ranks?
}

\author{
H. Meunier ${ }^{a, b}$, L. Pagéa, J.-J. Roeder ${ }^{a, c}$
}

${ }^{a}$ Centre de Primatologie de I'Université de Strasbourg, Niederhausbergen, ' $L a b o r a t o i r e$ de Neurosciences Cognitives et Adaptatives, UMR 7364, Université de Strasbourg, and 'Centre National de la Recherche Scientifique and Université de Strasbourg, UMR7178, Institut Pluridisciplinaire Hubert Curien, Département Ecologie, Physiologie et Ethologie, Strasbourg, France

E-Mail: hmeunier@unistra.fr

Social animals, including primates, must behave appropriately in various social situations to prevent conflicts with their conspecifics by optimizing their social interactions and so their social relationships. Dominance is one of the social factors that most influences the daily interactions between group members in primates' societies. This, it appears crucial for each individual to know its place in the dominance hierarchy and act accordingly. We already know that monkeys can extract dominance information through observation of social interactions between unfamiliar conspecifics. However little is known of the nature and precision of their knowledge about the dominance ranks of their own group members, mostly because previous research was based on indirect observation - versus 'asking' the subject himself. In the present study, we proposed a training protocol consisting of four steps of increasing complexity, in which the subjects had to consistently select the dominant individual when presented with pairs of photographs of their group mates. This training allowed us to test rhesus macaques (Macaca mulatta) directly and individually on their own social knowledge. We worked on a group of nine macaques among which two individuals succeeded in the four steps of our training and were then tested with all possible pairs of group members that had not been used during the training phases, i.e. eleven distinct pairs. Both individuals significantly chose the dominant versus the subordinate in pairs of group mates. This study is the first to provide clear evidence that macaques know the precise dominance order of the group to which they belong and are aware of other's dominance relationships. Moreover, this protocol can now be used for testing larger groups and investigating factors that could influence their knowledge of such topics as kinship, sex, age, friendship, the group's social structure or the subject's own social status.

\section{First Field Surveys and Red List Assessment of Two Bornean Slow Lorises (Nycticebus menagensis and N. kayan) Using Local Knowledge in Sabah, Borneo}

Priscillia Miard ${ }^{a, b}$, K.A.I. Nekaris ${ }^{a, b}$

${ }^{a}$ Nocturnal Primate Research Group, Oxford Brookes University, Oxford, UK; 'bittle Fireface Project, Cisurupan, Garut, Java, Indonesia

E-Mail: priscillia.miard-2014@brookes.ac.uk

Slow lorises (Nycticebus spp.) are small, cryptic nocturnal primates found throughout SE Asia that are threatened by habitat loss and the illegal wildlife trade. Over the last decade, revisions in taxonomy mean that five of the eight species are listed by the IUCN as Vulnerable or Critically Endangered, with three new taxa in Borneo currently Data Deficient. In this research project, we aimed to provide the first systematic field data from Sabah (Malaysia) focusing on two species of lorises: Nycticebus menagensis (VU) and N. kayan (DD). Our preliminary work in the area has already shown that these species occur in both protected forests and in anthropogenically disturbed habitats. In order to gain knowledge on the range and potential overlap of these taxa, as well as their use by humans, we focused on the local knowledge, conducting a series of

6th European Federation for Primatology Meeting
Folia Primatol 2015;86:235-386 DOI: $10.1159 / 000435825$ 
interviews with local people in remote areas as well as with staff running tourist hotels in forested areas. Abundance estimates of species have been made through forest surveys via occupancy modelling, and observations with the aid of an infrared video camera. Examinations of locally caught pet lorises have also been undertaken. Hopefully, our data provide a basis for a conservation assessment for the new species $N$. kayan as well as gain an understanding about the populations of remaining lorises in these regions of Borneo. The data presented in this poster are the result of a three-month field study with 172 interviews and 16 plots transect at night to understand how the local beliefs affect slow loris conservation and their abundance. Results show that ancient taboos about slow lorises are decreasing because of an expanding globalization, and that this issue needs to be addressed with more intensive education projects in the field. Our survey using occupancy modelling allows for a clear idea of the range of the two different species but further investigation is needed to estimate population densities.

\title{
Does Self-Control Rely on a Limited Resource in Tufted Capuchin Monkeys (Sapajus spp.)?
}

\author{
Antonia Micucci ${ }^{a}$, Emanuele Gori ${ }^{a}$, Francesca De Petrillo ${ }^{a, b}$, Valentina Truppa ${ }^{a}$, Dan Arielyc, \\ Elsa Addessia \\ aUnità di Primatologia Cognitiva, Istituto di Scienze e Tecnologie della Cognizione, CNR, \\ and ${ }^{\mathrm{b}}$ Dipartimento di Biologia Ambientale, Sapienza Università di Roma, Rome, Italy; \\ 'Duke University, Durham, N.C., USA \\ E-Mail: anto189@hotmail.it
}

Since failure of self-control has enormous personal consequences, several models attempted to explain why self-control breaks down. According to the Strength Model, self-control is a limited resource. Previous acts of self-control or taking part in highly demanding cognitive tasks may deplete this resource causing short-term impairments in subsequent acts of self-control. Studies on humans and dogs found that exerting self-control reduces blood glucose levels and that, if self-control is depleted, glucose ingestion can restore this capacity. However, a recent meta-analysis questioned the robustness of the depletion effect in humans. Here we investigated the validity of the Strength Model in capuchin monkeys (Sapajus spp.). We tested five capuchins in the Accumulation task, in which food items were accumulated within reach of the subject for as long as the subject refrained from taking them. We evaluated how exerting self-control in each trial of the Accumulation task affected this capacity over subsequent trials (within-task depletion) and whether capuchins' performance in the Accumulation task decreases when tested before receiving their daily meal rather than after consuming it (Energy Depletion Experiment) and after being tested in a cognitively demanding task rather than in a less cognitively demanding task (Cognitive Depletion Experiment). In both experiments, capuchins' performance in the Accumulation task decreased over trials within each session. Hence, as predicted by the Strength Model, capuchins seem to be vulnerable to within-task depletion effects. In contrast, we did not find a significant effect of both cognitive and energy depletion. These results failed to support some previous outcomes obtained in humans and dogs, but they add to the growing body of studies that did not find a depletion effect in humans. This study complied with the Directive 2010/63/EU and was approved by the Italian Health Ministry (DM 12/2011-C and DM 123/214-C). 


\author{
Play in Wild Juvenile Chimpanzees (Pan troglodytes) \\ Calogero Montedoro ${ }^{a, b}$, Hans Van Dyck ${ }^{a}$, Zarin P. Machanda ${ }^{b}$, Richard Wrangham ${ }^{b}$ \\ aUniversité catholique de Louvain, Earth and Life Institute, Louvain-la-Neuve, Belgium; \\ ${ }^{b}$ Harvard University, Cambridge, Mass., USA \\ E-Mail: calogero.montedoro@uclouvain.be
}

The emergence of sociality in the animal kingdom is a major step in evolution. Social life is characterized by daily and repeated interactions with conspecifics. Throughout evolution, diversification and complexity of social interactions is observed. In particular, many primates live in social groups. They coordinate their activities by a variety of social interactions and communication forming strong social bonds. These complex interactions are often expressed as dominance, cooperation, coalition and grooming. In order to integrate the rules of social life, play behaviours are considered to be a particularly effective and universal learning engine, which includes knowledge of congeners. In addition, play contributes to social cohesion and development of cognitive skills needed for life in society. The aim of this research project is to test these assumptions about sociobiology of primates' play behaviour, particularly in juvenile chimpanzees (Pan troglodytes). In our study, we focus on a community of wild chimpanzees resident in the Kibale Forest National Park, Uganda. In accordance with the ontogeny of play behaviour, we observed that the type of play sessions differed depending on the age of the individual and his/her partner. Moreover, Baldwin \& Baldwin's 'hypothesis of social cohesion' predicts that play can help establish useful social relations for the future. Therefore, we can expect that, because chimpanzees show a male philopatry, the social play profits are lower for females than for males (who themselves will remain in the group). Social play activities were found to be more frequent in males than in females, who spend more time in solitary and mothering games; this corroborates Groos \& Baldwin's 'practice hypothesis'. Finally, we observed the emergence of preferences in the choice of specific playing partners (sex, age, kinship). This research complied with the European Directive 2010/63/EU and the International Primatological Society (IPS) Guidelines for the Use of Nonhuman Primates in Research.

\author{
Are Gibbons Really Left-Handed? \\ L. Morino $^{a}$, M. Uchikoshi ${ }^{b}$, F. Bercovitch ${ }^{b}$, W.D. Hopkins ${ }^{c}$, T. Matsuzawa $^{b}$ \\ ${ }^{a}$ CNRS, Paris, France; ${ }^{b}$ Kyoto University, Kyoto, Japan; ${ }^{c}$ Yerkes Regional Primate Research \\ Center, Atlanta, Ga., USA \\ E-Mail: Imorino@gmail.com
}

Many of the links between brain lateralization and vocal and gestural communication are still hotly debated. Hylobatids have been rather neglected in this research area, yet they can provide quite important insights: (1) they are one of humans' closest relatives sharing a complex vocal repertoire, hence they might share similar neurological pathways for complex vocalization; (2) their adaptation to arboreality has produced unique postural constraints; (3) the little that is known about laterality in gibbons is contradictory (captive studies provided conflicting results, while a field study on siamangs reported a population-level left-hand preference). To clarify matters, we investigated hand preference in captive siamangs $(n=22)$ and gibbons ( 4 species, $n=21)$ in Japanese facilities. We had a large sample size, controlled for possible confounding factors (posture, enclosure limitations, motivation) and used well-established testing protocols (unimanual task: simple reaching; bimanual task: tube test). I discuss the results in light of previous findings and suggest further steps to correlate lateralization in motor tasks with singing - the complex communication of gibbons. 


\title{
Welcome Back: Behavioural and Physiological Responses of Female Bonobos (Pan paniscus) to Fusions
}

\author{
Liza R. Moscovice, Tobias Deschner, Gottfried Hohmann \\ Max Planck Institute for Evolutionary Anthropology, Leipzig, Germany \\ E-Mail: Liza_moscovice@eva.mpg.de
}

In species with a high degree of fission-fusion social dynamics, fusions provide opportunities for individuals to reinforce or strengthen social relationships, but they may also trigger social conflict. We used a combination of captive and field studies to evaluate post-fusion social dynamics among female bonobos (Pan paniscus), who have been characterized by high levels of gregariousness and cooperation among non-kin. Captive studies occurred at Frankfurt Zoo, in a facility designed to simulate naturalistic fission-fusion social dynamics. Field studies occurred at Luikotale field site, DRC. We tested whether fusions led to behavioural or physiological indicators of social tension, whether females have mechanisms to reduce or avoid post-fusion social tension and whether a female's choice of association and affiliation partners post-fusion reflects differences in the quality of her intra-sexual relationships. Aggression increased following simulated fusions in captivity, but consisted only of low-intensity displays or charges and there was no effect of fusions on urinary cortisol levels. Post-fusion socio-sexual solicitations and sociosexual interactions increased between reunited females, and females who had been separated for longer exchanged more socio-sexual solicitations. We compare these results with patterns of post-fusion social behaviour among wild female bonobos, and conclude that socio-sexual interactions play a role in reintegrating female bonobos into social groups following fusions. All research was approved by the Ethics Committee of the Max Planck Gesellschaft and was conducted in accordance with the IPS Guidelines for the Use of Nonhuman Primates in Research.

\author{
Nest Site Selection in the Chimpanzees of Rubondo Island, Tanzania \\ Nadezda Josephine Msindai \\ University College London, London, UK \\ E-Mail: n.msindai.09@ucl.ac.uk
}

Each day, post-weaning great apes build platforms ('nests') in trees or occasionally on the ground to sleep in at night. Functional hypotheses suggest comfort, thermoregulation, predator or pathogen avoidance as primary reasons for why chimpanzees construct nests. I studied nest building in chimpanzees (Pan troglodytes) belonging to a population introduced to Rubondo Island National Park, in Tanzania, almost 50 years ago. Over 21 months from 2012 to 2014, I collected information on 1,170 individual nests not older than one month. The influence of predation risk on nest building can be excluded, because Rubondo Island does not contain large predators such as leopards that could pose a threat to the apes. Predictions for the thermoregulation hypotheses were not met. Although, it is predicted that on cold days great apes should build nests higher up in trees in order to benefit from early sun. Similarly rainfall did not influence the height of nests. Though, it is expected that chimpanzees should select to build nests higher up in trees with no vegetation cover overhead during the rainy season in order to reduce disturbance from dripping water above once rain has stopped. I should point out that unlike other chimpanzee habitats, climatic seasonality is less pronounced on Rubondo Island. Nevertheless, Rubondo chimpanzees showed a high selectivity for a handful of tree species. Thus $82 \%$ of nests were built in three tree species: Synsepalum brevipes, Pancovia turbinata and Drypetes gerrardii, despite the fact that they represent only $25 \%$ of specimens on random phenology transects. Various factors are likely to influence nest site selection in chimpanzees. I am currently exploring if these trees are associated with potential medicinal properties, for example repelling insects that transmit diseases such as malaria. Research was approved by the Tanzanian Wildlife Research Institute and complied with European directive 2010/63/EU.

326
Folia Primatol 2015;86:235-386 DOI: $10.1159 / 000435825$ 6th European Federation for Primatology Meeting 


\title{
The Chimpanzees of Rubondo Island: Genetic Data Reveal Their Origin
}

\author{
Nadezda Josephine Msindai ${ }^{a}$, Volker Sommer ${ }^{a}$, Christian Roos $^{b}$
}

aDepartment of Anthropology, UCL, London, UK; ' PPrimate Genetics Laboratory, German

Primate Centre, Leibniz Institute for Primate Research, Göttingen, Germany

E-Mail: n.msindai.09@ucl.ac.uk

The subspecific classification of chimpanzees (Pan troglodytes) currently comprises the taxa verus, ellioti, troglodytes and schweinfurthii. All are classified as endangered on the IUCN Red List of Threatened Species. However, the geographic origin of many chimpanzees held in sanctuaries and other institutions is often not known. This is also the case for the descendants of $17 \mathrm{chim}$ panzees that were introduced, between 1966 and 1969, by the Frankfurt Zoological Society into what later became Rubondo Island National Park. The apes had been caught in the wild and kept in captivity in European zoos for various periods of time prior to their release. One individual originated from Sierra Leone and another possibly from Guinea. The provenance of the remaining 15 chimpanzees was unknown, although it was assumed that all came from West Africa. Between 2012 and 2014 we collected chimpanzee faeces $(n=214)$, food wadges $(n=2)$ and hairs $(\mathrm{n}=51)$ from underneath and within night nests and from the forest floor. We successfully sequenced the hypervariable D-loop region of mtDNA from more than 200 samples. The data revealed that the founder population was composed of two sub-species. These indeed include the West African taxon verus, but also the Central African taxon troglodytes. Such information provides baseline data for the management of this unique chimpanzee population.

\section{Do Orangutans Like to Paint? Preliminary Study on Stress Variations in Captivity by Salivary Cortisol Levels and Behavioural Observations}

Victor Narat ${ }^{a}$, Baptiste Sadoughib, Morgane Debuigne ${ }^{c}$, Michel Saint-Jalme ${ }^{a}$, Sabrina Kriefa

aMuséum national d'Histoire naturelle, Ecoanthropologie et ethnobiologie, UMR 7206,

Paris, ${ }^{b}$ ONIRIS, Nantes, and ${ }^{c}$ Vet Agro Sup, Lyon, France

E-Mail: victor.narat@gmail.com

Boredom and stress in captive primates can lead to stereotypic and self-centred behaviours. Chronic stress, with a rise of glucocorticoid concentration, is a threat for primate health because of immune system depression. Environmental enrichment is used to decrease the stress in captive primates. This preliminary study aims to evaluate the effect of painting material as a potential enrichment for orangutans. Salivary cortisol levels and behavioural observations were used to evaluate stress levels. Experiments were carried out on three females orangutans housed together (45, 26 and 9 years old) in July and August 2014 in Paris, France. Painting material was composed of four colours (yellow, green, blue, red) of paint for children (non-toxic) and three sheets of drawing paper. Saliva was sampled using a rope impregnated with honey at 8:30 a.m. and then at the end of the 30 min painting session (from 9:45 a.m. to 10:15 a.m.). In order to evaluate circadian variations of salivary cortisol, five salivary samples per day (every two hours between 8:30 a.m. and 4:30 p.m.) per individuals were collected during four days of the painting sessions. The salivary cortisol dosage was performed by Enzyme-linked immunosorbent assay method (Salimetrics $\mathrm{Kit}^{\circledR}$ ). In addition, $30 \mathrm{~min}$ focal individual sampling was used during and outside the painting sessions and to evaluate the levels of stereotypic and self-centred behaviours expressed. Each individual participated in at least one to seven painting sessions. Whereas there was no significant variation in salivary cortisol over the day, there was a decrease of salivary cortisol level after the painting session ( $\mathrm{n}=10$ pairs). Stereotypic and self-centred behaviours were also reduced during the painting sessions. These two results indicate a stress reduction during painting sessions. These preliminary results are discussed for great ape management in captivity and welfare improvement. 


\title{
Gender Differences in Marking Behaviour in Sapajus flavius (Schreber, 1774) Inhabiting a Fragment of Atlantic Forest in Northeastern Brazil
}

\author{
Eudécio Carvalho Neco a , Mônica Mafra Valença-Montenegro ${ }^{b}$, Carla Soraia Soares de Castroc, \\ Luiz Carlos Serramo Lopez ${ }^{a}$ \\ aUniversidade Federal da Paraíba/Departamento de Ecologia e Sistemática/Programa de \\ Pós Graduação em Ciências Biológicas, and ${ }^{\mathrm{b} C e n t r o ~ N a c i o n a l ~ d e ~ P e s q u i s a ~ e ~ C o n s e r v a c ̧ a ̃ o ~}$ \\ de Primatas Brasileiros (CPB)/ICMBio, João Pessoa, and 'Universidade Federal da Paraíba/ \\ Departamento de Engenharia e Meio Ambiente/Programa de Pós Graduação em Ecologia e \\ Monitoramento Ambiental, Rio Tinto, Brazil \\ E-Mail: csscastro9@gmail.com
}

Sapajus flavius (Schreber, 1774) is a Neotropical primate species found in the Atlantic Forest in northeast Brazil. Rediscovered in 2006, this taxon is classified as threatened in the IUCN Red List. However, little is known about the social behaviour of this species, particularly the role of the dewlap, an epidermal structure connected loosely around the neck that is present only in adult males. The aim of this study was to determine if the dewlap is a structure used in marking behaviour. From January 2014 to January 2015, a group of approximately 60 blond capuchin monkeys inhabiting a fragment of Atlantic Forest in the state of Paraiba (Brazil) was observed using the 'all occurrences' method. The body parts used by the individuals in marking behaviour and the substrates where the markings occurred were also recorded. There was a total of $375 \mathrm{~h} 49$ min of sampling effort and $76 \mathrm{~h} 46$ min of observations. Fourteen behaviour categories were recorded, with a total of 491 records. Marking represented $6 \%$ of total behaviour records. The dewlap was used by adult males to mark different substrates: tree trunk (62\%), feeding box (17\%), food (14\%), termite nests (3\%) and other individuals (3\%). There was a significant difference in the frequency of marking by adult males compared to females and non-adults (chi-square, Yates corrected, $\chi^{2}=19.950$, d.f. $=1, \mathrm{p}<0.001$ ). The results suggest that the dewlap is a structure associated with marking behaviour in $S$. flavius adult males.

\section{Venom in Furs: Pelage Variation and Its Implications for Slow Loris Evolution}

\author{
K.A.I. Nekaris ${ }^{a}$, Nabajit Das ${ }^{a, b}$ \\ aNocturnal Primate Research Group, Oxford Brookes University, Oxford, UK; \\ bPrimate Research Centre NE India, Assam, India \\ E-Mail: anekaris@brookes.ac.uk
}

Contrasting facial masks and dark dorsal stripes in mammals have been argued to play a role in species or individual recognition, or as aposematic signals serving as warnings against predators. Pelage variation has also been used to describe new species, such as has been the case for Asia's slow lorises (Nycticebus spp.). Here we consider the role of pelage variation based on two long-term field studies of the Bengal slow loris in NE India (N. bengalensis - 2008-2010) and the Javan slow loris in West Java (N. javanicus - 2012-2015). To examine species and individual recognition, we compared the facial masks of $12 \mathrm{~N}$. bengalensis and $35 \mathrm{~N}$. javanicus for nine recommended species-specific characters. Individuals varied in colour and in three characters of circumocular patch shape, suggesting a role for individual recognition. Five of the nine characters consistently assigned the animals to species. Both species retained their dark dorsal stripes throughout the year, both consumed more than 50\% exudates as part of their diet and both slept in bamboo or branch tangles. Living in more seasonal forest, $N$. bengalensis changed the colour of its coat during the cool season when leaves were scarce. We compared loris colour to the colour 
of common gum trees and show evidence for background matching and use of the dorsal fur as an aposematic signal. Finally, both species give birth to infants that are black and white in colour. Specimens of such individuals in museums have been ascribed to different taxa, and we warn against using colour alone to describe new loris taxa. The slow loris is one of the world's only venomous mammals. We suggest that black and white coats in infants also serve as a warning signal to potential predators. We also consider the role of the striking facial mask and dorsal stripe as a potential example of Müllerian mimicry of cobras. All research followed the International Primatological Society (IPS) Guidelines for the Use of Nonhuman Primates in Research.

\author{
Diversity of Hand Grips and Laterality in Wild African Apes \\ Johanna Neufuss ${ }^{a}$, Tatyana Humle ${ }^{a}$, Tobias Deschner ${ }^{b}$, Martha M. Robbins $^{b}$, Giulia Siriannib, \\ Christophe Boesch ${ }^{b}$, Tracy L. Kivella, \\ aschool of Anthropology and Conservation, University of Kent, Canterbury, UK; \\ Departments of brimatology and CHuman Evolution, Max Planck Institute for Evolutionary \\ Anthropology, Leipzig, Germany \\ E-Mail:.jn259@kent.ac.uk
}

Comparative studies of primate grasping and manipulative behaviours in captivity have highlighted, among others, two human abilities that are generally considered unique compared with other primates: (1) the use of forceful precision and power squeeze grips involving the use of the thumb, and (2) a species-wide dominant use of one hand (usually the right hand), known as laterality. However, recent research has highlighted a diversity of precision and power grips in general among many non-human primates, and there is much debate around the potential for population-level or species-wide laterality in non-human primates. The majority of this research to date has been done on captive primates performing specific manipulative tasks, which may bias or confound these species comparisons. Comparatively little research has been done on hand use in wild primates, especially during natural, non-manipulative activities, including locomotion. Here, we investigate hand use during locomotor and non-locomotor behaviours in wild mountain gorillas (Gorilla beringei beringei, Bwindi Impenetrable National Park, Uganda), wild chimpanzees (Pan troglodytes verus, Taï National Park, Cote d'Ivoire) and chimpanzees kept under semi-natural conditions (Pan troglodytes ssp., Chimfunshi Wildlife Trust, Zambia). Preliminary results propose that hand grips are similar between gorillas and chimpanzees during the manipulation of common object types. Bwindi gorillas show various hand use strategies during the processing of several plant foods. Chimfunshi chimpanzees also use forceful precision grips during daily manipulative tasks, suggesting that this is not a uniquely human ability.

\title{
Primate Trade on Java - An Overview of 25 Years of Market Surveys V. Nijman ${ }^{a}$, Wirdateti ${ }^{b}$, K.A.I. Nekaris ${ }^{a}$ \\ aNocturnal Primate Research Group, Oxford Brookes University, Oxford, UK; ${ }^{b}$ Research Centre for Biology-Indonesian Institute of Sciences (LIPI), Bogor, Indonesia E-Mail:vnijman@brookes.ac.uk
}

The exotic pet trade is one of the bigger challenges in primate conservation in Asia, and especially so in Indonesia where a wide range of primates remain popular as pets. While a significant proportion of Indonesia's primate species are protected on the country's protected species list, it is fairly common to see primates for sale at one of the many animal markets on the island of Java, Indonesia's economic and political centre. We surveyed markets for primates intermit- 
tently in the period 1994-2003 and 2012-2015, and combine these data with surveys conducted by others in the intervening period. The combined data from $>100$ surveys from the period 19912015 suggest that the diversity of species in trade has decreased significantly. The majority of the taxa traded in Java are not native to the island; the animals have been imported from Sumatra, Borneo, Sulawesi or other islands. In the 1990s and early 2000s, Bornean orang-utans, siamangs, gibbons and langurs were traded alongside macaques and slow lorises, and Pramuka market in Jakarta, in particular, stood out as a hub for trade in primates. In recent years, macaques and slow lorises have been most commonly traded, and gibbons or langurs only occasionally. Primates are less commonly traded in Pramuka (or at least less openly) and instead trade has shifted to Jatinegara market, also in Jakarta. Slow lorises, orangutans, gibbons and some langurs are protected under Indonesian law; of these protected species only the slow lorises have remained common in trade throughout the 25 -year period. Trade in non-protected macaques and langurs is subject to strict regulations - none of which the traders in the markets we monitored abided by - making the trade in all primates as observed in the markets of Java and Bail illegal. The shifts we observed over 25 years may be due to changing demands (with slow lorises having become more popular), changing availability (possibly linked to dramatic land use changes in Sumatra), or changing perceptions (with fewer people wanting to own certain primates) but is unlikely to be due to increased law enforcement as this remains virtually non-existent.

\title{
Creating Wildlife Exploration Stories to Promote Conservation of the Endangered Guizhou Snub-Nosed Monkey in Fanjingshan National Nature Reserve, China
}

\author{
Kefeng Niu ${ }^{a, b}$, Chia L. Tan ${ }^{c}$, Duoying Cuid, Shu Chen ${ }^{e}$, Lei Shi ${ }^{a}$, Emilio Balletto ${ }^{b}$, Xiaoqi Mif, \\ Marco Gamba ${ }^{b}$, Cristina Giacoma ${ }^{b}$, Yeqin Yang ${ }^{a}$ \\ aFanjingshan National Nature Reserve Administration, Tongren, China; bepartment of Life \\ Sciences and Systems Biology, University of Torino, Torino, Italy; 'San Diego Zoo Institute

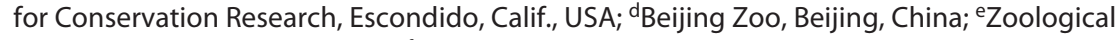 \\ Society of London, London, UK; ${ }^{\mathrm{f}}$ Tongren University, Tongren, China \\ E-Mail: newcton@126.com
}

Stories of wildlife exploration can teach children about animal biology, behaviour and habitats and serve as inspiration for them to appreciate the wonders of the natural world. Here we provide an example of a Chinese wildlife exploration storybook designed to educate children about endangered species such as the Guizhou snub-nosed monkey (Rhinopithecus brelichi), Chinese giant salamander (Andrias davidianus), mandarin duck (Aix galericulata) and butterflies (Papilio polytes and other species) of Guizhou's Fanjingshan National Nature Reserve. The book was created by a multidisciplinary group of experts made up of scientists, educators, photographers and artists. The story's central character is a fictitious wildlife enthusiast named Xinda. While exploring Fanjingshan, he befriended several scientists and learned about the biology and conservation threats of many amazing wildlife species. We will donate 4,500 copies of this book to primary schools throughout Guizhou Province, including those adjacent to Fanjingshan National Nature Reserve. Our ultimate goals through the book are to increase the knowledge about these animals and promote understanding of wildlife in schoolchildren, thereby increasing protection and sustainability of the local biodiversity. 


\title{
Gender Issues in Primate Social Signals: The Case of Yawning
}

\author{
Ivan Norscia ${ }^{a}$, Elisabetta Palagia, $b$
}

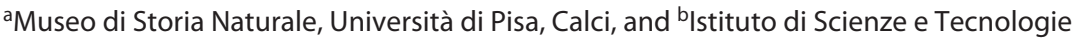

della Cognizione, Unità di Primatologia Cognitiva, CNR, Roma, Italy

E-Mail: ivan.norscia@gmail.com

Contagion yawning (yawning elicited by someone else's yawn) and spontaneous yawning are two distinct phenomena; nevertheless both can have signalling functions and be influenced by sex differences. Spontaneous yawning is possibly associated with behavioural state changes and synchronization between group members. Sex differences in yawning frequencies emerge in dimorphic species, in which males have an active role in inter-group territorial defence. In contrast, sex differences disappear in species characterized by low levels of sexual dimorphism, such as lemurs, chimpanzees and bonobos. The probability that a subject (responder) will be infected by the yawns of another subject (trigger) can be influenced by social correlates, such as the social bond between yawner and potential responder (e.g. in humans and bonobos) and rank (e.g. in chimpanzees). Hence, depending on society features (male/female dominance, type of phylopatry, presence of matrilineal subgroups, etc.), the quality and frequency of yawn contagion can differ between males and females. For example, in geladas a full matching between trigger's and responder's yawn (teeth uncovered, full open mouth, etc.) is observed only in contagion between females. In bonobos, females' yawns elicit more yawning responses than males' yawns, whereas in chimpanzees, male yawn signals trigger the highest number of responses, thus being probably more relevant to the rest of the group than female signals. In humans, it has been hypothesized that gender differences in empathic abilities may be reflected in sex differences in yawn contagion, higher between closely bonded individuals. In this work, we analyse sex differences in both spontaneous and contagious yawning across primates, with a special focus on humans.

\section{Does Primate Behavioural Flexibility Mean Long-Term Resilience in Human Landscapes?}

\author{
Katarzyna Nowak ${ }^{a, b}$, Phyllis C. Lee
}

aEvolutionary Anthropology Research Group, Department of Anthropology, Durham

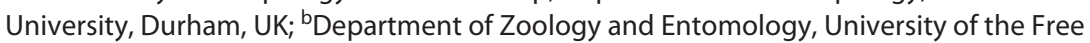
State, Qwaqwa, South Africa; 'Department of Psychology, University of Stirling, Stirling, UK E-Mail: katarzyna.nowak@durham.ac.uk

A proliferation of field studies on behavioural adaptations begets the questions: Are we seeing more examples of behavioural flexibility in nature? Is the apparent increase in reports of flexibility substantiated? Is flexibility increasingly selected for as human encroachment on wild habitats becomes more widespread? More and more, primates inhabit human-dominated landscapes: mosaics of farmland and forest, reserves with sharp edges, bases of ecotourism operations, even villages, towns and cities. Alternatively, they retreat into novel or challenging, but less disturbed habitats, such as forested swamps. A capacity for flexibility in local responses to human disturbance could buffer primates - even some so-called 'specialists' - against that disturbance. But for how long? We give examples of behavioural flexibility in the forms of dietary and habitat switching, use of invasive and non-native plant species, innovation, opportunism, novel or more frequent interspecific interactions and increased terrestriality. We propose a set of indicators including behavioural (namely perception of risk), and demographic (such as a group's structural [age-class] heterogeneity) as two measures of 'resilience' that can be used by field primatologists to monitor well-being of focal groups. We also highlight how the ability to use human-modified habitats - although an alleged determinant of extinction proneness - is not necessarily associated with any single suite of traits. The links between ecological specialization, behavioural flexibility and extinction risk in primates remain mostly uncharted.

6th European Federation for Primatology Meeting
Folia Primatol 2015;86:235-386 DOI: $10.1159 / 000435825$ 


\title{
Do Social Influences Affect Risk Preferences in Tufted Capuchin Monkeys (Sapajus spp.)?
}

\author{
Gabriele Oddi ${ }^{a, b}$, Francesca De Petrillo ${ }^{a, c}$, Emanuele Gori ${ }^{a}$, Antonia Micucci ${ }^{a}$, Fabio Paglierid, \\ Elsa Addessia \\ aUnità di Primatologia Cognitiva, Istituto di Scienze e Tecnologie della Cognizione, CNR, \\ ${ }^{\text {b}}$ Dipartimento di Biologia Ambientale, Sapienza Università di Roma, 'Dipartimento di \\ Psicologia, Sapienza Università di Roma, and ${ }^{\mathrm{d}}$ Goal-Oriented Agents Lab, Istituto di Scienze \\ e Tecnologie della Cognizione, CNR, Rome, Italy \\ E-Mail: gabryoddi@hotmail.it
}

Primates often deal with risky decisions, defined as situations with a variable rate of gains. In humans, stress level affects risk preferences, although different studies have yielded contrasting results, also depending on gender. Only one study has so far investigated how social context affects individual risk preferences in non-human primates, showing that chimpanzees and bonobos were more risk seeking when tested in a competitive situation with a human opponent than when tested in a neutral or play situation. Here, we investigate, for the first time, how the presence and the relative rank of a group mate affect risk preferences in 13 pairs of capuchins. They faced choices between a constant option (four food items) and a variable option (one or seven food items with $33 \%$ chance of getting the larger quantity) in three conditions: alone, paired with a subordinate bystander, and paired with a dominant bystander. We scored scratching, alarm call and urine washing episodes shown by both subjects to evaluate their stress level. We expected capuchins' risk preferences to change according to stress level, which is known to vary depending on the presence and the relative dominance rank of the bystander. However, given the inconsistencies in the human literature, we do not have directional predictions. Preliminary results on three female subjects, all paired with a dominant bystander, showed that in the Bystander condition subjects decreased their preference for the risky option and increased alarm calls over time, whereas in the Alone condition their performance did not vary and alarm calls decreased over time. Alarm calls produced by the bystander increased over time, positively predicted alarm calls produced by the focal subject and negatively predicted its choice for the risky option. Thus, although preliminary, our findings showed that stress level induced capuchin females to make more advantageous choices, as happens in women tested in the Iowa Gambling Task.

\author{
Infanticide Triggers Monogamy in Primates \\ Kit Opie \\ University College London, London, UK \\ E-Mail: kit.opie@ucl.ac.uk
}

Monogamy is common in birds but rare in mammals, because internal gestation and lactation in mammals encourages males to seek additional mating opportunities elsewhere. Three hypotheses have been proposed to explain the evolution of monogamy among mammals: as a male mate-guarding strategy, because of the benefits of bi-parental care, or as a defence against infanticide. So far however, comparative analyses have been unable to identify the cause of monogamy. Primates are unusual among mammals because monogamy has evolved in all the major clades and is present in $30 \%$ of extant species. By combining trait data across 230 primate species with Bayesian phylogenetic analyses it has been possible to test for correlated evolution between mating systems and a number of traits to evaluate the differing hypotheses. I will show evidence for correlated evolution between mating systems and both female ranging patterns and bi-parental care, but the most compelling explanation for the appearance of monogamy is male infanticide. Monogamy evolves only in the presence of infanticide, but once it is established there is a 
subsequent shift to paternal care and discrete female ranges. The evolution of primate monogamy can be explained by long lactation periods, due to slow development, making primate infants vulnerable to infanticide by males. I will also show evidence that bi-parental care shortens lactation periods, which reduces infanticide risk while increasing reproductive rates. These analyses support a key role for infanticide in the evolution of primate social systems.

\title{
The Evolution of Social Relationships among Primate Males Julia Ostner \\ Department of Behavioural Ecology, University of Göttingen, Göttingen, Germany E-Mail: jostner@gwdg.de
}

While the evolutionary factors driving the variation in social relationships among female primates have been thoroughly conceptualized and repeatedly subjected to empirical testing, considerably less is known about the evolutionary drivers of male social relationships. A decade ago we initiated a study on the behavioural ecology of Assamese macaques (Macaca assamensis) at the Phu Khieo Wildlife Sanctuary, Thailand, with the specific goal to further our understanding of drivers and consequences of male cooperation and social bonding. Starting with the intensity of male competition, we found that in this highly seasonal reproducing species females conceal ovulation and confuse paternity, effectively lowering male monopolization potential. Consequently, male mating and reproductive skew is very low in Assamese macaques at Phu Khieo. Consistent with theoretical models of male coalition formation, which predict with decreasing contest potential an increase in risky (all-up) coalition formation (and consequently also of protective (all-down) coalitions), male Assamese macaques formed coalitions to acquire and maintain high dominance rank. Choice of allies for coalitions was linked to patterns of dyadic male affiliation, possibly because rank changing coalitions need some degree of reliability and predictability of partners. Affiliative relationships in these dispersing males were differentiated, with stronger relationships being more symmetric in exchanges and more stable over time. These social bonds may serve several benefits, as (i) they may be the underlying, cognitively simple mechanism underlying partner choice in coalitions, (ii) having social bonds may allow males to remain in their social group after losing high status and to invest in their genetic offspring as an alternative means to increase reproductive success in a low skew species and (iii) social bonds may facilitate parallel dispersal and subsequent integration into a new group. We supplement our data on Assamese macaques with recent results on wild Barbary macaque (M. sylvanus) cooperation and bonding and integrate these findings into a more comprehensive picture across male primates.

\section{'Childrenzees': Similarities between Chimps and Children Hand Preference in a Simple Task}

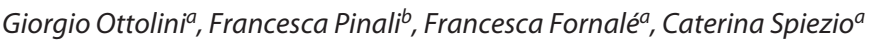 \\ aSettore Ricerca e Conservazione, Parco Natura Viva - Garda Zoological Park srl, \\ Bussolengo, and bUniversity of Turin, Turin, Italy \\ E-Mail: giorgio.ottolini@parconaturaviva.it
}

Handedness is considered to be the most obvious behavioural asymmetry in humans and could be related to a lateralization of brain functions. Despite extensive studies, its origin in the evolution of primates is still unclear: data on non-human primates have revealed that there seems to be no hand preference at the group level. In particular, for simple tasks like reaching for food,

6th European Federation for Primatology Meeting
Folia Primatol 2015;86:235-386 DOI: $10.1159 / 000435825$ 
no hand preference was reported for chimpanzees, suggesting that different factors could affect hand preference in primates, including posture, experience and task complexity. The aim of this study was to look for evidences of handedness in the simple and familiar actions of food retrieval in two species: humans and chimpanzees. The subjects of this study were 20 children (Homo sapiens) housed at the nursery 'Il Germoglio' (Bussolengo, Verona) and 14 common chimpanzees (Pan troglodytes) housed at Parco Natura Viva (Bussolengo, Verona). Both species were provided with napkins filled with a food reward. Interactions with napkins were video-recorded in order to collect data on hand use within the social context of the subjects. Results indicate that, at the group level, children show no hand preference during the interaction with the target. At the individual level, however, hand preference varies across individuals. Chimpanzees show a similar pattern, as no group level hand preference for the interaction with the target was found, whereas evidence of hand preference at the individual level was reported. These results seem to suggest that hand preference in humans and chimpanzees does not appear for simple and unimanual actions, like food retrieval. To our knowledge, this is the first study investigating hand preference in humans and chimpanzees by using the same method, adding new evidence on the evolution of this puzzling trait. This research complied with the European Directive 2010/63/EU Guidelines for the Use of Nonhuman Primates in Research.

\section{When Play Is a Strategy Game: An Overview in Adult Primates Elisabetta Palagi}

Museo di Storia Naturale, Università di Pisa, Pisa, and ISTC, Unità di Primatologia, Roma, Italy

E-Mail: elisabetta.palagi@unipi.it

A strategic game can be defined as a game in which players' autonomous decision-making skills have a high significance in determining the outcome. Although the definition is clearly designed for humans, it can also apply to some kinds of animal play, at least under some peculiar circumstances. Different from immature play, which has its main role in developing physical, cognitive and social skills, adult play is strategically employed to reach different goals depending on the social and environmental conditions in which the player acts. Playing with unrelated infants represents a social bridge which adults can use to broaden and strengthen their social networks. Cross-species comparison of the genus Macaca, characterized by different degree of tolerance, shows that the bridge strategy is particularly effective in tolerant species in which the social canalization of infants by the mothers is less severe and infants are allowed to interact playfully with other adults. Play involving only adults opens further scenarios regarding the potential roles of this versatile behaviour. Adult-adult play in primates is far more pervasive than previously thought and it seems to be a response to an immediate or delayed necessity which lies outside the play session itself. Adult play in lemurs and bonobos can serve as an ice-breaker during intergroup social encounters or as a means for overtaking unexpected situations. In apes, play can act as a form of social currency that can rapidly shape social relationships as it occurs around feeding time. The more the play, the more the cofeeding. A strategic game, worthy of its name, must rely on a complex communication based both on intentional and emotional components. In the same way, play in primates is a fertile field to explore how signals are integrated into complex and multimodal systems. In this view, play provides a window into the study of social cognition, emotional regulation and the evolution of communicative complexity. 


\title{
Maintaining Homeostasis in the Group: A Possible Role of Agonistic Support in Geladas
}

\author{
$V_{\text {Virginia Pallante }}^{a, b}$, Roscoe Stanyon ${ }^{a}$, Elisabetta Palagi ${ }^{b, c}$ \\ ${ }^{a}$ Department of Biology, University of Florence, Florence, ${ }^{b}$ Natural History Museum, \\ University of Pisa, Pisa, and 'Institute of Cognitive Sciences and Technologies, CNR, \\ Rome, Italy \\ E-Mail: virginia.pallante@studenti.unipr.it
}

Agonistic support occurs when a bystander intervenes in an ongoing conflict to help one of the two participants. In addition to the clear benefit to the recipient, agonistic support can provide benefits to the bystander who intervenes (e.g. help gain access to resources or maintain hierarchical status). However, the consequences of agonistic support provided to the victim might be different from that given to the aggressor. We hypothesized that agonistic support for the victim may not only provide a protective function for the supported subject, but also have a homeostatic role limiting the escalation of aggression among group members. To test this hypothesis, we selected Theropithecus gelada, a highly social species well equipped with behavioural mechanisms aimed at maintaining cohesion in the group. Our results show that agonistic support was preferentially directed towards the victim rather than towards the aggressor. The best supporters for the victim were high-ranking individuals. Supporting victims significantly reduced the frequency of subsequent aggression both towards the victim and further aggression in the group. In contrast, support for aggressors increased the probability of further aggression. Further, the highest levels of support were provided to the victims who were unrelated and without strong social bonds to aggressors. Agonistic support may be a social tool which comes into play when affiliative post-conflict mechanisms are less likely to occur, for instance, when the two participants are not kin or friends. This peacekeeping role (both at individual and at group level) of agonistic support in geladas could be favoured by natural selection on the basis of the self-interest and motivation of the supporter. However, we cannot exclude other selective forces, especially those that are not mutually exclusive, such as kin selection or reciprocal altruism, in the evolution of this complex and variable behaviour.

\section{Spatial Frequency Processing and Global-Local Attention Bias in Tufted Capuchin Monkeys (Sapajus apella) and Humans}

\author{
Milena Palumbo ${ }^{a, b}$, Giovanna Spinozzi $^{b}$, Carlo De Lillo $^{a}$ \\ aUniversity of Leicester, School of Psychology, Leicester, UK; ${ }^{b}$ Consiglio Nazionale delle \\ Ricerche (CNR), Istituto di Scienze e Tecnologie della Cognizione (ISTC), Rome, Italy \\ E-Mail:mp296@le.ac.uk
}

Despite the anatomical and physiological similarity in their visual system, comparative studies indicate important differences in the higher visual processes of many primate species, including tufted capuchin monkeys (Sapajus apella) and humans. Humans process the global aspect of a visual scene faster and better than its local details. This pattern is reversed in several monkey species, which process the local elements better. For this study, two experiments were carried out on tufted capuchin monkeys and humans using the identity Matching-ToSample procedure with spatial frequency (SF) gratings and hierarchical patterns in order to study the relation between spatial frequency processing and global-local processing. As there is a lack of information about the SF processing in capuchins, the first experiment was designed to clarify how they process High, Medium or Low spatial frequencies (HSF, MSF, LSF). The second experiment assessed the relationship between SF processing, attention sets and globallocal processing by manipulating the attentional bias towards either HSFs or LSFs before administrating trials requiring either global or local processing. Overall, we found that capuchins 
process HSFs better than MSF/LSFs and that biasing the attention toward either HSF or LSF is effective in modulating some aspect of subsequent global-local processing. These findings are discussed in relation to the extent to which attentional mechanisms can explain some of the observed differences in global-local processing between humans and monkeys. This research complied with the International Primatological Society (IPS) Guidelines for the Use of Nonhuman Primates in Research. The geladas were tested live in semi-natural conditions, they are never food or water deprived and experiments were carried out adopting only a reward system (favourite food).

\section{Behavioural Ecology of Chacma Baboons (Papio ursinus) in Hogsback, Eastern Cape}

Lwandiso Pamla, Judith Masters, Fabien Génin

African Primate Initiative for Ecology and Speciation, Department of Zoology and Entomology, University of Fort Hare, Alice, South Africa

E-Mail: Ipamla@webmail.co.za

Baboons are among the most widespread primates in Africa because they can adapt their omnivorous diet and foraging behaviour under challenging and changing environments. They are attracted by food, shelter and water located on human-owned properties, which brings them into conflict with residents at Hogsback, South Africa, and results in them being shot, trapped or poisoned. Our hypothesis is that baboons have been living in the Hogsback area for an extensive time period; they have adapted their foraging habits to the natural food sources available to them, and will invade cultivated land only when their natural resources are insufficient or no longer accessible. To test our hypothesis, we recorded activity budgets for foraging and movement in two troops (Ivy's troop and Nola's troop) of chacma baboons (Papio ursinus) using direct field observation: focal animal sampling and scan sampling. In addition, we used GIS methods to determine home ranges, and marked food trees for later identification. Our preliminary results support the hypothesis that the baboons spend most of their foraging time (70\%) in the forest, and much less time (30\%) in cultivated land. Crops planted by humans do, however, influence their foraging behaviour. Ivy's troop has a smaller home range $\left(3.7 \mathrm{~km}^{2}\right)$ than Nola's troop $\left(4.0 \mathrm{~km}^{2}\right)$, and includes more areas cultivated by humans and yielding easily accessible food. Responses to the presence of the baboons by many of the residents - whether affluent and living on semi-urban estates or impoverished and hunting for food - do not augur well for the future of Hogsback's baboons. Affluent residents with horticultural gardens may shoot baboons when they encounter them on their properties out of fear, while rural occupants hunt baboons in their natural environment. Both of these responses can lead to reduced population sizes and the extinction of baboon troops in the Hogsback region.

\section{The Evolution of Manipulation Behaviour in Strepsirrhines \\ Louise Peckre ${ }^{a}$, Emmanuelle Pouydebat ${ }^{a}$, Anthony Herrel ${ }^{a}$, Christine Wall ${ }^{b}$, Anne-Claire Fabre ${ }^{b}$ \\ aUMR 7179 C.N.R.S/M.N.H.N., Paris, France; b Department of Evolutionary Anthropology, Duke University, Durham, N.C., USA \\ E-Mail: louise.peckre@outlook.com}

Manipulative abilities of Catarrhini (Old World monkeys) have been studied to address a variety of questions including cognitive, functional and evolutionary ones. A high variability of grip types, including some patterns originally thought to be specific to humans, has been high- 
lighted. However, despite the fact that manipulative behaviours are involved in many important activities in all primates, they remain under-studied in Platyrrhini (New World monkeys), with the exception of capuchins, and Strepsirrhini (lemuriforms and lorisiforms). The latter have even been considered as non-manipulative due to the lack of an opposable thumb and the lack of an individualization of the digits. Given that only few studies have investigated the variability in manipulative patterns in strepsirrhines, their ability to hold and manipulate objects remains poorly understood. The present study aims to determine quantitatively the different behavioural strategies adopted by lemurs in manipulative tasks. Moreover, comparative data for over 15 highly ecologically and morphologically divergent species of lemurs will be analysed in an evolutionary context and used to discuss the origins and evolution of prehensile behaviours in primates. All animal handling was performed according to protocol IACUC \#A089-14-04 (PI: A.-C. Fabre) complying with the International Primatological Society (IPS) Guidelines for the Use of Nonhuman Primates in Research.

\title{
Temporal Variation of Food Availability and Bonobos' (Pan paniscus) Feeding Ecology in a Forest-Savannah Mosaic
}

\author{
Flora Pennec ${ }^{a}$, Victor Narat ${ }^{a}$, Annette Hladik ${ }^{a}$, Constantin Lubini ${ }^{b}$, Chloé Couturier $^{a}$, \\ Jean-Christophe Bokika Ngawoloc, Sabrina Kriefa \\ aMuséum national d'Histoire naturelle, UMR7206 Eco-anthropologie et ethnobiologie,

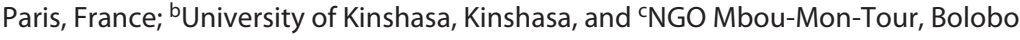 \\ territory, Democratic Republic of Congo \\ E-Mail:pennec@mnhn.fr
}

The feeding ecology of primates depends on food availability and temporal variations (seasonal and inter-annual variations) in their habitat. It is hypothesized that food availability in the distribution area of bonobos (Pan paniscus) has modulated differences in social organization (more cohesive groups, sex-roles in the bonobo society) compared to other species such as the chimpanzee (Pan troglodytes). However, there is a lack of knowledge about food availability in the extreme southwestern part of bonobo distribution. This area is characterized by a forest-savannah mosaic, an unusual habitat for bonobos. We studied food availability and the feeding ecology of a group of bonobos for 28 month (September 2012 to December 2014). This group was located in the Manzano forest, a community-based conservation area (Mbou-Mon-Tour NGO), a forest-savannah mosaic in the Bolobo territory, Democratic Republic of Congo. Food availability was determined by monitoring 26 feeding species in phenology transects $(16 \times 500 \mathrm{~m})$ and by surveying their density and basal area in 51 vegetation plots $(20 \times 50 \mathrm{~m})$. Bonobos' food items were recorded by direct observation and from fresh food remains. Different patterns of fructification were observed among the 26 feeding species. Four different fruit types could be distinguished (juicy soft pulp, juicy fibre pulp, soft aril and not fleshy) and temporal variations in fruit availability were observed during the study period. Three patterns of food consumption were observed and could be related to fruit availability. These baseline data about temporal variations of food availability for bonobos in a forest-savannah mosaic should improve our knowledge about bonobo behavioural plasticity in different habitat types. 


\title{
Seasonal Variation in the Nutritional Quality of the Diet of a Wild Population of Tufted Capuchin Monkeys in Northeastern Brazilian: The Importance of Nutrient Balance
}

\author{
Lucas Peternelli-Dos-Santos ${ }^{a}$, Patrícia Izar ${ }^{a}$, Jessica Rothman ${ }^{b}$ \\ aSão Paulo University, São Paulo, Brazil; bHunter College, City University of New York, \\ New York, N.Y., USA \\ E-Mail lucas.peternelli@gmail.com
}

Habitat quality, in terms of food availability and nutritional quality, is the main factor affecting density and behaviour of primate populations. Studies in nutritional ecology have suggested that primates seek to regulate the ingestion of a nutritional balance, rather than maximizing the ingestion of energy or of a specific nutrient. Consequently, this nutritional balance plays an important role in determining primates' diet quality. Tufted capuchin monkeys are known for their great adaptability to a wide range of different habitats. Previous studies on a wild tool using population of capuchin monkeys (Sapajus libidinosus) living at Fazenda Boa Vista (FBV), a Brazilian savannah-like environment, using estimates of fruit biomass and behavioural correlates, have suggested that monkeys do not face seasonal fruit scarcity, despite the pronounced dry season. In this study we used nutritional analysis and a geometrical approach to test whether these monkeys face seasonal differences in diet quality. From September 2012 to February 2014, we analysed a total of 169 food samples from 69 food species eaten by two groups. The food items consumed were recorded using continuous focal sampling. There were no seasonal differences in protein, carbohydrate, fibre, minerals or energy content of foods eaten. However, foods eaten during the dry season had a higher protein vs. non-protein energy ratio than the foods eaten during the rainy season. Our results suggest that, in spite of no significant seasonality in food availability, capuchin monkeys at FBV face a seasonal difference in the nutritional content of their food and, thus, in diet quality. Considering a concurrent study on nutrient consumption showing that the monkeys prioritize the ingestion of non-protein energy, we can affirm that diet quality during the dry season is relatively lower than during the rainy season. This study shows that nutrient balance is an important factor that must be considered in studies on primate feeding ecology.

\section{Underlying Mechanisms of Crop-Raiding by Chacma Baboons (Papio ursinus): Understanding Crop-Raiding Strategies and Collective Moves in Order to Control Them}

Odile Petit ${ }^{a, b}$, Hervé Fritz ${ }^{c}$, Tommy Gaillard ${ }^{a, c}$, Chloé Guerbois ${ }^{c, d}$

aPHC, UMR7178, CNRS - Strasbourg University, Strasbourg, France; bSocial Ecology Unit, Free University of Brussels, Brussels, Belgium; ' LBBE, UMR 558 CNRS - University Claude Bernard, Lyon, France; ${ }^{\mathrm{d} S u s t a i n a b i l i t y ~ R e s e a r c h ~ U n i t, ~ N e l s o n ~ M a n d e l a ~ M e t r o p o l i t a i n ~}$ University, George, South Africa

E-Mail: odile.petit@iphc.cnrs.fr

Promoting human-wildlife coexistence in the vicinity of protected areas is a complex challenge to tackle. In Zimbabwe, baboons can cause considerable damage to fields and farmers struggle to cope with this problem. Crop-raiding by baboons is a major source of conflict that negatively affects human livelihoods and wildlife conservation. The failure to manage this highlights the importance of understanding the behavioural and ecological mechanisms driving crop-raiding and the advantages of this alternative strategy, in comparison with a forest-foraging strategy, if we wish to develop an effective mitigation tool and ensure the long-term pres- 
ervation of wildlife living alongside human beings. Our study thus aimed to understand the underlying mechanisms of raiding in a troop of 40 chacma baboons (Papio ursinus) at the periphery of Hwange National Park. Direct observations conducted in April and May 2014 allowed us to collect data on 110 crop-raiding events by baboons. We identified that farms close to baboon sleeping-sites were particularly vulnerable to crop-raiding, with raids starting at dawn and stopping early in the afternoon. Results revealed that the mean number of raiders was a small proportion of the social group ( 9 out of 40 ). Moreover, male baboons raided crops more than females and exhibited more risk-prone behaviours. We also observed a gradient of propensity to lead a raid across age-categories in baboons, with adults predominantly leading. Our findings support the theory that raiding behaviour results from a trade-off where the benefit of accessing human resources outweighs potential costs. Pinpointing the drivers of this behavioural adjustment in anthropogenic areas is crucial for long-term coexistence. At a local scale, this could lead to a significant decrease in retaliatory killing and thus preserve wildlife outside protected areas. Our research complied with the IPS Guidelines for the Use of NHP in Research.

\section{Nighttime Noises: Nocturnal Wild Chimpanzee and Orangutan Calling Behaviour Using Passive Acoustic Monitoring}

\section{Alex K. Piel ${ }^{a}$, Brigitte Spillmann ${ }^{b}$, Amanda Hoepfner}

aDivision of Biological Anthropology, Department of Archaeology and Anthropology, University of Cambridge, Cambridge, UK; ${ }^{\mathrm{b}}$ Anthropological Institute and Museum, University of Zurich, Zurich, Switzerland; ' Department of Anthropology, University of Utah, Salt Lake City, Utah, USA

E-Mail:akp34@cam.ac.uk

Numerous diurnal species across taxa exhibit dawn and dusk peaks of calling, with callers exploiting low abiotic noise levels and ideal microclimates for long distance sound transmission. Nocturnal animals also respond acoustically to the environment, with well-documented lunarphilia and -phobia permeating discussion of prosimian call behaviour. However, to date the nocturnal behaviour of diurnal primates has been poorly documented, likely because of paucity in effort, rather than in behaviour. We used passive acoustic monitoring (PAM) to assess the nocturnal call activity of three populations of wild great apes: chimpanzees (Pan troglodytes schweinfurthii) from the Issa Valley, Tanzania, and orangutans (Pongo pygameus) from Tuanan and Sebangau, Indonesia. We investigated call presence as a function of moon phase, as well as other environmental factors that influence sound transmission (temperature, humidity) to test hypotheses of when individuals produce long calls during the night. Our results reflect the first systematic investigation of night-time call patterns in wild apes, and whether apes conform to the acoustic adaptation hypothesis... even when researchers sleep. We conclude by discussing the social implications of nocturnal call behaviour and the advantages of employing PAM to answer questions otherwise prohibitive to science using traditional methods. 


\section{Influence of Trichromatic Colour Vision on Children's Food Choice}

Francesca Pinali ${ }^{a}$, Caterina Spiezio ${ }^{b}$, Cristina Giacoma $^{a}$

aDepartment of Life Science and Biological Systems, University of Turin, Turin, and

${ }^{b}$ Research and Conservation Department Parco Natura Viva, Bussolengo, Italy

E-Mail: pinali.f@gmail.com

There is a considerable variability in terms of colour perception between different organisms. Primates, including humans, are the only mammals to have trichromatic colour vision. There are two different hypotheses on the explanation of the selective pressure that has led to the evolution of trichromatic colour vision in primates. The first one, called the foraging hypothesis, argues that the ability to discriminate colours has brought substantial benefits for trichromatic individuals to distinguish ripe fruit and young leaves in vegetation. The second hypothesis links trichromatic vision to the socio-sexual intraspecific communication, suggesting it provides benefits in recognition of important socio-sexual signals emitted by conspecifics. The aim of this research was to explore whether the foraging hypothesis could explain the selective pressure for the evolution of human trichromacy by observing children's preference in a 'foraging setting' in which they could choose red or green objects containing the same food reward. A group of 20 children, between 25 and 40 months of age, at the nursery 'Il Germoglio' (Bussolengo, Verona), were involved in the experimental test using red and green stimuli filled with the same food reward. Interactions with stimuli were observed in order to collect colour preference. The results indicate that, at group level, children did not show any significant colour preference for red or green objects. However, at individual level, a colour preference for red or green stimuli was found. These findings do not seem to support the foraging hypothesis according to which a group level red choice was expected, on the other hand the socio-sexual communication hypothesis cannot be excluded, as the social context could be involved in individual colour preference. In conclusion, new data have been added to the debate regarding the evolution of trichromatic colour vision, but it, however, remains unresolved.

\section{Non-Human Primates in Laboratory Research: Against Simplicity \\ Simone Pollo ${ }^{a}$, Marta Borgi ${ }^{b}$, Laura Fasano $^{a}$, Augusto Vitale ${ }^{b}$ \\ ${ }^{a}$ Sapienza Università di Roma, and ${ }^{b}$ Istituto Superiore di Sanità, Rome, Italy \\ E-Mail:vitale@iss.it}

The ethics of animal experimentation is a widely debated topic and in public discussion opinions tend to be polarized into 'against' and 'pro' views. At the same time, the academic debate does not seem less radicalized and appears somehow simplistic. The most influent animal ethics theories aim at resolving the moral problems of experimentation on animals by means of applying one single principle to it (i.e. the principle of equal consideration of suffering in Singer's utilitarianism). Likewise, supporters of animal research defend it by referring to analogous simple principles (like the sovereign good of humanity). This kind of discussion is deeply inadequate and unsatisfying: animal research is a multifaceted practice embedded in the scenario of the whole of human/animal relationships. The attempt to 'solve' the moral problem of animal research by reducing it to just simple moral calculations, or making an appeal to general principles misrepresents the complexity of moral reality. In this context, the case of non-human primates (NHP) is very interesting. Although these animals represent a minimal percentage of the total of animals used in research, they cause particular ethical concern. NHP are a special case in the laws regulating animal research. In discussing NHP as a case study, our aim will not be to suggest a different theoretical approach to evaluate the moral rightness or wrongness of experimentation on animals per se. Our attempt will be to foster a different style of discussion (academic and public) on such 
a topic, taking into account also the opinion on the use of NHP in research expressed by researchers during a series of interviews on animal experimentation. Such a case is particularly interesting since the perception of NHP at the border of 'humanity' and 'animality' challenges the tendency to solve the issues of animal research by means of an appeal to general principles and theories. Case by case analysis seems more apt to fulfil the task.

\title{
Searching for Cryptic Species: An Integrative Approach to Species Delimitation in Galagids (Primates: Galagidae)
}

\section{Luca Pozzi}

Behavioural Ecology and Sociobiology Unit, German Primate Centre, Leibniz Institute for Primate Research, Göttingen, Germany

E-Mail: Ipozzi@dpz.eu

Describing species biodiversity is one of the main goals in evolutionary primatology. This is a critical task for exploring modes of speciation and for conservation biology. However, identifying species boundaries has proven to be particularly challenging for cryptic organisms where only subtle morphological variation is present. Research on cryptic species has recently increased exponentially due to the availability of molecular techniques and it has been suggested that biological diversity among these species is possibly much higher than expected based on purely morphological basis. However, over the last decade, the (ab)use of molecular tools led some researchers to suggest that the increase in species number is more a consequence of taxonomic inflation than actual diversity. Nocturnal galagids are among the most cryptic of all primates and - due to the lack of genetic data - species diversity has been described based mainly on acoustic behaviour leading to a controversial increase in the number of recognized species. The aim of this study is to investigate species boundaries in a highly cryptic complex, the Zanzibar dwarf galagos (genus Galagoides), using an integrative approach. First, I use multilocus genetic data in a two-step approach including discovery and validation methods under a coalescence framework. I then compare the genetic results with bioacoustic data and species distribution models to explore the mechanisms involved in reproductive isolation and speciation. The results suggest that $G$. cocos and G. zanzibaricus represent two valid cryptic species that probably underwent speciation in the Mid-Pliocene ( $3.5 \mathrm{Ma}$ ) while fragmented in isolated populations in the Eastern African forests. Finally, I discuss the pros and cons of molecular methods and the importance of integrating multiple lines of evidence in delimiting species. This research complies with the International Primatological Society (IPS) Guidelines for the Use of Nonhuman Primates in Research.

\section{Great Apes on the Road towards Extinction and International Protective Legislation}

\author{
Signe Preuschoft \\ Compentence Centre Apes, Vier Pfoten, Vienna, Austria \\ E-Mail: signe.preuschoft@vier-pfoten.org
}

The causes of the dramatically diminishing numbers of great apes vary. In Africa the bushmeat trade and human transmitted diseases pose serious risks, but in Asia large scale habitat destruction is the driving force. In both regions, extractive industries and the globalised world's insatiable hunger for energy cause great ape habitats to disappear. Two thirds of great ape range countries are among the poorest of the world. Their right to economic development cannot be denied. Developed nations are loath to endanger economic growth but NGOs can target con- 
sumer behaviour and companies' corporate ethics in these markets. However, markets of threshold economies become ever more important and offer virtually no points to lever consumer control. If current trends continue, by 2032 : $99 \%$ of the orangutan range will suffer medium to high impacts from human development, as will $90 \%$ of the gorilla range, $92 \%$ of the chimpanzee range and $96 \%$ of bonobo range (Caldecott \& Miles 2005). Opening up ape habitat entails infrastructure which in turn aggravates human immigration and human-ape conflict. National laws banning the capture, killing and trading of apes, alive or dead, are rarely enforced. While grown apes are killed, their orphans are often kept alive for the illegal pet trade. The Convention on International Trade in Endangered Species of Wild Fauna and Flora (CITES) is a powerful tool to curb international trade of endangered species such as great apes. Yet if no mechanisms are in place to regulate what will happen after an ape has been confiscated in its home nation, its wellbeing and life are still in jeopardy.

\title{
Investigating the Human-Animal Relationship between Zoo-Housed Western Lowland Gorillas (Gorilla gorilla gorilla) and Their Keepers
}

\author{
Elena Račevska, Catherine M. Hill
}

Oxford Brookes University, Oxford, UK

E-Mail: elenaracevska@gmail.com

The human-animal relationship (HAR) is defined as the relationship between a human and an animal that have a history of interactions, which allows them to make predictions about each other's behaviour. Differences in animal handling, management and housing have been found to result in positive, neutral or negative HARs, and positive HARs facilitate husbandry and promote good welfare. Using the relationship between western lowland gorillas (Gorilla gorilla gorilla) and their keepers in two BIAZA-accredited zoos, we examine how animal and keeper characteristics influence HARs (henceforth referred to as keeper-animal relationships, KARs) in a zoo environment. We adopt a mixed-methods approach to access information about KARs. Semi-structured interviews are used to collect data on animal (e.g. age, sex, rearing history) and keeper (e.g. work experience, education, husbandry style, age) variables and their potential contribution to KARs; an independent KAR measure is obtained through systematic observations of keeper-gorilla interactions. The 'Gorilla Behaviour Index' is used to obtain keepers' ratings of gorillas 'personalities'. Additionally, instantaneous scan sampling is used to record animal activity budgets, providing a welfare measure. We use the results to explore how keepers perceive gorillas in their care and discuss how those perceptions contribute to KARs. By gaining a better understanding of the effects that KARs play in welfare, we may begin incorporating this knowledge into development of future captive primate care protocols. Research complied with the International Primatological Society (IPS) Guidelines for the Use of Nonhuman Primates in Research.

\section{Variable Female Power in Mouse Lemurs or 'What Do They Keep on Fighting for?'}

\author{
Ute Radespiel, Sarah Hohenbrink
}

Institute of Zoology, University of Veterinary Medicine Hanover, Hannover, Germany

E-Mail: ute.radespiel@tiho-hannover.de

Power disparity between the sexes is well known from many mammalian species with male dominance as the predominant pattern and female dominance being the exception. However, female dominance seems to be rather widespread among lemurs. Despite various efforts, no sin- 
gle selective force has yet been identified that explains female power in lemurs. What if there is no single explanatory factor? Extant lemur species may have inherited this trait from their common ancestor, but may have lost or retained it for different ultimate reasons. The comparison of the expression of female dominance in closely related congeners can help to illuminate possible evolutionary drivers of this diversity. As a first step, we present comparative data obtained with a standardized experimental encounter paradigm that was used to investigate intersexual dominance pattern in the field and under captive conditions in three different mouse lemur species, the smallest nocturnal solitary foragers among Malagasy lemurs. The three study species differed in conflict rates, the proportion of decided conflicts and the power disparity between the sexes. Whereas female dominance could be confirmed in the grey mouse lemur (Microcebus murinus) and the Goodman's mouse lemur (M. lehilahytsara), no evidence of female power was detected in the golden-brown mouse lemur (M. ravelobensis). A review of the available data on the ecology and sociality of these species suggests that these differences in intersexual dominance relationships are more likely driven by socioecological differences and more specifically by speciesspecific sleeping-site requirements than by different food-related energetic constraints. The implications of this new functional hypothesis for the understanding of female dominance in lemurs are discussed. This project complied with the European Directive 2010/63/EU and IPS Guidelines for the Use of Nonhuman Primates in Research, and experiments were approved by the appropriate governmental institution (LAVES, Az. 33.9-42502-05-10A08).

\section{Distribution of Paternity within a Large, Semi-Captive Group of Japanese Macaques (Macaca fuscata)}

Claudia Radler, Elfriede Kalcher-Sommersguter, Kristina M. Sefc, Cornelia Franz-Schaider

University of Graz, Graz, Austria

E-Mail: claudia.radler@edu.uni-graz.at

Japanese macaques (Macaca fuscata) live in female-bonded groups containing several female matrilines and, generally, unrelated males, which are the dispersing sex. We investigated the distribution of paternity in a large semi-captive group of Japanese macaques $(n=140$ individuals). The group originates from a founder population of 40 individuals, which were imported to Landskron (Austria) in 1996, and no transfer of individuals has occurred since then. Therefore, we assume a high risk of inbreeding, which leads to the question of whether any countermeasures are taken to lower the possible disadvantageous effects of this risk. Furthermore, we were interested in the dominance status of fathers. Paternity was identified for 43 individuals out of a total of 85 descendants and data showed that reproduction among closely related individuals was as frequent as expected by chance (permutation tests, n.s.). However, analysis of behavioural data collected during the breeding season showed that females significantly avoid sexually motivated contact with closely related males $(\rho=-0.692, p=0.004, n=15)$ but not vice versa $(\rho=-0.202$, n.s., $\mathrm{n}=18$ ). This may represent a behavioural mechanism to avoid inbreeding among closely related mating partners on the part of the females which - in the light of at least one observed kin mating - does not appear to be perfectly efficient. Furthermore, the results showed that high ranking males were not able to monopolize paternity, probably due to the high ratio of adult males to females in this population.

6th European Federation for Primatology Meeting
Folia Primatol 2015;86:235-386 DOI: $10.1159 / 000435825$ 


\title{
Conservation Physiology of Two Sympatric Lemur Species: Is the Specialist More Vulnerable to Habitat Degradation?
}

\author{
Hasina Josué Rakotoniaina ${ }^{a}$, Peter Kappeler ${ }^{a, b}$, Cornelia Kraus $^{a}$ \\ aDepartment of Sociobiology/Anthropology, Faculty of Biology, University of Göttingen, \\ and ${ }^{\mathrm{b}}$ Behavioural Ecology and Sociobiology Unit, German Primate Centre, Göttingen, \\ Germany \\ E-Mail: hjrakotoniaina@dpz.eu
}

Understanding how animals react to human-induced changes in their environment is pivotal to conservation biology, but, according to their position along the specialist-generalist continuum, species are believed to react differently to changes in environmental condition, with specialists usually being considered more vulnerable. In this ongoing project, we compare a set of physiological parameters in two lemur species across four habitats which can be ordered along a gradient of human disturbance. Cheirogaleus medius is a specialist, and, at Kirindy Forest, western Madagascar a rapid local population decline has been observed, while numbers of the more generalist, sympatric Microcebus murinus have increased. Hence, these two closely related species seem to be suitable candidates for a vulnerability assessment study with respect to habitat deterioration. Our specific project aims are to (i) relate habitat factors that are likely affected by human influence to several health indicators such as body condition, parasite load and stress level, and (ii) to investigate the potential effect of these health indicators on short term fitness components such as survival and recruitment. To this end, information on habitat factors know to be important for both species such as food and shelter trees density and shelter insulation capacity together with parasitological and endocrine (hair cortisol levels) data were collected over two consecutive years. Biological samples were gathered during monthly routine capture and this protocol facilitated the record of demographic data. Preliminary results indicate a positive correlation between the parasite prevalence in C. medius and the degree of disturbance of the habitat, with a two-fold difference between the least and the most disturbed habitat. In contrast, $M$. $m u$ rinus shows highest parasite prevalence in the least disturbed site, where it occurs in high densities. Approval for research, capture and sample export have been obtained without problems from the responsible Ministry for the Environment, Water and Forests in Madagascar as well as the German Federal Nature Conservation Agency (Bundesamt für Naturschutz).

\section{Speciation in Mouse Lemurs: Clues to High Levels of Diversity in Malagasy Strepsirrhines}

Hajarimanitra Rambeloarivony, Fabien Génin, Judith Masters

African Primate Initiative for Ecology and Speciation (APIES), University of Fort Hare, Alice, South Africa

E-Mail: haja.kely2010@gmail.com

High level of diversity characterise the Malagasy strepsirrhines, Lemuriformes. The combination of physical barriers and ecological shift during the quaternary paleoclimatic variation were proposed as the sources of such high diversity. However, the process and mechanisms that lead to such diversity are still poorly understood. The Recognition Concept (RC) of Hugh Paterson suggests that new species form only when a notable ecological shift has effected sufficient environmental change to disrupt the function of the Specific-mate Recognition System (SMRS). The SMRS comprises communication signals including odours, vocalizations and visual signals. The SMRS is therefore considered not only to be species-specific, but adapted to function within the preferred set of environmental conditions that initially drove the species' emergence. Using the reproductive calls of two closely related species of mouse lemurs (Microcebus griseorufus and M. 
murinus), we tested the propagation of the specifically distinct but superficially similar male mating calls of these species in different habitats (i.e. spiny forest: the natural habitat of M. griseorufus, and gallery forest: the natural habitat of $M$. murinus). We will try to explain the biogeography of these two mouse lemur species in terms of the propagation properties of their habitats using the bioacoustics of habitat structure as a main predictor. We suggest that the inability to communicate properly in a given habitat limit the biogeography of each studied species. We will discuss the importance of SMRS in primate phylogeny, taxonomy, speciation and conservation. This study complies with the European Directive 2010/63/EU and the International Primatological Society Guidelines for the Use of Nonhuman Primates in Research and the Malagasy and the University of Fort Hare regulations regarding the ethical treatment of research subjects.

\section{Two Tasks, One Hand: Hand Preference in Old World Monkeys}

Barbara Regaiollia ${ }^{a}$ Paola M. Valsecchib ${ }^{b}$ Caterina Spiezio $^{a}$

aResearch and Conservation Department - Parco Natura Viva, Bussolengo, and buniversity of Parma, Parma, Italy

E-Mail: barbara.regaiolli@parconaturaviva.it

The study of manual lateralization in non-human primates could be an optimal way to trace the evolution and adaptive value of handedness in our species. This research aims at investigating hand preference in Old World monkeys during simple and complex tasks, considering the effect of sex and age on primate handedness. Two different studies were carried out: a low level task, in which data about simple and spontaneous actions (foraging, manipulation and locomotion) were collected and a high-level task, in which hand preference during the interaction with an experimental apparatus (a thick paper tube containing food rewards) was recorded. The low-level task was carried out with 7 pig-tailed macaques (Macaca nemestrina), 16 Barbary macaques (M. sylvanus) and 9 vervet monkeys (Chlorocebus aethiops), whereas the high-level task involved 15 Barbary macaques and 8 vervet monkeys. Monkeys were tested in their social context, using noninvasive techniques. In the low-level task, a significant group level, right hand preference was found. In particular, a strong bias toward right hand use for starting locomotion was reported. Regarding the high-level task, no significant group level hand preference was found for any of the actions considered. However, when comparing the hand preference between the low-level and the high-level task, the strength of the hand preference increases along with the complexity of the action. These results suggest possible implications of factors such as posture and task complexity in the evolution of manual lateralization. The effect of age and sex on Old World monkey hand preference will be also discussed. This research complied with the European Directive 2010/63/ EU Guidelines for the Use of Nonhuman Primates in Research.

\section{Relationships between Altitude, Habitat Structure and Behaviour of Nycticebus javanicus in a Submontane Agroforest}

Kathleen D. Reinhardt ${ }^{a}$, Wirdateti ${ }^{b}$, K.A.I. Nekaris ${ }^{a}$

${ }^{a}$ The Little Fireface Project, Nocturnal Primate Research Group, Oxford Brookes University,

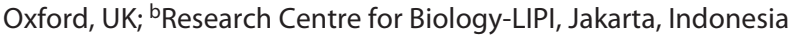

E-Mail: Kathleen.Reinhardt-2015@brookes.ac.uk

Joint impacts of anthropogenic disturbances and climate change are of pressing concern for modern conservationists. If global warming patterns and deforestation increase, issues of population density and biodiversity may greaten, forcing primate species to either shift home ranges to 
higher elevations with cooler temperatures, or overlap with agricultural plantations in locally owned forest areas. Listed as Critically Endangered and included in the Top 25 Most Endangered Primates for a fourth consecutive time, the Javan slow loris (Nycticebus javanicus) finds itself increasingly affected by similar environmental constraints. With less than $10 \%$ of rainforests remaining in Java, Indonesia, this endemic species is forced to range across highly fragmented agroforest areas or at altitudes above $800 \mathrm{~m}$ asl. Through use of radio tracking, vegetation plots, behavioural observations and weather station loggers, we analysed the effects of climate and habitat structure on the behaviour and microhabitat use of wild $N$. javanicus social pairs ranging at varying altitudes $(1,000,1,300$, and $1,500 \mathrm{~m}$ asl $)$ in the submontane agroforests of Cipaganti, Garut region, West Java. Using general linear mixed models and stepwise regression analyses in SPSS 21, we found forest connectivity and altitude to be significant predictors of $N$. javanicus behavioural patterns (d.f. $=2, \mathrm{~F}_{\text {ratio }}=3.7, \mathrm{p}<0.041$; d.f. $=1, \mathrm{~F}_{\text {ratio }}=4.93, \mathrm{p}=0.04$ ). Use of substrate type and size for specific loris behaviours varied between microhabitats, with a tendency to use terminal substrates for foraging and feeding at higher altitudes and larger trees for travelling behaviours in all microhabitats. From these results, we present a population management plan for wild $N$. javanicus with regard to climate change scenarios in a highly disturbed agroforest area - a process characterising much of the remaining geographic range of all eight slow loris species.

\title{
Female Japanese Macaques Advertise Pregnancy in Multiple Modalities
}

\author{
Lucie Rigaill ${ }^{a}$, Andrew JJ. MacIntosh ${ }^{a, b}$, James P. Higham ${ }^{c}$, Sandra Winters ${ }^{c}$, Keiko Shimizu ${ }^{d}$, \\ Keiko Mouri ${ }^{a}$, Takeshi Furuichi ${ }^{a}$, Cécile Garcia ${ }^{e}$ \\ aSocial Systems Evolution Section, Department of Ecology and Social Behaviours, Primate \\ Research Institute, Kyoto University, Inuyama, and 'bildlife Research Center, Kyoto \\ University, Kyoto, Japan; 'Department of Anthropology, Center for the Study of Human \\ Origins, New York University, New York, N.Y., USA; dDepartment of Zoology, Faculty of \\ Science, Okayama University of Science, Okayama City, Japan; 'Laboratoire de Dynamique \\ de I'Evolution Humaine, UPR 2147, CNRS, Paris, France \\ E-Mail: lucie.rigaill.75w@st.kyoto-u.ac.jp
}

Female primates signal their reproductive state through behavioural, visual (e.g. swelling size), auditory (e.g. copulation calls) and olfactory (i.e. vaginal odorant compounds) cues. To date, however, studies have generally focused on ovulatory signalling, and multimodal advertisement of pregnancy is still largely uninvestigated. We aimed to determine if female Japanese macaques (Macaca fuscata) advertise their pregnancy via changes in behavioural, visual and auditory sexual signals, and the role of these signals in male mating decisions. We combined digital photography, behavioural and endocrinological (progestogen and oestrogen) data from 5 females, for three one-month periods: pre-conceptive period, first month of pregnancy and second month of pregnancy. We found that males and females did not copulate during pregnancy, and that most sexual behaviours (e.g. male and female approaches, male holding behaviours) decreased between the pre-conceptive and the pregnancy period. Female faces became significantly darker from the pre-conceptive period to pregnancy, and face redness significantly decreased between the 1st and 2nd month of pregnancy. Oestrus call frequency was significantly lower during the 1st month of pregnancy compared to the pre-conceptive period and the 2 nd month of pregnancy. Our results suggest that pregnancy in Japanese macaques is signalled multimodally (i.e. behavioural, visual and potentially auditory signals), which is likely to provide benefits to both sexes. Females and males could decrease the overall cost of mating, with females allocating their energy to foetal growth, and males focusing their reproductive effort on cycling females. The study was conducted in agreement with the International Primatological Society Guidelines for the Use of Nonhuman Primates in Research.

346
Folia Primatol 2015;86:235-386 DOI: $10.1159 / 000435825$ 6th European Federation for Primatology Meeting 


\title{
Conservation of Eulemur mongoz and Eulemur fulvus in the Comoros Islands
}

Roberta Righini ${ }^{a}$, Bakri Nadhurou $^{a}$, Marco Gamba $^{a}$, Ahmed Ouledi $^{b}$, Cristina Giacoma $^{a}$

aDepartment of Life Sciences and Systems Biology University of Turin, Turin, Italy;

bUniversité des Comores Faculté des Sciences et Techniques, Moroni, Union des Comores

E-Mail: righini.roberta@gmail.com

The Comoros Archipelago is an important biodiversity hotspot and hosts the only two lemur species found outside Madagascar, Eulemur fulvus (common brown lemur) in the island of Mayotte and Eulemur mongoz (mongoose lemur) in Moheli and Anjouan islands. The common brown lemur is classified as Near Threatened and the mongoose lemur as Critically Endangered by IUCN criteria. The aim of this study was to update information on the status and the local threats affecting these two lemur species in the Comoros islands. We surveyed the presence of lemurs and of the principal threats in the three islands. In Mayotte, we recorded the presence of 377 common brown lemurs belonging to 40 groups. They lived in three types of environment: dry forest, humid forest and anthropized areas. In Mohéli and Anjouan, we recorded 214 mongoose lemurs of 63 groups. Group size and composition depended on environmental parameters as well as both type and extent of anthropogenic pressures. Logging, slash and burn agriculture and livestock grazing were common in the islands. The reduction and fragmentation of forest areas caused a significant change in the number of groups and their size. We also recorded an increase in the presence of lemurs in agricultural and urban areas, causing conflicts especially with the local farmers. We integrated survey data with a questionnaire-based analysis by interviewing members of local communities. We found that lemurs were considered a nuisance because they feed on fruits and seeds, causing damage to farmers' incomes. The results showed that hunting lemurs for food or to defend fruit and crops is still practiced by local people. However, we have reason for hope as our study also demonstrated that educational activities are successful in promoting a positive attitude toward lemurs and conservation actions in the local population. This research complied with the International Primatological Society (IPS) Guidelines for the Use of Nonhuman Primates in Research.

\section{Can Their Sounds Find a Way? Primate Calls and Environmental Noise in China}

\author{
Isidoro Riondato ${ }^{a}, K_{\text {efeng Niu }}^{a, b}$, Marco Gamba $^{a}$, Yeqin Yang ${ }^{b}$, Cristina Giacoma ${ }^{a}$ \\ aDepartment of Life Sciences and Systems Biology, University of Torino, Torino, Italy; \\ ${ }^{b}$ Fanjingshan National Nature Reserve Administration, Tongren, China \\ E-Mail: isidoro.riondato@unito.it
}

Acoustic signals are influenced by their transmission through the environment from an emitter to the receiver(s). Study on environmental characteristics can provide relevant information about animal acoustic communication and the selection pressures that shaped the evolution of vocal signals. Animals have to limit attenuation and degradation of signal structure to maintain communicative efficacy over distance. The aim of this study is to evaluate how environmental characteristics effect the degradation and attenuation of acoustic signals emitted by Rhinopithecus brelichi. We present the results of sound transmission experiments conducted in 2013 in the sub-tropical forest of the Fanjingshan National Nature Reserve (FNNR - Guizhou Province, China). This mountainous, remote area represents the last natural area where Rhinopithecus brelich $i$ survives. $R$. brelich $i$ is an elusive and endangered Chinese endemic primate species. We broadcast and recorded a sequence of signals consisting of eight pure tones (ranging from 1,000 to $28,000 \mathrm{~Hz}$ ) and six species-typical vocalizations (previously collected from captive individuals 
at Beijing Zoo and FNNR Rescue Centre). We chose five distinct linear transects along which we conducted the experimental procedure recording the emitted sequence (at distances of 1,2, 4, 8, 16 and $32 \mathrm{~m}$ from the speaker) using two microphones placed at different heights (ground level and 2 metres up). Dissimilarity index obtained by Dynamic Time Warping (DTW) increased along the transect with distance. We replicated the procedures to analyse the effect of vegetation, daytime and seasonal variations on the quality of sound transmission. This research complied with the International Primatological Society (IPS) Guidelines for the Use of Nonhuman Primates in Research.

\section{Infrared Thermography as a Non-Invasive Method for Measuring Emotional States in Barbary Macaques (Macaca sylvanus)}

Lindsey Robbins, Julia Lehmann

University of Roehampton, London, UK

E-Mail: robbinsl@ roehampton.ac.uk

Infrared thermography (IRT) is a relatively new method that is gaining interest in the scientific community, particularly in terms of assessing the welfare of animals. Until recently, IRT was used mainly for military purposes, primarily due to the high costs of the cameras. However, with recent technological advances, cameras are now smaller and sold at more affordable prices for private use. The present study, conducted with free-ranging Barbary macaques (Macaca sylvanus) and using a Seek Thermal camera attached to a Galaxy S5 mobile device, aimed to (1) explore the potential and validity of infrared thermography using this relatively new and low-cost camera, and (2) determine if infrared thermography has the capability to detect facial temperature differences related to positive and to negative behavioural events. To determine the requirements for optimal distance from a subject for IRT measurements, thermographic images were taken at different distances from a known temperature source. Next, to test reliability, images were taken every $5 \mathrm{~s}$ of a Barbary macaque at rest to see how temperature measurements varied within subjects during a presumed constant state. Finally, to determine if IRT could be used to assess emotional states, facial temperature was measured in the periods 5 min before, during and 5 min after an emotionally positive (grooming) or negative (receipt of aggression) event took place. Results of this study will be presented in this poster, along with a discussion of the potential value of IRT in primatology. Research complied with the International Primatological Society (IPS) Guidelines for the Use of Nonhuman Primates in Research.

\section{Casting Light on Prosimian Relocations: Long-Term Monitoring of Relocated Eulemur collaris in South-Eastern Madagascar}

Marika Roma ${ }^{a}$, Fiona Rowe ${ }^{b}$, Laza Andriamandimbiarisoa', Damiano Marchia, Jean-Baptiste Ramanamanjato', Giuseppe Donatib

${ }^{a}$ Department of Biology, University of Pisa, Pisa, Italy; ${ }^{b}$ Department of Social Sciences, Oxford Brookes University, Oxford, UK; ' $\mathrm{Q}$ MM Madagascar, Fort Dauphin, Madagascar E-Mail: romarika89@gmail.com

The littoral forest in Madagascar is one of the habitats that have suffered a great reduction in size, and the vast diversity of plants and animals living in it is now highly threatened. In the south-east of the island, a few fragments of littoral forest still harbour small populations of the endangered collared brown lemur, Eulemur collaris. In 2000, the rapid devastation of one of these 
forest fragments by charcoal makers (in the area of Mandena) elicited a rescue action and relocation of 28 E. collaris into a nearby, but recently protected, fragment. Since then, some of the relocated animals have been observed by a team of observers from QMM (QIT Madagascar Minerals) in order to get insight into their adaptation to the new area. Since there is a paucity of longterm data on prosimians relocations, these monitoring efforts are crucial to understand how prosimians in general and lemurs in particular may adapt to unfamiliar areas. We analysed dietary choice in one relocated group of E. collaris throughout several years and we compared it with data from other populations to assess whether the release site provided a suitable habitat. We analysed data collected by local QMM assistants in 2007, 2008, 2009 and 2013. The analysis indicated that the diet of E. collaris remained mainly frugivorous over the years, which is the usual dietary regime shown by this species in natural situations. A Mixed Model indicated that seasonal fluctuations were wider than inter-annual differences, which is in line with the highly variable tree phonological pattern in this habitat. This multi-annual study indicated that $E$. collaris did not modify its overall diet at the release site in terms of major food categories but the use of tree species varied significantly throughout the years. Summarizing, our work suggests that Eulemur species may tolerate relocations and the disruptive effects of these operations are not apparent on the animals' behavioural ecology over the long-term.

\title{
Testing for Sexual Selection on Rhesus Macaque (Macaca mulatta) Face Shape
}

\author{
Kevin Rosenfield ${ }^{a}$, Constance Dubuc $^{b}$, Stuart Semple ${ }^{a}$ \\ ${ }^{a}$ Centre for Research in Evolutionary and Environmental Anthropology, University of \\ Roehampton, London, and ' ${ }^{2}$ arge Animal Research Group, Department of Zoology, \\ University of Cambridge, Cambridge, UK \\ E-Mail: rosenfik@roehampton.ac.uk
}

Inter-sexual selection acts on traits that influence an animal's attractiveness to the opposite sex. Testing for sexual selection requires examining whether the measured variation in the trait of interest is perceived by opposite-sex conspecifics. In this study, we test the hypothesis that male face shape in rhesus macaques (Macaca mulatta) has been driven by inter-sexual selection. We tested firstly for sexual dimorphism in face shape, and quantified variation among males in degree of facial masculinity (FM). Nine facial features (e.g. jaw width, face width, lower face height) were measured to quantify FM in 71 male and 28 female rhesus macaques living on Cayo Santiago, Puerto Rico. Unstandardized scores from multiple linear regression of facial trait sizes against sex were used as an index of FM. Analyses revealed that sex differences in FM were highly significant with very little overlap between the sexes, providing evidence for strong sexual dimorphism in face shape. FM scores were, however, highly variable among males, such that some had highly masculine faces and others more feminine faces. We are currently testing female preferences for FM using a differential looking-time experiment, carried out on Cayo Santiago. Females are being shown images of one feminine (in the bottom $40 \%$ of FM scores) and one masculine (in the top $40 \%$ of FM scores), with duration of looking at each scored from blind video recordings. The results of this study will be presented as a poster presentation at the 6 th meeting of the European Federation of Primatology. This study complies with the International Primatological Society Guidelines for the Use of Nonhuman Primates in Research. The investigation was approved by the IACUC of the University of Puerto Rico, Medical Sciences Campus (protocol No. A0100108), and University of Roehampton Ethics committee. 


\title{
Social Strategies during Male Immigration in Captive Groups of Rhesus Macaques
}

\author{
A. Rox ${ }^{a, b}$, E.H.M. Sterck ${ }^{a, b}$, L.M. van den Berg ${ }^{b}$

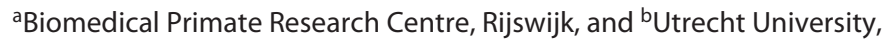 \\ Utrecht, The Netherlands \\ E-Mail:a.rox@uu.nl
}

Primate social behaviour is complex. Both human and non-human primates may cooperate, cheat, punish and coerce to gain social and fitness benefits. Up to now, research has focused on the social strategies of non-human primates in socially stable situations. However, social strategies may be even more essential in socially unstable situations since newly built social relationships will directly influence an individual's fitness. Therefore, the use of social strategies during male immigration in captive groups of rhesus macaques (Macaca mulatta) is investigated in this study. These immigrations were carried out for management reasons, complied with the European Directive 2010/63/EU, and were not experimentally induced. Observational data on affiliative, aggressive, sexual and proximity behaviour of resident and immigrant animals were collected during this study. The results indicate that males attempt to cooperate during immigration to gain access to mating partners, displaying friendly behaviour and low levels of aggression. Females, on the other hand, may differ in their approach and use a mixture of strategies: cooperation, avoidance or even displaying high levels aggression. Which strategy females use depends on individual characteristics such as fertility. This study forms a starting point in researching social strategies in socially unstable situations, and is the first step in identifying which factors determine a male's success at immigration. The outcomes of this study will not only contribute to our understanding of the evolution of human social behaviour, but can also help to improve the colony management in captive breeding facilities and zoos.

\section{Neural Basis of the Effort/Reward Trade-Off in the Ventral Prefrontal Cortex of Rhesus Monkeys}

\author{
Aurore San-Galli ${ }^{a}$, Mathias Pessiglione ${ }^{b}$, Sebastien Bouret ${ }^{b}$

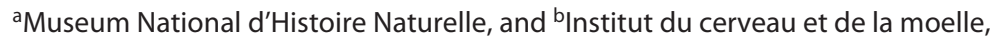 \\ Paris, France \\ E-Mail: sangalliaurore@gmail.com
}

For many primates, foraging requires individuals to trade-off between the nutritional value of food items and the foraging effort needed to locate and obtain these items. The integration of this information and the computation of the resulting value of the different options is thought to involve the ventral part of the prefrontal cortex. Our work aims at identifying the neural mechanisms that underlie the representation of goal value in the ventro-medial prefrontal cortex (VMPFC) of rhesus monkeys (Macaca mulatta). We recorded the activity of 111 VMPFC neurons in 2 macaques performing a grip force task where the amount of reward and the amount of effort required for obtaining that reward were manipulated systematically. Monkeys readily adjusted their behaviour in the task as a function of the expected outcome value, which increased with reward and decreased with effort as well as fatigue or satiety. The activity of VMPFC neurons showed relatively little response to visual information, but it was strongly modulated by internal determinants of outcome value, such as memory and satiety/fatigue. In addition, the activity of VMPFC neurons was constantly associated with the willingness to perform the task: at any point in time, the neuronal signal reflected the decision of the monkeys to engage in the task and perform the actions necessary to reach their goal. Thus, our data indicate that the firing of VMPFC 
neurons is strongly associated with internal determinants of subjective valuation and decision making. This activity might be critical for comparing information about feeding opportunities and selecting foraging paths in natural foraging situations. The research presented complies with the European directive 2010/63/EU and with the French laws (code rural, articles R. 214-87 à 214-126). It has been approved by our ethics committee and is registered at the French Ministry of Research, project number 000581.02.

\section{The Middle and Late Pleistocene Macaca sylvanus Fossil Record from Italy}

Raffaele Sardella ${ }^{a}$, Fabio Bona ${ }^{b}$, Dawid Adam lurino ${ }^{a}$, Lorenzo Rook ${ }^{c}$, Luca Bellucci $^{a}$

${ }^{a}$ Dipartimento di Scienze della Terra, Sapienza Università di Roma, Roma, ${ }^{b}$ Casalbuttano ed Uniti, and 'Dipartimento di Scienze della Terra, Università di Firenze, Firenze, Italy

E-Mail: raffaele.sardella@uniroma1.it

During the Plio-Pleistocene, the Barbary macaque Macaca sylvanus was widely distributed throughout Europe. Fossils are usually referred to the $M$. sylvanus lineage, but their specific taxonomic status is controversial because, due to its conservative morphology, it is difficult to distinguish fragmentary fossils of Macaca from other primitive papionins or define a firm alphataxonomy. Three subspecies are classically recognised in the fossil record of continental Europe: the Pliocene M. sylvanus prisca Gervais, 1859; the Late Pliocene to Early Pleistocene M. sylvanus florentina Cocchi, 1872; and the Middle to Late Pleistocene M. sylvanus pliocena Owen, 1846. The Plio-Pleistocene Italian fossil record includes cranio-mandibular fragments, isolated teeth or fragmentary postcranial bones. The best preserved and complete material comes from Villafranca D'Asti (Piedmont), Upper Valdarno (Tuscany) and Pietrafitta (Umbria). Recent studies focussed on fossil remains from Middle and Late Pleistocene deposits of Italy. This analysis includes recently discovered material and old paleontological collections. Among them is an almost complete mandible from the Roma area, which has been reconsidered and studied using CT scanning and computer graphic methodologies. Intraspecific variability, biochronology and ecology of the Pleistocene Barbary macaques are discussed together with the possible causes of their extinction in Europe.

\section{Reach-to-Grasp in Naturalistic Settings: A Kinematic Approach \\ Luisa Sartori ${ }^{a, b}$, Andrea Camperio-Ciania ${ }^{a}$, Maria Bulgheroni ${ }^{c}$, Umberto Castiello $^{a, b, d}$ \\ aDipartimento di Psicologia Generale, Università di Padova, and ${ }^{b} C e n t r e$ for Cognitive Neuroscience, Università di Padova, Padova, ' ${ }^{\mathrm{C}}$ b. Acus., Milano, and ${ }^{\mathrm{d} C e n t r o ~ B e n i a m i n o}$ Segre, Accademia dei Lincei, Roma, Italy \\ E-Mail: luisa.sartori@unipd.it}

The prehensile hand is one of the major traits distinguishing primates from other mammal species. All primates, in fact, are able to grasp an object and hold it in part or entirely using a single hand. Although there is a wealth of behavioural data regarding grasping movements in humans and apes, there is relatively little material on macaques. To date, evidence regarding freeranging macaques is confined to observational data, while quantitative reports describe studies carried out in laboratory settings or in captivity. Here we provide an overview of recent studies

6th European Federation for Primatology Meeting
Folia Primatol 2015;86:235-386 DOI: $10.1159 / 000435825$ 
providing the kinematic descriptions of basic grip behaviour in free-ranging macaque monkeys (Macaca sylvanus and M. fascicularis). Video footage of these animals grasping objects was analysed frame-by-frame using digitalization techniques. The results revealed that the types of grips considered are each characterized by specific kinematic signatures. It was also found that hand kinematics are scaled depending on the context and the intrinsic properties of the object to be grasped. The findings outlined here regarding naturally free-ranging macaques complement previous reports and provide new insights on grip kinematics in non-human primates that may ultimately have implications in connection with evolutionary models of manual abilities. All the research complied with the European Directive 2010/63/EU.

\section{Not Just Lemurs on the Menu: Assessment of Feeding Habits and General Prey Preferences among Introduced and Endemic Predators at the Bezà Mahafaly Special Reserve, Madagascar Using Scat Sampling}

Michelle Sauther ${ }^{a}$, Michael Strinden ${ }^{a}$, Ibrahim Antho Youssouf Jacky ${ }^{b}$, Frank Cuozzo ${ }^{a, c}$, Dylan Boeken ${ }^{d}$

aDepartment of Anthropology, University of Colorado, Boulder, Colo., USA; 'bepartment of Animal Biology, University of Toliara, Toliara, Madagascar; 'Department of Anthropology, University of North Dakota, Grand Forks, N.Dak., and dDepartment of Ecology and Evolutionary Biology, University of Colorado, Boulder, Colo., USA

E-Mail: sauther@colorado.edu

There has been considerable research on understanding the dynamics of predation upon lemurs but, to date, these studies have either focused on only one predator (e.g. the fosa, Cryptoprocta ferox) or assessed predator communities in terms of temporal overlap or activity patterns using camera trap methodology. While important, these studies do not provide information on relative dietary behaviours of predators, and thus the potential impact of various predator guilds on local flora and fauna is currently missing. Using scat sampling $(\mathrm{n}=31)$ across a 10 -month period (2008-2009), this study assessed overall patterns of types of prey used by season, by month as well as prey preference by felids (Felis sp.), canids (Canis lupus familiaris), civets (Viverricula indica) and the fosa at the Bezà Mahafaly Special Reserve, Madagascar. All predators were omnivorous, using a variety of prey that included arthropods, reptiles, mammals and even plant material. Vertebrate prey was taken more during the wet as compared to the dry season. Comparisons of the two major introduced predators, felids and civets, indicate that while the two overlap in use of vertebrate prey, civets focused more on arthropods during the dry season. Identifiable remains included rats as well as lemurs, with lemur remains associated only with felids. Overall this study indicates that predators may be having a substantial effect on avian and, especially, small mammal and reptile populations as well as on lemurs, and that lemur predation must be understood in terms of overall predator patterns. Scat sampling provides a relatively easy to use and important tool to assess community prey patterns at other sites in Madagascar and elsewhere. 


\title{
Responses to Habitat Disturbance by Nocturnal Lemurs on the Masoala Peninsula
}

\author{
Rachel Sawyer $^{a}$, Zo Samuel Ella Fenosoa ${ }^{b}$, Giuseppe Donati ${ }^{a}$ \\ axford Brookes University, Oxford, UK; ' University of Antananarivo, Antananarivo, \\ Madagascar \\ E-Mail: rachelsawyer2704@hotmail.com
}

Madagascar is one of the world's 'biodiversity hotspots'. The island's plethora of endemic biodiversity is severely threatened by habitat disturbance due to tavy (slash and burn agriculture), land conversion for rice cultivation, illegal hardwood logging and bushmeat hunting. Understanding species specific responses to this habitat disturbance is crucial when designing conservation plans. We surveyed three nocturnal lemur species in four forest types of varying habitat disturbance on the Masoala Peninsula, northeastern Madagascar. We present here the first abundance and density estimates for the Endangered Avahi mooreorum and Lepilemur scottorum, and a currently undescribed Microcebus sp. Distance sampling surveys were conducted on 11 transects, covering a total of $33 \mathrm{~km}$. Overall density estimates for the study area are provided for $\mathrm{Mi}$ crocebus sp. and Avahi mooreorum using Distance 6.0. We collected data on tree height, bole height, DBH, canopy cover and tree density using point quarter sampling to characterise the four forest types (primary lowland, primary littoral, selectively logged and agricultural mosaic). Species encounter rates ranged from 1.44-2.78 ind $/ \mathrm{km}$ (Microcebus sp.), $0.22-2.89 \mathrm{ind} / \mathrm{km}$ (Avahi mooreorum) and 0-1.22 ind $/ \mathrm{km}$ (Lepilemur scottorum). Species density estimates were calculated at $232.31 \mathrm{ind} / \mathrm{km}^{2}$ (Microcebus sp.) and $121.21 \mathrm{ind} / \mathrm{km}^{2}$ (Avahi mooreorum), while no density estimate is provided for Lepilemur scottorum due to a small sample size. Microcebus sp. showed no significant effect of forest type on abundance, and its small body size, omnivorous diet and generalised locomotion appears to allow it to tolerate a variety of habitat disturbance. Both Avahi mooreorum and Lepilemur scottorum showed significant effects of forest type on their abundance. This study would suggest that the specialist locomotion and diet of Avahi mooreorum and Lepilemur scottorum make them more susceptible to the effects of habitat disturbance than $\mathrm{Mi}$ crocebus sp.

\section{Monitoring Energy Balance in Yellow Breasted Capuchins (Sapajus xanthosternos) by Measuring Total T3 in Non-Invasively Collected Faecal Samples}

Franka S. Schaebs ${ }^{a}$, Tanja E. Wolf ${ }^{b}$, Verena Behringer ${ }^{a}$, Tobias Deschner ${ }^{a}$

${ }^{a}$ Max Planck Institute for Evolutionary Anthropology, Department of Primatology,

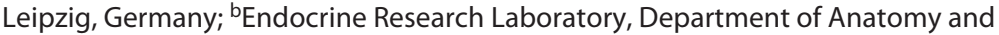
Physiology, Faculty of Veterinary Science, University of Pretoria, Onderstepoort, South Africa E-Mail: franka_schaebs@eva.mpg.de

Variation in energetic condition has a decisive impact on life history, reproductive success and, ultimately, fitness. Most existing methods to assess energy balance are relatively invasive. Only recently, the measurement of urinary c-peptide allowed for the non-invasive monitoring of energy balance in wild-living animals. Since in most species urine collection is impossible in the wild, it is important to find methods to monitor energy balance in faeces, a matrix that is much easier to collect from a variety of species. Initial studies indicate that the measurement of faecal thyroid hormone levels might allow for the monitoring of energy balance. Thyroid hormones are involved in the regulation of metabolic processes. During periods of energetic constraint, excretion is lowered leading to a reduction in metabolic activity. Here, we validated a method to measure T3, one of the two major thyroid hormones, in faecal samples of male 
yellow breasted capuchins (Sapajus xanthosternos), using a commercial total T3 (TT3) ELISA. We conducted a calorie restriction experiment on seven captive males from two social groups while assessing daily caloric intake and determining faecal TT3 levels. The chemical validation showed that the assay reliably measures TT3 levels in faecal samples of yellow breasted capuchins in terms of parallelism, extraction efficiency and recovery. Furthermore, we found daily calorie intake to be a significant predictor of TT3 levels, with TT3 being significantly lower during periods of low caloric intake. We conclude that thyroid hormone metabolites can be easily extracted and measured from faecal samples and represent a useful marker for assessing the energetic condition of yellow breasted capuchins. In general, this method might have the potential to serve as a means to monitor energy balance in species for which access to urine samples is limited.

\author{
Who Do They Look at? The Social Structure of Attention in Mandrills \\ Gabriele Schino ${ }^{a}$, Matteo Sciarretta ${ }^{b}$ \\ astituto di Scienze e Tecnologie della Cognizione, Consiglio Nazionale delle Ricerche, \\ and 'bipartimento di Fisiologia e Farmacologia 'Vittorio Erspamer', Sapienza Università di \\ Roma, Rome, Italy \\ E-Mail: g.schino@istc.cnr.it
}

Investigating who primates look at can inform us about how they perceive their social environment and what constitute the most relevant social stimuli. In this study, we conducted focal animal observations on a captive group of mandrills (Mandrillus sphinx) formed by 15 monkeys. We evaluated the factors affecting both between-individual and within-individual variation in the amount of attention paid to group mates. Neither sex nor rank affected the total amount of attention given to group mates. An analysis of within-individual variation showed that mandrills looked preferentially at high-ranking individuals and at their kin. Furthermore, after an aggressive episode the attention directed to both the aggressor and its victim increased dramatically. These results show mandrills monitor their social environment actively, thus (presumably) gaining valuable information. This research complied with the European Directive 2010/63/EU.

\author{
Retirement Programmes for Former Laboratory Chimpanzees \\ Eva Schippers ${ }^{a}$, Signe Preuschoft ${ }^{b}$, Godelieve Kranendonk ${ }^{a, c}$ \\ ${ }^{a} A A P$, Rescue Centre for Exotic Animals, Almere, The Netherlands; ${ }^{b}$ Vier Pfoten, Vienna, \\ Austria; 'Animal Ecology, Department of Biology, Utrecht University, \\ Utrecht, The Netherlands \\ E-Mail: eva.schippers@aap.nl
}

Chimpanzees have been used in biomedical research since before WWII. Vaccines and treatments for HIV/AIDS, hepatitis, malaria and other human diseases were expected to be found when using chimpanzees as 'models'. These chimpanzees were both state-owned and corporately owned by laboratories. By the 1990s, more chimpanzees lived in laboratories than were needed. Meanwhile, research on the cognitive, emotional and social nature of chimpanzees had revealed such profound similarities between humans and chimpanzees that when research on these animals was no longer necessary, euthanasia of these 'surplus' chimpanzees was judged unethical and their permanent retirement in sanctuaries was recommended (US National Research Council, 1997). A worldwide wave of retirement projects ensued. Pensions were negotiated with the chimpanzee owners: in Austria from 1999-2002, in The Netherlands from 2002-2006, in the USA from 2000-2013, in Japan from 2000-2007 and the retirement of around 50 chimpanzees 
from Gabon's CRMF is currently under negotiation. The costs of these retirement programmes are substantial, ranging from EUR 15-56 per day for one chimpanzee, with an average life expectancy of 45 years. The retirement programmes are usually run by NGOs, with the support of scientific advisory committees. The chimpanzees' biographies are usually comparable when it comes to maternal and social deprivation, stimulus deprivation, species-inappropriate keeping conditions and invasive research protocols. These factors have contributed to the development of symptoms similar to post-traumatic stress disorder and depression, impaired social competence, and the performance of abnormal behaviour. Their rehabilitation is challenging, and psychopharmaca may be necessary to use before behavioural therapy will have adequate effect. Unintentionally, at the end of their life as biomedical 'models', these chimpanzees facilitate our understanding of the evolutionary bases of psychiatric diseases.

\title{
Patterns of Migration in Male Wild Assamese Macaques
}

Oliver Schülke, Julia Ostner

Department of Behavioural Ecology, University of Göttingen, Göttingen, Germany

E-Mail: oschuel@gwdg.de

Primary and secondary dispersal are key life history events that may have pronounced effects on survival and reproduction. Migration between groups may be associated with increased predation risk if it involves longer periods of solitary ranging or it may be associated with costs accruing from active resistance to immigration by residents. In males, migration between groups may change the migrant's access to receptive females and his competitive situation. Here we quantify the timing of primary and secondary dispersal, the choice of the target group and other patterns in male migration such as parallel dispersal, dual group membership and the formation of a new group. We present data collected over the past decade on 1-4 groups of wild Assamese macaques (Macaca assamensis) in their natural habitat at Phu Khieo Wildlife Sanctuary, Thailand. These results will help to derive predictions for future studies on the adaptive value of male migration decisions.

\section{The Effects of Sociability on Independent Exploration in Wild Immature Orangutans}

\author{
Caroline Schuppli, Sofia Forss, Julia A. Kunz, Maria A. van Noordwijk, Carel P. van Schaik \\ University of Zurich, Zurich, Switzerland \\ E-Mail: caroline.schuppli@aim.uzh.ch
}

Earlier studies have shown that immature orangutans learn their feeding and nest building skills over multiple years through a combination of individual and social learning. Here we ask how these two learning mechanisms are interconnected by comparing indicators for individual and observational learning in two populations of wild orangutans that differ in their level of sociability. Individuals at Suaq Balimbing, Sumatra (Pongo abelii) spend more time in association and a higher share of their association time in close proximity $(<5 \mathrm{~m})$ to others than individuals at Tuanan, Borneo (Pongo pygmaeus). This study confirms the positive correlation between sociability and independent learning. In both populations, during larger associations immature individuals show increased levels of explorative behaviours. Furthermore, after peering (attentive close range watching over 5 seconds), immatures show significantly higher rates of explorative behaviours. Also, complex behaviours such as extractive foraging are peered at repeatedly; between peering bouts, the peering individual tries to replicate the observed behaviour. However, 
we also found that immatures at Suaq show significantly higher rates of explorative behaviours than their peers at Tuanan, even after controlling for differences in party size, which suggests a species difference. Furthermore, the Suaq population shows an overall broader cultural repertoire with a greater variety and more complex skills. Also, individuals at Suaq show increased levels of peering, even after correcting for differences in opportunities to peer. Our results strongly suggest that sociability not just facilitates the spread and maintenance of innovations in a population, but also positively affects innovation rates per se through increased exploratory tendency. Higher attentiveness in the more sociable population hints at a social learning cascade which further amplifies the positive effects of sociability on innovation.

\title{
Unveiling the Role of Social Tolerance in Play and Facial Communication in the Genus Macaca
}

Chiara Scopa ${ }^{a, b}$, Roscoe Stanyon ${ }^{c}$, Elisabetta Palagi ${ }^{b, d}$

aDipartimento di Biologia, Università di Pisa, Pisa, and ${ }^{b}$ Museo di Storia Naturale e del Territorio, Università di Pisa, Calci, 'Dipartimento di Biologia, Università di Firenze, Firenze, and IIstituto di Scienze e Tecnologie della Cognizione, CNR, Roma, Italy

E-Mail: chiara.scopa@gmail.com

Play enhances social competence and promotes bonding. In play sessions, individuals learn to communicate using signals, particularly facial expressions. The role of play signals varies according to a species social style. Macaque societies vary on a gradient ranging from intolerant (Grade 1) to tolerant (Grade 4). Tolerant macaques show less rank- and kin-constrained relationships than despotic macaques. We compared the playful signal modality and mimicry in two species of macaques placed at the opposite of this scale: Macaca fuscata (Grade 1) and Macaca tonkeana (Grade 4). We focused on facial expressions which are frequently observed during play. We considered both specific (Play Face and Full Play Face) and unspecific playful expressions (Bared-teeth, Lip-smacking and Scalp Retraction). Compared to despotic species, tolerant species show redundancy and complexity of signals (Maestripieri, 1999; Waller and Micheletta, 2013). Compared to Japanese macaques, Tonkean macaques showed a higher level of unspecific expressions during playful interactions. Considering specific playful expressions, we found that $M$. tonkeana had a higher frequency of Full Play Faces but M. fuscata expressed more Play Faces. Both specific and unspecific facial expressions were mainly performed when the animals were in direct visual contact. This result suggests that the emission of facial expressions should be intentionally used to communicate a positive playful mood. We also found that M. tonkeana individuals who spent much time playing together, also performed the most long-lasting Full Play Faces. However, this correlation was not observed in M. fuscata. Tonkean macaques engaged in longer play sessions compared to Japanese macaques. In comparison, the play interactions of Japanese macaques appear extremely fragmented suggesting a high level of competition between players.

\section{Behavioural Flexibility by Mountain Gorillas when Ranging on Community Land and Feeding on Crops}

\author{
Nicole Seiler, Martha M. Robbins
}

Max Planck Institute for Evolutionary Anthropology, Leipzig, Germany

E-Mail: nicole_seiler@eva.mpg.de

A major challenge of wildlife conservation is the growing conflict arising from interactions between humans and wildlife. Ranging outside the park and feeding on human crops by mountain gorillas (Gorilla beringei beringei) around Bwindi Impenetrable National Park, Uganda, is a 
major conservation concern. However, as competition for resources is likely to intensify, the ability of animals to exhibit behavioural flexibility is crucial for their survival in anthropogenic environments. The objective of this study was to investigate the ecological factors influencing gorilla ranging and crop feeding behaviours outside the national park. We examined the relationship between food availability in both community land and the adjacent park to the number of days gorillas ranged outside the park and consumed crops. Using Generalized Linear Mixed Models, we found that ranging outside the park and crop feeding by gorillas was positively influenced by the availability of food resources (herbaceous plants and crops) outside the park. Our results suggest that gorillas do not range outside the park and crop feed in response to forest food scarcity, but they exhibit behavioural flexibility by utilizing food resources outside the protected area. However, the density of gorillas in Bwindi is probably increasing which might push some groups to range outside the park. This increased pressure on food resources outside the park will likely augment 'human-gorilla conflicts'. This study highlights how identifying the ecological determinants of wildlife ranging and crop feeding are crucial if we hope to better understand gorilla adaptability in the face of anthropogenic habitat alteration. Our research complied with the International Primatological Society (IPS) Guidelines for the Use of Nonhuman Primates in Research.

\section{Linguistic Laws in Primate Vocal Communication}

Stuart Semple ${ }^{a}$, Ramon Ferrer-i-Cancho ${ }^{b}$, Thore Bergman ${ }^{c}$, Minna Hsu $^{d}$, Govindasamy Agoramoorthy ${ }^{e}$, Morgan Gustison ${ }^{c}$

${ }^{a}$ Centre for Research in Evolutionary and Environmental Anthropology, University of Roehampton, London, UK; ${ }^{b}$ Complexity and Quantitative Linguistics Lab, Departament de Llenguatges i Sistemes Informatics, TALP Research Center, Universitat Politecnica de Catalunya, Barcelona, Spain; 'Department of Psychology, University of Michigan,

Ann Arbor, Mich., USA; 'Department of Biological Sciences, National Sun Yat-Sen University, Kaohsiung, and e'College of Environmental and Health Sciences, Tajen University, Pingtung, Taiwan, ROC

E-Mail: s.semple@roehampton.ac.uk

Identifying fundamental principles that underpin diverse natural phenomena is a central goal of science. The existence of such principles, revealed by the occurrence of common statistical patterns, can shed light on the basic processes shaping biological systems. In recent years, exploration of the universality - beyond our own species - of the statistical laws of human language has proved a fruitful starting point for identification and investigation of these fundamental principles. In this talk, we will describe studies in which we have tested whether non-human primate vocal communication conforms to language laws. We find evidence, firstly, that the vocal behaviour of Formosan macaques (Macaca cyclopis) conforms to Zipf s law of brevity, which describes a tendency for more frequently used words (calls, in our case) to be shorter. Secondly, we find evidence that the vocal sequences of male geladas (Theropithecus gelada) follow Menzerath's law, which predicts a negative relationship between the size of the whole (e.g. a sentence, or in our case a vocal sequence), and the size of its constituents (e.g. words, or calls). We argue that these findings provide evidence that compression - the information theoretic principle of minimizing the expected length of a code - is a general principle of animal (including human) behaviour, reflecting selection for energetic efficiency. Research complied with the International Primatological Society (IPS) Guidelines for the Use of Nonhuman Primates in Research. 


\section{‘PRIMA-T' (http://primates.inecol.edu.mx): A Virtual Library of Theses on Primates}

\section{J.C. Serio-Silva}

Red de Biología y Conservación de Vertebrados, Instituto de Ecología AC, Xalapa, and Estación de Investigación Primatológica y Vida Silvestre, Balancán, Tabasco, México

E-Mail: juan.serio@inecol.mx

The number of students interested in primatology in Mexico has increased over the last few years. Unfortunately, even though these students undertake excellent research to obtain their academic degrees, such information is often available only from remote 'traditional libraries' as a printed thesis. In addition, the information in these theses is frequently not available because the authors never tried to publish the material as scientific articles, mainly because of the limitations of writing in a different language than Spanish. Therefore, a virtual library of theses about primates (Prima-T: http://primates.inecol.edu.mx) has been created that enables all theses on Mexican primates, or on exotic primates that have been written in Mexico, to be stored in a digital format. Since February 2014, when the virtual library was created, until January 2015, a total of 120 theses written in Mexico have been made available and the website has been visited by nearly 4,100 people. Visitors can get free access and register in a simple way and can almost immediately get the file of the thesis that interests them. Although all dissertations were made available in Mexico, there has been considerable interest in the library; we have documented visits from more than 43 countries and there have been nearly 300 registered users making queries. Finally, it has always been said that theses are 'grey documents' because of their limited distribution. Our intention is that, in addition to the many scientific publications that are produced, theses can be accessed as these always contain many more details of the investigations carried out and they also serve as a motivation for more students in Mexico and the world. Our research complied with the European Directive 2010/63/EU and/or the International Primatological Society (IPS) Guidelines for the Use of Nonhuman Primates in Research.

\section{Identity and Conservation Models in Balancán, Mexico: Key to Saving the Critically Endangered Mexican Black Howler Monkey}

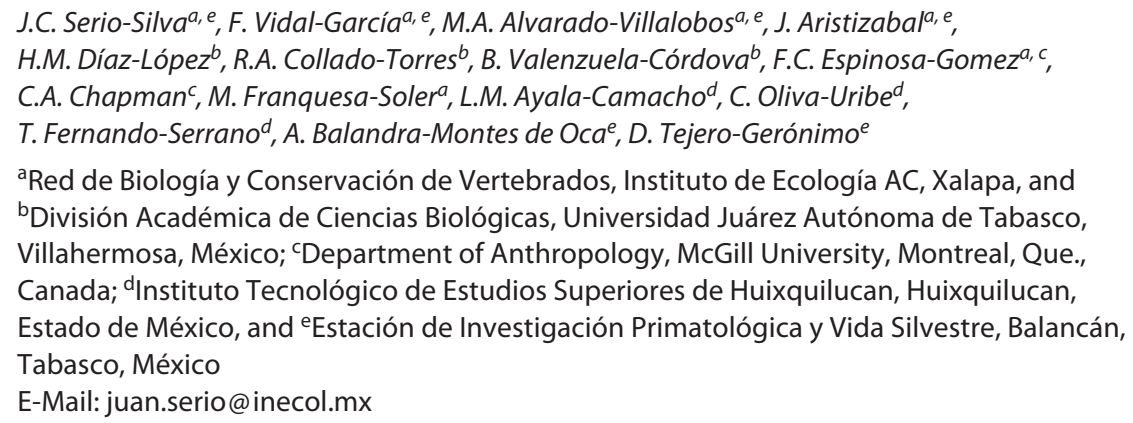

After many years of identifying the problematic situation of wild monkeys and their habitat, we have seen that we can have a positive effect on conservation only if people are involved with elements of the environment with which they can identify and feel represented. To do this, our research team developed an identity and conservation model for the care of the black howler monkey (Alouatta pigra) and its habitat in the Municipality of Balancán, Tabasco, Mexico. With our support, municipal authorities agreed to develop a formal declaration, where this town is now identified as the 'Sanctuary of the Sacred Black Howler Monkey'. As part of the celebration for this declaration we have held two great festivals called the 'First (2013) and Second (2014) Internation- 
al Black Howler Monkey Week'. The main activities that took place were: lectures and primate related workshops, guided tours to field sites, habitat restoring activities, exhibitions of primate handicrafts made by people from local communities and cultural activities (dancing, singing, poetry, theatre, a mural was painted, movies). These were all offered to the people attending $(>4,000$ during each Week) who were learning about their monkeys and the importance of preserving tropical rain forest. One urgent activity is promoting more environmental education in places where scientific research is conducted. We cannot be only spectators, instead we must act, researching, protecting and restoring. Only then will our primate diversity and communities be able to live in harmony. Our research complied with the European Directive 2010/63/EU and/or the International Primatological Society (IPS) Guidelines for the Use of Nonhuman Primates in Research.

\section{Description of New Hominoid Specimens from the Late Miocene Locality of Çorakyerler, Turkey}

Ayla Sevim Erol ${ }^{a}$, Alper Yener Yavuz ${ }^{b}$, Erhan Tarhan ${ }^{c}$

${ }^{a}$ Department of Anthropology, Faculty of Languages, History and Geography, Ankara University, Ankara, ${ }^{b}$ Department of Anthropology, Faculty of Arts and Science, Mehmet Akif Ersoy University, Burdur, and 'Department of Anthropology, Faculty of Arts and Science, Mustafa Kemal University, Serinyol, Turkey

E-Mail: ayla_sevim@yahoo.com

The Çorakyerler vertebrate fossil locality, dating to the late Miocene, is located along a road in Yapraklı district in Çankırı province in the Central Anatolia Region of Turkey. The altitude of the site is about $745 \mathrm{~m}$. Excavations have been held systematically in this region since 2001. The Çorakyerler vertebrate fossil locality is between MN11-12 zones (about 8-7 million years old) according to faunal comparisons and magnetostratigraphic dating. Çankırı-Çorum Basin, on which the fossil site is located, emerged at low tide of the Tetis Sea. Çorakyerler, where so many different species, from members of the Giraffidae to Rhinocerotidae, from Carnivora to Hominoidea, lived, is a significant site because of its faunal diversity and the quality of the findings. Fossils of animals which are now extinct in Çankırı, but were alive in Çorakyerler in the late Miocene, were found in the excavations. In the excavations held since 2001, hominoid teeth, mandibular and maxilla fragments from five different individuals have been found. The palate, which was discovered first, belongs to a male. The second discovery, an upper third molar, belongs to an adult individual. The third discovery was a mandibular corpus that is thought to belong to a juvenile male. The fourth one was a mandibular fragment belonging to a female. The fifth was a central incisor that also belongs to an adult individual. In addition to the isolated tooth, mandible pieces and maxilla fragments, in 2010 a face (a rare find for hominoids in general) was found in this locality.

\section{All in the Family: Cooperation and the Origin of Female Kin Bonding and Its Impact on Reproductive Skew}

Susanne Shultz

University of Manchester, Manchester, UK

E-Mail: Susanne.shultz@manchester.ac.uk

The prevalence of kin selection as a framework for understanding the evolution of cooperation has been totally pervasive in the field of evolutionary ecology. High levels of within group relatedness, particularly as a consequence of social monogamy or delayed dispersal, lead to indirect fitness benefits of cooperating with neighbours. Here we demonstrate that neither 
social monogamy nor kin groups explain the evolution of cooperation in primates. Rather cooperation emerges as a consequence of direct benefits. Moreover, both social monogamy and kin structuring emerged, not before, but after cooperative sociality was established. Thus kin bonding in primates is a secondary transition following the emergence of cooperation rather than facilitating its evolution. Moreover, female bonding, coupled with dominance hierarchies, can result in high levels of within group competition. We argue that in such cases, kin bonding can result in high levels of reproductive inequality. Thus, kin structure, and particularly female bonding, has more nuanced relationships with reproductive success and fitness than the textbook story.

\section{What Influences Seasonal Body Weight Variation in Primates? A Comparative Study of Life History Profiles and Seasonality, Using Data Collected by Duke Lemur Center on Twenty-Seven Captive Strepsirrhines}

Karolina Simanaityte, Caroline Ross

University of Roehampton, London, UK

E-Mail: simanaik1@ roehampton.ac.uk

Long-term field studies of primates are particularly important. They enrich our understanding of population dynamics, behaviours and other aspects of primate life histories. However, as primates tend to live longer than other animals of similar body size, they are often difficult to follow for decades. Since 1966, Duke Lemur Center (DLC) has collected comprehensive information about its resident strepsirrhines. Nearly fifty years' worth of life-history data, gathered on twenty-seven taxa, and more than three thousand individuals, are now freely available online. Preliminary reports by DLC discovered that the most radical body weight changes occurred in small, heterothermic lemurs (Cheirogaleus medius and Microcebus murinus). The average winter weights were significantly higher than summer weights. Previous studies propose that seasonality often occurs in resource challenged animal groups, particularly during resource poor (dry) seasons. As captive strepsirrhines at DLC are not food deprived, the aim of this project is to use the data published by DLC to (1) explore what variables may influence seasonality and (2) how seasonality varies between species. We hypothesise that (1) all species covered in this report will exhibit seasonality and that (2) variation in the degree of seasonality may be linked to a variety of environmental variables. Results on the relationship between seasonality, other life history variables and species' ecology will be presented. This study complies with the European Directive 2010/63/EU and the International Primatological Society (IPS) Guidelines for the Use of Nonhuman Primates in Research.

\section{Do Chimpanzees Anticipate an Object's Weight? A Field Experiment on the Kinematics of Hammer-Lifting Movements in the Nut-Cracking Taï Chimpanzees}

Giulia Sirianni, Roman Wittig, Christophe Boesch

Department of Primatology, Max Planck Institute for Evolutionary Anthropology, Leipzig, Germany

E-Mail: giulia_sirianni@eva.mpg.de

Dexterous handling of objects is essential for many animals, particularly those relying on tools for foraging. Weight is a major determinant of interactions with objects, and being able to anticipate an object's weight is beneficial in dealing with the physical world (e.g. enabling one to 
apply the correct amount of force to act on objects). However, whether animals anticipate the weight of novel objects is still unknown. In this study we ran a field experiment to test whether wild chimpanzees (Pan troglodytes) in the Taï forest (Côte d'Ivoire), which habitually crack nuts using hammers and anvils, anticipate the weight of hammers by displaying an overshoot effect when lifting hammers that look identical but are of different weight. Overshoot effect is observed when a mismatch between expected and actual object weight causes an object that is lighter than expected to be lifted with a higher acceleration than a similar, heavier, object. We set up 8 lab-sites in the Taï forest, each site supplied with a camera trap, nuts, and one hammer. Two kinds of hammers were used: natural and modified. They were of identical size and material, but were different weights, with the modified hammer being lighter (core material removed) than the natural one ( 1.5 vs. $2.5 \mathrm{~kg}$ ). We used calibrated video recordings to estimate the acceleration for the first lift of the hammer in each nut-cracking episode and compared it between the two hammers. We predicted that, if chimpanzees anticipated the weight of the hammer based on its external appearance, they would show an overshoot effect by lifting the lighter (modified) hammer with a higher initial acceleration than the natural hammer. We collected 74 trials by 14 individuals. Preliminary results from a GLMM showed the expected difference in acceleration between hammers. However, further analyses are in progress to eliminate alternative interpretations, and these results will be presented. The study complied with the European Directive 2010/63/EU.

\title{
Long-Term versus Short-Term Consistency of Social Personality Traits and Salivary Cortisol Levels in Common Marmosets
}

\author{
Vedrana Šlipogor ${ }^{a}$, Jorg J.M. Massen ${ }^{a}$, Eva Millesi ${ }^{b}$, Thomas Bugnyar ${ }^{a}$ \\ Departments of a Cognitive Biology and ${ }^{\mathrm{b}}$ Behavioural Biology, University of Vienna, \\ Vienna, Austria \\ E-Mail: vedrana.slipogor@univie.ac.at
}

Animal personality stands for a correlated suite of non-social (boldness-shyness, exploration-avoidance and activity) and social (aggressiveness and sociability) behavioural traits, consistent across time and/or contexts. Although studies of personality rely heavily on repeatability of data, both short-term (days/weeks) and long-term (months/years) repeatability have rarely been tested. Physiological mechanisms that may provide a proximate explanation for the maintenance of a consistent inter-individual variation are also relatively unexplored, especially in combination with behavioural data. In this study we measured both behavioural and physiological responses of common marmosets (Callithrix jacchus) in two behavioural tests (Mirror and Video Test), repeated after a short seventeen day break and a long one year break. In the Mirror Test, we presented our subjects with a mirror, and in the Video Test, we replaced the mirror with a computer screen on which a video recording of an unfamiliar same-sex individual was presented. In addition, saliva samples were taken immediately before and ten minutes after the tests, and from these we deduced corresponding delta values. In both tests, we predicted that seeing an 'unfamiliar conspecific' would evoke social (affiliative or agonistic) and physiological (increase or decrease in salivary cortisol levels) responses in this highly territorial species, and that the degree of this response should vary consistently between individuals. We will discuss whether both behavioural and endocrine responses are consistent in both the short- and long-term, and whether inter-individual differences in cortisol levels are correlated to social personality traits, i.e. whether more sociable individuals show a greater increase or decrease in salivary cortisol levels after seeing an unfamiliar conspecific than less social individuals. This study complied with the International Primatological Society (IPS) Guidelines for the Use of Nonhuman Primates in Research. 


\section{Seasonal Variability in Association Patterns in a Wild Group of Spider-Monkeys (Ateles geoffroyi)}

Sandra E. Smith Aguilar, Gabriel Ramos-Fernández

CIIDIR Unidad Oaxaca, Instituto Politécnico Nacional, Oaxaca, Mexico

E-Mail: galadrielent@yahoo.com

The constant change in subgroup size and composition observed in species with high fission-fusion dynamics (HFFD) may result in distinct individual patterns of aggregation and space-use. Evidence suggests that shifts in food availability and distribution are determinants of the overall grouping patterns. However, little is known about the constraints that HFFD pose on social relationships and, in turn, how these influence space use. This problem can be approached by analysing the relationships between association and space use patterns and how these change over time (socio-spatial patterns). Socio-spatial models predict unstable associations and a loose spatial structure for egalitarian (vs. despotic) societies like those found in spider monkeys. We present results of permutation-based analyses to detect associations in a wild group of spider monkeys (Ateles geoffroyi) using data collected between January 2013 and September 2014 at the Otoch Ma'ax Yetel Kooh protected area in Mexico. Associations were based on observations of two individuals in the same subgroup in 4630 instant scans collected every 20 min throughout 326 days during group follows of 4-8 daily hours. Analysis of the whole period indicated the presence of non-random associations (preferred and avoided). Further results of seasonal patterns of association and their variation indicated that preferential associations between some dyads were only apparent during a particular season or period. These results will be discussed in light of a significant decrease in individual core area size and increase in mean subgroup size in wet versus dry seasons. These conditions involve a shift in the overall social context experienced by individuals and the potential increase of a 'third party effect' owing to a higher probability of encounter and the increased presence of members of the opposite sex in subgroups. This project complied with International Primatological Society Guidelines for the Use of Nonhuman Primates.

\section{Modeling Population Viability of Local Javan Gibbon (Hylobates moloch) Populations}

Jaima Smith, Vincent Nijman

Oxford Brookes University, Oxford, UK

E-Mail: jaimahillary@gmail.com

Population viability analysis (PVA) is a predictive procedure that uses a combination of different modelling approaches to estimate species vulnerability to extinction. Two decades ago, a PVA was run for the endangered Javan gibbon (Hylobates moloch), however, the resulting report suffered from a lack of information at the population level and a lack of data on variability between sites. With the aim of addressing the weakness of the first PVA, by using the stochastic modelling software VORTEX, we have now assessed the status of Javan gibbons in three areas (Ujung Kulon, Halimun and Dieng) which hold over half of the remaining total number of gibbons on Java. Ujung Kulon and Halimun are long-time protected areas, whereas Dieng remains largely unprotected. We used species-specific and, where possible, site-specific, life-history parameters. For each area, we calculated the probability to extinction over a 50 -year time period. We ran the model twice, (1) assuming each area offered high levels of connectivity for the gibbons, and (2) assuming a more fragmented distribution. We tested the sensitivity of the final predicted population size to $20 \%$ variation including the likelihood of inaccurate parameter estimates. Our modelling suggests that none of the three populations is in immediate danger of ex- 
tinction, but all three have suffered significant population declines. We investigated different management strategies that may help forestall population extirpation at each site, such as: reforestation, connecting corridors and hunting reduction by humans. We concluded that specific actions should be implemented in the short-term, particularly at lower elevations where Javan gibbons reach their highest densities.

\section{Population Viability Analysis for Cercocebus atys lunulatus}

Sònia Sánchez-López ${ }^{a}$, Anaïs Avilés de Diego ${ }^{b}$, Joaquim Josep Veà-Barób, Arturo González Zamora ${ }^{a}$

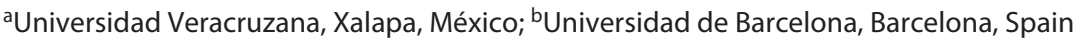

E-Mail: sosanchez@uv.mx

Habitat loss and fragmentation are deterministic processes that lead to the extinction of species as they reduce the size of populations, making them more sensitive to environmental, demographic and genetic stochastic processes. The aim of this study was to evaluate the effects of habitat fragmentation on wild populations of the white-naped mangabey (Cercocebus atys lunulatus) inhabiting forest fragments in Ghana and Ivory Coast to establish criteria for future conservation strategies. We reviewed published articles, book chapters and data on wild populations of mangabeys, looking at: records by habitat type, species biology and potential threats. With all this information we conducted a Population Viability Analysis (PVA) using the VORTEX program. We simulated scenarios with deterministic and stochastic effects, a combination of both, on large and small populations living in continuous and fragmented habitat. Likewise, we evaluated probability of persistence, population growth, number of individuals and genetic diversity of C. a. lunulatus to 100 years. The results show that populations of C. a. lunulatus need at least an initial size of 5,000 individuals to ensure their persistence in the near future, along with the establishment of a continuous population. Similarly, conservation and survival of the species in both countries can be achieved only if habitat degradation is quickly reduced. If the annual habitat degradation exceeds $4 \%$, populations of white-naped mangabey in Ghana and Ivory Coast are destined to become extinct within 30 years, so it is urgent to develop effective conservation strategies for this species. Mangabeys in West Africa need greater conservation attention and the present Vortex simulation may aid conservation of Cercocebus atys lunulatus. This research complied with the European Directive 2010/63/EU and/or the International Primatological Society (IPS) Guidelines for the Use of Nonhuman Primates in Research.

\section{The Role of Social Styles in Grooming Patterns and Stress Alleviation \\ Ruth S. Sonnweber ${ }^{a}$, Andrea Ravignani ${ }^{a}, b$, Nina Stobbe ${ }^{a}$, Gisela Schiestla , Bernard Wallner ${ }^{a}$, W. Tecumseh Fitch ${ }^{a}$ \\ aUniversity of Vienna, Vienna, Austria; 'bniversity of Edinburgh, Edinburgh, UK E-Mail: ruth-sophie.sonnweber@univie.ac.at}

Group life is associated with many challenges and the potential for conflict between individuals. To deal with these potential stressors, individuals can flexibly adapt their social behaviour to social and environmental conditions, which allows for mitigation of glucocorticoid (GC) levels. Thus social behaviour can serve as a physiological stress coping mechanism. Amongst primates, the predominant social behaviour is grooming, and, in particular, grooming others has been found

6th European Federation for Primatology Meeting
Folia Primatol 2015;86:235-386 DOI: $10.1159 / 000435825$ 
to be effective in terms of GC alleviation. However grooming is a time-consuming activity and access to grooming partners may be confined, potentially leading to different grooming patterns. Robert Seyfarth proposed that attraction to kin and high-ranking individuals, as well as competition over access to preferred grooming partners, would impact grooming distribution across social partners. Studies investigating grooming patterns in despotic primate species revealed that individuals of all rank groups show lowest GC levels when focusing their grooming activity on only a few preferred partners within their social net. Here we present data on a tolerant primate species, the Barbary macaque (Macaca sylvanus), showing a gradual connection between rank-dependent grooming patterns and GC mitigation. While higher-ranking females express lowest GC levels when concentrating their grooming on selected partners, lower-ranking females show lowest GC levels when dispersing grooming evenly across all social partners. We argue that the relaxed social style of this species comes with increased behavioural flexibility that allows females of all rank groups to optimally adapt their grooming behaviour to the ever changing challenges within a social group. The research was in accordance with the European Directive 2010/63/EU and the International Primatological Society (IPS) Guidelines for the Use of Nonhuman Primates in Research.

\title{
Testing Line Transect Surveys for Fast Moving Primates with High Levels of Fission-Fusion Dynamics
}

\author{
D. Spaan ${ }^{a, b}$, G. Ramos-Fernández ${ }^{b, c}$, C.M. Schaffner ${ }^{a, b}$, B. Pinacho-Guendulain ${ }^{c}$, F. Aurelia, $b$

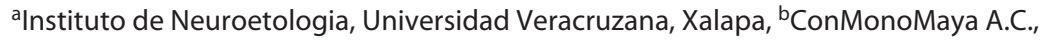 \\ Chemax, and ${ }^{\mathrm{C} C e n t r o ~ I n t e r d i s c i p l i n a r i o ~ d e ~ I n v e s t i g a c i o ́ n ~ p a r a ~ e l ~ D e s a r r o l l o ~ I n t e g r a l ~}$ \\ Regional, Unidad Oaxaca (CIIDIR-OAX), Instituto Politécnico Nacional, Oaxaca, Mexico \\ E-Mail: denisespaan@ hotmail.com
}

Spider monkeys (Ateles spp.) are large-bodied Neotropical primates threatened across their range largely due to habitat loss. Their fast movement and high degree of fission-fusion dynamics make them notoriously difficult to survey. The aim of our study was to test line transect methodology on a community of individually recognized spider monkeys (A. geoffroyi) in Punta Laguna, Yucatan, Mexico. We performed 102 walks on two transects (919 and $921 \mathrm{~m}$ ) from October 2014 to January 2015. Perpendicular distance was calculated from the transect to all individuals and to the centre of all subgroups encountered. We calculated the encounter rate (number of monkeys or subgroups / km surveyed) per walk. We ran general linear models to determine the effect of the number of observers (1,2 or 3), transect (straight or curved) and time of the day in which the survey was carried out (06:00-10:00, 10:00-14:00 or 14:00-18:00) on individual monkey encounter rate and subgroup encounter rate, while controlling for performing multiple transect walks in the same day and month. Using 'Distance' software we found that population density was possibly overestimated when using distance from the subgroup centre as it was higher than when using distance from individuals. Time of transect walk affected both individual and group encounter rates, with lower encounter rates in the middle of the day than in early morning and late afternoon. This is likely due to the lower activity level of spider monkeys in the middle of the day, making it more difficult to see them. Although transects done by one observer had on average fewer encounters than those done by 2 or 3 observers, there was no significant effect of the number of observers on encounter rate. Furthermore, we found no difference in encounter rate between the straight and curved transect. We therefore recommend that line transect surveys on spider monkeys be conducted with a minimum of 2 observers in the morning or afternoon and that the perpendicular distance to each individual encountered should be used to calculate population density. 


\title{
Consumption of Crops by Capuchin Monkeys Living in an Anthropogenic Habitat: Combining Quantitative Evaluation of Crop Loss with Farmers' Perceptions
}

\author{
Noemi Spagnolettia, ${ }^{a}$, Patricia Izar $^{a}$ \\ aUniversity of São Paulo, São Paulo, Brazil; blstituto di Scienze e Tecnologie della \\ Cognizione - CNR, Rome, Italy \\ E-Mail: noemi.spagnoletti@gmail.com
}

As proximity to cultivated areas grows, wildlife is increasingly exploiting anthropogenic foods, resulting in economic loss to farmers. Among Neotropical primates, capuchin monkeys (genera Sapajus and Cebus) stand out as frequent crop foragers. In many regions where primates interact with humans, negative perceptions generated through negative primate behaviour can threaten their conservation, especially when using economically important resources. At the same time, the rapid expansion of human activities is a major cause of deforestation and habitat fragmentation affecting primate behaviour and their ability to persist in these areas. Thus, who is the invader? Using cross-disciplinary methods, we evaluated the perceptions of farmers sharing space with S. libidinosus in Piauí, Northeastern Brazil using semi-structured interviews, and quantified the consumption of maize (a key local crop) through direct observations of three groups of capuchin monkeys. In this region, agricultural areas form part of the capuchin's home range, with native forests degraded and fragmented. Local people mostly practice subsistence agriculture. Of all crops grown in the region, farmers reported that capuchins mostly fed on maize, with individual farmers rating damage levels from 'zero' to 'very high'. Although farmers reported that capuchin monkeys are the major causes of crop damage, birds account for over a third of the total loss. The exploitation of human food sources by capuchins seems to reflect their opportunistic and flexible behaviour. Farmers must take account of this flexibility and adopt strategies to reduce crop damage, such as planting further from forest edges and water sources used by monkeys during daily activities. Research approved by the Ethical Committee of the Institute of Psychology, São Paolo University.

\section{Colour Vision in a Nocturnal Species: The Grey Mouse Lemur (Microcebus murinus)}

Caterina Spiezio $^{a}$, Barbara Regaiollia ${ }^{\text {, Camilla Cenni }}{ }^{b}$, Maria Vallisnerib

aResearch and Conservation Department - Parco Natura Viva, Bussolengo, and buniversity of Bologna, Bologna, Italy

E-Mail: spiezio@parconaturaviva.it

Two main hypotheses in the literature aim at explaining the evolution of trichromatic colour vision in human and non-human primates: the foraging hypothesis suggests a selective pressure to detect ripe fruit and young red leaves whereas the social-sexual communication hypothesis highlights that many trichromatic primates use colour signals during intra-specific communication. The aim of this study was to investigate whether and how the grey mouse lemur (Microcebus murinus), despite its dichromatic colour vision, is able to discern green and red during foraging. Seven grey mouse lemurs, 3 males and 4 females, housed in two different enclosures at Parco Natura Viva, Bussolengo (VR) were observed. Subjects were tested in their social context, using non-invasive techniques, complying with the European Directive 2010/63/EU Guidelines. Grey mouse lemurs were provided with coloured enrichment devices randomly given in different combination: white/green, green/red and red/white. For each enclosure, one daily 30 -min session (30 sessions in total) was carried out during the interaction with the enrichment devices. An all occurrences sampling method was used to collect data about the interaction with the devices. Results for male lemurs revealed a significant preference for the white cone when a white-green 
combination was provided and for the red cone in the presence of a green-red combination. Therefore, males seem to be able to distinguish between white, red and green even though they have dichromatic colour vision. Since males and females were housed in separate enclosures with different moonlight conditions, results suggest that the two groups might rely on different characteristics of the colour of an object, such as contrast or brightness. In conclusion, during the evolution of trichromatic colour vision, dichromy seems to be a profitable step in food selection, supporting the foraging hypothesis as a selective pressure for trichromy.

\title{
Determinants of Long Call Activity in Bornean Orangutans (Pongo pygmaeus wurmbii)
}

\author{
B. Spillmann ${ }^{a}$, E.P. Willems ${ }^{a}$, Maria A. van Noordwijk ${ }^{a}$, T.M. Setia ${ }^{b}$, C.P. van Schaik ${ }^{a}$ \\ aUniversity of Zurich, Zurich, Switzerland; bUniversitas Nasional, Jakarta, Indonesia \\ E-Mail: spillbri@gmx.ch
}

Long call activity of flanged male orangutans varies over time. The aim of this study is to explain these fluctuations, by examining social and ecological correlates. To date it has been impossible to answer this question, due to the orangutan's semi-solitary social organization and the resulting wide dispersion of individuals. Thus individual focal follows give an incomplete view of the males' communication system over time and space. We installed 20 microphones in an array at Tuanan field site in Central Kalimantan, Indonesia, to continuously record long call occurrences in a 900 ha area over a period of one year. We extracted 8,000 long calls from these recordings, which were timed, localized and assigned to individuals using protocols that have been independently validated. We used the long-term data on phenology and female focal follows to estimate the independent ecological and socio-sexual variables. This allows us for the first time to answer two important questions on orangutan social behaviour with a full dataset on male vocal activity. We first addressed whether long call activity correlates linearly with the number of flanged males present in the grid at a certain time, or whether this relationship is steeper (exponential). The latter would imply that males provoke other males into making long calls, perhaps in order to (re)establish dominance relationships among them. The second question is what determines the presence of males in the grid. One hypothesis is that this depends on the number of sexually active females in the grid. Flanged males might gather at shorter distances around such a female and this accumulation might lead to vocal competition over access to this female. Another, not incompatible hypothesis is that male presence and calling activity depends on food abundance, consistent with the assumption that long call production is energetically costly. Preliminary data suggest that the social factors are more important than the ecological one.

\section{The EMO-Model: An Agent-Based Model of Emotional Bookkeeping}

Elisabeth H.M. Sterck ${ }^{a}, b$, Ellen Evers ${ }^{a}$, Han de Vries $^{a}$, Berry M. Spruijt ${ }^{a}$

aUtrecht University, Utrecht, and biomedical Primate Research Centre,

Rijswijk, The Netherlands

E-Mail: e.h.m.sterck@uu.nl

Primates typically show individual-specific bonds, i.e. bonds with particular individuals from their group. Bonds result from repeated affiliative interactions between these group members, yet several processes with an increase in cognitive demands may drive this: (1) agonismbased spatial patterns; (2) symmetry-based reciprocity; or (3) emotional bookkeeping. It is debated which of these processes primates actually employ to maintain bonds and this is difficult to assess in real primates. Agent-based models, employing interacting agents with specific behav- 
iour rules, are an ideal way to investigate the social consequences of such processes. We studied patterns in bonds in the agent-based EMO-model, where the nature of encounters affects the emotional state of an individual and their attitude to interaction partners, representing a model of emotional bookkeeping. This was compared with a model of a symmetry-based situation, where dominance determines the attitude to interaction patterns, and a model of agonism-based spatial patterns. Reciprocity of affiliation is found for both the symmetry-based reciprocity and the emotional bookkeeping model. Individual-specific bonds are found in only the emotional bookkeeping model. These results are consistent with the proposition that primates employ emotional bookkeeping to maintain their individual-specific bonds.

\section{Capuchins' Behavioural and Endocrine Response to Conspecifics' Actions}

Martina Stocker ${ }^{a}$, Eoin O'Sullivan ${ }^{b}$, Ruth S. Sonnweber ${ }^{a}$, Christine A. Caldwell ${ }^{b}$

aUniversity of Vienna, Vienna, Austria; bUniversity of Stirling, Stirling, UK

E-Mail: martina.stocker@univie.ac.at

For group-living animals it is essential to understand and react to other individuals' behaviour and actions. It is necessary for a member of a social group to not only recognize and respond to its own bonding partners, but also to keep track and respond to third-party relationships, conflicts or rank relations. Noticing and understanding structural (e.g., rank reversals) and qualitative (e.g., alliances, kin relations) conditions and changes within the group allows an individual to adapt its behaviour accordingly. Social factors can act as stressors or influence group members in a positive way, which should lead to different responses. We expect that reactions to sociopositive or socio-negative situations be reflected in behavioural and/or physiological changes. Increased release of stress hormones or enhanced stress-related behaviours (e.g. scratching) in response to a threat or other stressful situations are adaptive mechanisms, which allow an individual to cope with challenges. With this study we attempt to elucidate the effects of socially valenced scenarios on an individual's behaviour and stress physiology. Additionally, we aim to identify different stress coping mechanisms among the individuals, such as the display of certain behaviours that alleviate physiological stress. Therefore, we presented capuchin monkeys (Sapajus sp.) with four conditions (video stimuli): showing a group member in (1) a socio-positive, (2) a neutral, (3) a socio-negative situation and (4) a non-social control. We recorded the subjects' behavioural reactions to the videos and monitored changes in salivary cortisol to measure their physiological stress responses. Preliminary results indicate that the different social and non-social conditions impact the monkeys' attention and that individuals within the study population exhibit different behavioural and physiological coping mechanisms. The study complied with the IPS Guidelines for the Use of Nonhuman Primates in Research.

\section{Behavioural, Biological, and Epigenetic Consequences of Early Social Experience in Rhesus Monkeys}

Stephen J. Suomi

Eunice Kennedy Shriver National Institute of Child Health and Human Development, National Institutes of Health, Poolesville, Md., USA

E-Mail: suomis@mail.nih.gov

It is now well-established that the types of early social attachment relationships that rhesus monkey (Macaca mulatta) infants form with their caregivers can have dramatic behavioural, biological and epigenetic consequences throughout development and beyond. Recent research

6th European Federation for Primatology Meeting
Folia Primatol 2015;86:235-386 DOI: $10.1159 / 000435825$ 
has focused instead on the consequences of being raised by mothers who differ in their social dominance status. There are major differences in both social opportunities on a daily basis and long-term physical and psychological health outcomes between offspring of high- versus lowranking mothers, and it appears that relative social dominance status is generally transmitted from mothers to their female offspring, i.e., high-ranking mothers typically rear daughters who themselves are high-ranking, at least initially, and low-ranking mothers usually have daughters who turn out to be low-ranking themselves. Very recent data suggest that such cross-generational transmission of relative dominance status may be in part epigenetically mediated through the placenta, i.e., there may be a biological mechanism through which specific social characteristics of certain individuals can be transmitted across generations.

\title{
Urinary C-Peptide Levels in Male Bonobos (Pan paniscus) Are Related to Gregariousness and Rank but Not to Mate Competition
}

Martin Surbeck, Verena Behringer, Tobias Deschner, Gottfried Hohman

Max Planck Institute for Evolutionary Anthropology, Leipzig, Germany

E-Mail: gritschubert77@gmail.com

Within- and between-species variation in male mating strategies has been attributed to a multitude of factors including male competitive ability and the distribution of fertile females across space and time. Differences in energy balance across and within males allow for the identification of some of the trade-offs associated with certain social and mating strategies. Bonobos (Pan paniscus) live in groups with a high degree of fission-fusion dynamics with co-dominance between the sexes and linear dominance relationships among males. Furthermore, males compete over females which are aseasonal breeders and exhibit sexual swellings over extended time periods. In this study we use urinary C-peptide (UCP) levels in male bonobos, obtained from 260 urine samples from a wild bonobo community, to quantify male energy balance during mate competition and levels of gregariousness in the species. Although high-ranking males are more aggressive, spend more time in proximity to maximally tumescent females and have higher mating frequencies, we found no indication that mate guarding or mate competition affected male energy balance. Our results showed a positive correlation between monthly mean UCP levels and mean party size. When travelling in large parties, high-ranking males had higher UCP levels than low-ranking males. These results support the hypothesis that the patterns of fission-fusion dynamics in bonobos are either linked to energy availability in the environment or to the energetic costs of foraging. The finding of a rank-bias in UCP levels in larger parties could also reflect an increase in contest competition among males over access to food.

\section{New Observations in Great Ape Conservation Research in the Dja Landscape, Cameroon}

\author{
Nikki Tagga ${ }^{a}$ Jacob Willie ${ }^{a, b}$, Donald Mbohlia \\ aProjet Grands Singes (PGS), Centre for Research and Conservation (CRC), Royal Zoological \\ Society of Antwerp, Antwerp, and ${ }^{\text {b}}$ Terrestrial Ecology Unit, Ghent University, \\ Ghent, Belgium \\ E-Mail: Nikki.tagg@ @mda.org
}

The majority of wild chimpanzee and gorilla populations in Central Africa today exist outside protected areas, where monitoring and research initiatives are critical to guiding conservation efforts and providing direct protection of vulnerable populations. The long-running, great ape con- 
servation research programme of the Centre for Research and Conservation in the Dja landscape, Cameroon has contributed to these goals since 2001 and continues to apply an integrative, multidisciplinary approach to great ape conservation in the area, while incorporating a close collaboration with and inclusion of local communities. Over the years, studies at the research site La Belgique have revealed a number of interesting socio-ecological observations of central chimpanzees (Pan troglodytes troglodytes) and western lowland gorillas (Gorilla gorilla gorilla): chimpanzees sleep in ground nests, gorillas exhibit patterns of fission-fusion, and both ape species partake in high rates of insectivory; all of which are being further investigated. Newer observations further confirm the importance of continued research in the site: the site has relevance to the study of certain great ape diseases and extensive faecal sampling is taking place to investigate prevalence, pathogenicity and implications for survival. Regular surveys over the years are beginning to reveal trends in human use of forest resources, and are helping to shape and focus the project's actions for the better acquisition of its conservation goals. Crucially, the sensitization and deterrent effect offered by the presence of the research programme itself has been proven to have a positive influence on the abundance of great apes in the area; the exact mechanism of this effect is being teased out. This talk will present the newest results of the project's most recent and important scientific research.

\section{Social Development of Juvenile Orang-Utans (Pongo pygmaeus): Play Behaviour and Interactions in a Rehabilitation Centre in West Kalimantan \\ Madeline Thalera, Susan M. Cheyne ${ }^{b}$ \\ axford Brookes University, Oxford, UK; ${ }^{\mathrm{b}}$ Orangutan Tropical Peatland Project, Palangka Raya, Indonesia \\ E-Mail: maddy.thaler-2014@brookes.ac.uk}

The role of play in mammalian development is still a debated topic. Play is often used as an indicator of adequate welfare for captive primates. However, the role it plays in development is not fully determined. Play is often described as a 'luxury' behaviour, observed when costs are low because all proximate needs are met or as a suite of behaviours critical for learning socially appropriate behaviours specific to living in large social groups. However, orang-utans (Pongo spp.) are described as semi-solitary primates that have fewer opportunities to play socially with conspecifics. Conversely, orang-utans housed together tend to play more than their wild counterparts due to increased opportunity to do so and decreased levels of food competition and time spent travelling. This discrepancy raises questions about the role of social play in orang-utan development as well as about how increased play opportunity in captivity impacts the development of orang-utans slated for reintroduction. We observed 12 juvenile Bornean orang-utans (Pongo pygmaeus), using continuous focal sampling, to record variations in types and frequencies of play across age-sex classes. Individuals were housed at International Animal Rescue's (IAR) orang-utan rehabilitation centre in Ketapang, Indonesia. Results are expected to be consistent with previous studies on play in primates that show a negative correlation with age and play frequency. Temperament is expected to correlate with frequency of play and abnormal behaviours, where individuals described as shy play less and express stereotypies more, while individuals described as gregarious play more and express stereotypies less. This is consistent with previous conclusions about play as an indicator of adequate welfare, but also stresses the importance of considering temperament when planning captive housing for primates. All research was noninvasive and adhered to the IPS Ethical Guidelines for Use of Non-Human Primates in Research. 


\title{
Male Sexual Coercion in Tonkean Macaques: Could Females Have Their Say in Choosing Their Mates?
}

\author{
Bernard Thierry ${ }^{a}$, Nancy Rebout $^{a}$, Andrea Sann $^{b}{ }^{,}$Elena Vero $^{b}$, Roberto Cozzolino $^{b}$, \\ Arianna De Marco ${ }^{b-d}$ \\ anstitut Pluridisciplinaire Hubert Curien, Université de Strasbourg, Centre National de la \\ Recherche Scientifique, Strasbourg, France; bondazione Ethoikos, Radicondoli, \\ 'Parco Faunistico di Piano dell'Abatino, Poggio San Lorenzo, and Istituto di Scienze e \\ Tecnologie della Cognizione, Consiglio Nazionale delle Ricerche, Rome, Italy \\ E-Mail: bernard.thierry@iphc.cnrs.fr
}

In mate guarding, an adult male closely follows a fertile female and mates with her over a period of several days, excluding other males from reproduction. This tactic is based on contest competition and is used by dominant males. In species characterized by a relaxed dominance style, however, it may be asked whether females have their say in the choice of sexual partners. To answer this question, we studied 16 females at the time of œstrus in 6 captive groups of Tonkean macaques (Macaca tonkeana). We found that in most instances the guarding male was neither the female's best friend nor the female's most-sought after partner at the time of ostrus, but the highest-ranking male of the group. This means that mate choice was mainly decided by the outcome of male-male competition. However, a case of the highest-ranking male being challenged by a coalition of other males has been observed, in which case the female was able to choose one of the latter individuals as her mating partner. These results show that mate choice primarily depends on the balance of power between males in Tonkean macaques. Whereas dominant males are responsible for the great majority of matings, thanks to sexual coercion, opposition from male parties to dominant males can create an opportunity for females to choose their mate. This research complied with the European Directive 2010/63/EU.

\section{Habitat Characterization of the Proboscis Monkey (Nasalis larvatus) along the Kinabatangan River, in Sabah, Borneo: A First Field Mission}

\author{
Valentine Thiry ${ }^{a, c}$, Danica Stark ${ }^{b}$, Benoit Goossens $s^{b}$, Roseline Beudels-Jamar ${ }^{c}$, \\ Régine Vercauteren Drubbel ${ }^{a}$, Martine Vercauteren ${ }^{a}$

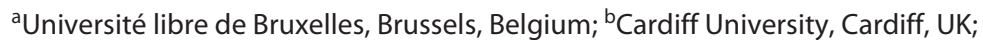 \\ 'Royal Belgian Institute of Natural Sciences, Brussels, Belgium \\ E-Mail: valentinethiry89@gmail.com
}

The proboscis monkey, Nasalis larvatus, is a large arboreal colobine primate endemic to the island of Borneo, where natural forests have been and continue to be massively logged to be converted into agricultural lands, mostly for the palm oil industry. In Sabah (north-east Borneo), clearing activities lead to heavily fragmented and degraded landscapes, nowhere so visible than along the main watercourses, such as the Kinabatangan River. Habitat loss and degradation threaten many species, among which is the proboscis monkey, now classified as 'Endangered' (IUCN, 2014). The Kinabatangan River region is one of the five major centres of continuous population of proboscis monkeys in Sabah. It is crucial that future conservation programmes endeavour to establish additional protected areas and ensure connectivity between suitable habitats. With this objective in mind, further knowledge on the proboscis monkey's eco-ethology is essential. My PhD objectives are (1) to determine the most important tree species for the proboscis monkey, by studying its diet through the analysis of faeces using the DNA metabarcoding method, (2) to understand habitat use in relation to the nutritional quality of the available foliage and the structural characteristics of the habitat and (3) to investigate the role of the proboscis monkey in seed dispersal and forest regeneration in such a fragmented ecosystem. 


\title{
Behavioural Flexibility versus Morphological Stasis? Heritability of Behavioural Traits Relative to Morphological and Functional Traits
}

\author{
Pauline Thomas, Emmanuelle Pouydebat, Fabienne Aujard \\ UMR 7179 MECADEV, Muséum National d'Histoire Naturelle, Paris, France
}

E-Mail: pthomas@mnhn.fr

The transmission of morphological, physiological and behavioural traits from one generation to another is of interest in evolutionary biology and has been extensively studied through trait heritability as well as quantitative trait loci studies. The heritability of a trait is considered to be one of the most important properties (Falconer \& Mackay, 1996) in quantitative genetics, and permits one to predict statistically the distribution of a phenotype in a population in which the phenotypes of the reproducers are known. Even though the heritability of many characters has been studied during the four last decades, few studies have tried to compare the heritability of suites of traits of different types in the same population (e.g. behaviour, physiology, morphology). My project aims to test whether a trait with a greater phenotypic variability such as behaviour is less heritable than associated morphological or functional traits in a small primate: the grey mouse lemur (Microcebus murinus). For example, the dimensions of the head and bite force are traits associated with aggressive behaviour and should evolve in the same direction, knowing that head morphology is associated with bite force performance and that aggressive behaviour is likely also related to bite force. The so called 'animal model' will be used to compare whether the transmission of different traits shows similar patterns in term of transmitted genetic and nongenetic variances. Moreover, this quantitative approach will enable us to identify genetic correlations between these traits.

\section{Mating Patterns in Relation to the Timing of Ovulation in Argentine Black Capuchins}

Barbara Tiddi $^{a, b}$, Brandon C. Wheeler ${ }^{c}$, Michael Heistermann ${ }^{d}$

${ }^{a}$ Courant Research Centre Evolution of Social Behaviour, Georg-August Universität, and

${ }^{b}$ Cognitive Ethology Laboratory, German Primate Centre, Göttingen, Germany;

'School of Anthropology \& Conservation, University of Kent, Canterbury, UK;

'Endocrinology Laboratory, German Primate Centre, Göttingen, Germany

E-Mail: batiddi@gmail.com

Tufted capuchin (Sapajus spp.) females have been shown to strongly prefer the alpha male and direct most of their solicitations to this individual. The strength of this preference, however, varies across populations of tufted capuchins and copulations with other males occur in all studied populations. Females thus mate promiscuously, and a focus on the timing of mating with alpha versus other males is crucial to understand female mating strategies better and, in turn, their potential influence on male reproductive skew. However, these questions have been largely unaddressed due to the lack of data matching the timing of ovulation with timing of copulations in wild populations. Here, we examined female mating preference with a specific focus on the distribution pattern of copulations with different males in relation to the timing of ovulation. During four mating seasons, we observed 150 copulations with 17 females belonging to three groups of black capuchins (Sapajus nigritus) in Iguazú National Park, Argentina. Although females showed high preference for the alpha male, 12 out of 17 females also mated with other group males ( $\mathrm{n}=55$ copulations). When considering the timing of ovulation as assessed by faecal progestogen measurements, copulations were highly synchronized with the female fertile phase and copulations with alpha males tended to occur significantly closer to ovulation compared to other males. In addition, Hinde index values showed that females were actively responsible for 
maintaining proximity to the target male during their proceptive periods. However, Hinde index values did not increase with the approach of ovulation, suggesting that females' contribution to proximity maintenance was not strictly linked to the timing of ovulation. Overall, females in our study groups seem to show a combination of strategies that aim at both biasing paternity to the alpha male and confusing paternity among group males. Our research complied with the European Directive 2010/63/EU.

\title{
Good Neighbourly or Teeth Grinding: Song Mediates the Interaction of Indri Groups in the Forest
}

\author{
Valeria Torti ${ }^{a}$, Marco Gamba ${ }^{a}$, Giovanna Bonadonna ${ }^{a}$, Rose Marie Randrianarison ${ }^{b}$, \\ Cristina Giacoma ${ }^{a}$ \\ aDipartimento di Scienze della Vita e Biologia dei Sistemi, Università degli Studi di Torino, \\ Torino, Italy; ${ }^{b} \mathrm{GERP}$, Groupe d'Etude et de Recherche sur les Primates de Madagascar, \\ Fort Duchesne, Ankatso, Antananarivo, Madagascar \\ E-Mail: valeria.torti@unito.it
}

Group territoriality is associated with aggressive intergroup encounters in many monogamous primates. Family groups of indris (Indri indri) move throughout small, exclusive territories, maintain the same locations over years, defend stable areas and actively advertise their presence. Males and females sing together in coordinated songs, conveying information about territorial occupancy and localization. To clarify the role of singing in regulating intergroup relationships, we investigated whether wild indris respond differently to naturally occurring and broadcast calls of neighbour and stranger groups. We also recorded whether playback signals elicited particular behavioural responses (e.g. alarm, vigilance, scent-marking, approaching/moving away from the speaker, resting, scratching) finding support for the intergroup spacing hypothesis. We conducted playback sessions $(n=127)$ on three groups inhabiting the Maromizaha rainforest, in Madagascar, between September 2011 and October 2014 In terms of the overall song duration, unit types emitted, duration or spectral parameters of the notes, we did not find any statistically significant differences among the vocal responses to natural and playback songs. However, exposure to playbacks elicited vocal ( $84.5 \%$ of cases) and behavioural/spatial responses (100\%). Orientation toward the speaker, vigilance, scent-marking, singing and group movement following playbacks were all statistically significantly $(0.001<\mathrm{p}<0.005)$. The research complied with the European Directive 2010/63/EU and the International Primatological Society (IPS) Guidelines for Use of Non-Human Primates in Research; research permits have been obtained from the MEF since 2004

\section{Cracking the Orangutan Code: An Alternative Form of Meaning Encoding}

Simon W. Townsend ${ }^{a}$, Brigitte Spielmann $^{b}$, Carel van Schaik ${ }^{b}$, Balthasar Bickel $^{c}$

anstitute of Evolutionary Biology and Environmental Studies, University of Zurich,

${ }^{\mathrm{b}}$ Anthropology Institute and Museum, University of Zurich, and ' Department of

Comparative Linguistics, University of Zurich, Zurich, Switzerland

E-Mail: simon.townsend@ieu.uzh.ch

Despite its importance, much contention still surrounds how and, critically, when the capacity to combine sounds evolved in humans. Comparative research in animals, particularly our closest living relatives, the primates, can serve as a useful tool to elucidate the evolutionary origins and early proto-forms of these abilities. Whilst an emerging body of work is beginning 
to suggest a number of monkey species are capable of combining vocalizations together into larger meaningful structures, the picture is complicated by the apparent absence of similar combinatorial abilities in the communication system of great apes. This represents a key hurdle when attempting to reconstruct the evolution of the human capacity for combinatoriality. It could be, however, that apes possess the capacity to combine sounds into larger meaningful structures but implement an alternative mechanism to achieve this. We focused on the long calls of orangutans: extended vocal constructions that are composed of acoustically distinct call units arranged in a stereotyped fashion. Previous research has demonstrated that long calls are produced in different behavioural contexts (spontaneous and elicited contexts) and receivers can differentiate between them, but the underlying cause responsible for meaning differentiation remained ambiguous. We analysed the internal structure of 70 long calls from 6 orangutans and could show that the feature potentially serving to structurally distinguish orangutan long calls produced in different contexts is the proportion of specific sound elements within the sequence. To our knowledge, no analogue for proportional encoding of meaning exists in human language. We argue that these data, therefore, represent a currently undocumented probabilistic mechanism underlying meaning generation in animals and support the suggestion that the capacity to combine sounds together was likely in place before language fully evolved.

\title{
Psychopathologies in Pet and Performing Chimpanzees: Diagnosis, Therapy, Legal Regulation and Awareness
}

\author{
Yulán Úbeda ${ }^{a, b}$, Miquel Llorente $e^{a, c}$ \\ aUnitat de Recerca i Etologia, Fundació Mona, Riudellots de la Selva, ${ }^{b}$ Facultat d'Educació \\ i Psicologia, Universitat de Girona, Girona, and 'Institut Català de Paleoecologia Humana i \\ Evolució Social (IPHES), Tarragona, Spain \\ E-Mail: yulanubeda@hotmail.com
}

We present the theoretical approach for the diagnosis of psychopathologies in chimpanzees, the results that have been achieved on the application of therapy and some future directions regarding legal regulations and awareness. So far, studies of psychopathology in non-human primates are scarce and have been focused mainly on chimpanzees used in biomedical research. Therefore, it is necessary to examine if these studies can equally be applied to pet and performing chimpanzees. But first of all, to make a correct diagnosis, it is important to create the appropriate tool. Up to now, the few studies that have been undertaken have basically used a human tool: Diagnostic and Statistical Manual of Mental Disorders. If we decide to use this tool, it is really important to adapt it for a correct assessment in these other species. In addition, although many studies have been conducted on the welfare sector, those that could be considered to be therapy are still very scarce. In this approach, it is very important to take into account the possible mental disorder of the animals in order to enhance their welfare. For that reason, it is still necessary to create psychotherapeutic protocols that demonstrate their effectiveness. We believe also, that diagnosing psychopathologies in these chimpanzees will contribute to providing ethical fundaments to raise public awareness; consequently it would promote the ending of these procedures. In addition, the diagnosis would be used to provide arguments to create a legislative regulation regarding such uses. Lastly, is important to mention that these studies can provide results from a phylogenetic and comparative perspective of human psychopathology. In conclusion, the creation of a tool for the assessment of psychopathologies in pet and performing chimpanzees is important not only for making a correct diagnosis of mental disorders, but also for the enhancement of welfare regarding the therapies as well as for the application of legal regulation and awareness. This research complied with the European Directive 2010/63/EU and the International Primatological Society (IPS) Guidelines for the Use of Nonhuman Primates in Research. 


\title{
The Role of Highly Selected Foods in the Diet of Wild White-Faced Capuchin Monkeys (Cebus capucinus)
}

\author{
Bernardo Urbani ${ }^{a}$, Paul A. Garber ${ }^{b}$ \\ ${ }^{a}$ Centro de Antropología, Instituto Venezolano de Investigaciones Científicas, \\ Caracas, Venezuela; ${ }^{b}$ Department of Anthropology, University of Illinois, \\ Urbana-Champaign, Urbana, III., USA \\ E-Mail: bermardourbani@yahoo.com
}

Patterns of foraging and diet selection are fundamental for understanding primate feeding ecology. This research explores the diet of a group of white-faced capuchin monkeys (Cebus capucinus). Data were collected in 2006 in a rainforest in northeastern Costa Rica during an 8 month period (naturalistic study) and during an additional 3 month period of an experimental field study. The monkeys visited 306 feeding/resting trees, and spent $57 \%$ of feeding/foraging time on plant parts, and $43 \%$ of feeding/foraging time on animal prey. We found that the top 6 tree species (Dipteryx panamensis, Bactris gasipaes, Ficus americana, F. insipida, Inga spectabilis, Nephelium lappaceum) comprised 53\% of the total plant feeding time. These tree species occurred at a density of $\leq 5.0$ individuals/ha. These tree species fruited during different months of the year and provided the capuchins with a stable year-round fruit crop. We refer to the feeding pattern adopted by the capuchins in terms of the exploitation of 'highly selected foods' (HSFs). HSFs are defined as food resources that represent $\geq 50 \%$ of the total yearly and monthly plant feeding time, but account for $\leq 10$ plant species in the forest. Given that HSFs were available during all months of the year and exhibited a highly scattered distribution across their home range, we examined capuchin travel patterns and cognitive skills associated with locating/revisiting these feeding sites. The role of HSFs requires further exploration among primate taxa. This study complies with the International Primatological Society (IPS) Guidelines for the Use of Nonhuman Primates in Research.

\section{The Social System of Homo erectus: Inferences Based on Patterns in Extant Primates}

C.P. van Schaik, E.P. Willems

University of Zurich, Zurich, Switzerland

E-Mail:vschaik@aim.uzh.ch

Paleoanthropologists have long asked questions about the social system of extinct hominins. Primatology's ability to answer these questions has been notoriously limited. Here, we use comparative data on extant non-human primates to reconstruct the social system of Homo erectus, based on the fact that this species lived on the open savannah, despite the presence of various large carnivores, and ate the meat of large vertebrate prey. We first show through comparative analysis that the classic inference of large group sizes was incorrect because the effect of terrestrial locomotion was confounded with that of habitat. We then show that species that live on the open savannah differ in that males confront (counter-attacking) predators instead of groups simply fleeing and mobbing. We predict that the feasibility of confrontation depends on the predatorprey size ratio, effectiveness of weaponry and number of males. Primate data support this prediction. Thus, savannah life was made possible by effective male-bonded, weapons-based defence against large carnivores. We therefore conclude that $H$. erectus lived in very large, and often cohesive, groups, presumably with endogamy of both sexes and male-female friendships. Confrontational defence easily leads to the new lifestyle of confrontational scavenging. Extant primate data can therefore contribute to reconstructing hominin lifestyles.

374

Folia Primatol 2015;86:235-386 DOI: $10.1159 / 000435825$ 6th European Federation for Primatology Meeting 


\title{
New Environment, New Possibilities, a Case of Re-Socialization and Its Effect upon Spatial Use and Social Behaviour
}

\author{
L.I. Vendrell, S. Climent, V. Benítez, M. Llorente \\ Fundació MONA, Campllong, and Universitat de Girona, Girona, Spain \\ E-Mail: lluveci@gmail.com
}

One of the main challenges primate rescue centres face is the continuous ingress of new apes. Primate abuse and illegal trade of animals is a reality in Europe and the result is that primates often end up in primate rescue centres and sanctuaries. They exhibit behavioural problems and lack important social skills for living in a group environment. Socialisation, re-socialisation and their introduction to previously established groups are some of the management techniques used to promote the development of species-typical behaviours. One of the most relevant factors to enhance welfare is the outdoor facility where these apes will interact. Our objective was to identify the zones where they spent most of their time and see if these zones changed during the re-socialization process. In addition, we observed the behavioural changes that occurred during the first three months after their complete integration into a group. To provide objective measures of animal welfare, we used a multidisciplinary approach. The spatial data were recorded using the scan sampling technique and the behavioural data were recorded using the focal sampling technique. The study was carried out at Fundació Mona with a sample of 8 chimpanzees (5 from an old group and 3 new members). The total duration of the study was 99 days (February 2014 to June 2014) and the data were divided into three phases of the same number of days. The results suggest that: (1) after a period of adaptation during phase 1, the most significant effect was the preference for core activity areas that elicited social activities in the whole group. (2) Social positive behaviours as well as the re-socialization index increased over time. We conclude that spatial data are relevant for understanding how the primates responded to their new environment, but we would have to undertake a long-term study to find out if the apes were completely integrated into the group.

\section{Processing Caustic Cashew Nuts: Factors Affecting Success in Wild Bearded Capuchin Monkeys (Sapajus libidinosus)}

\author{
Marialba Ventricelli ${ }^{a}$, Elisabetta Visalberghi ${ }^{a}$, Alessandro Albani ${ }^{a, b}$, Gabriele Schino $^{a}$
}

astituto di Scienze e Tecnologie della Cognizione, Consiglio Nazionale delle Ricerche, and

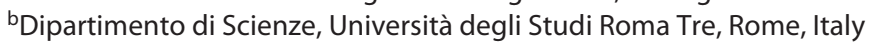

E-Mail: marialbaventricelli@gmail.com

Only a few primate species process food before ingestion to avoid caustic protective substances. We investigated how wild bearded capuchin monkeys (Sapajus libidinosus) living at Fazenda Boa Vista (Piauí, Brazil) process cashew nuts (Anacardium spp.) in order to eat the kernel while avoiding the caustic chemicals contained in the mesocarp. To assess the role of experience, sex and body mass on success and processing behaviour, we recorded the behaviour towards fresh $(\mathrm{n}=1,282)$ and dry cashew nuts $(\mathrm{n}=532)$ of 23 individuals of different age-sex classes. Capuchins were significantly more successful in opening fresh nuts than dry nuts ( 87.4 and $72.6 \%$ of the nuts, respectively), whereas total processing time did not differ. In both cases, experience and body mass affected success whereas sex did not. Infants never succeeded. Capuchins, especially adults, use different sets of behaviours to process fresh and dry nuts. Success was more likely if the monkey rubbed the fresh nut, or if it used a percussive tool on the dry nut. Overall, signs of discomfort (e.g. salivation or scratching directed to the face or mouth) were rare; whereas they are common in other populations that process the nuts differently. Young monkeys did not look closely at the processing done by adults, nor did adults intervene in any way in what youngsters did. Therefore, whereas individual experience and body mass play important roles in tuning the skills to access

6th European Federation for Primatology Meeting
Folia Primatol 2015;86:235-386 DOI: $10.1159 / 000435825$ 
a nut, observing expert group members seems insignificant. The above processing techniques have been observed only in our site and capuchins elsewhere present aversive responses to cashews rather than ingestion. Therefore, future studies should investigate other capuchin populations living in areas where cashews are native and abundant to verify whether cashew processing can be considered a cultural phenomenon. We adhered to the International Primatological Society (IPS) Guidelines for the Use of Nonhuman Primates in Research. Permission to work in Brazil was granted by IBAMA and CNPq.

\title{
Monkeys for the Pope: Presence, Importance and Importation of Non-Human Primates in Renaissance Italy (15th and 16th Centuries)
}

\author{
Veracini Cecilia, Catarina Casanova \\ CAPP - ISCPS, University of Lisbon, Lisbon, Portugal \\ E-Mail: cveracini2011@gmail.com
}

In the Age of Discovery, Europeans came into direct contact with sub-Saharan African and New World primates for the first time. Recent evidence suggests they were introduced to Europe in greater numbers than originally assumed, reaching most of the European countries at that time. Italian rulers had a strong tradition of keeping exotic animals for their image and primates represented expensive pets for nobles and clerics who used them to underscore their influence, prestige and social position. Many of them were portrayed and their artistic representation provides us with information on their occurrence in Italy that would otherwise not have been documented. The current work presents the results of a review of literary and iconographic sources of this period, which contain data on African and New World primates in Renaissance Italy. The embassy sent to Pope Leo X by the Portuguese king Manuel I in 1514 enriched the Pope's menagerie with many exotic animals. Among them there were the golden capuchin monkey (Sapajus flavius), the Sabaeus monkey (Chlorocebus sabaeus) and a drill (Mandrillus leucophaeus) portrayed by Raffaello Sanzio in the Logge Vaticane. Moreover this species was depicted later in the Sant'Angelo Castle (1520-1540) together with a grivet monkey (Chlorocebus aethiops). Other species, such as Callithrix jacchus, Callithrix geoffroyi, Sapajus xanthosternos, Sapajus cfr. libidinosus, Cebus capucinus, Macaca sylvanus, Papio anubis, Papio hamadryads and probably specimens of the genus Ateles, were portrayed in other noble residences or in other contexts in many Renaissance Italian states. These representations are often the first scientific description of a particular species we have. Integrating historical and iconographic data allows us to understand the original distribution of many species, make inferences about the consequence of this trade on primate populations and produce useful information for their conservation management.

\section{Perceptions of Primates in Mexico: How Do People Use Monkeys?}

Francisca Vidal-García, Juan Carlos Serio-Silva

Red Biología y Conervación de Vertebrados, Instituto de Ecología A.C., Xalapa, Veracruz, México

E-Mail: frany01@gmail.com

Human perception of monkeys is the consequence of the kind of contact that people have with them in the areas that these animals inhabit. We have been working to understand the opinions of local people about the three species of Mexican monkeys (Alouatta pigra, A. palliata and Ateles geoffroyi). We interviewed 1,350 people in 375 localities in Mexico. The interviews were based on 15 questions about personal knowledge of the primates concerning their biology and behaviour, local uses, the benefits obtained from them and the interest in primate conservation. 
At present, we have identified tendencies using descriptive statistics. We found 219 places with current presence, 94 places with past presence and 62 places in which the species have never been present. Attitudes and uses depended on the places in which people were living. Negative opinions were more frequent in places in which people do not have contact with monkeys. However, knowledge and positive perceptions were more frequent in places in which people have, or had, daily contact with them. We have collected information on 19 traditional uses for monkeys; these include nine recipes and three medicinal uses. People have identified 67 different plants which monkeys use in their diet. These results represent a first approximation for identifying perceptions about monkeys and, therefore, places in which conservation efforts could be effective.

\section{The Current Distribution of Primates in Mexico: Models as Tools of Conservation}

Francisca Vidal-García, Juan Carlos Serio-Silva

Red Biología y Conervación de Vertebrados, Instituto de Ecología A.C., Xalapa, Veracruz, México

E-Mail: frany01@gmail.com

We developed potential distribution models for all three species of primates which are distributed around southeastern Mexico (Alouatta pigra, A. palliata and Ateles geoffroyi) and which are classified as endangered species. The models of potential distribution and a map of the vegetation in which monkeys have been recorded were combined to obtain the models of expected presence-absence. We considered the following two conditions for the presence-absence models: (1) if both characteristics were present, then the species would be present; and (2) if one of these characteristics was absent, then the species would be absent. Then 375 localities around southeastern Mexico were chosen (32 in Quintana Roo, 76 in Campeche, 10 in Yucatan, 81 in Tabasco, 68 in Chiapas, 50 in Veracruz and 58 in Oaxaca) and visited for verifying presence-absence of species. These localities had not been visited before by other researchers. We found 219 localities with current presence of some species of primates (41 with A. palliata; 65 with A. pigra, 34 with A. geoffroyi, 64 with A. pigra and A. geoffroyi, 13 with A. palliata and A. geoffroyi and 2 with A. pigra and $A$. palliata sharing the geographic space). We found new evidence about the northern limits of distribution of primates in Mexico and in America. Our main contribution is new knowledge and information about the distributional limits of primates in Mexico, local threats and a method to verify models of potential distribution by field visits.

\section{The Bearded Capuchin Monkeys of Fazenda Boa Vista}

\section{Elisabetta Visalberghia, Alessandro Albanib}

a Istituto di Scienze e Tecnologie della Cognizione, Consiglio Nazionale delle Ricerche, Rome, Italy; ${ }^{b}$ Dipartimento di Scienze, Università degli Studi Roma Tre, Rome, Italy Email: elisabetta.visalberghi@istc.cnr.it

This documentary illustrates ten years of research on the ecology, social behavior and the tool use skills of two groups of wild bearded capuchin monkeys living in the dry forest habitat of Fazenda Boa Vista (Piauí State, Brazil). The documentary aims (i) to present to a large audience the discoveries made by the researchers of the EthoCebus team (www.ethocebus.net) of capuchins, and (ii) to provide a rigorous/scientific interpretation of capuchins behavior. Its main part (lasting about $40 \mathrm{~min}$ ) provides a general overview of the ecology and behavior of this population; it also pays special attention to the use of stone tools to crack open encased food, since this makes the Fazenda Boa Vista population particularly worth studying. Another part of the documentary (also lasting about $40 \mathrm{~min}$ ) illustrates (i) the beginning of the research, (ii) the innovative research 
and field experiments carried out to investigate stone tool use and (iii) how humans co-existed with wild animals as well as the recent threat posed by deforestation and intensive agriculture that are approaching the homerange of our population. We see our attempt to shoot capuchins in the wild with a low-budget as an example of how researchers should contribute to the quality of a documentary and its ethics by being actively involved from the start to the very end of it.

\title{
Characterizing Vocal Repertoires - Shortcomings of Current Methods and Future Perspectives
}

Philip Wadewitz ${ }^{a-c}$, Kurt Hammerschmidt ${ }^{a}$, Julia Fischer ${ }^{a}$

${ }^{a}$ Cognitive Ethology Laboratory, German Primate Center, ${ }^{\mathrm{b}}$ Max Planck Institute for Dynamics and Self-Organization, and 'Bernstein Center for Computational Neuroscience, Göttingen, Germany

E-Mail: pwadewitz@dpz.eu

Vocal repertoires serve as a critical foundation to investigate how acoustic communication systems evolve and they provide the basis for comparative analyses among individuals, populations and taxa. However, since there is currently no standard in the methodology used to characterize repertoires, comparisons within and between species remain difficult. Two of the major obstacles are (a) the measurement of different acoustic features in different studies and (b) the insufficiency of current methods to quantify the graded nature of vocal repertoires. In this project, we address these challenges, comparing two datasets of previous recordings made from wild chacma baboons (Papio ursinus) in the Okavango Delta, Botswana and from semi-wild Barbary macaques (Macaca sylvanus) in an outdoor enclosure in Rocamadour, France. To assess the influence of feature selection on the classification results, we first constructed different sets of acoustic features from the chacma baboon dataset and validated the resulting classifications. Our findings show that datasets with a higher number of acoustic features lead to better clustering results than datasets with only a few features. The use of extracted factors in the cluster analysis resulted in an extremely poor resolution of emerging call types. To quantify the graded structure of the two datasets, we used fuzzy clustering. This approach reveals typical and atypical calls by assigning membership values for each call type to every call. Whereas typical calls share most properties with a single call type, atypical calls represent intermediate forms and share properties between multiple call types. The results reveal a significantly higher level of gradation in the Barbary macaque repertoire compared to the chacma baboon repertoire. These details about the structure of vocal repertories cannot be captured with classical clustering approaches. Research complied with the IPS Guidelines for the Use of Nonhuman Primates in Research.

\section{Two Cases of Dead-Infant Carrying Followed by Mother-Infant Cannibalism in Captive Socially Housed Japanese Macaques}

\author{
Claire F.I. Watson*, Naoko Hashimoto*, Natsume Takayoshi, Munehiro Okamoto, \\ Tetsuro Matsuzawa \\ Primate Research Institute, Kyoto University, Kyoto, Japan \\ *The two first authors contributed equally to the research. \\ E-Mail: cfi.watson@gmail.com
}

Interest is growing in attempts to understand animal behaviour towards dead conspecifics, especially among non-human primates. Although rare, infant-corpse-carrying is evident in many non-human primate species. However, in Japanese macaques (Macaca fuscata) it is relatively 
common, on average lasting several days. The combination of dead-infant-carrying for at least a day followed by filial cannibalism has been reported in one wild bonobo and two rehabilitant orangutan mothers. We report the first instances of dead-infant carrying followed by filial cannibalism in a monkey, housed socially. We present two cases of infant-corpse-carrying for 4 weeks, then filial cannibalism in a female Japanese macaque in two consecutive births. The individual (wild-born) was one of 25 individuals housed in a large outdoor enclosure $\left(3,800 \mathrm{~m}^{2}\right)$ with natural vegetation. We discuss the implications of our findings. Our observations are also unusual for the exceptionally long period of infant-corpse-carrying. On both occasions, the mother carried the corpse until mummified: in 2011 for 29 days and in 2013 for 28 days. In 2011 she consumed all the dried flesh on day 28 , yet continued carrying the skeletal remains intermittently for another day. In 2013 she chewed the corpse on day 23. This research complied with the Kyoto University Primate Research Institute Guidelines for Care and Use of Nonhuman Primates and with the International Primatological Society (IPS) Guidelines for the Use of Nonhuman Primates in Research.

\title{
Public Perceptions of Threatened Slow Lorises (Nycticebus spp.) on Web 2.0 Sites and Implications for Social Media Reporting Policies
}

\author{
Siobhan Webster ${ }^{a}$, Louisa Musing ${ }^{b}$, Asier Gil Vazquez ${ }^{b}$, K.A.I. Nekaris ${ }^{b}$
}

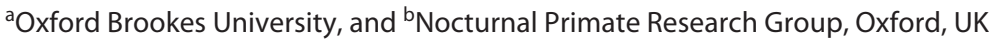

E-Mail: siobhan.webster-2014@brookes.ac.uk

Slow loris (Nycticebus spp.) populations are heavily threatened by illegal acquisition for the pet trade. Videos of pet lorises repeatedly go viral on social media sites where there is a lack of reporting mechanisms for animal cruelty or illegal animal activity. The videos show lorises kept in unsuitable conditions and have perpetuated misconceptions of their threatened status and suitability as pets. We analysed 93 videos on social media sites of pet lorises for the presence of conditions that violated animal welfare according to the five animal freedoms. Conditions included ill health, daylight and isolation; $100 \%$ contained at least two conditions whilst at least four conditions were present in $92.5 \%$ of videos. We also launched a petition 'Add a button to report Animal Abuse and Illegal Animal Activity to Facebook posts', which at the time of writing had 2,568 signatures. We analysed 337 comments on the petition, determining common themes: $25.8 \%$ mentioned animal abuse, $10.6 \%$ referred to the direct need for a reporting button and $7.2 \%$ suggested Facebook enables animal abuse to be shared. Following these results, an online survey will be distributed to (a) assess whether statements related to slow lorises would (i) dissuade participants from viewing a video, and (ii) encourage participants to report a video; and (b) investigate participants' experience with reporting inappropriate content to social media sites. Full results from this survey will be presented, with preliminary results showing that 83 and $89 \%$ of participants who have previously watched a video of a pet slow loris would have been discouraged from watching, and encouraged to report the video, respectively, if it was accompanied by any one of the five statements offered. These results indicate the need for social media sites to improve reporting policies for animal cruelty and illegal animal activity, and to demonstrate to site users that appropriate action will be taken. 


\section{Reinforcing the Java Silvery Gibbon Population in the Mount Tilu Nature Reserve, West Java, Indonesia}

\section{Made Wedana}

The Aspinall Foundation Indonesia Programme, Desa Alam Endah, Kec. Rancabali Bandung, West Java, Republic of Indonesia

E-Mail: madewedana@ hotmail.com

The Java silvery gibbon (Hylobates moloch) is listed by the IUCN as Endangered, with the reasons for population decline identified as the illegal pet trade, hunting and loss and fragmentation of habitat. Populations of the species occurring in West Java and Central Java live in isolated forest fragments and, in many of these, appear to be at low densities or, in some cases, extinct. Here we present the preliminary results of the Mount Tilu Java silvery gibbon reinforcement project. The aim of this project is to re-establish a viable, self-sustaining population of the Java silvery gibbon in this forest reserve. This is being done through the release of rehabilitated confiscated and donated gibbons held at the Java Primate Rehabilitation Centre (JPRC) in West Java. Two adult rehabilitated pairs were reintroduced in 2014 and followed by means of direct observation. In the first year of the monitoring period, the adult male from the first pair was attracted by a wild female and remains paired with this wild female. The adult female from the second pair went missing two months after the reintroduction. Based on those two cases, radio tracking is now being used to improve monitoring of the reintroduced gibbons.

\section{Constraints Associated with Living in a Savannah-Woodland Environment: Seasonal Stress Patterns in Chimpanzees (Pan troglodytes verus) at Fongoli, Senegal}

Erin G. Wessling ${ }^{a}$, Hjalmar Kuehl' ${ }^{a}$, Tobias Deschner ${ }^{a}$, Jill D. Pruetz ${ }^{b}$

aDepartment of Primatology, Max Planck Institute for Evolutionary Anthropology,

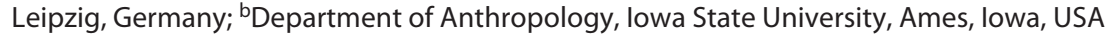

E-Mail: erin_wessling@eva.mpg.de

The Fongoli chimpanzee (Pan troglodytes verus) research community is expected to endure the hottest and driest conditions within the biogeographical range of the species. Rainfall at Fongoli averages $900 \mathrm{~mm}$ annually which is confined to only four wet season months, while temperatures frequently exceed $40^{\circ} \mathrm{C}$. Understanding how this environment affects our closest living relative is crucial to our understanding of the evolutionary pressures associated with living in such an environment. Here, we test the hypothesis that Fongoli chimpanzees are heat, water and nutritionally stressed on a seasonal basis. This is the first study to directly link seasonal variation in temperature, water and food availability to physiological biomarkers of stress in chimpanzees from a savannah-woodland habitat. We analysed over 300 urine samples, collected non-invasively from adult individuals in the Fongoli community, for cortisol, creatinine and c-peptide as measures of heat, water and nutritional stress, respectively. We have explored seasonal patterns in the aforementioned hormones to examine if Fongoli chimpanzees experience periods of seasonal stresses associated with climatic and phenological variation, and if these stress patterns are more pronounced than patterns of stress markers from chimpanzees living in more forested habitats. This research provides new insights into constraints associated with living in a savannah environment, and the physiological and biogeographical consequences that these constraints have for chimpanzee ecology, as well as hominin evolution. This research conforms to the ethical research standards set by the Department of Primatology at MPI-EVA and the International Primatological Society Guidelines for Use of Nonhuman Primates in Research. 


\title{
Ambush Predators May Select for Small Group Size in Rainforest Primate Prey
}

\author{
Brandon C. Wheeler ${ }^{a}$, Charles H. Janson ${ }^{b}$
}

aSchool of Anthropology and Conservation, University of Kent, Canterbury, UK;

${ }^{b}$ Division of Life Sciences, The University of Montana, Missoula, Mont., USA

E-Mail: bcwheeler43@gmail.com

Classic ecological models of social groups suggest that increasing group size typically leads to a decrease in both individual predation risk and net food intake, with optimal group size being a compromise between these benefits and costs. Among rainforest primates, the main antipredator benefits of sociality are thought to result from the dilution effect and collective detection of predators. However, recent research suggests that vegetation density in rainforest habitats limits the benefits of collective detection against ambush predators such as raptors, felids and snakes. Further, while larger groups are acknowledged to be more conspicuous to predators, it is widely assumed that this cost is unlikely to outweigh dilution benefits. Here we show in a simple model that per-individual rates of both encounters with predators and successful ambush attacks per encounter can increase with group size, under conditions likely to hold for many primate groups (when increases in group size lead to increases in group spread, conspicuousness and daily travel distance). Consequently, individual risk against ambush predators that employ a sit-and-wait strategy to search for prey, such as many snakes and some raptors, is lowest in small to mediumsized groups. In contrast, individuals in relatively large groups are favoured against ambush predators like felids that employ a cruising strategy to search for prey, although even in this case increasing group size above some threshold increases individual risk. These results suggest that maximum group size among primates can be limited by increasing predation risk. Research focused on primate predators is needed to determine the extent to which the model accurately reflects their behaviour.

\section{Factors Influencing the Body Weights of Captive Lemur catta in UK Institutions}

Clare White, Giuseppe Donati

Oxford Brookes University, Oxford, UK

E-Mail: clarecjw16@hotmail.com

Captive Lemur catta are susceptible to obesity due to their diet and their reduced activity compared to those in the wild. Obesity causes health issues including reproductive problems, cardiovascular disease and diabetes - it is therefore vital to know influencing factors and preventative measures. Captive individuals are classified as overweight or obese compared to wild weight averages. Food intake and activity pattern observations were conducted to determine differences in amounts consumed, items consumed and time active between individuals. A questionnaire was distributed to UK institutions, exploring factors potentially influencing body weight, such as age, gender, enclosure type, group size, night access and browse presence, and to determine captive weight ranges. No relationship was found between body weight and total grams consumed, and no individual consumed near the average provisioned. There was a strong tendency towards a positive trend between body weight and metabolisable energy. All individuals spent more time inactive than active, with little variation between them. No correlation was found between body weight and age, group size, dominance, or presence of browse. Captive weight ranges were higher than wild ranges, standard enclosures had significantly heavier occupants, and individuals with full night access were significantly heavier than those with weather dependent access. Males were significantly heavier than females, which is unexpected given fe- 
male dominance. The average weight for captive Lemur catta was 2,603 g compared to 2,219 $\mathrm{g}$ for wild individuals, which provides a more realistic target weight and reclassifies the number of individuals regarded as overweight or obese, with long-term husbandry implications. Metabolisable energy intake was the most influential factor on body weight, but no one aspect explained the variation between individuals. Additional factors must play a role. This study emphasises the importance of conducting food intake and activity pattern observations before making any weight-induced husbandry changes. This research project complied with the European Directive 2010/63/EU and the International Primatological Society (IPS) Guidelines for the Use of Nonhuman Primates in Research.

\title{
Spatial Consequences of Between-Group Competition in Vervet Monkeys (Chlorocebus aethiops pygerythrus)
}

\author{
Erik P. Willems, T. Jean M. Arseneau
}

Anthropological Institute and Museum, University of Zurich, Zurich, Switzerland

E-Mail: e.willems@aim.uzh.ch

Competition is a fundamental component of evolution by natural selection and a pervasive feature at all levels of biological organisation. Although its causes and consequences are generally well understood at the level of the individual gene or organism, few studies have attempted to quantify the importance of competition at higher levels of biological organisation. In primatology, it is widely assumed that individual fitness is more strongly affected by within-group than between-group competition, and thus that the latter is of only secondary importance in shaping the social system of a species. Very little empirical work, however, has been able to directly quantify the potential ramifications of between-group competition on individual fitness, whereas much more is known about the importance of competitive interactions among group members. Here we present an analysis of both the short- and long-term consequences of aggressive between-group encounters on local space use intensity and the simultaneous movement trajectories of 5 neighbouring groups of vervet monkey in their natural environment in South Africa. We present findings based on approximately 50,000 GPS-relocations, collected at 30 minute intervals throughout the diurnal activity period of animals over a 12 months observation period. As such, this study represents one of the first efforts to quantify the potential fitness benefits in terms of decreased energy expenditure and priority of access to contested resources that individuals stand to gain from living in competitively more successful groups. The use and deployment of wildlife telemetry observed all relevant local, national and EU legislations, and adhered to the $3 \mathrm{R}$ principle.

\section{Habitat Use of the Endangered L. mittermeieri - Northwest Madagascar}

Leslie Wilmet $^{a-c}$, Cédric Vermeulen ${ }^{a}$, Roseline C. Beudels-Jamar ${ }^{b}$, Christoph Schwitzer $^{c}$

aUniversity of Liege - Gembloux Agro Bio Tech, Forest Management Resources axis,

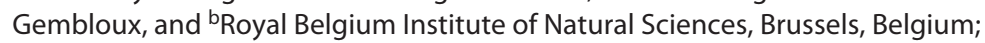
'Bristol Zoological Society, c/o Bristol Zoo Gardens, Bristol, UK E-Mail: Iwilmet@doct.ulg.ac.be

The genus Lepilemur is exemplary of the endemic mammalian fauna of the island of Madagascar. Sportive lemurs have small distribution ranges and fairly small total populations and are particularly negatively affected by deforestation and habitat fragmentation. Our research focus on a poorly-known taxon, Lepilemur mittermeieri, whose distribution is limited to the Ampasin- 
dava Peninsula in northwest Madagascar. It was described in 2006 on the basis of genetic material only. Since then, no further research has been conducted and general information on this endangered species is urgently required. We will present our global project as well as the results of a second field mission conducted from March to June 2015 in order to specifically (1) investigate habitat use of 8 radio-collared Lepilemur mittermeieri by analysis of their home range, feeding ecology and sleeping site characteristics and (2) to complete forest characterization of the home range of each radio-collared animal. This study is conducted on the Ampasindava Peninsula, a priority area for Malagasy conservation.

\title{
The Dental Morphology of Çorakyerler Hominoids (Late Miocene, Turkey)
}

\author{
Alper Yener Yavuz ${ }^{a}$, Ayla Sevim Erol ${ }^{b}$, Erhan Tarhan ${ }^{b}$ \\ ${ }^{a}$ Department of Anthropology, Faculty of Arts and Science, Mehmet Akif Ersoy University, \\ Burdur, and 'bepartment of Anthropology, Faculty of Languages, History and Geography, \\ Ankara University, Ankara, Turkey \\ E-Mail: alpyenyav@gmail.com
}

The Çorakyerler vertebrate fossil locality dating to the late Miocene is located in the Central Anatolia Region of Turkey. The fossil locality is along a road in the Yapraklı district of Çankırı province at an altitude of about 745 metres. Excavations have been held systematically in this region since 2001. The Çorakyerler vertebrate fossil locality, according to faunal and magnetostratigrafic evaluations, is dated to between MN11-12 zones (about 8-7 million years ago). The Çankırı-Çorum Basin, on which the fossil locality is located, emerged with the low tidal zone of the Tetis Sea. Within the Corakyerler fauna, the Hominoidea findings date to 7-8 million years ago. These are the youngest Hominoidea fossils yet found in Anatolia and are represented by at least four different individuals. Due to their similarities to Ouranopithecus macedoniencis, these fossils are classified as Ouranopithecus turkae. In addition to their resemblance to Ouranopithecus macedoniensis, from Macedonia and Greece, the fossils from Çorakyerler have similarities to other early Pliocene Hominoidea Australopithecus anamensis and Ardipithecus ramidus. In the excavations held since 2001, teeth and maxilla pieces have been found which belong to four different individuals. There are two mandibula pieces, one from an adult female and another from an adolescent. There is a maxilla with part of the face belonging to a young adult individual. An analysis of these maxilla and tooth morphologies promises to provide important information about the genus and species. Further, the morphology, tubercular alignment and incisive structure of the canine teeth will provide information on diet and sexual dimorphism in this hominoid.

\section{Assessing the Relative Abundance of the Mozambique Bushbaby (Galagoides granti) and the Thick-Tailed Bushbaby (Otolemur crassicaudatus) in Tshanini Community Reserve, South Africa}

Ayabulela Yokwana, Fabien Génin, Judith Masters

African Primate Initiative for Ecology and Speciation (APIES), Department of Zoology and Entomology, University of Fort Hare, Alice, South Africa

E-Mail: a.yokwana@gmail.com

Why are some animals rarer than others? The Mozambique bushbaby Galagoides granti was only recently found within the borders of South Africa in the sand forest of northern KwaZuluNatal. Moreover, the species has a relatively small geographic distribution: does this make it a 
sand forest specialist? The rarity of Galagoides granti may be explained by the ecological dominance of the more common, widespread and larger thick-tailed greater bushbaby (Otolemur crassicaudatus). We assessed the habitats and local abundances of the two species by conducting nocturnal surveys along a $9.36 \mathrm{~km}$ transect in Tshanini Community Reserve, over a total period of $40 \mathrm{~h}$. Animals used different preferred habitats: Galagoides granti was the most abundant in sand forest and dense woodland habitat while Otolemur crassicaudatus preferred relatively open woodland-savannah. However, we observed overlaps in habitat, associated with relatively frequent meeting of the two species, along forest edges and in woodland habitat. These meetings did not lead to any interaction between the two species, indicating very little competition. Furthermore, species shared some resources (gum and possibly sleeping sites), suggesting that specific adaptation rather than ecological partitioning explains their habitat preferences and geographic distributions. We conclude that Galagoides granti is rare because its favourite habitat is rare in South Africa.

\section{Pedal Grasping Modes of the Northern Smooth-Tailed Treeshrew Dendrogale murina: Insights into the Evolution of Primate Pedal Grasping}

Dionisios Youlatos ${ }^{a}$, Aleksandra Panyutina $^{b}$

${ }^{a}$ Aristotle University of Thessaloniki, School of Biology, Department of Zoology,

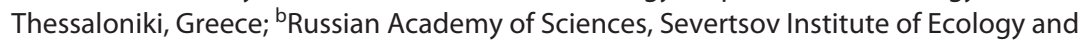
Evolution, Moscow, Russian Federation

E-Mail: dyoul@bio.auth.gr

A key primate feature is powerful pedal grasping with a divergent opposable hallux that facilitates fine branch use for foraging on angiosperm products and/or invertebrates. The evolution of pedal grasping in archontan mammals is of great importance as it bears on the adaptive significance of specialized grasping and associated behaviours. Extant non-primate arboreal mammals, with their pedal grasping diversity, constitute good models for testing putative evolutionary stages. Treeshrews, especially basal arboreal tupaiids, are very suitable for testing pedal grasping modes and associated substrate correlates. In this context, we filmed and analysed foot position, posture and grasping in three wild-caught treeshrews, Dendrogale murina, from Vietnam. Our observations showed that convergent and claw grasping were the more common pedal grasping modes in treeshrews. Hallucal grasp was used less and was mainly associated with small and vertical substrates. Convergent grasp was frequently used on medium-sized and horizontal substrates whereas claws were used on large vertical substrates. In addition, the foot was frequently inverted and mainly placed in a semiplantigrade position. Inversion and semiplantigrady dominated on small, medium-sized and horizontal substrates but decreased on larger substrates with increased inclinations. The results demonstrate that basal treeshrews do not frequently use hallucal grasping, but invert their foot and place it in a semiplantigrade position. Even though treeshrews have not yet evolved powerful opposable pedal grasping, incipient hallucal grasping was used on small and inclined substrates, underscoring the significance of a grasping hallux for fine branch use. Powerful hallucal grasping did not evolve early in archontan evolution but was pivotal for the evolution and diversification of the groups that subsequently led to primates. This research complied with Vietnamese laws and the IPS Guidelines for the Use of Nonhuman Primates in Research. 


\title{
The Power behind the Throne: Female Influence on Male Dominance Hierarchies in Vervet Monkeys
}

\author{
Christopher Young ${ }^{a}$, Richard Mcfarland ${ }^{b}$, Louise Barrett ${ }^{a, c}$, S. Peter Henzi ${ }^{a, c}$ \\ ${ }^{a}$ Applied Behavioural Ecology and Ecosystems Research Unit, University of South Africa, \\ Florida, Gauteng, South Africa; ${ }^{b}$ Department of Anthropology, University of \\ Wisconsin-Madison, Madison, Wis., USA; 'Department of Psychology, University of \\ Lethbridge, Lethbridge, Alta., Canada \\ E-Mail: Christopher.young@uleth.ca
}

Across mammalian taxa, larger, more powerful males can generally exert social dominance over females. However, in a handful of social species, in which sexual dimorphism is not extreme, females are able to win dyadic encounters against males and may even out-rank some. Where this is so, we expect females to influence the dynamics and outcomes of agonism among male competitors. We investigate this possibility in three groups of wild vervet monkeys (Chlorocebus pygerythrus) in South Africa. We firstly confirmed that some females are dominant over some males by examining male-female dyadic contests and constructing a combined dominance hierarchy. We show that females were indeed dominant over many males. We then examined the influence of the frequency of aggression from females towards males and male-female social relationships (based on frequency of affiliative behaviours) on a male's rank trajectory in the group (136 male rank trajectories from six different periods). We used Elo ratings to determine rank trajectories, with the change in Elo ratings across time serving to indicate shifts in potential male power. While the rate of female aggression against males did not influence male rank trajectories, we found that males that associated more closely with females were significantly more likely to have a positive rank trajectory than those who did not, whilst controlling for male rank. These results indicate that females can play a large role in influencing male dominance structure within vervet monkey groups and that male social investment can pay dividends.

\section{Yawning in the Forest: A Comparative Study on Wild Propithecus verreauxi and Lemur catta}

Alessandra Zannella ${ }^{a, b}$, Ivan Norscia $^{b}$, Roscoe Stanyon $^{a}$, Elisabetta Palagi $^{b, c}$

${ }^{a}$ Anthropology Laboratories, Department of Biology, University of Florence, Florence, ${ }^{b}$ Natural History Museum, University of Pisa, Calci, and ' Unit of Cognitive Primatology and

Primate Centre, Institute of Cognitive Sciences and Technologies CNR, Rome, Italy

E-Mail: Alessandra.Zannella@ hotmail.com

Yawning is an involuntary behaviour common to all vertebrates. Although easily recognizable, its adaptive significance is not yet fully understood. In the past, several physiological hypotheses have been tested to explain yawning including oxygenation, circulation and thermoregulation. More recently, researchers have begun to focus on the behavioural meaning of yawning, especially in primates. This is the first study to test hypotheses on yawning in natural populations of primates. We selected two sympatric species, Lemur catta and Propithecus verreauxi, of the Ankoba forest $\left(24.99^{\circ} \mathrm{S}, 46.29^{\circ} \mathrm{E}\right.$, Berenty Reserve) in southern Madagascar that differ in ecological and behavioural characteristics. Their low sexual dimorphism and high sexual competition may have reduced the differences between sexes in both species. As predicted from the Dimorphism Hypothesis, males and females yawned with similar frequencies. As in other primate species, yawning was linked to the sleep-wake cycle and punctuated the transitions from one behavioural pattern to another (State Changing Hypothesis supported). Finally, we tested the Anxiety Hypothesis, which predicts that yawning should increase after stressful, anxiogenic events. We found a significant increase in yawning after both predatory attacks and aggression. Clearly, primate yawning is associated with behavioural state changes within different contexts (e.g. threat,

6th European Federation for Primatology Meeting
Folia Primatol 2015;86:235-386 DOI: $10.1159 / 000435825$ 
anxious or rest). Yet it is not known if yawning is a single, unitary behaviour that varies only with respect to the context in which it is performed. We cannot exclude that yawning in lemurs can be elicited by certain type of stressors; further data in clearly defined contexts will be necessary to demonstrate this hypothesis.

\title{
The Voice of Emotion in Humans and Non-Human Mammals: Insights from a Comparative Approach
}

\author{
Elke Zimmermann ${ }^{a}$, Anna S. Hasting ${ }^{b}$, Sonja A. Kotz ${ }^{b}$, Marina Scheumann $^{a}$ \\ anstitute of Zoology, University of Veterinary Medicine, Hannover, and ${ }^{b}$ Max Planck \\ Institute for Human Cognition and Brain Sciences, Leipzig, Germany \\ E-Mail: elke.zimmermann@tiho-hannover.de
}

Across human cultures, emotions are suggested to be universally expressed and recognized in facial expressions, gestures and voice and represent a major prerequisite for decision-making in complex societies. Shared acoustic cues in non-verbal expressions, speech and music connote for the quality and intensity of an emotion and suggest a prehuman origin of affective prosody in human acoustic communication. Within this talk, we will explore the 'prehuman origin' hypothesis using a comparative approach on acoustic communication of mammals and investigate how humans recognize animals' affective states using rating by a 5-point version of the Self-Assessment Manikin (SAM). The findings indicate that affective prosody in human acoustic communication has deep-reaching phylogenetic roots. Explicit voice-induced cross-taxa recognition of emotions in human adults is, however, shaped more by experience-dependent cognitive mechanisms rather than by induced affective states or cross-taxa universal acoustic coding and processing mechanisms.

\section{Evidence for Vocal Dialects and Vocal Learning in Common Marmosets?}

Yvonne Zürcher, Judith Burkart

Anthropological Institute and Museum, University of Zurich, Zurich, Switzerland

E-Mail:yvonne.zuercher@uzh.ch

Unlike humans, most primates show only marginal or no evidence for vocal production learning, even though they use vocalizations for communication. This is even more surprising considering the vocal learning skills in humans. Members of the Callitrichidae family might be an exception amongst non-human primates in relation to vocalizations. Many species in this family communicate with a broad variety of calls, and some evidence suggests vocal production learning. The goal of this study was to assess whether common marmosets (Callithrix jacchus) have different vocal dialects that result from social learning. We therefore recorded vocalizations of three different populations of common marmosets in Zurich, Rome and Madrid, and analysed and compared the call structures. Four animals of the colony in Madrid were then transferred to Zürich and paired up with a local animal. Five additional new pairs composed of Zurich animals only were used as a control. The change of vocalizations in all animals was tracked by regular vocal recordings of each animal before and during the transfer and pair formation. During the pair formation process, we also collected behavioural data in order to quantify the influence of relationship quality on vocal assimilation. Changes in the call structure of the imported animals might provide evidence for vocal learning, whereas the changes in the local animals' call structure might show the effect of changes in the social situation of the animals. 